\title{
Spallation Neutron Source Second Target Station Integrated Systems Update
}

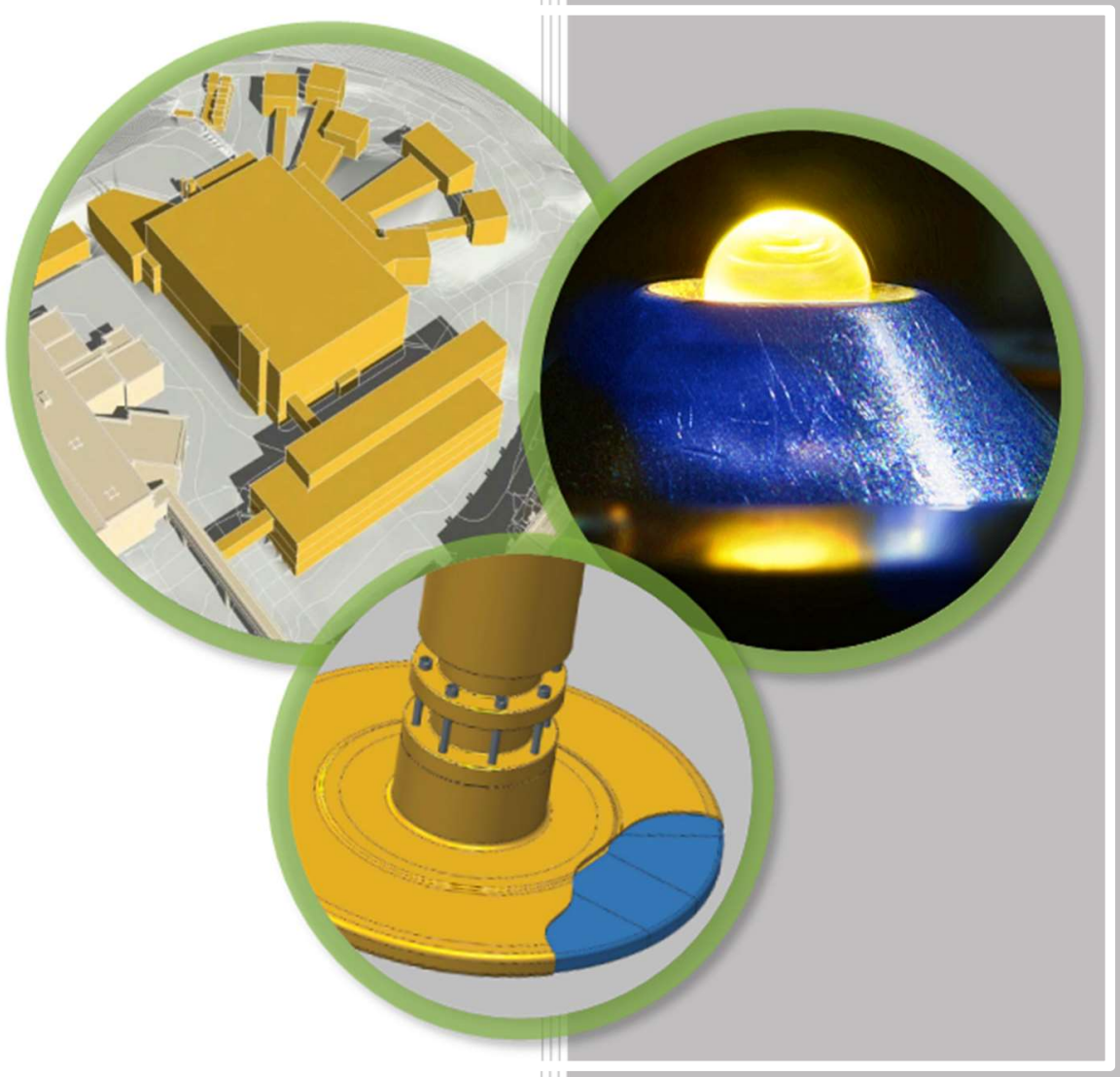

APPROVED FOR PUBLIC RELEASE. DISTRIBUTION IS UNLIMITED. 


\section{DOCUMENT AVAILABILITY}

Reports produced after January 1, 1996, are generally available free via US Department of Energy (DOE) SciTech Connect.

Website http://www.osti.gov/scitech/

Reports produced before January 1, 1996, may be purchased by members of the public from the following source:

National Technical Information Service
5285 Port Royal Road
Springfield, VA 22161
Telephone 703-605-6000 (1-800-553-6847)
TDD 703-487-4639
Fax 703-605-6900
E-mail info@ntis.gov
Website http://classic.ntis.gov/

Reports are available to DOE employees, DOE contractors, Energy Technology Data Exchange representatives, and International Nuclear Information System representatives from the following source:

Office of Scientific and Technical Information

PO Box 62

Oak Ridge, TN 37831

Telephone 865-576-8401

Fax 865-576-5728

E-mail reports@osti.gov

Website http://www.osti.gov/contact.html

This report was prepared as an account of work sponsored by an agency of the United States Government. Neither the United States Government nor any agency thereof, nor any of their employees, makes any warranty, express or implied, or assumes any legal liability or responsibility for the accuracy, completeness, or usefulness of any information, apparatus, product, or process disclosed, or represents that its use would not infringe privately owned rights. Reference herein to any specific commercial product, process, or service by trade name, trademark, manufacturer, or otherwise, does not necessarily constitute or imply its endorsement, recommendation, or favoring by the United States Government or any agency thereof. The views and opinions of authors expressed herein do not necessarily state or reflect those of the United States Government or any agency thereof. 
Neutron Sciences Directorate

\section{SPALLATION NEUTRON SOURCE SECOND TARGET STATION INTEGRATED SYSTEMS UPDATE}

Date Published: April 2017

Prepared by OAK RIDGE NATIONAL LABORATORY

Oak Ridge, TN 37831-6283

managed by

UT-BATTELLE, LLC

for the

US DEPARTMENT OF ENERGY

under contract DE-AC05-00OR22725 



\section{CONTRIBUTORS}

EDITORS: K. W. Herwig and M. J. Rennich

\section{CONTRIBUTORS}

R. Abudureyimu, J. Ankner, K. An, W. Blokland, T. Charlton, L. Coates, M. Dayton, R. Dean, E. Dominguez-Ontiveros, G. Ehlers, F. Gallmeier, V. Graves, W. Heller, J. Holmes, A. Huq, M. Lumsden, W. McHargue, T. McManamy, M. Plum, S. Rajic, I. Remec, L. Robertson, G. Sala, A. Stoica, S. Trotter and B. Winn 


\section{CONTENTS}

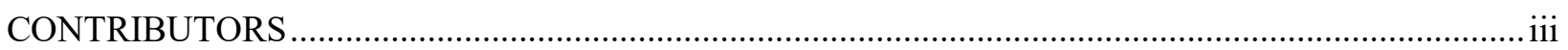

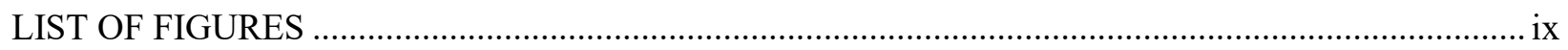

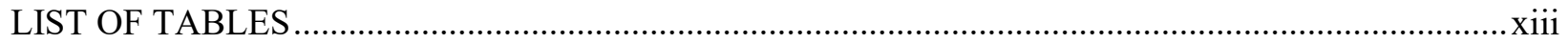

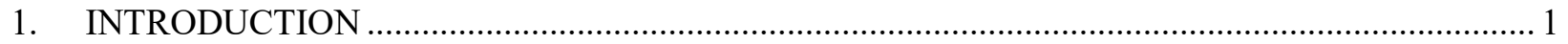

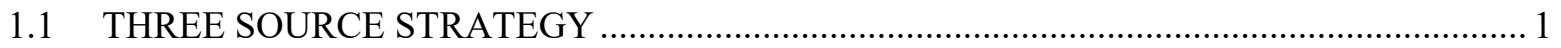

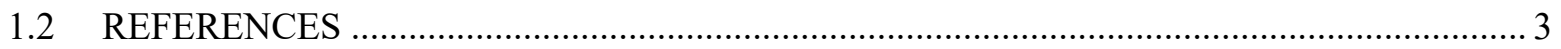

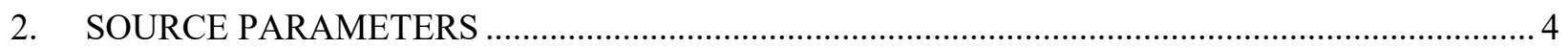

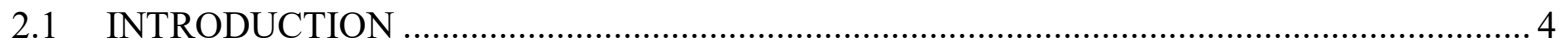

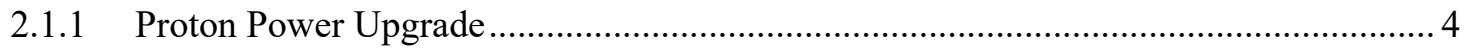

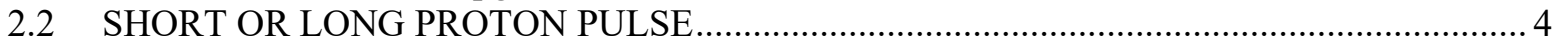

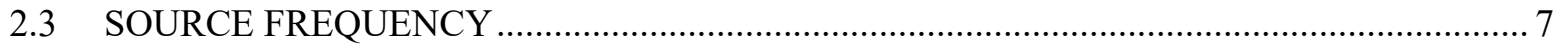

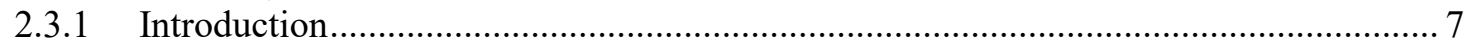

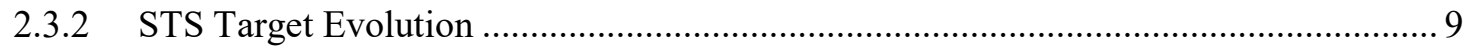

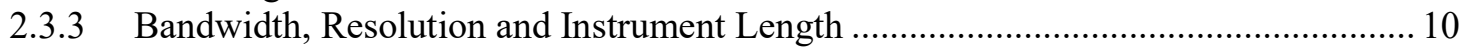

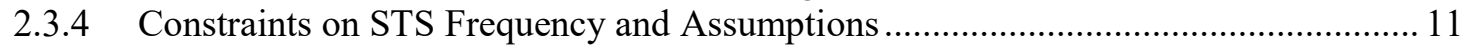

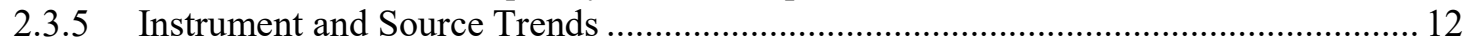

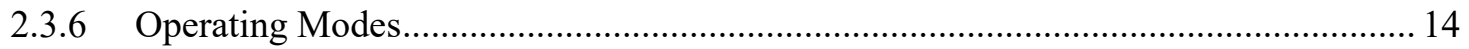

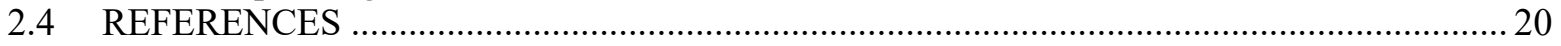

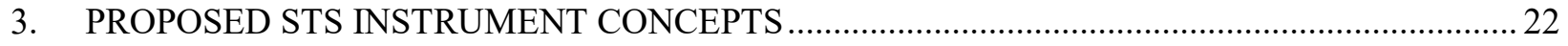

3.1 CHESS - CHOPPER SPECTROMETER FOR SMALL SAMPLES …............................... 22

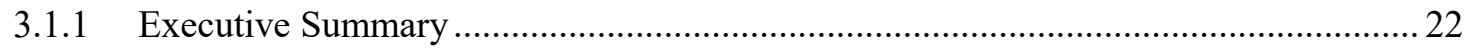

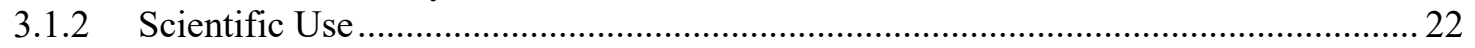

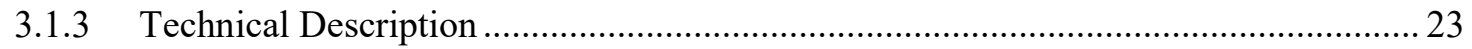

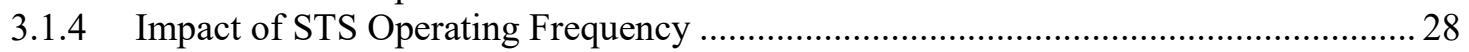

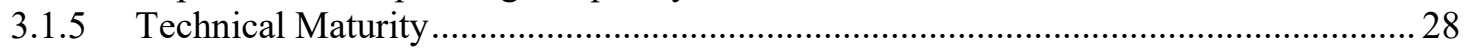

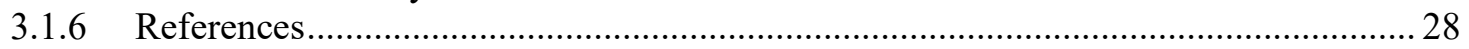

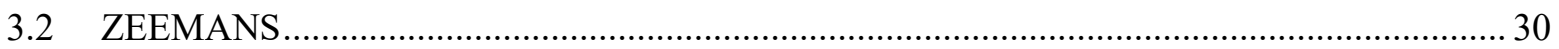

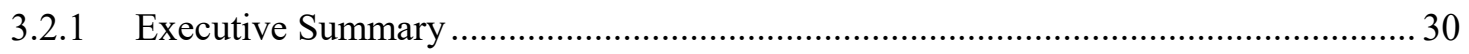

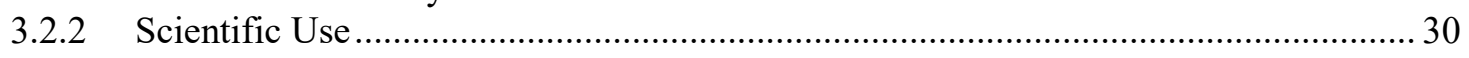

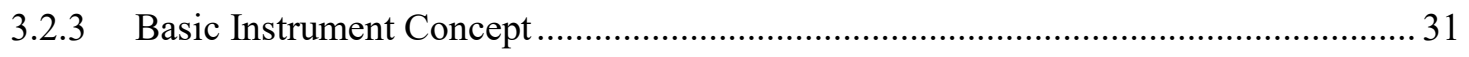

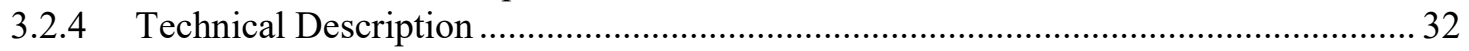

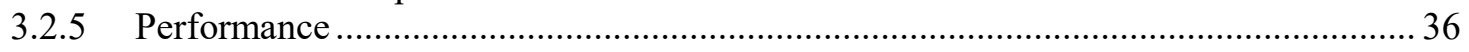

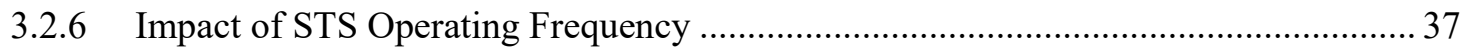

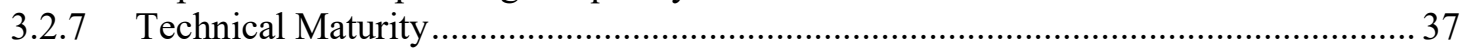

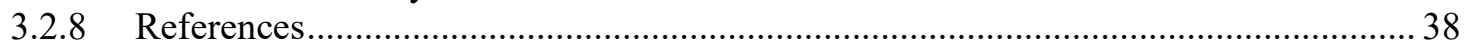

3.3 EWALD: AN EXTENDED WIDE ANGLE LAUE DIFFRACTOMETER FOR THE SECOND TARGET STATION OF THE SPALLATION NEUTRON SOURCE...................39

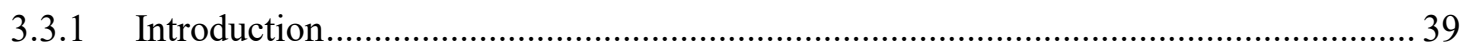

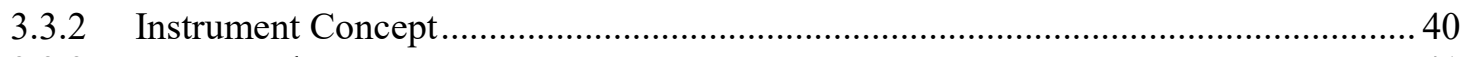

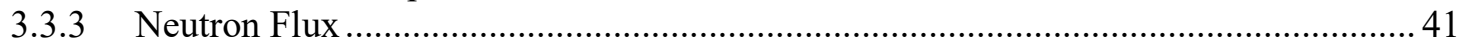

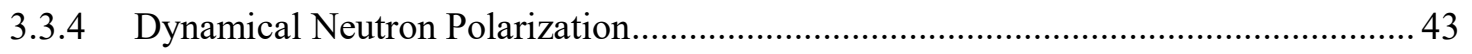

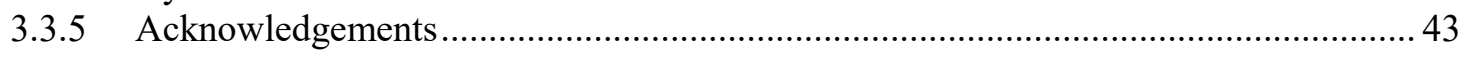

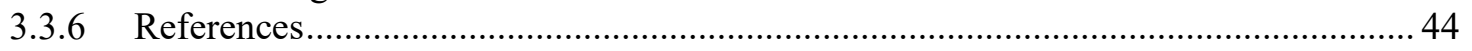

3.4 THE QUITE INTENSE KINETICS REFLECTOMETER (QIKR) .................................... 45

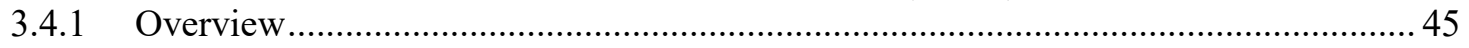

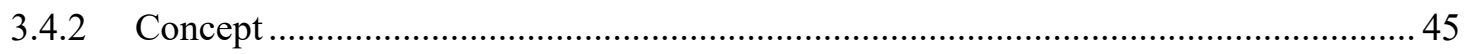

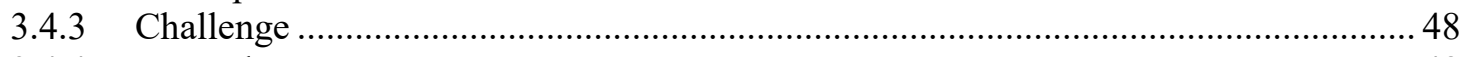

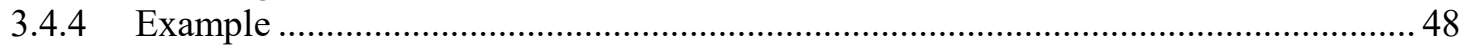


3.4.5 First-Day Experiment: Kinetics of EDA crosslinking in multilayer films of PMAA (Prof. E. Kharlampieva, U. Alabama, Birmingham) ....................................... 49

3.5 A SMALL-/WIDE-ANGLE NEUTRON SCATTERING INSTRUMENT AT STS ..............52

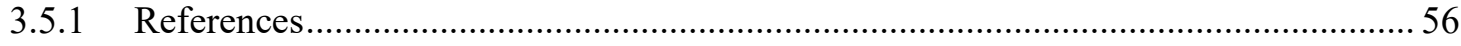

3.6 A SMALL-ANGLE NEUTRON SCATTERING INSTRUMENT AT STS .......................... 57

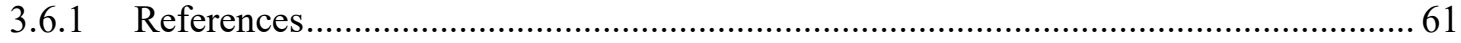

3.7 MENUS - MATERIALS ENGINEERING BY NEUTRON SCATTERING …...................62

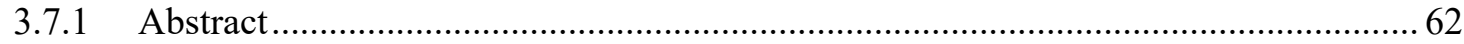

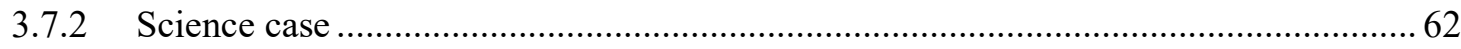

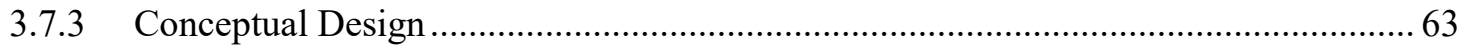

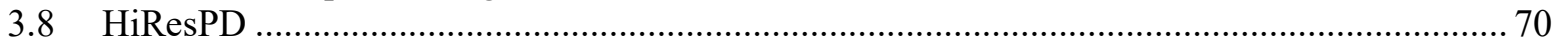

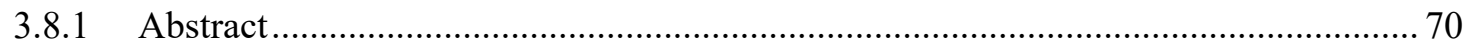

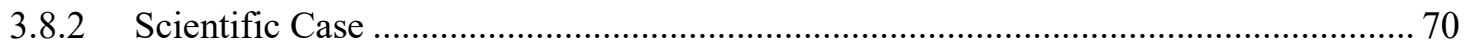

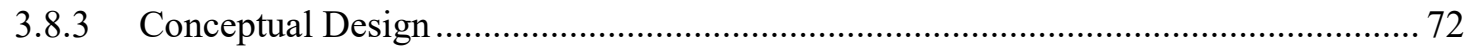

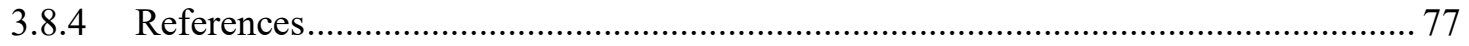

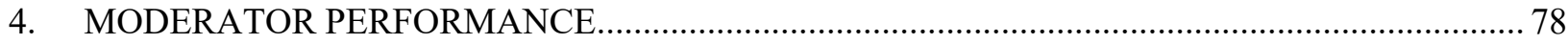

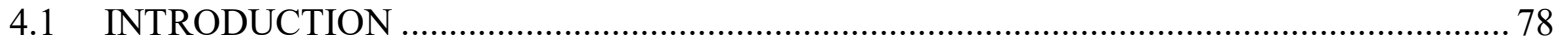

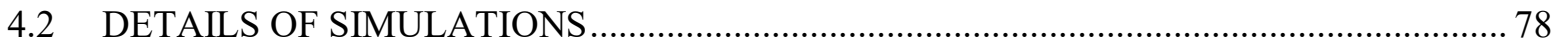

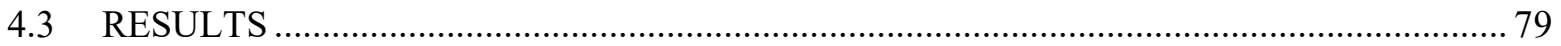

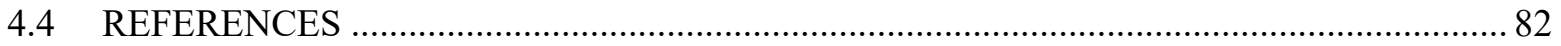

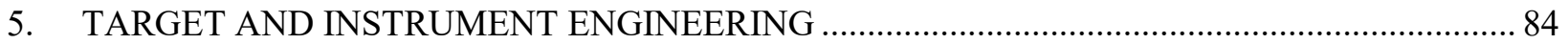

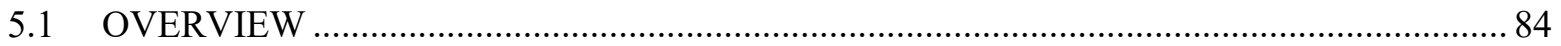

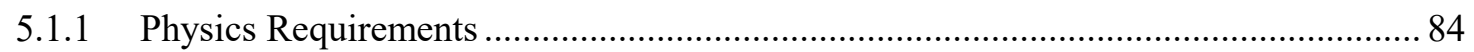

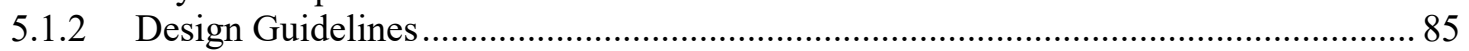

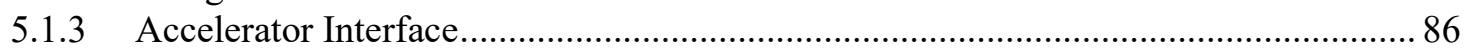

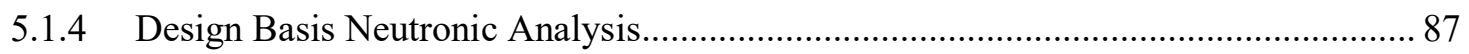

5.2 TARGET AND INSTRUMENT FACILITY CONFIGURATION ....................................... 91

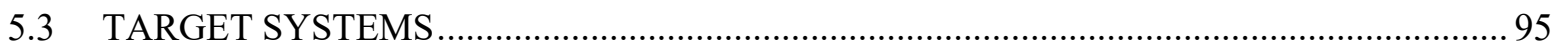

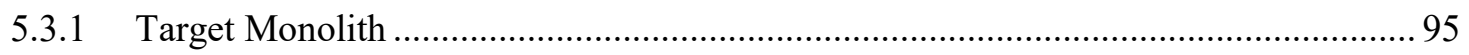

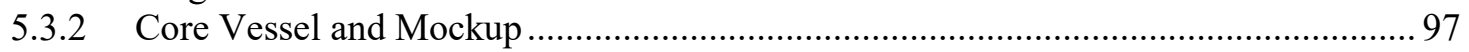

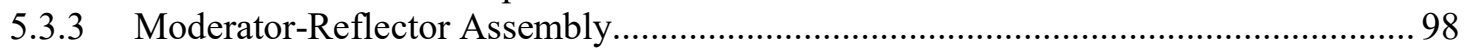

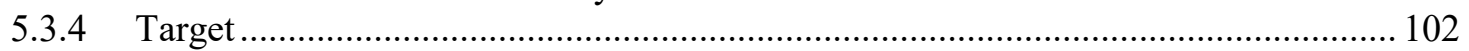

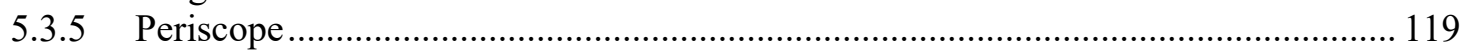

5.3.6 Proton Beam Window and Halo ...................................................................... 122

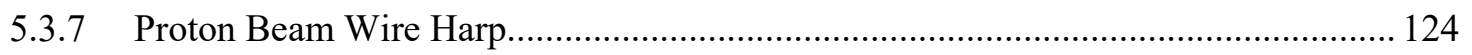

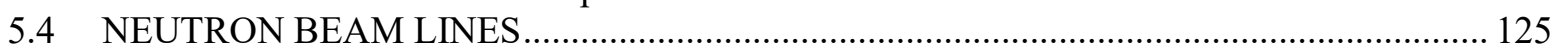

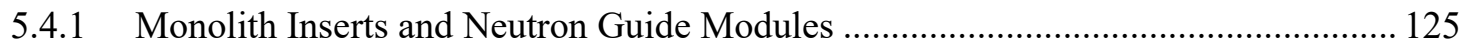

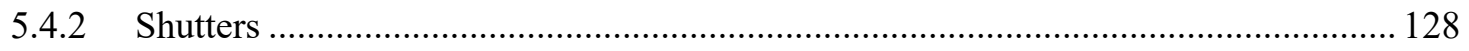

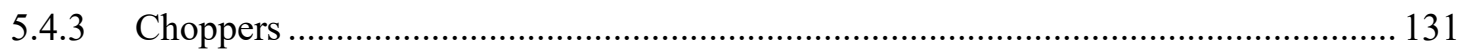

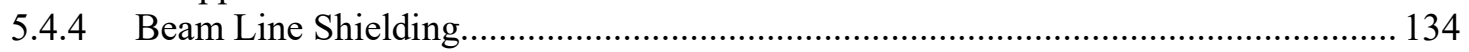

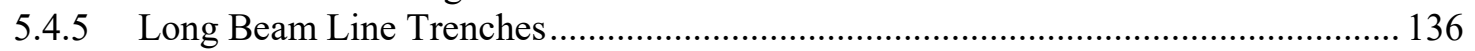

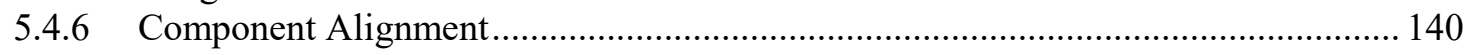

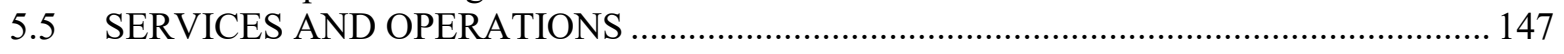

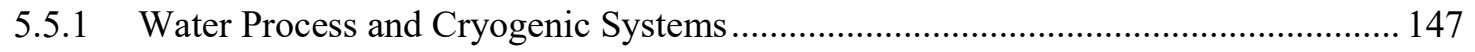

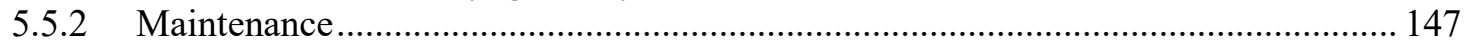

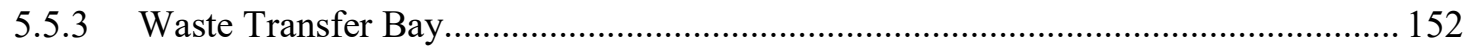

5.5.4 Activated Component Handling and Post Irradiation Examination........................... 153

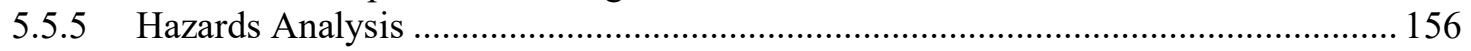

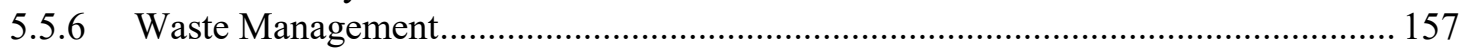

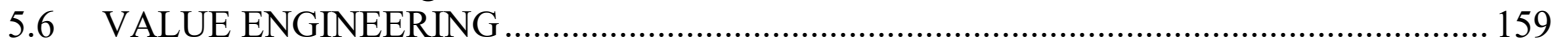

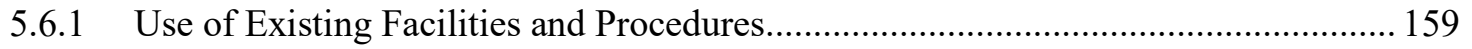




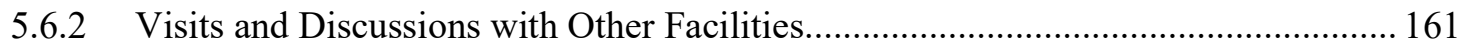

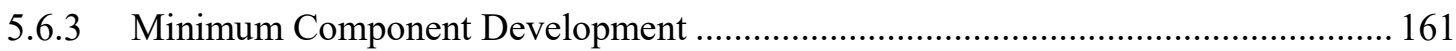

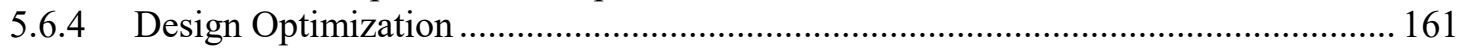

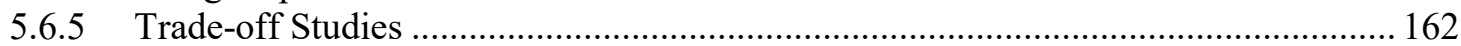

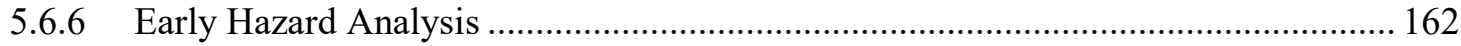

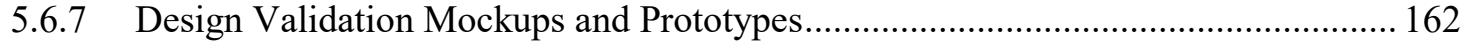

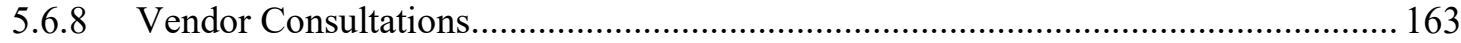

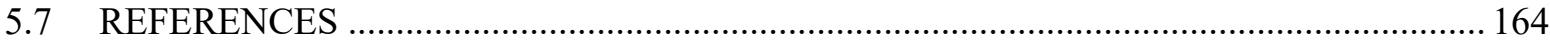

APPENDIX A. STS PLANNING INSTRUMENT SUITE .............................................................. 


\section{LIST OF FIGURES}

Figure 1.1. Comparison of the strengths of ORNL neutron sources....................................................... 2

Figure 1.2. Cold neutron pulse shapes multiplied by source frequency emitted by coupled moderators at existing, planned and proposed neutron sources................................................. 3

Figure 1.3. Thermal neutron pulse shapes multiplied by source frequency at existing, planned and

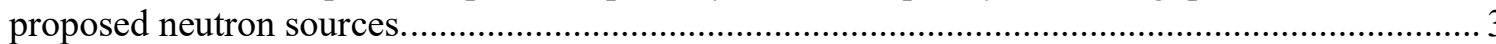

Figure 2.1. Coupled para-hydrogen moderator pulses optimized for long-pulse and short-pulse. $46.7 \mathrm{~kJ}$ proton pulses were used for each calculation...............................................................5

Figure 2.2. Comparison of performance for long and short proton pulse operation of STS cold,

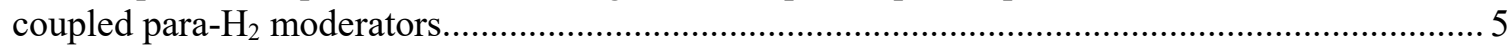

Figure 2.3. Wavelength resolution provided by the $1 \mathrm{msec}$ broad pulse of the STS-LP moderator as a function of neutron wavelength at the indicated instrument lengths.

Figure 2.4. Time averaged neutron brightness when frame suppression is used to effectively broaden the neutron wavelength bandwidth.

Figure 2.5. Graphical illustration of the operating rhythms for STS and FTS at relevant STS operating frequencies of $10 \mathrm{~Hz}, 15 \mathrm{~Hz}$, and $20 \mathrm{~Hz}$ as indicated in vertical sections 2-4 above.

Figure 2.6. Neutron flux multiplied by $\lambda 4$ to represent the value of longer wavelength neutrons to a reflectometry measurement.

Figure 3.1. Conceptual engineering layout for CHESS that shows how the instrument will fit in its environment in the facility.

Figure 3.2. Left: Two ballistic sections of neutron guide transport the beam from the source

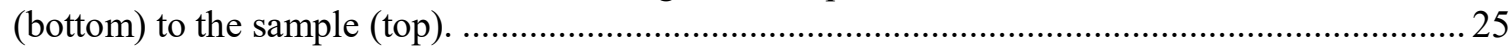

Figure 3.3. Preliminary neutron transport calculation. Beam on sample is shown................................. 26

Figure 3.4. Concept of the ZEEMANS instrument......................................................................... 31

Figure 3.5. Test stack of pancakes coils employing no-insulation ReBCO composite conductor [6]. 32

Figure 3.6. Isometric rendering of the magnet design being evaluated, cut at beam elevation, showing the wedge-support geometry of the magnet and access for incident and scattered beams. 33

Figure 3.7. Timing diagram for $15 \mathrm{~Hz}$ operation with a source to sample distance of $45 \mathrm{~m}$.....................34

Figure 3.8. Moderator brightness (n/sr/pulse/ $\AA$ ) for the $5 \mathrm{~cm} \mathrm{x} 5 \mathrm{~cm}$ coupled cold moderator for STS. 36

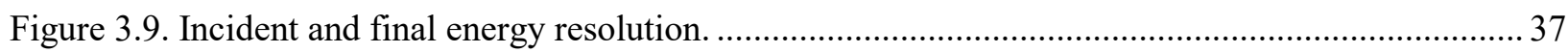

Figure 3.10: Laue diffraction image from a single SNS Anger camera............................................... 40

Figure 3.11. A schematic overview of the neutron optics on Ewald showing the location of the moderator, primary slit, Kirkpatrick-Baez (KB) neutron supermirrors, sample position, detector array and the diffraction pattern from a single detector module.

Figure 3.12. Monte Carlo simulated diffraction patterns from a protein crystal $0.01 \mathrm{~mm}^{3}$ in volume on the EWALD instrument.

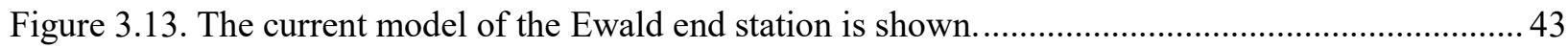

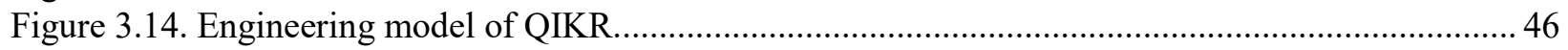

Figure 3.15. Measured output of the SNS Liquids Reflectometer tapered guide. ................................... 47

Figure 3.16. QIKR shutter concept and table of beam heights vs distance from the moderator................ 48

Figure 3.17. Parameters for alternating layers of deuterated and conventional polystyrene. .................... 48

Figure 3.18. Simulated QIKR data for polymer diffusion study .............................................................. 49

Figure 3.19. Ionization of PMAA in the crosslinking of the multilayer film ........................................ 50

Figure 3.20. Reflectivity and SLD plots of spin-assisted PMAA multilayers H-bonded with PVPON (top) and after crosslinking/release of PVPON(bottom). 
Figure 3.21. Simulated QIKR data for determining film thickness during EDA crosslinking .................50

Figure 3.22. Schematic of the layout envisioned for the components of the SWANS instrument.............53

Figure 3.23. (left) Peak brightness of the two possible moderators that the SWANS instrument

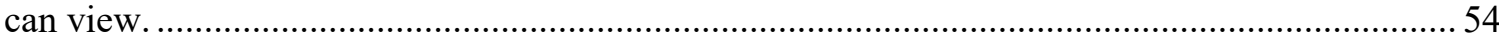

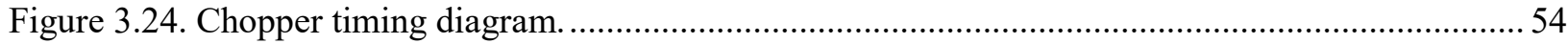

Figure 3.25. Schematic showing the angular ranges covered by the SWANS detector array. .................. 55

Figure 3.26. (top) Estimated flux on sample for the SWANS as a function of wavelength.

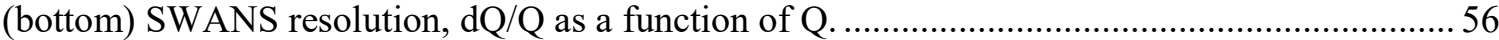

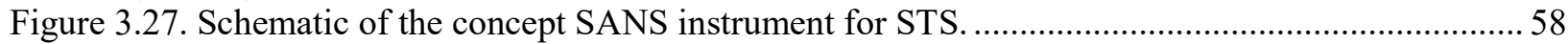

Figure 3.28. Brightness of the moderators that a SANS instrument can view...................................... 58

Figure 3.29. Chopper timing diagram for (top) low-Q detector at $10 \mathrm{~m}, 15 \mathrm{~Hz}$ operation and

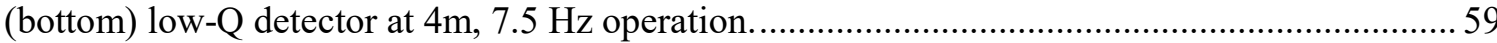

Figure 3.30. Time-averaged flux on sample for the SANS operating at $15 \mathrm{~Hz}$ for the three

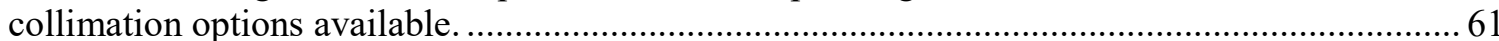

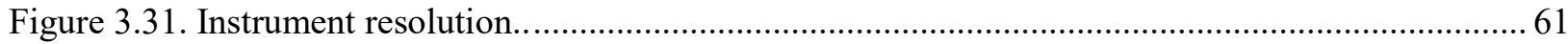

Figure 3.32. The MENUS optics design, a) the schematic view, b) the optics details in horizontal

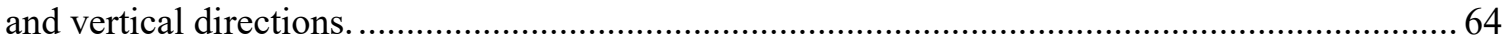

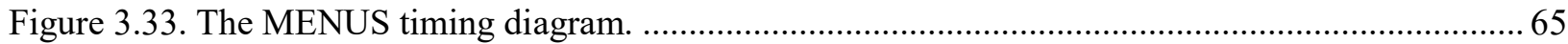

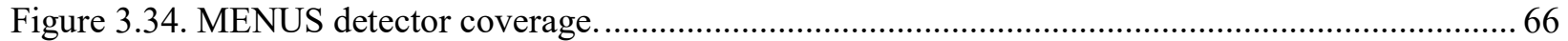

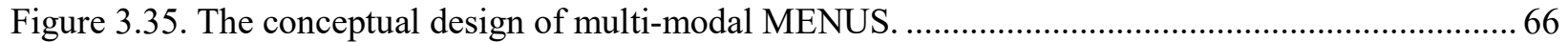

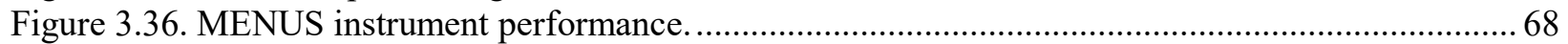

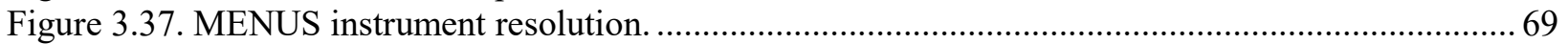

Figure 3.38. Magnified region of the refinement of synchrotron x-ray diffraction data after convergence for the sample with composition of $\mathrm{Li} 6.5 \mathrm{La} 3 \mathrm{Zr} 1.5 \mathrm{Ta} 0.5 \mathrm{O} 12$ highlighting

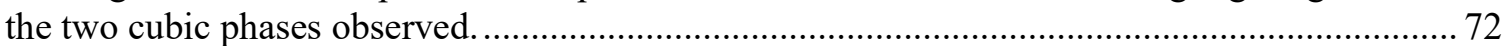

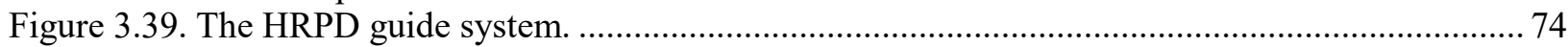

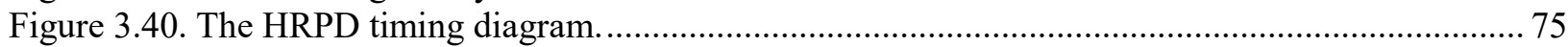

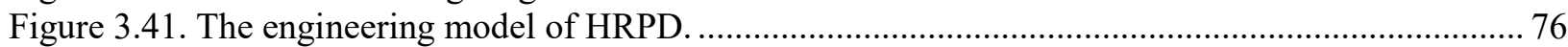

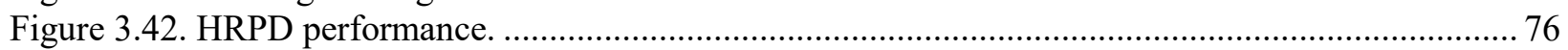

Figure 4.1. MCNPX model of optimized STS target/moderator/reflector arrangement........................... 79

Figure 4.2. Comparison of cold coupled moderator performance metrics, of time-averaged and peak brightness and FWHM pulse width, of STS and FTS moderators vs energy(left) and

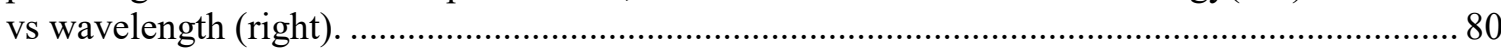

Figure 4.3. Comparison of cold decoupled hydrogen and ambient temperature water moderator performance metrics, of time-averaged and peak brightness and FWHM pulse width, of

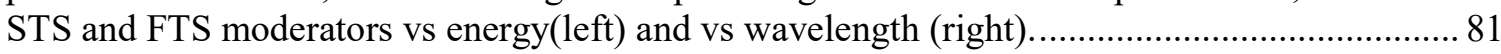

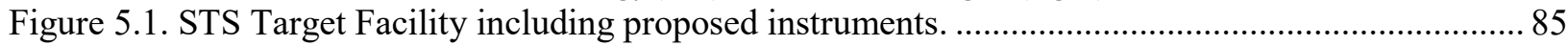

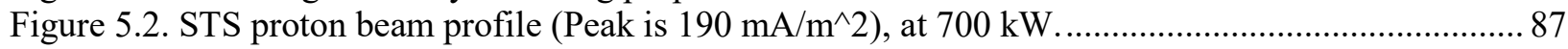

Figure 5.3. Neutronics model obtained by automatic conversion of the ProE Creo mechanical

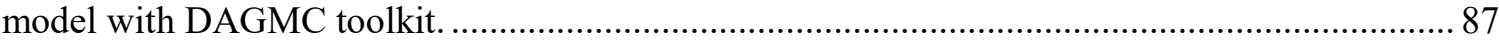

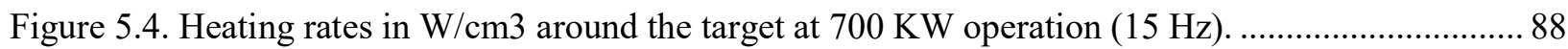

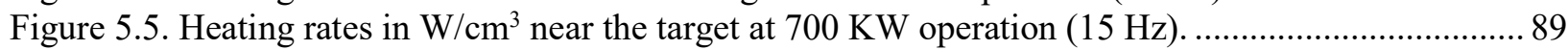

Figure 5.6. Total $(n+p)$ dpa per year in stainless steel; 5000 hours per year of beam-on-operation

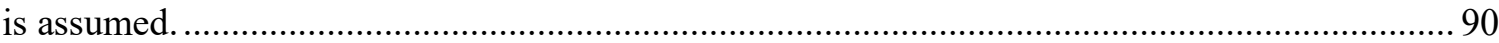

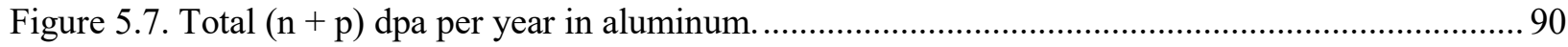

Figure 5.8. Target building horizontal section at proton beam level. .................................................. 91

Figure 5.9. Target building vertical section view along proton beam line............................................ 92

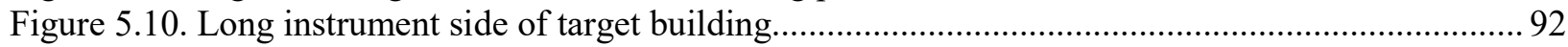

Figure 5.11. Target building section view showing neutron beam line bunkers. .................................. 93

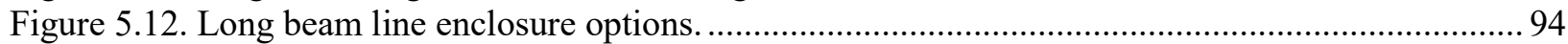

Figure 5.13. Long beam line trenches viewed from just outside the outer bunker wall. ........................ 95 
Figure 5.14. Section view along proton beam line of target station monolith and core vessel................. 96

Figure 5.15. Section view of core vessel showing location of primary components................................96

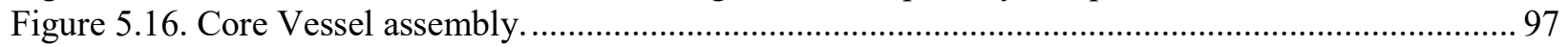

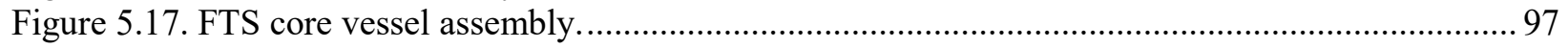

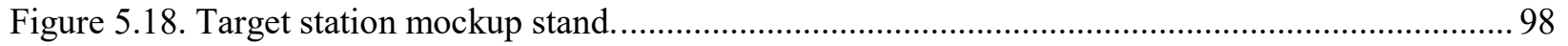

Figure 5.19. The relationship between the target and the moderators in the beam-on operating

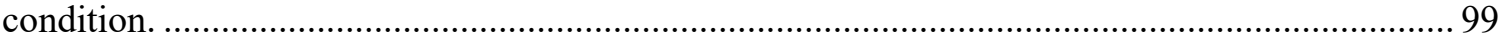

Figure 5.20. Vertical section view of target station core showing moderator-reflector assembly........... 100

Figure 5.21. CFD predicted temperature distribution in the lower coupled moderator with a liquid

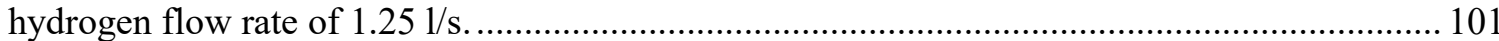

Figure 5.22. Exploded view of the moderator-reflector plug showing modular construction................. 102

Figure 5.23. Maximum target temperature and decay heat versus time after a loss of coolant accident with beam trip by target protection system or seismic motions................................... 103

Figure 5.24. Vertical section view of target station core with rotating target assembly highlighted........ 104

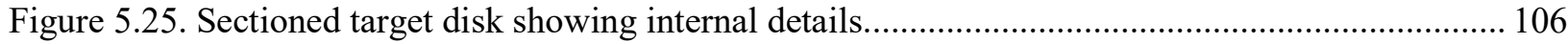

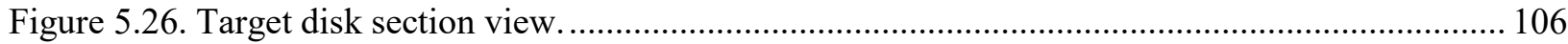

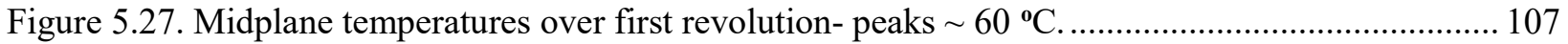

Figure 5.28. 3 Block model global peak temperature versus time for first 6 revolutions and 4

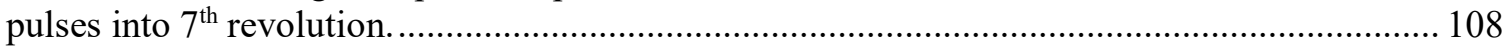

Figure 5.29. Temperatures after 6 revolutions plus 4 pulses into $7^{\text {th }}$ revolution................................. 108

Figure 5.30. Tantalum surface Von Mises Stress - Peak 63 MPa.................................................. 109

Figure 5.31. Tungsten surface Von Mises stress with clad hidden on two blocks................................... 109

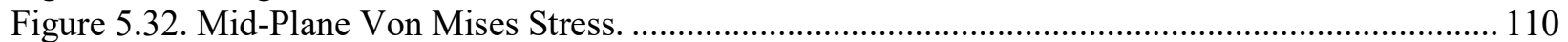

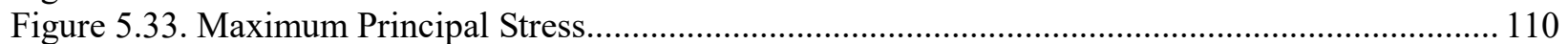

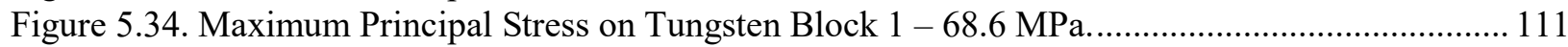

Figure 5.35. Tungsten plate thermal expansion after seven revolutions.............................................. 111

Figure 5.36. Calculated operating stress and deflection plots with $0.35 \mathrm{MPa}$ internal water

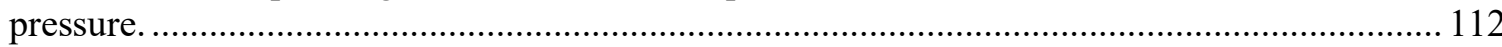

Figure 5.37. Comparison of peak plate temperatures in STS stationary target vs STS rotating

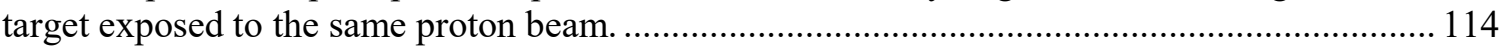

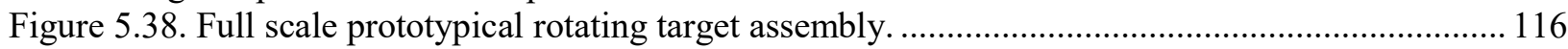

Figure 5.39. Target disk decay heat after 20 years of $15 \mathrm{~Hz}$ beam operation....................................... 117

Figure 5.40. Target and Monolith Heating7 analysis model for loss of cooling with decay heat............ 118

Figure 5.41. Peak temperatures at 11 hours and after 30 days..................................................... 118

Figure 5.42. Peak temperatures around the target disk after 11 hours................................................ 119

Figure 5.43. Section target monolith along periscope sightline................................................. 120

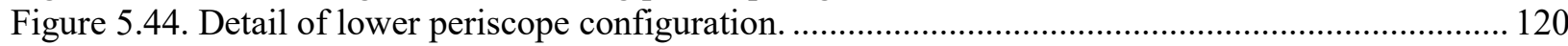

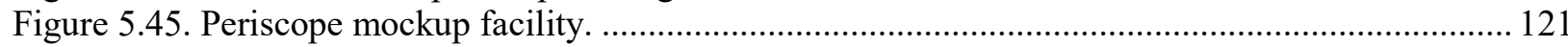

Figure 5.46. Mockup periscope target image showing resolution and depth of field............................ 121

Figure 5.47. Temperature distribution and deflection in first periscope mirror during beam-on

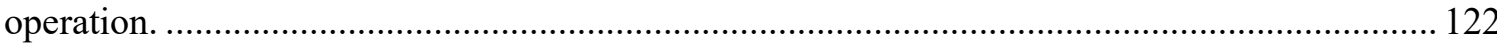

Figure 5.48. Proton beam heating and helium in aluminum proton beam window based on a 10

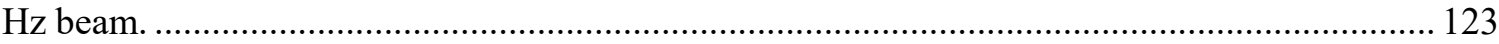

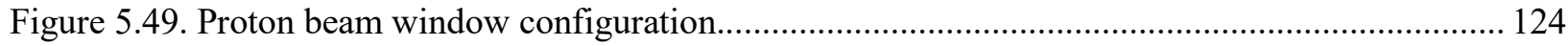

Figure 5.50. Current SNS proton beam Harp module...................................................................... 124

Figure 5.51. Typical neutron beam line monolith insert with guide module....................................... 126

Figure 5.52. Monolith inserts viewing the two lower moderators. .................................................. 127

Figure 5.53. Guide module being inserted into a monolith insert................................................... 127

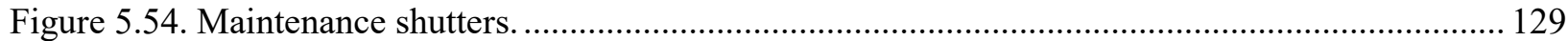

Figure 5.55. STS operating shutter concepts for low- and high-energy neutrons.................................. 130

Figure 5.56. Operating shutter within the outer bunker wall........................................................... 130 
Figure 5.57. Comparison of $10 \mathrm{~Hz}$ and $15 \mathrm{~Hz}$ chopper diameters.

Figure 5.58. STS bunker views showing impact of source frequency on chopper size.

Figure 5.59. Close spacing of $\mathrm{T}_{0}$ choppers within the STS bunker.

Figure 5.60. STS $10 \mathrm{~Hz}$ chopper library.

Figure 5.61. Estimated shielding thicknesses along the STS beam lines.

Figure 5.62. Beam line shielding extent.

Figure 5.63. Long instruments in the STS.

Figure 5.64. FTS BASIS beam line.

Figure 5.65. Typical trench cross section.

Figure 5.66. Personnel access of beam line components within a trench........................................... 139

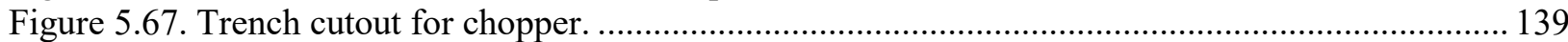

Figure 5.68. Geometric relationship between target and moderators (proton beam enters page)............ 140

Figure 5.69. Moderator-reflector assembly installation interface.................................................. 141

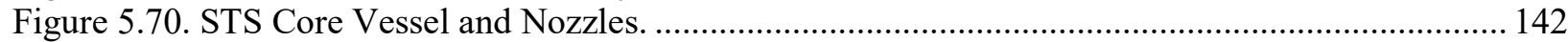

Figure 5.71. External alignment features of monolith inserts....................................................... 143

Figure 5.72. Internal kinematic alignment features of the monolith inserts. ....................................... 143

Figure 5.73. STS MENUS focusing mirror assembly on remotely actuated precision movers............... 145

Figure 5.74. Final focusing mirror within STS EWALD instrument concept. .................................... 145

Figure 5.75. Images from SLAC-PUB-95-6132 showing the operating principle of 3- and 5-DOF

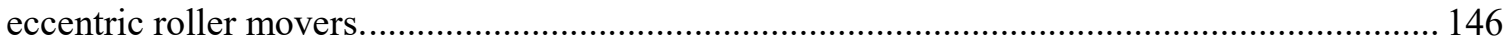

Figure 5.76. Examples of magnet mover implementations at SLS, SLAC, and KEK........................... 146

Figure 5.77. PBW window removal operations as proposed for STS and as implemented at FTS......... 149

Figure 5.78. The moderator/reflector plug moved horizontally to clear target disk to allow vertical

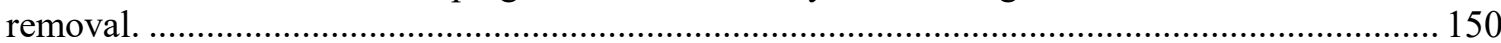

Figure 5.79. Target handling container positioned on core vessel during removal operation................. 151

Figure 5.80. Monolith insert handling machine located in the guide bunker...................................... 152

Figure 5.81. Target handling container positioned in transfer bay.................................................. 152

Figure 5.82. Comparison of FTS and STS facility space utilization requirements............................... 160 


\section{LIST OF TABLES}

Table 2.1. Advantages and disadvantages associated with the indicated STS operating frequency............ 8

Table 2.2. Operation of FTS and STS in pulse-stealing mode. It is assumed that regardless of

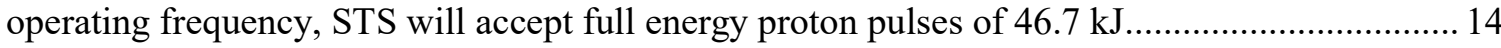

Table 2.3. FTS instrument operation in frame suppression mode ...................................................... 15

Table 2.4. Operating modes of FTS instruments when STS operates at the indicated frequencies. The last column lists the part of the proton power directed to FTS that is used in FTS frame suppression mode.

Table 2.5. Instruments identified for STS beam lines with identified lengths and corresponding

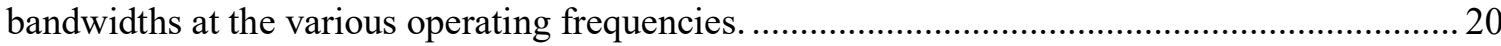

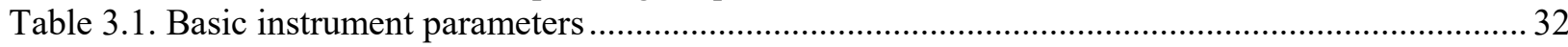

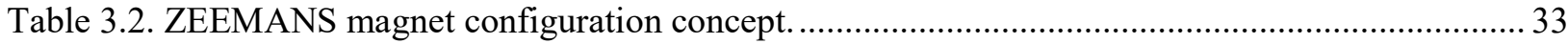

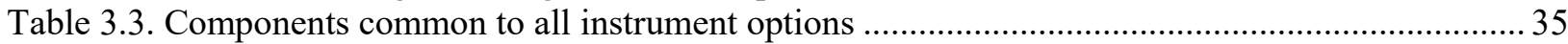

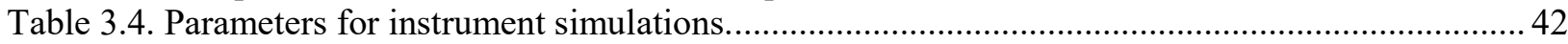

Table 3.5. Bandwidth of neutron wavelengths and associated Q-ranges for the detector banks on

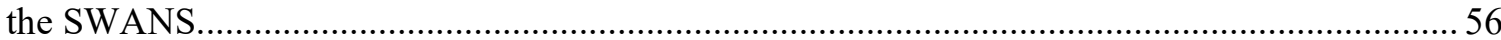

Table 3.6. Bandwidth of neutrons, associated Q-ranges and integrated flux on sample........................... 60

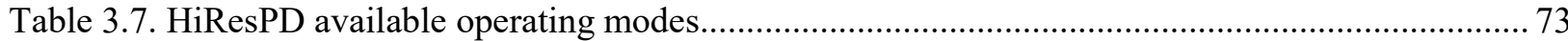

Table 4.1. Gains over the present FTS at 1.4 MW and STS moderators with results as high as 6 and 30 for the smallest sized coupled moderator in time-integrated brightness, and peak

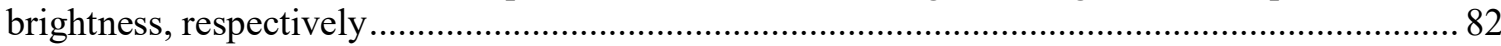

Table 5.1. Accelerator characteristics of various neutron scattering facilities...................................... 86

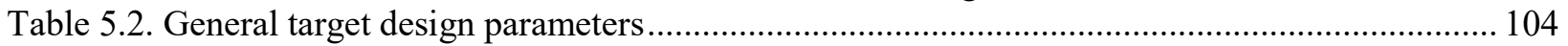

Table 5.3. Summary of various tantalum clad tungsten target thermos-mechanical factors.................... 112

Table 5.4. FTS shutter use during beam-on operations between Jan 21 and Apr 21, 2015 ( 13

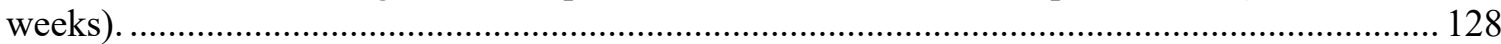

Table 5.5. Estimated activated component life-times at full power, $5000 \mathrm{hr} / \mathrm{yr}$ operation...................... 148 


\section{INTRODUCTION}

The Spallation Neutron Source (SNS) was designed from the beginning to accommodate both an accelerator upgrade to increase the proton power and a second target station (STS). Four workshops were organized in 2013 and 2014 to identify key science areas and challenges where neutrons will play a vital role [1-4]. Participants concluded that the addition of STS to the existing ORNL neutron sources was needed to complement the strengths of High Flux Isotope Reactor (HFIR) and the SNS first target station (FTS). To address the capability gaps identified in the workshops, a study was undertaken to identify instrument concepts that could provide the required new science capabilities. The study outlined 22 instrument concepts and presented an initial science case for STS [5]. These instrument concepts formed the basis of a planning suite of instruments whose requirements determined an initial site layout and moderator selection. An STS Technical Design Report (TDR) documented the STS concept based on those choices [6]. Since issue of the TDR, the STS concept has significantly matured as described in this document.

This chapter describes the role of STS within the ORNL 3-source strategy. Chapter 2 describes the reasoning underlying the choices of operating the STS as a short-proton pulse source at $15 \mathrm{~Hz}$. Chapter 3 describes concepts for 7 of the 12 high-priority neutron scattering instruments identified in an October 2015 user workshop [7] and includes initial evaluation of the performance of a general-purpose SANS instrument. Chapter 4 discusses the optimization and performance of the current STS moderator suite. Chapter 5 presents the current state of Target and Instrument Engineering, including the relatively recent replacement of the TDR stationary, solid target with a rotating target.

\subsection{THREE SOURCE STRATEGY}

Figure 1.1 compares the strengths of the ORNL facilities. STS produces the highest peak brightness of cold neutrons as shown in Figure 1.1(a) (and illustrated in Figure 1.2) of any current or proposed neutron sources. Neutron scattering instruments whose performance scales with peak brightness of cold neutrons will be optimally located at STS. HFIR produces the highest time-averaged cold neutron brightness of any of the ORNL sources as shown in Figure 1.1(b), is comparable to the world's best reactor sources and is within a factor of 3 of the predicted performance of the European Spallation Source (ESS) currently under construction in Lund, Sweden. It is best matched to neutron scattering techniques that optimally use relatively low wavelength resolution like a traditional SANS or to examine a small volume of reciprocal space with high efficiency like a triple-axis spectrometer enabling, for example, rapid parametric studies. Neutron scattering instruments that are optimized for highest time-averaged brightness will be optimally located at HFIR. FTS is optimized for highest neutron wavelength resolution as illustrated in Figure 1.1(c) with its emphasis on poisoned, decoupled moderators that produce very sharp in time neutron pulses while its $60 \mathrm{~Hz}$ operating frequency is optimized for narrow-bandwidth instruments. Note the graphs in Figure 1.1 are generated for FTS at its full rated power of $2 \mathrm{MW}$ which will be enabled by the proton power upgrade project that also provides the intense proton pulses powering the STS. The strength of the first target station is further illustrated in Figure 1.3 that shows the sharp peaks generated by a poisoned water moderator multiplied by the source frequency in comparison with the corresponding ESS pulse generated by its water moderator and the J-PARC poisoned moderator pulse as predicted for full power operation (ESS at $5 \mathrm{MW}$, and J-PARC at $1 \mathrm{MW}$ ). (J-PARC is the MW-class pulsed spallation source located at Japan-Proton Accelerator Research Complex and its poisoned moderator is cold hydrogen.) STS ideally complements the existing ORNL sources by producing broader cold neutron pulses and wide dynamic range with its emphasis on coupled, cold moderators and low source frequency as illustrated in Figure 1.3. The peak shapes were generated at source frequencies of $25 \mathrm{~Hz}, 14 \mathrm{~Hz}$ and 15 $\mathrm{Hz}$ for J-PARC at $1 \mathrm{MW}$, ESS at $5 \mathrm{MW}$, and STS at $0.7 \mathrm{MW}$ respectively. With the high time-averaged brightness of HFIR, in combination, these three neutron sources will provide the U.S. research 
community with access to a sweeping set of tools required to address the challenging science questions of the next decades. STS is essential to fill the gap between FTS and HFIR and provide U.S. researchers access to the science capabilities the fourth-generation ESS neutron source will provide for European researchers.
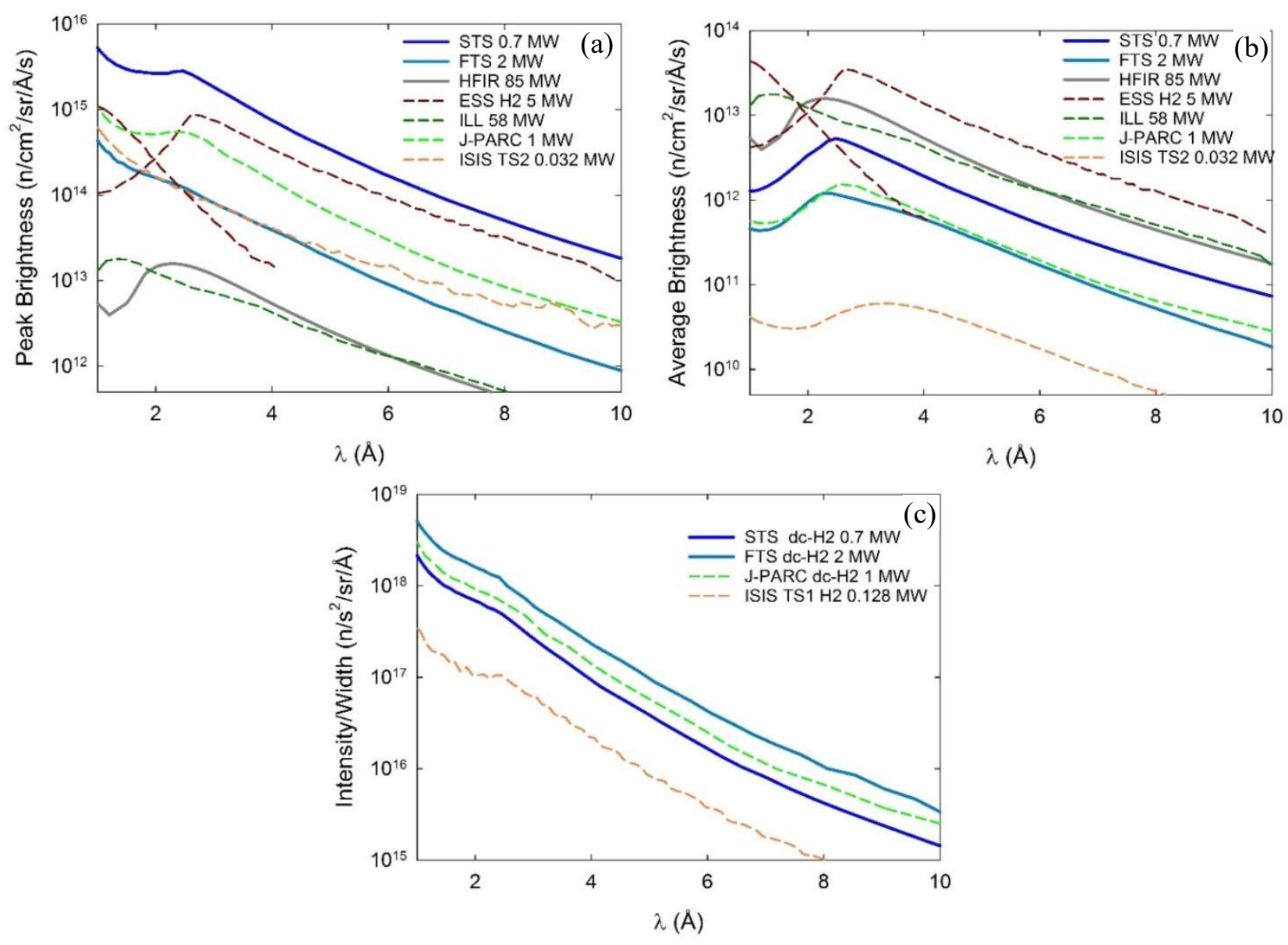

Figure 1.1. Comparison of the strengths of ORNL neutron sources. (a) is the peak brightness of cold neutrons that STS is optimized to produce, (b) is the time-averaged cold brightness that is the strength of HFIR, and (c) is the moderator intensity divided by moderator pulse width which is a reasonable metric for high wavelength resolution instruments and is the forte of FTS. 


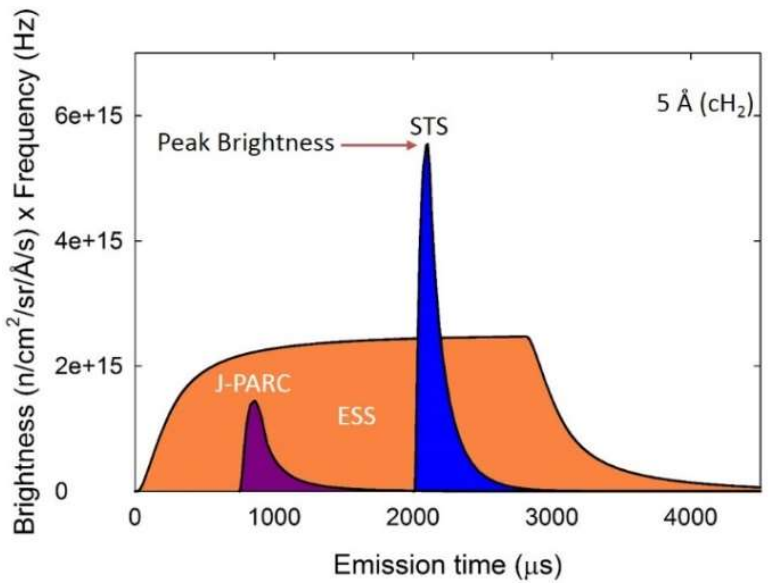

Figure 1.2. Cold neutron pulse shapes multiplied by source frequency emitted by coupled moderators at existing, planned and proposed neutron sources.

Curves are offset horizontally for clarity.

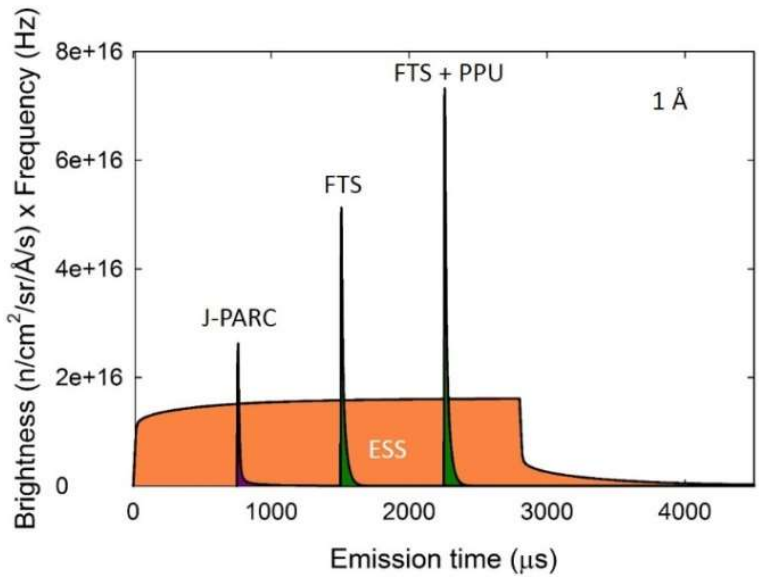

Figure 1.3. Thermal neutron pulse shapes multiplied by source frequency at existing, planned and proposed neutron sources. Curves are offset horizontally for clarity. FTS is the SNS first target station pulse from a water moderator at proton beam power of 1.4 MW (FTS + PPU is the same moderator at $2 \mathrm{MW}$ ).

\subsection{REFERENCES}

[1] Quantum Condensed Matter Workshop Report, December 5-6, 2013, Lawrence Berkeley National Laboratory, Bob Birgeneau, University of California-Berkeley.

[2] Grand Challenges in Soft Matter Workshop Report, May 17-18, 2014, University of CaliforniaSanta Barbara (UCSB), Fyl Pincus (UCSB) and Matt Tirrell, University of Chicago.

[3] Grand Challenges in Biological Neutron Scattering Report, January 17-18, 2014, University of California-San Diego (UCSD), Susan Taylor, UCSD, and Heidi Hamm, Vanderbilt University.

[4] Frontiers in Materials Discover, Characterization, and Application Workshop Report, August 2-3, 2014, Schaumburg, Illinois, George Crabtree, Argonne National Laboratory, and John Parise, University of Stony Brook.

[5] Instruments for Emerging Science: A Science Case for the Second Target Station, ed. K. W. Herwig and D. A. Tennant, Neutron Sciences Directorate, ORNL, 2014 (unpublished).

[6] Technical Design Report Second Target Station, ORNL/TM-2015/24, January 2015.

[7] Second Target Station Workshop, Co-chairs: M. R. Eskildsen (University of Notre Dame) and B. Khaykovich (Massachusetts Institute of Technology), https://public.ornl.gov/conferences/neutrons/STS2015/index.shtml. 


\section{SOURCE PARAMETERS}

\section{$2.1 \quad$ INTRODUCTION}

The Proton Power Upgrade (PPU) project will upgrade the SNS accelerator with a goal to raise the power of the proton beam delivered to the SNS First Target Station (FTS) to its maximum design limit of 2 MW and provide the intense proton pulses required for a Second Target Station (STS). The SNS accelerator will be able to deliver up to $46.7 \mathrm{~kJ}$ proton pulses to either FTS (within its 2 MW limit) or STS in any sequence consistent with $60 \mathrm{~Hz}$ accelerator operation. FTS was designed from the beginning to be a shortproton pulse spallation source with the SNS accelerator complex including an accumulator ring to produce a $<1 \mu \mathrm{sec}$ long proton pulse. Section 2.2 discusses the trade-offs between operating STS in a similar short-proton pulse mode or directing the linac output directly to the STS target without compression in the accumulator ring in long-proton pulse mode. The conclusion is that STS should also take advantage of the existing accumulator ring to operate as a short-proton pulse neutron source, and this is a key element of the STS strategy to produce the highest peak brightness neutron pulses. The recent adoption of a rotating target concept has raised the possibility to increase the power of the proton beam delivered to the STS target. This implies operating STS at a higher frequency, as the concept has always adopted using the full $46.7 \mathrm{~kJ}$ pulses the upgraded accelerator can produce. Section 2.3 discusses the trade-offs in increasing the STS operating frequency, concluding that $15 \mathrm{~Hz}$ is the optimal choice.

\subsubsection{Proton Power Upgrade}

The PPU project will upgrade the SNS accelerator capability to provide $46.7 \mathrm{~kJ} /$ pulse at $60 \mathrm{~Hz}$ (or 2.8 MW) [1]. This will be done by increasing the energy per accelerated proton to $1.3 \mathrm{GeV}$ and the average linac current to $38 \mathrm{~mA}$. The energy delivered per pulse is the product of the proton energy and the number of protons per pulse. Capacity to accelerate protons to higher energy or accelerating more protons per pulse (either with increased linac beam current or linac duty factor) is not in the PPU scope. Further increase in energy per proton is not possible, because the linac-accelerated $\mathrm{H}$ - ions will be stripped by the bending magnetic field in the HEBT transport line above $1.3 \mathrm{GeV}$ and not transported further. Increased linac duty factor beyond present levels is not possible because of cooling limitations in the lower energy copper linac structures, and reliability of the RF power sources. Increased average linac beam current beyond PPU levels requires: 1) higher RF source and supporting high voltage power supply levels beyond those provided by PPU, and 2) higher RF coupler power levels in the Superconducting RF cavities, beyond the $700 \mathrm{~kW}$ PPU design requirement.

PPU will not change the duty factor of the accelerator; it will continue to operate at $60 \mathrm{~Hz} \times 1 \mathrm{~ms}$. As stated, the maximum energy available in a single pulse will be $46.7 \mathrm{~kJ}$, but the accelerator will be able to reduce the number of protons in each pulse below the maximum corresponding to $46.7 \mathrm{~kJ} / \mathrm{pulse}$, and thus the energy delivered per pulse to FTS and STS can be different. Pulses can be directed to STS or FTS at rates as desired within the accelerator repetition rate of $60 \mathrm{~Hz}$. The two sources can be operated independently of one another with one source "off" and the other "on" (e.g. in the case of equipment failure at one source or possibly to stagger maintenance periods).

\subsection{SHORT OR LONG PROTON PULSE}

The SNS accelerator complex includes an accumulator ring to stack the mini pulses accelerated through the linac on top of each other intensifying the final accumulated proton pulse by about 1000 relative to a single pulse (see the vignette below on SNS accelerator operation). Protons produced during the $1 \mathrm{msec}$ linac operating period could be transported directly to the STS target without compression, turning STS into a long proton pulse source similar to the European Spallation Source (ESS) but at lower total proton beam power ( $700 \mathrm{~kW}$ at STS compared to $5 \mathrm{MW}$ at ESS), although with a shorter pulse ( 1 msec at STS 
compared to $2.86 \mathrm{msec}$ at ESS). It is important to note that in either long- or short-proton pulse mode, the upgraded accelerator will deliver a maximum energy of $46.7 \mathrm{~kJ} /$ pulse. Figure 2.1 compares the neutron pulses emitted from a high brightness $(3 \mathrm{~cm}$ tall viewed area) cold, coupled para- $\mathrm{H}_{2}$ moderator optimized for both a long proton pulse STS (STSLP) and a short proton pulse STS (STS-SP). The moderator diameter was optimized separately for each source. Using the accumulator ring to provide sharp proton pulses produces a peak brightness $4 \times$ higher than that produced by the STS-LP moderator. On the other hand, with a somewhat larger diameter, the STS-LP moderator produces about 28\% higher time-averaged brightness than the STS-SP moderator. Neutron instruments optimize somewhere between the peak brightness and the time-averaged or integral brightness. This means that an optimized version of an instrument at STSSP would have a performance between 4 and $3 / 4$ times the performance of an optimized instrument at STS-LP. Figure 2.2 shows the peak and average

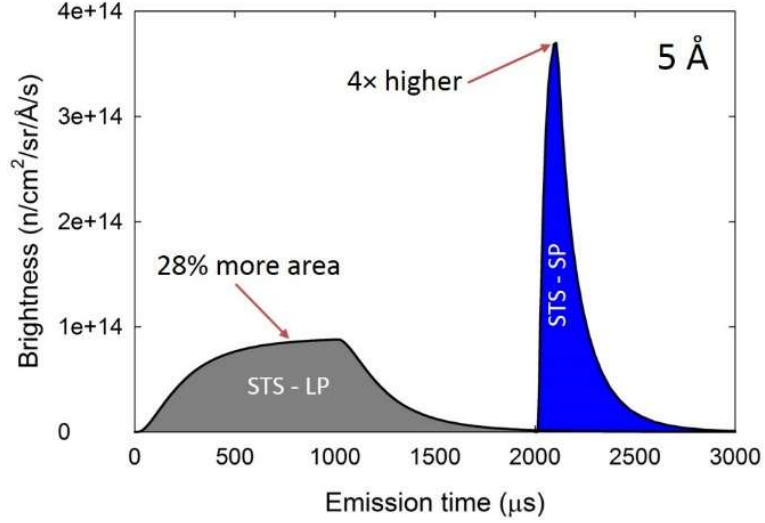

Figure 2.1. Coupled para-hydrogen moderator pulses optimized for long-pulse and short-pulse. $46.7 \mathrm{~kJ}$ proton pulses were used for each calculation. brightnesses as a function of wavelength. Only a few instrument concepts identified for STS could, in principle, make use of the full pulse width at any reasonable instrument length that could be supported on the SNS site. Figure 2.3 shows the wavelength resolution achieved for the indicated instrument lengths that span the range of what can be accommodated on the site. Only low-wavelength resolution instruments like a traditional SANS or Neutron Spin Echo could benefit from the coarse wavelength resolution provided by STS-LP. Consistent with the ORNL three-source strategy, instrument designs that require the highest average brightness are better located on the HFIR cold source, which has about $2 \times$ the time average brightness of the STS-LP moderator, while instruments that optimize to peak brightness are clearly better located on a short proton pulse STS.
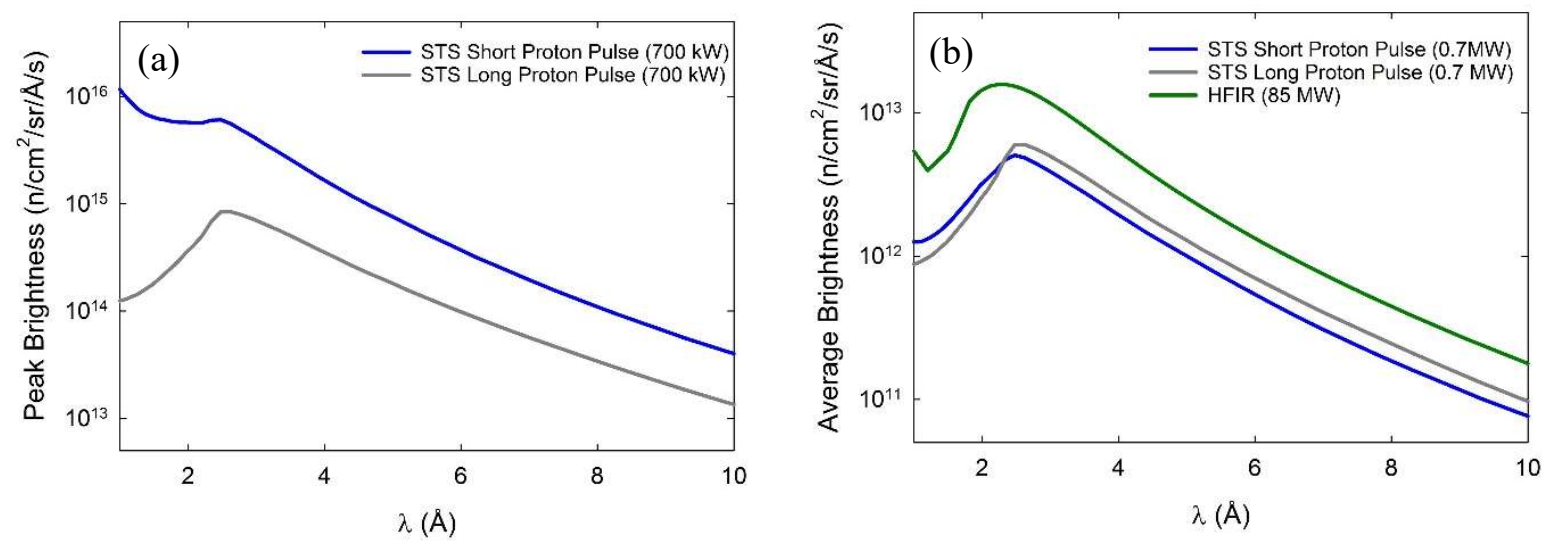

Figure 2.2. Comparison of performance for long and short proton pulse operation of STS cold, coupled para-H2 moderators. (a) shows the peak brightness as a function of neutron wavelength. (b) shows the time averaged brightness and includes the HFIR cold source for reference. 
Finally, the STS science case includes the need for several instruments that are optimally located on a poisoned, de-coupled STS moderator. These include a high-resolution powder diffractometer, a highresolution spectrometer, and several indirect geometry spectrometers that all rely on the high peak brightness and low frequency of STS.

In summary, the choice of operating STS as a short proton pulse neutron source was made for the following reasons:

1. The upgraded SNS accelerator will deliver 46.7 $\mathrm{kJ}$ proton pulses to the STS target in either longor short-proton pulse mode.

2. The increase in total time averaged brightness of $28 \%$ of a larger STS-LP moderator is far

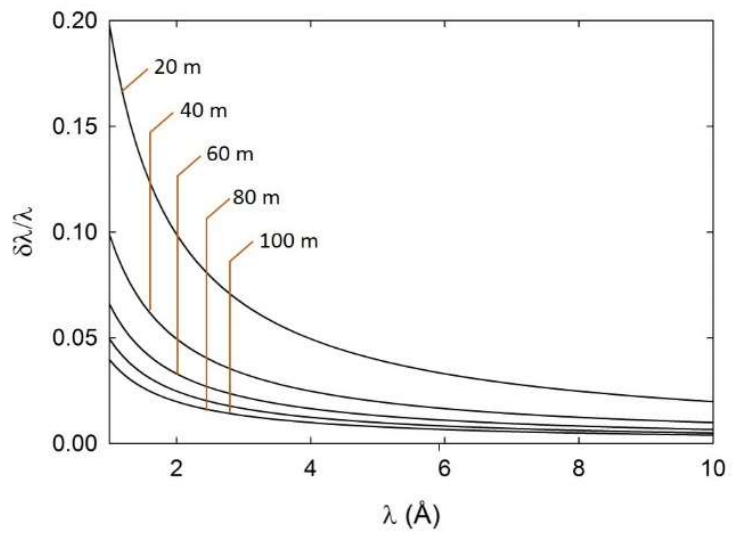

Figure 2.3. Wavelength resolution provided by the 1 msec broad pulse of the STS-LP moderator as a function of neutron wavelength at the indicated instrument lengths. outweighed by the benefits of the $4 \times$ higher peak brightness of the STS-SP moderator optimized for peak brightness.

3. Only a few of the proposed STS instruments can make use of the full pulse width produced by the STS-LP moderator. The majority of the instruments optimize more to peak brightness.

4. A poisoned, de-coupled moderator cannot be supported at a STS-LP source.

5. Instruments that optimize to high time-averaged brightness are better located at HFIR. 


\section{Production of a Short Proton Pulse at the SNS Accelerator}

At SNS, neutrons are produced 60 times a second as a $\sim 1 \mathrm{GeV}$ burst of protons spalls neutrons from the nuclei of a mercury target. The process begins in the front-end where a hydrogen gas plasma creates a pulsed beam of $\mathrm{H}$ - ions that is then accelerated to an energy of $2.5 \mathrm{MeV}$. The ion pulses are further accelerated through four types of linear accelerator to a final energy of $\sim 1 \mathrm{GeV}$. Sequential slices of chopped $\mathrm{H}$ - beam, each about 700 nsec long, pass through a stripper foil that removes the electrons allowing the resultant proton pulses to stack on top of each other in the proton accumulator ring. This process intensifies the final accumulated pulse by a factor of about 1000 compared to a single pulse. After accumulation, the intense proton pulse is kicked out of the accumulator ring and transported to the mercury target where each proton generates about 26 neutrons. The high-energy spallation neutrons are moderated to energies suitable for neutron scattering applications by interacting with hydrogen in moderators located above and below the target. Neutron beam lines are illuminated by neutrons emitted from the moderator faces.

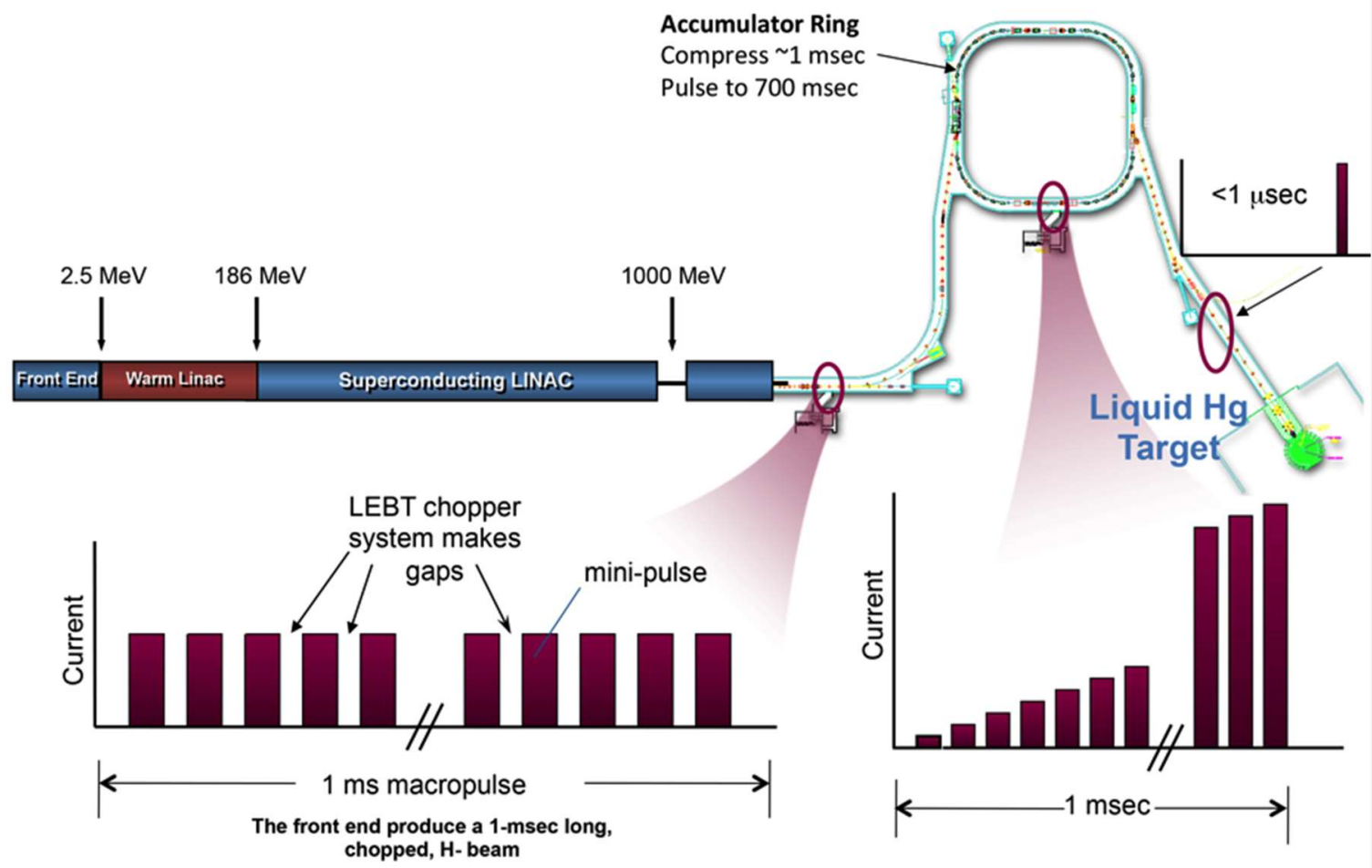

A schematic view of the SNS accelerator and timing system.

\subsection{SOURCE FREQUENCY}

\subsubsection{Introduction}

The decision to change the STS target concept to a solid rotating target in January 2016 presented the opportunity to consider operating the STS at higher power than the $467 \mathrm{~kW}$ being considered up to that point (see section 2.3.2 below). STS operating frequencies between 10 and $20 \mathrm{~Hz}$ with corresponding 
proton beam powers of $467 \mathrm{~kW}$ to $934 \mathrm{~kW}$ have since been considered. It should be noted that the choice represents an optimization between bandwidth and flux (see section 2.3.3 below) over no more than a factor of 2, assuming no loss in neutron production efficiency at higher proton beam power. Under this assumption, $10 \mathrm{~Hz}$ can be effectively implemented by using neutron choppers to suppress intervening frames on a $20 \mathrm{~Hz}$ source. $20 \mathrm{~Hz}$ provides the highest time averaged brightness as a function of wavelength, but does not provide sufficient bandwidth for some instruments at their optimum lengths. Apart from the flux/bandwidth balance, additional trends that are more difficult to quantify tend to favor longer instruments and a corresponding lower source frequency (see section 2.3.5 below). Finally, choice of STS operating frequency impacts operation of FTS instruments, and the various operating modes are discussed in section 2.3.6 below. Table 2.1 summarizes the advantages and disadvantages of the frequency options considered for STS. The choice of $15 \mathrm{~Hz}$ as the STS operating frequency was selected for the following reasons:

1. The choice of $15 \mathrm{~Hz}$ as the STS operating frequency provides superior performance for FTS instruments using frame suppression to operate at an effective frequency of $30 \mathrm{~Hz}$. In this mode, these instruments receive $2 / 3$ of the pulses going to FTS making it effectively equivalent to a $2.7 \mathrm{MW} 60$ $\mathrm{Hz}$ source. $20 \mathrm{~Hz}$ does not provide this gain in FTS instrument performance.

2. $15 \mathrm{~Hz}$ is better than $20 \mathrm{~Hz}$ at supporting kinetic measurements which are a key element of the STS science case.

3. $15 \mathrm{~Hz}$ provides a good balance between flux and bandwidth in optimizing the performance of STS instruments, and with frame suppression provides operating modes of $7.5 \mathrm{~Hz}$ and $15 \mathrm{~Hz}$ at a $700 \mathrm{~kW}$ STS. This option also allows $30 \mathrm{~Hz}$ and 45 pulses/sec at a 2 MW FTS (with 2/3 of the FTS pulses separated by $1 / 60 \mathrm{sec}$ and $1 / 3$ pulses separated by $2 / 60 \mathrm{sec}$ ). In combination across the two facilities, this provides a wide range of choices for future instrument optimization.

4. A number of difficult to quantify considerations such as background, available space, complexity in "stitching" data, and detector limitations tend to favor longer instruments which trend to favoring lower source frequency.

5. At $15 \mathrm{~Hz}$, STS proton pulses will be $46.7 \mathrm{~kJ}$, which is the maximum power/pulse that can be delivered by the accelerator after the Proton Power Upgrade, and FTS proton pulses will be $44.4 \mathrm{~kJ}$, $95 \%$ of maximum nearly maximizing use of the upgraded accelerator.

Table 2.1. Advantages and disadvantages associated with the indicated STS operating frequency

\begin{tabular}{|c|c|c|}
\hline & Advantages & Disadvantages \\
\hline $\begin{array}{c}10 \mathrm{~Hz} \\
(467 \mathrm{~kW})\end{array}$ & $\begin{array}{l}\text { - Second best option for FTS instruments } \\
\text { desiring to run in frame suppression mode } \\
\text { at an effective FTS frequency of } 30 \mathrm{~Hz} \\
\text { equivalent to a } 2.4 \mathrm{MW} 60 \mathrm{~Hz} \text { FTS. } \\
\text { B } \text { Broad dynamic range benefits the } \\
\text { BWAVES instrument concept. } \\
\text { - Lowest energy proton pulses delivered to } \\
\text { FTS mercury target (lowered demand on } \\
\text { mitigating cavitation induced erosion). } \\
\text { - Lowest time-averaged power delivered to } \\
\text { the STS results in longest target/moderator } \\
\text { component lifetime saving on replacement } \\
\text { costs }(\approx \$ 1.4 \mathrm{M} \text { to } \$ 2.3 \mathrm{M} / \text { year relative to } 20 \\
\text { Hz operation) }\end{array}$ & $\begin{array}{l}\text { Essentially duplicates } 20 \mathrm{~Hz} \text { frame suppression } \\
\text { mode while limiting flux for STS instruments } \\
\text { that can operate at higher frequency. } \\
\text { - Leaves excessive accelerator operating margin } \\
\text { (unused power). } \\
\text { - Lowest time averaged brightness delivered to } \\
\text { STS instruments. } \\
\text { - Provides excessive bandwidth to direct } \\
\text { geometry spectrometers, CHESS and HERTZ, } \\
\text { even when repetition rate multiplication is } \\
\text { considered. }\end{array}$ \\
\hline
\end{tabular}


Table 2.1. Advantages and disadvantages associated with the indicated STS operating frequency (continued)

\begin{tabular}{|c|c|c|}
\hline & Advantages & Disadvantages \\
\hline $\begin{array}{c}15 \mathrm{~Hz} \\
(700 \mathrm{~kW})\end{array}$ & $\begin{array}{l}\text { Best choice for FTS instruments desiring to } \\
\text { run in frame suppression mode at an } \\
\text { effective FTS frequency of } 30 \mathrm{~Hz} \\
\text { equivalent to a } 2.7 \mathrm{MW} 60 \mathrm{~Hz} \mathrm{FTS} \text {. } \\
\text { - } 50 \% \text { higher time-averaged neutron } \\
\text { brightness as a function of wavelength } \\
\text { across } 2 / 3 \text { the bandwidth as } 10 \mathrm{~Hz} \text {. } \\
\text { - } \text { Best choice for QIKR reflectometer and } \\
\text { provides time-resolved capability for SANS } \\
\text { instruments (when frame suppression is } \\
\text { used to provide an effective } 7.5 \mathrm{~Hz} \\
\text { equivalent bandwidth). } \\
\text { - Provides wide range of choices for future } \\
\text { instrument operation - } 7.5 \mathrm{~Hz} \text { and } 15 \mathrm{~Hz} \text { at } \\
\text { STS; } 30 \mathrm{~Hz} \text { and } 45 \text { pulses/sec at FTS } \\
\text { (including frame suppression modes). } \\
\text { Uses all but } 3.6 \% \text { of the maximum } \\
\text { accelerator power delivered by the Proton } \\
\text { Power Upgrade project. }\end{array}$ & $\begin{array}{l}\text { - } 33 \% \text { less time averaged neutron brightness as a } \\
\text { function of wavelength than } 20 \mathrm{~Hz} \text {. } \\
\text { Provides more bandwidth to the direct } \\
\text { geometry spectrometers than can be usefully } \\
\text { employed. }\end{array}$ \\
\hline $\begin{array}{c}20 \mathrm{~Hz} \\
(933 \mathrm{~kW})\end{array}$ & $\begin{array}{l}\text { - Highest time-averaged neutron brightness } \\
\text { as a function of wavelength. ( } 33 \% \text { higher } \\
\text { across } 3 / 4 \text { of the bandwidth as } 15 \mathrm{~Hz}) \text {. } \\
\text { - Best choice for direct geometry } \\
\text { spectrometers CHESS and HERTZ. } \\
\text { - Uses all the proton beam power delivered } \\
\text { by the Proton Power Upgrade Project. } \\
\text { - Essentially provides equivalent } 10 \mathrm{~Hz} \\
\text { operation when operating in frame } \\
\text { suppression mode. }\end{array}$ & $\begin{array}{l}\text { - In FTS frame suppression mode, suppresses } 1 \\
\text { out of } 2 \text { pulses reducing the performance by } \\
43 \% \text { relative to } 15 \mathrm{~Hz} \text { operation. } \\
\text { - Highest energy proton pulses delivered to FTS } \\
\text { mercury target places a higher demand on } \\
\text { mitigating cavitation induced erosion. } \\
\text { - Highest time-averaged power delivered to STS } \\
\text { target results in shortest target/moderator } \\
\text { component lifetime with higher operating costs } \\
\text { associated with component replacement ( } \approx \\
\text { \$1.4M to } \$ 2.3 \mathrm{M} / \text { year relative to } 10 \mathrm{~Hz} \\
\text { operation). } \\
\text { Delivers slightly less power to FTS than the } \\
\text { maximum it was designed to receive. } \\
\text { High frequency tends to favor shorter } \\
\text { instrument design which pose challenges in } \\
\text { optics designs, background reduction, and data } \\
\text { "stitching" }\end{array}$ \\
\hline
\end{tabular}

\subsubsection{STS Target Evolution}

The 2014 target design in the Technical Design Report (TDR) was a conventional, fixed, compact, solid target composed of tungsten plates as neutronics calculations had shown that this concept would perform well with beam power on the order of $500 \mathrm{~kW}[2,3]$. In early 2016, the project accepted an expert review committee's recommendation and replaced the stationary target with a rotating target concept, in large part, driven by accident scenario concerns [4,5]. Initial neutronic analysis of the rotating target had an acceptable worst-case accident scenario, neutron production equivalent to the stationary target, and an extended lifetime. The rotating target also presented the opportunity to increase the power delivered to STS up to approximately $1 \mathrm{MW}$. The original $10 \mathrm{~Hz}, 467 \mathrm{~kW}$ STS concept described in the TDR already used the maximum proton energy/pulse that the upgraded accelerator could deliver, so increasing STS 
power means considering the impacts of increasing the STS frequency. More details are found in Section 5.3.4.

\subsubsection{Bandwidth, Resolution and Instrument Length}

Pulsed neutron sources (and some instruments at continuous, reactor sources) use time-of-flight methods to determine the neutron wavelength. The time $t$ for a neutron of wavelength $\lambda$ to travel a distance $L$ is

$$
t=2.528 \cdot 10^{-4} \lambda L
$$

for $t$ in seconds, $\lambda$ in $\AA$, and $L$ in meters. The uncertainty in determining the neutron wavelength depends on the uncertainty in $L$ (which is typically very small by design and will be ignored below) and the width of the moderator pulse emitted at the face of the moderator, $\delta t$, often taken as the full-width-at-halfmaximum, so that the wavelength resolution $\delta \lambda$ is

$$
\delta \lambda=\delta t /\left(2.528 \cdot 10^{-4} L\right)
$$

At a short proton pulse neutron source like STS, higher wavelength resolution can be obtained through reducing $\delta t$ in design of the moderator and/or increasing instrument length. However, designing a moderator with sharp pulses inevitably lowers the neutron flux emitted by the moderator. High-speed neutron choppers (rotating disks made of neutron absorbing material with open slots) can define a narrower pulse than created by the neutron source, but with a reduction in bandwidth (see below). This approach is being taken for many European Spallation Source instruments, as its intrinsic moderator pulse width is too broad to provide the required $\delta \lambda$ at reasonable instrument length.

The wavelength bandwidth, $\Delta \lambda$, that can be used without fast neutrons from a subsequent pulse overlapping in time at the detector with slower neutrons from the previous pulse is largely determined by the time between source pulses (the frame width)

$$
\Delta \lambda=1 /\left(2.528 \cdot 10^{-4} L f\right)
$$

where $f$ is the source operating frequency (ignoring the reduction by the moderator pulse width, $\delta t$, as it is much less than $1 / f$ at a short proton pulse source). In practice, neutron choppers have a finite opening and closing time as the aperture sweeps through the beam width (or height depending on the orientation of the chopper), limiting the bandwidth that can be used to approximately $90 \%$ of $\Delta \lambda$ depending on the specifics of the individual instrument design. Bandwidth is increased by operating the source at a lower frequency or shortening instruments. Neutron choppers can be used to suppress intervening source pulses, effectively lowering the source frequency and increasing $\Delta \lambda$ but with a corresponding reduction in the flux of neutrons across the wavelength band used at higher frequencies as illustrated in Figure 2.4. In the example illustrated in Figure 2.4, the wavelength band between 1 and $4.3 \AA$ illuminated at $60 \mathrm{~Hz}$, receives $1 / 2$ of the neutrons when every other frame is suppressed, however neutrons with wavelengths between 4.3 $\AA$ and $7.7 \AA$ are now present. The relative value of any neutron wavelength to the measurement depends on the instrument and details of the experiment (see section 2.3.6 below). Frame suppression typically, but not always, results in a relatively short dead time at the detector when backgrounds rise due to incomplete blockage of high energy neutrons produced at the start of the suppressed pulse. 
Instrument and source design is thus an optimization of $\delta t, L$, and $f$. In general, longer instruments have higher wavelength resolution, while shorter instruments have a broader wavelength bandwidth. A lower source frequency (or use of frame suppression) provides access to a broader wavelength bandwidth, but fewer pulses will mean lower neutron flux over part of the wavelength band as illustrated for the SNS first target station in Figure 2.4.

\subsubsection{Constraints on STS Frequency and Assumptions}

- Both FTS and STS must operate at fixed frequencies due to the use of periodic devices, primarily rotating neutron choppers. The SNS accelerator operates at $60 \mathrm{~Hz}$ with a $6 \%$ linac duty cycle (see vignette on SNS accelerator and Section 2.1.1). The most efficient and lowest cost way to split pulses between FTS and STS is to operate in "pulse-stealing" mode where STS takes a periodic subset of the proton pulses with the remainder going to FTS. This means that FTS will operate with some pulses $2 / 60 \mathrm{sec}$ apart and some pulses $1 / 60 \mathrm{sec}$ apart.

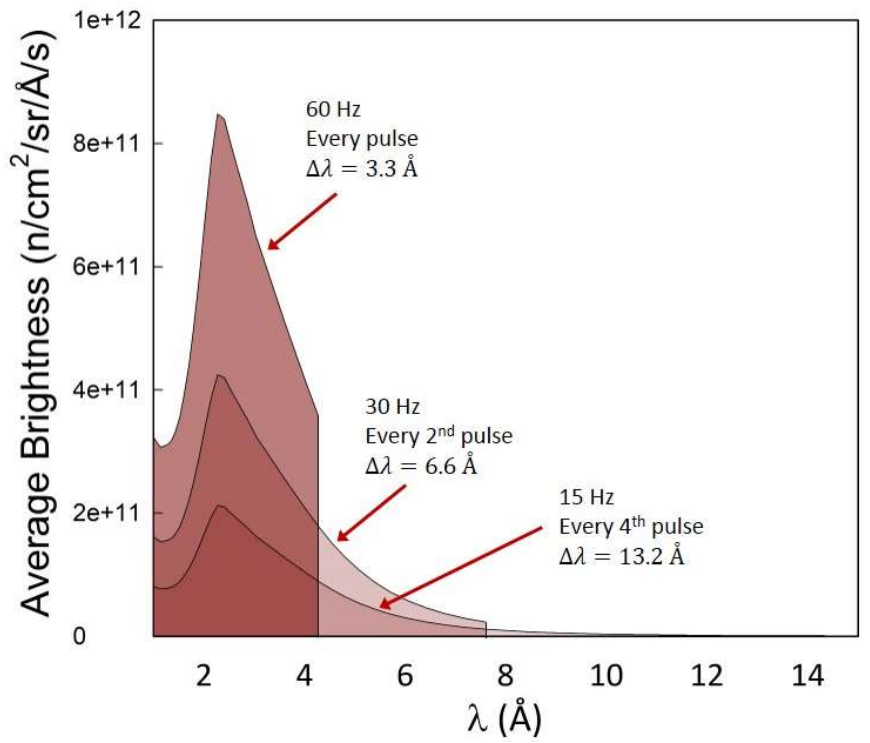

Figure 2.4. Time averaged neutron brightness when frame suppression is used to effectively broaden the neutron wavelength bandwidth. A representative instrument length of $20 \mathrm{~m}$ was chosen to create the plot with a minimum wavelength of $1 \AA$ and maximum wavelength set by the bandwidth at the indicated operating frequency. The moderator is the FTS coupled para-hydrogen moderator at 1.4 MW.

- The highest energy proton pulses the accelerator can deliver to either FTS or STS is $46.7 \mathrm{~kJ}$. However, the accelerator can send proton pulses of less than the maximum $46.7 \mathrm{~kJ} /$ pulse energy to either of the target stations, essentially by controlling how many protons are in each pulse. STS will always receive $46.7 \mathrm{~kJ}$ proton pulses.

- The maximum power that can be delivered to FTS is $2 \mathrm{MW}$, regardless of how many proton pulses/sec it receives [2]. This limit was established in the original design requirements of the FTS target and instruments, and infrastructure such as target cooling systems and instrument shielding were designed accordingly. At lower frequencies, this places a more severe demand on the mercury target as the energy/pulse is increased. As noted above, the accelerator will be able to tailor the energy/pulse received by STS and FTS targets independently, so an optimal FTS energy/pulse can be implemented.

- We assume here that there is no loss in STS neutron production efficiency as measured by moderator brightness with increasing proton power. That is, moderator neutron pulses are identical if produced at $10 \mathrm{~Hz}, 15 \mathrm{~Hz}$ or $20 \mathrm{~Hz}$. If practical considerations such as a need for increased cooling at higher power impose a significant decrease in neutron production efficiency, this assumption will have to be reexamined. To date there is no expectation that this will occur. 
- We also assume that there is no loss in FTS neutron production efficiency as experienced on the neutron beam lines as the energy per proton pulse is increased up to the maximum while maintaining a total power of $2 \mathrm{MW}$.

- Based on experience at FTS, operating chopper systems to suppress some number of intervening pulses is effective and will be viable at STS. Some current instruments take advantage of this mode of operation (see Table 2.3 below.)

\subsubsection{Instrument and Source Trends}

As noted above, instrument and source design depends on optimization of moderator characteristics, instrument length and source frequency. There are many practical considerations, including some that are difficult to quantify that trend to favoring longer instruments and a correspondingly lower operating frequency to provide adequate band width at longer distances. Alternatively, some considerations tend to favor shorter instruments and a correspondingly higher operating frequency will provide adequate band width. A brief discussion of some of these topics is given below:

1. Instrument length plays a significant role in selection of the neutron optics designed to deliver the optimum neutron phase space (and ideally no more) to the sample position. Choices are relatively limited for short instruments (e.g. "point-to-point" focusing mirror systems such as proposed for the STS Variable Beam Profile Reflectometer require instrument lengths $\approx 40 \mathrm{~m}$ or longer as demonstrated by the European Spallation Source Estia reflectometer design [6].) Similar concepts have been proposed for the STS EWALD diffractometer and MENUS engineering materials station, but these instruments are already at long distances to achieve the desired wavelength resolution and benefit from an intermediate operating frequency.

2. In general, too low a source frequency might result in excess bandwidth for short instruments, while too high a source frequency might require long instruments to "stitch" data across multiple instrument configurations or operate at effectively lower frequency using choppers to suppress neutron pulses. The STS planning suite of instruments (see Appendix A and Table 2.5) has a balance of long and short instruments as reflected in the site layout. $15 \mathrm{~Hz}$ provides a good balance between the needs of long and short instruments.

3. Longer instruments tend to have better access to the sample area which will be critical at STS. The STS science case has an emphasis on integrating multiple characterization techniques with neutron scattering to probe complex materials and transient behavior [7]. With a nominal $11 \mathrm{deg}$ beam separation, the average lateral distance (perpendicular to the neutron beam) available to an instrument is $0.19 \times \mathrm{D}$ where $\mathrm{D}$ is the distance from the moderator. Initial shielding calculations show that within about $30 \mathrm{~m}$, straight instruments will require about $50 \mathrm{~cm}$ to $75 \mathrm{~cm}$ of high density concrete to shield the beam line as illustrated in Figure 5.61. (Strongly curved beam lines may require somewhat less shielding). Some of this shielding thickness can likely be shared with a neighboring beam line, but an estimate of the lateral space available would be $0.19 \times \mathrm{D}-1 \mathrm{~m}$. At a sample distance of about $18.5 \mathrm{~m}$, the kinetics reflectometer QIKR could expect about $2.5 \mathrm{~m}$ of lateral distance around the sample (or more depending on geometry of adjacent instruments). Building instruments much shorter than this (to support higher source frequency) will require careful integration with neighboring beam lines and will be challenging.

4. In general, achieving low backgrounds is easier on longer instruments than shorter ones. Distance reduces instrument detector view of the source and provides more options and space for inserting shielding between the target monolith and the working end of the instrument. 
5. Neutron band width choppers tend to get smaller in diameter at higher source frequencies as illustrated in Figure 5.57 if the requirement is to maintain the same open/close times as the chopper blade sweeps through the neutron beam. Maintaining that parameter means larger blade diameters when the chopper spins at lower frequency. Larger choppers are more challenging to integrate into the beam lines, particularly within $10 \mathrm{~m}$ from the moderators.

6. Shorter instruments tend to place a higher demand on instantaneous count rates on detectors. This can be a technical challenge (e.g. even on current reflectometers).

7. Shorter beam lines tend to cost less than longer beam lines that require more neutron guide, more beam line shielding, and may require an external building with its associated cost. As noted already though, the STS is approximately balanced between long and short instruments with long instruments requiring the better wavelength resolution afforded by additional distance.

8. Transport of shorter-wavelength neutrons is more efficient on shorter beam lines.

9. Higher source frequency may require more use of data "stitching" where multiple data sets collected at different instrument configurations or settings are merged together to form a complete data set. Data-"stitching" presents different complications for different instrument types depending on subtleties like possible dead time while changing instrument configurations, changing backgrounds with new instrument settings, proper normalization, and changing instrument resolution. Sorting through these issues can be time consuming, hindering instrument productivity in its early, critical stages. Alternatively, frame suppression can be employed to provide an effective lower source frequency. In the latter case, the higher source frequency is of no value and may even be a bit of a detriment due to detector dead time during the initial period of the masked pulse.

10. Some wavelength dependent optical components are difficult to implement or optimize over the large wavelength bands associated with lower source frequency. A prime example was presented by a reflectometer working group (Section 2.3.6.2 below) that expressed uncertainty in the ability to effectively enable polarization analysis across a broad wavelength band without significant loss of intensity.

11. Repetition rate multiplication partially compensates for lower source frequencies on direct geometry spectrometers like the STS CHESS and HERTZ instruments. However, as the incident neutron energy is lowered, an increasingly wider part of the time frame is required to support inelastic studies. At some point, as source frequency is lowered, a point of diminishing returns is reached where the lowest incident energy requires more of the time frame than is warranted by the additional information generated. In this case, the instrument would prefer to operate at a higher frequency and collect data at more useful incident energies at a higher rate.

Choice of band width has some general implications for source operation. The increase in proton operating frequency from $10 \mathrm{~Hz}$ to $15 \mathrm{~Hz}$ did not require significant changes in the design of the target station or core components. Importantly, the proposed rotating target had sufficient design margin to accommodate the additional heating and radiation dose. The relatively modest increase in component heating was also within the conceptual design limit for the surrounding core components although the increase in the radiation damage to the proton beam window and moderator/reflector assembly will require more frequent replacements at the higher power (higher frequency) levels. For example, the proton beam window that isolates the accelerator from the vessel environment surrounding the target has a design life estimated at 1.1 year based on helium damage and 6.5 years based on dpa damage at $20 \mathrm{~Hz}$ operation (proportionally longer at lower frequencies). However, this is a conservative approach and depends on the underlying design and operating criteria that remain to be established. At approximately $\$ 1 \mathrm{M}$ per replacement, the additional cost 
of operating at $20 \mathrm{~Hz}$ rather than $10 \mathrm{~Hz}$, is approximately $\$ 1 \mathrm{M} /$ replacement $=\$ 153 \mathrm{k}$ to $\$ 1.1 \mathrm{M} /$ year. The moderator/reflector assembly is anticipated to be replaced every 1.6 years at $20 \mathrm{~Hz}$. At an approximate cost of about $\$ 2 \mathrm{M}$, the additional cost associated with this component operating at $20 \mathrm{~Hz}$ rather than $10 \mathrm{~Hz}$, is approximately $\$ 2 \mathrm{M} / 1.6$ years $=\$ 1.25 \mathrm{M} /$ year. The target itself has a theoretical life time in excess of 20 years but is planned for replacement every 10 years, irrespective of proton beam power.

\subsubsection{Operating Modes}

In pulse-stealing mode, FTS operates with minimum time between pulses of $1 / 60 \mathrm{~s}$ as it does now, but with twice that time when the intervening pulse is directed to STS. STS thus operates at a fixed frequency that is a sub-multiple of 60 while FTS receives the remaining pulses but does not operate with fixed time between pulses. Table 2.2 describes some of the possible operating modes. The strategy is to always deliver full energy proton pulses to the STS target, and adjust the energy of the FTS proton pulses up to the maximum $46.7 \mathrm{~kJ} /$ pulse to deliver a maximum power of $2 \mathrm{MW}$ to maintain the performance of FTS instruments. All other things being equal, STS at 15 or $20 \mathrm{~Hz}$, with the remaining pulses going to FTS at the indicated energy levels appear to be the most effective at using the full accelerator power delivered by the Proton Power Upgrade. A $15 \mathrm{~Hz}$ STS leaves about $100 \mathrm{~kW}$ operating margin for the accelerator, while a $20 \mathrm{~Hz}$ STS prevents FTS from quite reaching $2 \mathrm{MW}$ but the difference is small and likely inconsequential to the quality of FTS measurements. (It is possible that operational experience and subsequent evaluation might find enough margin to raise FTS power to 2.1 MW and enable full energy pulses to be delivered to FTS at $45 \mathrm{~Hz}$.)

Table 2.2. Operation of FTS and STS in pulse-stealing mode. It is assumed that regardless of operating frequency, STS will accept full energy proton pulses of $46.7 \mathrm{~kJ}$.

\begin{tabular}{cccccc}
\hline $\begin{array}{c}\text { STS } \\
(\mathbf{H z})\end{array}$ & $\begin{array}{c}\text { STS Power } \\
(\mathbf{M W})\end{array}$ & $\begin{array}{c}\text { FTS } \\
\text { (pulses/sec) }\end{array}$ & $\begin{array}{c}\text { FTS energy/pulse } \\
(\mathbf{k J})\end{array}$ & $\begin{array}{c}\text { FTS Power } \\
(\mathbf{M W})\end{array}$ & $\begin{array}{c}\text { Total Power } \\
(\mathbf{M W})\end{array}$ \\
\hline 5 & 0.233 & 55 & 36.4 & 2.0 & 2.233 \\
10 & 0.467 & 50 & 40 & 2.0 & 2.467 \\
15 & 0.700 & 45 & 44.4 & 2.0 & 2.7 \\
20 & 0.933 & 40 & 46.7 & 1.867 & 2.8 \\
\hline
\end{tabular}

\subsubsection{Impacts on FTS instrument operation}

Some of the current FTS instruments as well as some proposed for STS want the ability to operate in frame suppression mode, using choppers to suppress unwanted, intervening pulses and increasing $\Delta \lambda$. Table 2.3 summarizes a survey (January 2017) of the current FTS instrument use of frame suppression. As can be seen, 9 beam lines use frame suppression to operate at lower frequency, and 6 make significant use $(\geq 15 \%)$ of this option. 
Table 2.3. FTS instrument operation in frame suppression mode

\begin{tabular}{|c|c|c|}
\hline Beam line & $\begin{array}{c}\text { Operating } \\
\text { Frequency }(\mathbf{H z})\end{array}$ & $\begin{array}{c}\text { Fraction } \\
(\%)\end{array}$ \\
\hline BL-1A USANS & 60 & 100 \\
\hline BL-1B NOMAD & $\begin{array}{l}30 \\
60\end{array}$ & $\begin{array}{c}<5 \\
>95\end{array}$ \\
\hline BL-2 BASIS & $\begin{array}{l}30 \\
60\end{array}$ & $\begin{array}{l}30 \\
70\end{array}$ \\
\hline BL-3 SNAP & $\begin{array}{l}30 \\
60\end{array}$ & $\begin{array}{l}<10 \\
>90\end{array}$ \\
\hline *BL-4A Mag. Ref. & 30 & 100 \\
\hline *BL-4B Liq. Ref. & $\begin{array}{l}30 \\
60\end{array}$ & $\begin{array}{l}>80 \\
<20\end{array}$ \\
\hline BL-5 CNCS & 60 & 100 \\
\hline${ }^{\dagger} \mathrm{BL}-6 \mathrm{EQ}-\mathrm{SANS}$ & 60 & 100 \\
\hline BL-7 VULCAN & $\begin{array}{l}20 \\
30 \\
60\end{array}$ & $\begin{array}{l}20 \\
60 \\
20\end{array}$ \\
\hline BL-9 CORELLI & 60 & 100 \\
\hline BL-11A POWGEN & 60 & 100 \\
\hline BL-11B MaNDi & $\begin{array}{l}30 \\
60\end{array}$ & $\begin{array}{l}15 \\
85\end{array}$ \\
\hline BL-12 TOPAZ & 60 & 100 \\
\hline BL-13 FNPB & 60 & 100 \\
\hline BL-14B HYSPEC & 60 & 100 \\
\hline BL-15 NSE & 60 & 100 \\
\hline BL-16B VISION & 30 & 100 \\
\hline BL-17 SEQUOIA & 60 & 100 \\
\hline BL-18 ARCS & 60 & 100 \\
\hline
\end{tabular}

As demonstrated in Table 2.3, it is important to understand the possible impacts of STS operating frequency on FTS instruments that operate with frame suppression. Some of these techniques might ultimately be better served with an STS instrument, but it is unlikely that all will move to STS when it first operates. In addition, one of the operating modes (15 Hz STS) illustrated below provides a superior option for FTS instruments that operate with frame suppression and more of the existing instruments and certainly future FTS instrument concepts might well desire to employ this option. Figure 2.5 illustrates the $10 \mathrm{~Hz}, 15 \mathrm{~Hz}$ and $20 \mathrm{~Hz}$ STS operating mode listed in Table 2.2. For STS at $10 \mathrm{~Hz}$, frame suppression on FTS works well, with no missing pulses, so a true $30 \mathrm{~Hz}$ FTS mode is preserved that is supported by 3 of 5 FTS pulses. There is one special frame where the choppers would be closed but when beam is directed to STS. This situation is also true for STS at $15 \mathrm{~Hz}$, but more special frames exist and a higher fraction (2/3) of FTS pulses support its $30 \mathrm{~Hz}$ mode. At $20 \mathrm{~Hz}$, a true $30 \mathrm{~Hz}$ operation of FTS in frame suppression mode is no longer possible. Some pulses (indicated with a $\square$ in Figure 2.5) required for $30 \mathrm{~Hz}$ FTS mode are missing as they are directed to STS. In this case, FTS operates at $20 \mathrm{pulses} / \mathrm{sec}$ in frame 
suppression mode with $1 / 2$ of the pulses separated by $2 / 60 \mathrm{sec}$ and the remainder by $4 / 60 \mathrm{sec}$. The proton power that supports each of these modes is listed in Table 2.4. For example, at $10 \mathrm{~Hz}, 3 / 5$ of the total power delivered to FTS supports its $30 \mathrm{~Hz}$ frame suppression operation. Note that the shortest time between pulses is still 1/60 sec for FTS regardless of the number of proton pulses per second (pps) directed to its target, and in frame suppression mode, the shortest time between frames is $2 / 60 \mathrm{sec}$. With STS at $15 \mathrm{~Hz}$, instruments operating in frame suppression mode on FTS receive $43 \%$ more neutrons than when STS operates at $20 \mathrm{~Hz}$. (The limiting scenario would occur when choppers at FTS suppress only pulses that are already directed to STS. However, in this case both sources would be effectively operating at $30 \mathrm{~Hz}$, and the accelerator would deliver 1.4 MW to each source. This would be a loss for FTS instruments that can operate effectively at higher frequency and only provide $5.3 \%$ more power than is already available in the STS $15 \mathrm{~Hz}$ mode described in Table 2.4). 


\begin{tabular}{|c|c|c|c|c|c|c|c|c|c|c|c|c|c|c|c|c|c|c|c|c|c|}
\hline $\begin{array}{r}\text { Current } \\
\text { FTS }(60 \mathrm{~Hz}) \\
\text { FTS frame suppression }(30 \mathrm{~Hz})\end{array}$ & i & $\stackrel{1}{\theta}$ & i & $\theta$ & 1 & Q & i & \& & i & 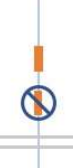 & i & $\dot{\theta}$ & 1 & $\begin{array}{l}\text { I } \mathrm{Ac} \\
\text { I } \mathrm{Ac} \\
\text { Q } \mathrm{Ne}\end{array}$ & $\begin{array}{l}\text { cele } \\
\text { cele }\end{array}$ & $\begin{array}{l}\text { ator } \\
\text { ator } \\
\text { n che }\end{array}$ & $\begin{array}{l}\text { pulse } \\
\text { pulse } \\
\text { pper }\end{array}$ & $\begin{array}{l}\text { es, dir } \\
\text { es, dir } \\
\text { bloc }\end{array}$ & $\begin{array}{l}\text { ecte } \\
\text { ecte } \\
\text { king }\end{array}$ & $\begin{array}{l}\text { d to } \\
\text { d to } \\
\text { pulse }\end{array}$ & \\
\hline $\begin{array}{r}10 \mathrm{~Hz} \text { Pulse Stealing } \\
\text { FTS (50 pps, } 1 / 60 \mathrm{~s} \text { frame }) \\
\text { FTS frame suppression }(30 \mathrm{~Hz}) \\
\text { STS }(10 \mathrm{~Hz})\end{array}$ & i & $\begin{array}{c}\theta \\
1\end{array}$ & 1 & $\theta$ & 1 & 0 & 1 & $\begin{array}{c}\theta \\
1\end{array}$ & I & Q & I & $\theta$ & I & $\begin{array}{l}\theta \\
1\end{array}$ & 1 & $\theta$ & I & 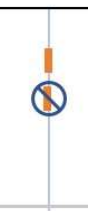 & i & $\begin{array}{c}\theta \\
\mathbf{1}\end{array}$ & 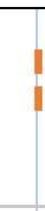 \\
\hline $\begin{array}{r}15 \mathrm{~Hz} \text { Pulse Stealing } \\
\text { FTS (45 pps, } 1 / 60 \text { s frame) } \\
\text { FTS frame suppression }(30 \mathrm{~Hz}) \\
\text { STS }(15 \mathrm{~Hz})\end{array}$ & i & $\begin{array}{c}\theta \\
\mathbf{1}\end{array}$ & i & $\theta$ & i & $\begin{array}{c}\theta \\
\mathbf{I}\end{array}$ & I & 1 & I & $\begin{array}{c}\theta \\
\mathbf{1}\end{array}$ & I & $\hat{\theta}$ & I & $\begin{array}{c}\theta \\
\mathbf{I}\end{array}$ & i & $\theta$ & i & $\begin{array}{c}Q \\
1\end{array}$ & i & $\theta$ & i \\
\hline $\begin{array}{r}20 \mathrm{~Hz} \text { Pulse Stealing } \\
\text { FTS (40 pps, } 1 / 60 \mathrm{~s} \text { frame) } \\
\text { FTS frame suppression (20 pps, } \\
1 / 30 \mathrm{~s} \text { frame }) \\
\text { STS }(20 \mathrm{~Hz})\end{array}$ & 1 & $\theta$ & 1 & $\theta$ & $\square$ & $\theta$ & 1 & Q & 1 & $\theta$ & $\square$ & $\theta$ & 1 & Q & 1 & $\theta$ & $\square$ & $\theta$ & 1 & Q & I \\
\hline & 0 & $\frac{1}{60}$ & $\frac{2}{60}$ & $\frac{3}{60}$ & $\frac{4}{60}$ & $\frac{5}{60}$ & $\frac{6}{60}$ & $\frac{7}{60}$ & $\frac{8}{60}$ & $\frac{9}{60}$ & $\frac{10}{60}$ & $\frac{11}{60}$ & $\frac{12}{60}$ & $\frac{13}{60}$ & $\frac{14}{60}$ & $\frac{15}{60}$ & $\frac{16}{60}$ & $\frac{17}{60}$ & $\frac{18}{60}$ & $\frac{19}{60}$ & $\frac{2 c}{6 c}$ \\
\hline
\end{tabular}

Figure 2.5. Graphical illustration of the operating rhythms for STS and FTS at relevant STS operating frequencies of $10 \mathrm{~Hz}, 15 \mathrm{~Hz}$, and $20 \mathrm{~Hz}$ as indicated in vertical sections 2-4 above. The current condition with FTS operating at $60 \mathrm{~Hz}$ is illustrated at the top of the graph. The horizontal axis is graduated in 1/60 sec to represent the operating frequency of the SNS accelerator. Vertical light blue lines indicate times when the accelerator can deliver a proton pulse to either the first or second target stations. Vertical orange bars indicate proton beam delivery to the FTS target while vertical dark blue bars indicate when proton pulses are delivered to the STS target. The "no"-symbol $(\theta)$ indicates pulses that would be blocked for FTS instrument neutron chopper systems to facilitate frame suppression on FTS. The "व" symbol indicates pulses that would have been ideally directed to FTS to support frame suppression mode but instead are directed to STS. 
Table 2.4. Operating modes of FTS instruments when STS operates at the indicated frequencies. The last column lists the part of the proton power directed to FTS that is used in FTS frame suppression mode.

\begin{tabular}{ccccc}
\cline { 3 - 4 } STS Rate & FTS Rate & $\begin{array}{c}\text { FTS Power } \\
\text { (MW) }\end{array}$ & FTS Rate & Power (MW) \\
\hline & $60 \mathrm{~Hz}$ & 2 & $30 \mathrm{~Hz}$ & 1 \\
$10 \mathrm{~Hz}$ & $50 \mathrm{pps}$ & 2 & $30 \mathrm{~Hz}$ & 1.2 \\
$15 \mathrm{~Hz}$ & $45 \mathrm{pps}$ & 2 & $30 \mathrm{~Hz}$ & 1.33 \\
$20 \mathrm{~Hz}$ & $40 \mathrm{pps}$ & 1.867 & $20 \mathrm{pps}$ & 0.93 \\
\hline
\end{tabular}

\subsubsection{Impacts on STS Instruments}

With the assumption of effective frame suppression, instruments at the STS can choose to run at submultiples of the primary STS frequency. Experience at FTS has demonstrated that even on instruments as short as $15.1 \mathrm{~m}$ (moderator to detector), neutron chopper systems can be effectively spun at $1 / 2$ the FTS 60 $\mathrm{Hz}$ to provide effective $30 \mathrm{~Hz}$ operation with acceptable instrument background. Assuming no loss in neutron production efficiency with higher repetition rate at STS means that instruments can effectively trade bandwidth for flux over a narrower band as illustrated in Figure 2.4. Under this set of assumptions, a $20 \mathrm{~Hz}$ STS with frame suppression supports $10 \mathrm{~Hz}$ operation equally well as a $10 \mathrm{~Hz}$ STS (apart from an anticipated short duration of high background at the start of the masked pulse), effectively eliminating 10 $\mathrm{Hz}$ as the optimum choice for STS based on STS instrument considerations. In addition, instruments that might nominally use $10 \mathrm{~Hz}$ to measure a full data set, might prefer the option to narrow the region of interest at effectively higher neutron flux for particular experiments.

As noted above, instrument optimization is a matching exercise between moderator pulse widths, instrument length and source frequency. The optimum choice delivers the desired wavelength resolution across the desired bandwidth. In practice, there is nearly always some flexibility in what is actually required for resolution and bandwidth and consequently the choice of instrument length and source frequency can be adapted across a reasonable range. The maximum difference in bandwidth and flux for a given instrument length at $15 \mathrm{~Hz}$ or $20 \mathrm{~Hz}$ is $30 \%$. While a $30 \%$ flux increase is always useful, there are situations where a 30\% increase in bandwidth can be critical. The first is when data-"stitching" (piecing together data collected from multiple instrument configurations) is not viable and a full dynamic range must be measured simultaneously (e.g. when the sample is changing during the course of the experiment in a time that is short compared to reconfiguring the instrument). These time-resolved, kinetic measurements are a key element of the STS science case [7]. A prime example is presented by the kinetics reflectometer QIKR that requires $\Delta \lambda$ of about $26 \AA$ (accounting for a $10 \%$ effective loss due to finite chopper open and close times) to simultaneously measure a decade in momentum transfer. At this bandwidth. the total length to the detector is then $15 \mathrm{~m}$ or $20 \mathrm{~m}$ at $10 \mathrm{~Hz}$ or $7.5 \mathrm{~Hz}$ in frame suppression mode respectively. With about a $1.5 \mathrm{~m}$ distance from the sample to the detector, the $15 \mathrm{~m}$ length would put the sample at about $13.5 \mathrm{~m}$ which is incompatible with an outer bunker wall at $13 \mathrm{~m}$ and in any case is too close to provide adequate space at the sample for reasonable access and to support complex sample environments. At $20 \mathrm{~m}$, operating in frame suppression mode, QIKR will collect data in as few as 1 to 10 source pulses and it is far more important to collect data across the full required Q-range than to measure an incomplete data set in 30\% less time. Figure 2.6 illustrates the power of QIKR at $20 \mathrm{~m}$ as compared to the current FTS $15.1 \mathrm{~m}$ long Liquids Reflectometer. A second special case is that presented by a traditional SANS geometry where the lowest Q is determined by the lowest scattering angle detector pixel and the longest wavelength used. For a conventional, general purpose SANS as described in Section 3.6, the $7.5 \mathrm{~Hz}$ option allows simultaneous access to $0.0009 \AA^{-1} \leq \mathrm{Q} \leq 1.226 \AA^{-1}$ which will support kinetic 
studies on this instrument. The simultaneous small- and wide-angle scattering instrument, SWANS, described in Section 3.5 is designed to extend this capability to much higher Q, by incorporating a backscattering detector.

Table 2.5 lists 19 STS instrument concepts that were developed in response to a series of workshops that explored the impact and need for neutrons in the next decade and beyond[9] and discussed at an October, 2015 workshop with 200 participants from the neutron user community [10]. Workshop participants identified 12 high-priority instruments identified with ${ }^{*}$ in the table. Also listed in the table are the instrument lengths and the associated bandwidths at the indicated source frequencies. In general, longer instruments selected a length primarily based on satisfying the required wavelength resolution. Shorter instruments tend to choose a length based on the required wavelength band. A mini-workshop held internally at ORNL on $12 / 16 / 2016$ discussed the instrument requirements and came to the following general conclusions which favored choosing an STS frequency greater than $10 \mathrm{~Hz}$ :

1. The reflectometer working group identified the need for QIKR to operate at 7.5 Hz to support its kinetics science mission and provide more working space for GISANS. $20 \mathrm{~Hz}$ was favored for a polarized reflectometer due to uncertainty

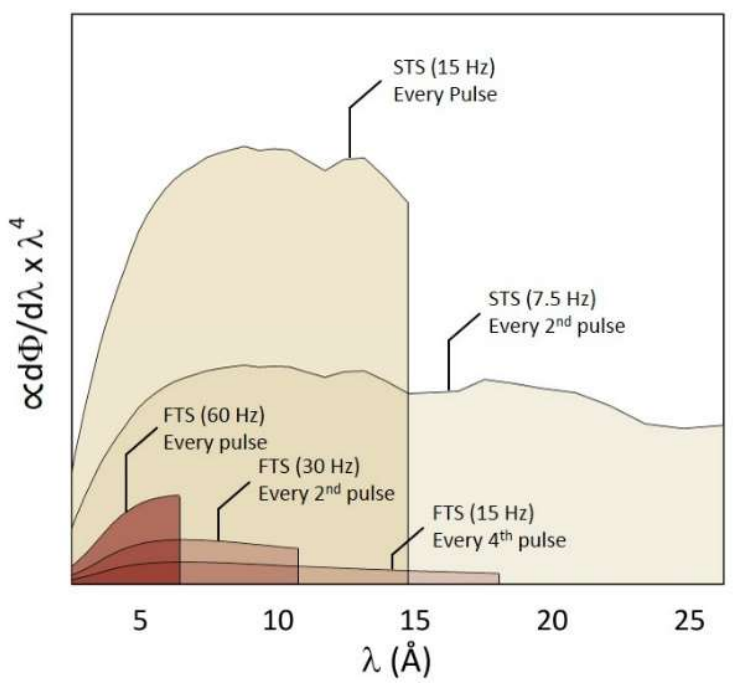

Figure 2.6. Neutron flux multiplied by $\lambda^{4}$ to represent the value of longer wavelength neutrons to a reflectometry measurement. The STS

illustration is for the $20 \mathrm{~m}$ QIKR, while the FTS illustration is for the $15.1 \mathrm{~m}$ Liquids Reflectometer. It should be noted, that it is not possible to actually support $15 \mathrm{~Hz}$ operation on FTS for an instrument this short and the curve is for illustration only. in the ability to effectively enable polarization analysis across a broad wavelength band without significant loss of intensity. Nonetheless, the working group concluded that QIKR benefits more by choosing $15 \mathrm{~Hz}(7.5 \mathrm{~Hz}$ in frame suppression mode) than the quantum condensed matter community who would use a polarized reflectometer benefit from $20 \mathrm{~Hz}$.

2. The diffractometer working group preferred the option of operating at higher frequency and using neutron choppers to suppress frames when experiments required increased band width. The working group was cognizant of the favorable impact of a $15 \mathrm{~Hz}$ choice on the performance of FTS instruments and because of this concluded that $15 \mathrm{~Hz}$ would still give flexibility to exchange bandwidth for flux while preserving the performance of FTS instruments operating in frame suppression mode.

3. The SANS working group preferred $20 \mathrm{~Hz}$ as it provides the highest flux and gave more flexibility. They estimated that the Q-range sampled at the higher frequency was generally adequate.

4. The spectrometer working group recognized that the direct geometry instruments CHESS and HERTZ preferred the highest frequency even with consideration of the benefits of repetition rate multiplication, as the lowest incident energies enabled by lower frequency required too high a fraction of the available counting time relative to the additional information they provided. The hybrid spectrometer JANUS preferred $15 \mathrm{~Hz}$ while the indirect geometry spectrometer BWAVES preferred $10 \mathrm{~Hz}$. BWAVES requires a very large bandwidth of about $23 \AA$ for its science mission and at $10 \mathrm{~Hz}$ 
is already the shortest instrument proposed for STS. The instrument team was concerned that frame suppression would not provide low enough background.

Table 2.5. Instruments identified for STS beam lines with identified lengths and corresponding bandwidths at the various operating frequencies.

\begin{tabular}{|c|c|c|c|c|c|c|}
\hline & & & \multicolumn{4}{|c|}{ Effective Instrument Frequency } \\
\hline & & & $7.5 \mathrm{~Hz}$ & $10 \mathrm{~Hz}$ & $15 \mathrm{~Hz}$ & $20 \mathrm{~Hz}$ \\
\hline \multirow{2}{*}{ Instrument } & $\mathbf{L}(\mathbf{m})$ & $\begin{array}{l}\text { Mod. } \\
\text { Type }\end{array}$ & $\begin{array}{l}\Delta \lambda \\
(\AA)\end{array}$ & $\begin{array}{l}\Delta \lambda \\
(\AA)\end{array}$ & $\begin{array}{l}\Delta \lambda \\
(\AA)\end{array}$ & $\begin{array}{l}\Delta \lambda \\
(\AA)\end{array}$ \\
\hline & \multicolumn{6}{|c|}{ Diffractometers } \\
\hline *VERDI & 50 & $\mathrm{c}-\mathrm{pH}_{2}$ & 10.5 & 7.9 & 5.3 & 4.0 \\
\hline NeSCry & 40 & $\mathrm{c}-\mathrm{pH}_{2}$ & 13.2 & 9.9 & 6.6 & 4.9 \\
\hline *MENUS & 77 & $\mathrm{c}-\mathrm{pH}_{2}$ & 6.8 & 5.1 & 3.4 & 2.6 \\
\hline${ }^{*}$ EWALD & 90.45 & $\mathrm{c}-\mathrm{pH}_{2}$ & 5.8 & 4.4 & 2.9 & 2.2 \\
\hline *HighResPD & 104 & dc- $\mathrm{pH}_{2}$ & 5.1 & 3.8 & 2.5 & 1.9 \\
\hline \multicolumn{7}{|l|}{ Spectrometers } \\
\hline SPHINX & 42 & $\mathrm{dc}-\mathrm{H}_{2} \mathrm{O}$ & 12.6 & 9.4 & 6.3 & 4.7 \\
\hline XTREME-X & 52 & $\mathrm{dc}-\mathrm{H}_{2} \mathrm{O}$ & 10.1 & 7.6 & 5.1 & 3.8 \\
\hline${ }^{*}$ JANUS & 22 & $\mathrm{dc}-\mathrm{H}_{2} \mathrm{O}$ & 24.0 & 18.0 & 12.0 & 9.0 \\
\hline *BWAVES & 16 & $\mathrm{c}-\mathrm{pH}_{2}$ & 33.0 & 24.7 & 16.5 & 12.4 \\
\hline HERTZ & 39 & $\mathrm{c}-\mathrm{pH}_{2}$ & 13.5 & 10.1 & 6.8 & 5.1 \\
\hline${ }^{*}$ CHESS & 29.5 & $\mathrm{c}-\mathrm{pH}_{2}$ & 17.9 & 13.4 & 8.9 & 6.7 \\
\hline${ }^{*}$ MBARS & 75 & dc- $\mathrm{pH}_{2}$ & 7.0 & 5.3 & 3.5 & 2.6 \\
\hline \multicolumn{7}{|l|}{ Multi-Modal } \\
\hline *ZEEMANS & 63.5 & $\mathrm{c}-\mathrm{pH}_{2}$ & 8.3 & 6.2 & 4.2 & 3.1 \\
\hline${ }^{*}$ SWANS & 35.25 & $\mathrm{c}-\mathrm{pH}_{2}$ & 15.0 & 11.2 & 7.5 & 5.6 \\
\hline \multicolumn{7}{|c|}{ Large Scale Structures } \\
\hline${ }^{*} \mathrm{QIKR}$ & 20 & $\mathrm{c}-\mathrm{pH}_{2}$ & 26.4 & 19.8 & 13.2 & 9.9 \\
\hline *VBPR & 36 & $\mathrm{c}-\mathrm{pH}_{2}$ & 14.7 & 11.0 & 7.3 & 5.5 \\
\hline SANS-1 & 35 & $\mathrm{c}-\mathrm{pH}_{2}$ & 15.1 & 11.3 & 7.5 & 5.7 \\
\hline M-WASABI & 37.5 & $\mathrm{c}-\mathrm{pH}_{2}$ & 14.1 & 10.5 & 7.0 & 5.3 \\
\hline SANS-2 & TBD & $\mathrm{c}-\mathrm{pH}_{2}$ & - & - & - & - \\
\hline
\end{tabular}

${ }^{*}$ Instruments identified as high priority at the October, 2015 user workshop.

\subsection{REFERENCES}

[1] Conceptual Design Report Proton Power Upgrade Project, ORNL/TM-2016/672; PPU-P01-PD0001.

[2] Technical Design Report Second Target Station, ORNL/TM-2015/24, January 2015.

[3] Gallmeier, F. X., Moderator Studies for a SNS short-pulse target station, SNS Document: STS03-31TR0004-R00 (2013); Gallmeier, F. X., Lu, W., Riemer, B. W., Zhao, J. K., Herwig, K. W., and Robertson, J. L., Conceptual moderator studies for the Spallation Neutron Source short-pulse second target station, Rev. Sci. Instrum. 87, 063304 (2016).

[4] Review of ORNL Second Target Station Target Design Alternatives, September 22-23, 2015, STS0331-AC0001, R00 (2015). 
[5] STS03-31-AC0002, R00 (2016).

[6] Estia a focusing reflectometer for small samples based on the Selene guide concept, https://www.psi.ch/lns/EstiaEN/estia.pdf.

[7] Early Scientific Opportunities at the Second Target Station, DRAFT.

[8] Zhao, J. K. Gao, C. Y., and Liu, D., The extended Q-range small-angle neutron scattering diffractometer at the SNS, J. Appl. Cryst. 43, 1068-1077 (2010).

[9] Instruments for Emerging Science: A Science Case for the Second Target Station, ed. K. W. Herwig and D. A. Tennant, Neutron Sciences Directorate, ORNL, 2014 (unpublished)

[10] Second Target Station Workshop, Co-chairs: M. R. Eskildsen (University of Notre Dame) and B. Khaykovich (Massachusetts Institute of Technology), https://public.ornl.gov/conferences/neutrons/STS2015/index.shtml. 


\section{PROPOSED STS INSTRUMENT CONCEPTS}

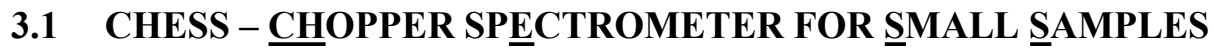

\subsubsection{Executive Summary}

CHESS is a new, direct geometry inelastic spectrometer, which will take full advantage of the increased peak brilliance of the high brightness STS coupled moderators and of recent advances in instrument design and technology to achieve unprecedented performance for inelastic scattering in the cold energy range. For small samples the performance (count rate on the detector array) will exceed that of CNCS by a factor of $\sim 200$.

\subsubsection{Scientific Use}

The wide bandwidth used by the instrument (see Technical Description below) means that it will naturally lend itself to performing exploration-type experiments in which dynamical processes in a material are simultaneously studied in a wide energy range. Therefore, in many cases a study at CHESS will be the first dynamics investigation for a new material by means of scattering. Together with the unprecedented neutron flux on sample, this puts CHESS in a position to conduct experiments with high scientific impact. CHESS will be used to study dynamical processes in hard and soft condensed matter in the $10 \mu \mathrm{eV}-100$ meV energy range.

\subsubsection{Hard Condensed Matter}

CHESS will enable access to four-dimensional reciprocal space to study coherent excitations and dynamic correlations in new phases of strongly correlated, geometrically frustrated, low-dimensional or topological materials, and systems near quantum critical points under extreme conditions with respect to temperature, magnetic (or electric field) and pressure. The instrument will enable studies of how functional materials work, for example, the scattering of heat carrying phonons in thermoelectric materials, the relation between different co-existing order parameters in multiferroics, and ionic transport in battery materials.

CHESS will offer neutron polarization analysis which is beneficial when magnetic phenomena are involved, as it allows the unambiguous separation of magnetic scattering (spin waves) from nuclear scattering (phonons). To date, inelastic scattering with polarized neutrons is better developed for triple axis spectrometers, but the increasing complexity of materials means that more of reciprocal space needs to be covered simultaneously to gain full understanding of a material, which favors time-of-flight methods coupled to large detector areas.

CHESS will push the feasibility limits for sample size/mass for inelastic scattering towards the $\mathrm{mm}^{3} / \mathrm{mg}$ range. This will benefit systems such as flux-grown crystals that are not available in larger size, or systems that are made of precious and rare materials (isotopic enrichment). It will also enable studies of materials at higher applied pressure, $p \sim 10 \mathrm{GPa}$, than previously possible. CHESS will complement diffraction instruments at SNS which are dedicated to small samples, such as SNAP, MANDI and TOPAZ. CHESS will also open the door to studying epitaxially grown materials in which one dimension is reduced to $\sim$ microns. Also coming into view are inelastic measurements of heterostructures where interface effects dominate. Exploration of spin and lattice dynamics in such systems could help to understand their unique magnetic properties and how they differ from the bulk.

Inelastic neutron scattering is the method of choice to probe an excitation spectrum and eigenstates of a system. Usually, selection rules preclude access to all but a few excited states from the ground state. 
Pump-probe experiments allow one to select a specific excited state as the initial state (pulses of microwave radiation, heat, ultrasound, magnetic or electric field) before probing excitations from that state with a synchronized pulse of neutrons. Through the time dependence of the scattering cross section, such pump probe experiments provide unique information including excited state lifetimes and decay pathways that is important for applications in quantum computation and inaccessible through other techniques.

\subsubsection{Soft Matter}

By their very nature, soft materials often host dynamic processes that span the time range from picoseconds to hours or even more. A wide bandwidth instrument such as CHESS can probe a significant fraction of these processes simultaneously.

The bulk dynamics of polymers, proteins with hydration water, gels, colloids and such systems has long been a major application of high resolution neutron spectroscopy. It will now be possible to move to systems with reduced dimensions such as membranes, and the interaction of proteins through membranes. Biological function of proteins is intimately coupled to conformational changes and internal dynamics, and the increased sensitivity of CHESS can make far reaching contributions to our understanding of bioand bio-inspired materials.

The polarized beam at CHESS may also be of interest to this community. Polarized neutrons allow one to separate coherent and incoherent scattering processes in hydrogenous materials, which correspond to collective and single-molecule dynamics, respectively. Most of this type of research is being performed to-date in the quasi-elastic scattering regime with spin-echo methods, because of the limitation to instruments with small detectors.

\subsubsection{Technical Description}

At the present time, the instrument design is still conceptual. While some of the design choices are becoming firm, no final decisions have been taken and the optics design has not been finalized yet. Therefore, performance estimates are approximate. However, engineering reality is already part of the conceptual design, that is, chopper positions avoid the bunker wall, appropriate gaps have been foreseen in the guide to host choppers, the footprint takes into account neighboring instruments, and so on.

\subsubsection{Engineering Layout}

The main scientific goal, to measure small samples with medium to coarse energy resolution, has three main consequences for the technical instrument design.

- The total length to the detector should be $\sim 32$ meters. At this length, the contribution of the source pulse width to the energy resolution is estimated to be $\Delta E / E_{\mathrm{i}} \sim 0.029$, for intermediate energy, $\sim 5 \mathrm{meV}$, using a moderator that is optimized for integral brightness [1]. With the other two contributions to the resolution (from the $M$ chopper and from the time uncertainty between sample and detector) adding in quadrature, the coarse resolution limit (effectively running without $P$ chopper) in matched condition will be $\Delta E / E_{\mathrm{i}} \sim 0.029^{*} \operatorname{sqrt}(3) \sim 0.05$. Matching the three contributions to the resolution ensures maximum flux for resolution. The short overall length implies a wide bandwidth.

- The design of the detector tank will have to maximize detector coverage. It will be desirable to place detector tubes all around in the scattering plane (like it is traditionally done), but also above and below the plane. A short sample to detector distance (current design is 2.5 meters) is acceptable. There may not be room for a gate valve to isolate the sample area - a detailed engineering study will 
be required - and thus the tank may be filled with inert gas during operation (this is an open question) to minimize equipment change-out times.

- The beam at the sample must be small (to match sample size) but divergent (for intensity). Therefore the guide must come close to the sample, to about $\sim 10 \mathrm{~cm}$. Beam polarization must occur upstream. The workhorse cryostat must have a thin tail. A split-coil cryomagnet must incorporate a guide section in its body.

Figure 3.1 below shows the current conceptual engineering layout.
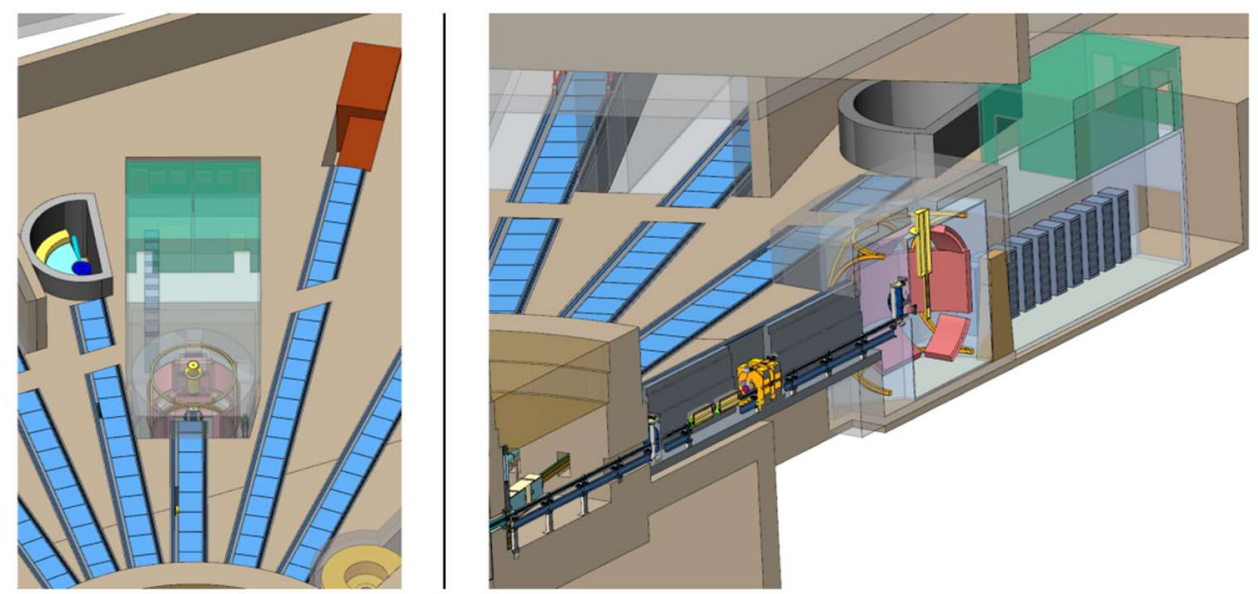

Figure 3.1. Conceptual engineering layout for CHESS that shows how the instrument will fit in its environment in the facility. The beam line will be straight, $\sim 30$ meters to the sample, with one chopper inside the front bunker. Access to the sample will be from the top. The tank for the secondary spectrometer will be in a block house but mostly underground and will also host large coils for xyz polarization analysis and magnetic shielding. User areas will be on the back side.

\subsubsection{Functional Layout (Guides and Choppers)}

Figure 3.2 below shows a conceptual functional layout that shows the neutron guide (including some transported neutron trajectories), chopper locations, double-V-shaped transmission polarizer, and a timedistance diagram with 6 accepted sub-pulses per STS frame. Enough time passes between pulses such that energy transfers up to $\sim 80 \%$ can be observed without frame overlap. The anticipated chopper system allows for much flexibility regarding the number of pulses used and the allowed energy transfer between them.

The beam line is straight. The flash pulse (neutrons and photons) will be blocked by a $T_{0}$ chopper that is located at half the distance between source and detector. In this location it will only make one "shadow" (blocking good neutrons) on the detector. It is expected that this chopper will reduce the extent of the unusable range on the time axis to less than $2 \mathrm{~ms}$ (this is a conservative estimate). 


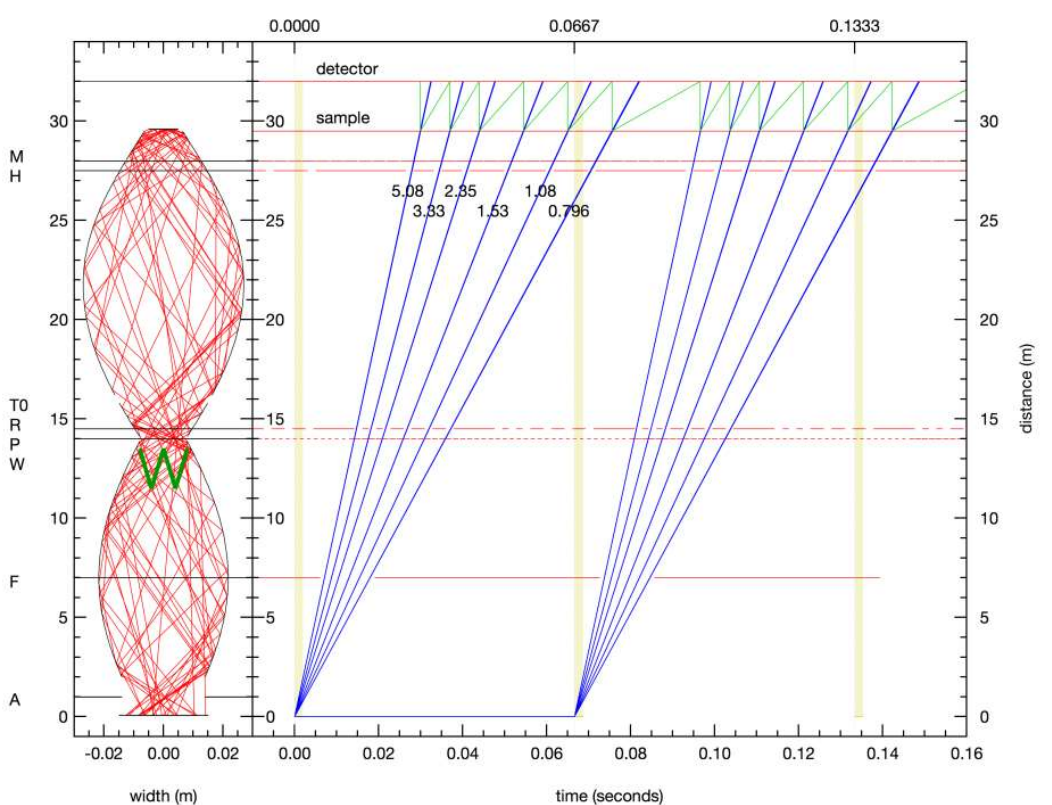

Figure 3.2. Left: Two ballistic sections of neutron guide transport the beam from the source (bottom) to the sample (top). The guide starts at 2 meters distance from the source. An initial aperture $(A)$ of fixed size controls the divergence going into the guide. Choppers following in order are: Frame Overlap $(F)$, Pulse Shaping $(P)$, Pulse Removal $(R)$, Prompt-Pulse Blocker $\left(T_{0}\right)$, Hand $(H)$ and Monochromating $(M)$ chopper, respectively. All except the $T_{0}$ chopper are double disk choppers. The biggest gaps are needed for the $T_{0}$ chopper $(40 \mathrm{~cm})$ and the $P$ chopper $(24 \mathrm{~cm})$, respectively. $(W)$ denotes a double- $\mathrm{V}$ transmission polarizer. The right hand side shows a time-distance diagram with 6 accepted pulses.

The $P$ and $M$ choppers will be counter-rotating double disk choppers rotating at $300 \mathrm{~Hz}$. These will be phased such that the second disk opens as the first disk closes. In such an arrangement, the effective opening time will be wavelength dependent and will linearly increase with wavelength [2]. This scheme will allow acceptance of longer pulses at longer wavelengths and will make the energy resolution $\Delta E / E_{\mathrm{i}}$ independent of $E_{\mathrm{i}}$. With such a chopper system, the energy resolution is not controlled by the chopper speed (meaning that the $P$ and $M$ choppers will always run at the same speed) but by the disk separation. At the $M$ chopper, the disk separation will vary between $\sim 2-5 \mathrm{~cm}$, at the $P$ chopper between $\sim 4-20 \mathrm{~cm}$. All other choppers are parallel rotating double disk choppers rotating at base frequency. An increase of the design speed of the $P$ and $M$ choppers to $360 \mathrm{~Hz}$ will be investigated later, and would provide a performance gain, but is not considered essential.

The guide consists of two main sections of ballistic shape. Making the guide narrow around the $P$ chopper involves some losses in neutron transport but the chopper will function more effectively. The optimal beam width in this location remains to be established. The guide coating will make use of $m \sim 2.5-3.5$ supermirrors. Since the guide is narrow, the internal reflection angles will not be very large and higher $m$ values are not required. Nevertheless, in the target operation range the brilliance transfer is expected to be $70 \%$ and higher, see Figure 3.3. In the near future, options will be explored to increase the available divergence on the sample, perhaps up to $\pm 3 \mathrm{deg}$. While such a large divergence will rarely ever be used (the beam can always be tailored with apertures such as at the WISH instrument [3]), it will be desirable to increase the divergence for selected samples (very small samples, 1D- or 2D-dimensional systems) to 
more than \pm 1 deg. Given the large divergence and small size of the beam at the sample, it is expected that the guide approaches the sample to within $\sim 10 \mathrm{~cm}$.

The double-V-shaped $m=5$ transmission polarizer is situated at the end of guide section 1 where the beam is relatively narrow. It can be removed from the beam line with a translation table or barrel arrangement. It will polarize neutrons in a $\sim 2-12 \AA$ band simultaneously. A permanent guide field will maintain the neutron polarization from $\sim 13$ meters out to the sample. To analyze the scattered polarization, ${ }^{3} \mathrm{He}$ filter cells and solid state transmission analyzer mirror arrays will be considered at a later time.
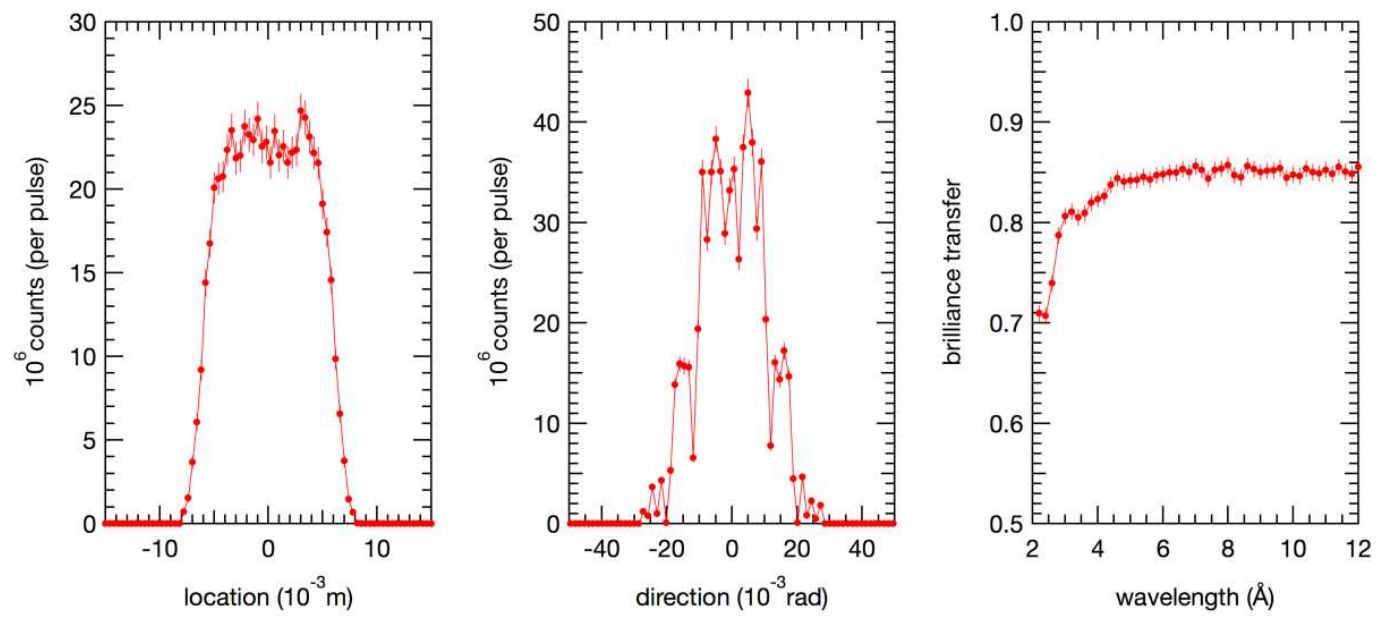

Figure 3.3. Preliminary neutron transport calculation. Beam on sample is shown. White beam intensity (2-12 $\AA$ ) is $\sim 7 \times 10^{8}$ per pulse at $500 \mathrm{~kW}$. The presented guide will put a beam on sample that is slightly more than $1 \mathrm{~cm}$ wide with a full divergence of $\pm 1 \mathrm{deg}$. The brilliance transfer converges to $\sim 85 \%$ at long wavelength (this represents the losses in the various gaps). Additional losses at short wavelength are due to imperfect reflections in the guide.

\subsubsection{Other Components}

\section{Shutters}

The facility will provide a maintenance shutter in the bunker that generally will only be closed during facility shut-down periods. Each beam line will have one or more operations shutters which block thermal and cold neutrons and are utilized for daily experimental operations (i.e., sample changes). The maintenance shutter moves between three positions, open, neutrons blocked, gamma radiation blocked. This shutter will nominally be in the open position during the entire operational period. The "neutrons blocked" position provides a means to allow activated downstream components to decay while the source is operating but will not allow access to beam line equipment that is between the two shutters. The final position of the maintenance shutter will be used when the source is off to allow access to all downstream equipment. Together the maintenance and operations shutters will ensure access to the secondary spectrometer for sample changes during operation and the reduction of secondary activation of components during operation if necessary. 


\section{Detector Tank}

The detector tank will host the secondary spectrometer which includes the detector array, some of the associated electronics, collimation, xyz coils for a magnetic guide field, the magnetic shielding for these coils, and sample environment equipment. The desire to maximize detector coverage may make it impossible to include a thimble/gate valve near the sample position (this is an open question that needs to be evaluated from the engineering side). The requirement to minimize equipment change times couples this question to the choice whether the tank will be evacuated or argon-gas filled during operation.

\section{Detector Array}

The primary choice to date would be to use $1 / 2$-inch ${ }^{3} \mathrm{He}$ tubes with $\sim 6$ bar pressure. The length of the tubes would be $\sim 1.5-2$ meters. At $\sim 2.5$ meters distance to the sample it would take $\sim 3,300$ tubes to cover $2 \pi$ and would need $\sim 5,000$ liters of ${ }^{3} \mathrm{He}$ would be needed with 6 bar. Bringing the detectors closer to the sample would save cost but would have three main adverse effects: frame overlap would be tighter (meaning that one would have fewer pulses to count, this is a proportional intensity loss), one would have to shorten the pulse at the $M$ chopper for the same resolution (this is also a proportional intensity loss), and one would lose $\mathrm{Q}$ resolution (mostly at low angles). Technologies based on ${ }^{10} \mathrm{~B}$ capture in solid thin sheets or tubes are an emerging alternative to consider. In the cold neutron range such detectors are already competitive today.

\section{Collimator}

Collimation between sample and detectors is absolutely essential for the proper functioning of the instrument. The current plans for this call for 3D printed collimators which are already in use at the VISION instrument at SNS but which need further development.

\section{Fully Integrated Sample Environment}

The beam line has very particular requirements for sample environment equipment some of which are mentioned above. It is currently anticipated that all such equipment is fully optimized for CHESS and likely not shared with other beam lines. The workhorse equipment will be a thin tail cryostat with ${ }^{3} \mathrm{He}$ insert and dilution insert, and a cryomagnet similar in dimension to the 5T magnet the SNS currently operates at CNCS, HYSPEC and CORELLI.

\section{Slits}

Slits will be included in the guide in strategic locations to limit beam size and beam divergence (independently in the horizontal and vertical directions) as needed for each particular experiment.

\section{Monitors}

It is essential to monitor the beam behind the high-speed choppers and in other locations. A shortcoming of traditional monitors in use at CNCS and elsewhere is that their efficiency cannot be made low enough. Therefore, in lieu of such beam monitors, a thin vanadium foil (or another suitable scatterer) may be placed in the beam at such locations, and the scattered beam may be picked up by a conventional ${ }^{3} \mathrm{He}$ tube located next to the beam line. 


\section{Beam Stop and Get-Lost Tube}

It is currently foreseen to incorporate the beam stop in the sample environment as it is done at CNCS, and to leave out a get-lost tube. This concept is now awaiting a high energy neutron transport analysis.

\subsubsection{Impact of STS Operating Frequency}

To first order, a direct geometry inelastic spectrometer at a spallation source will see a linear performance increase with increased source frequency until a limit is reached and the performance becomes flat. At the limit, the time elapsed between pulses corresponds to the time needed to collect neutrons with energy loss, and thus the optimal frequency depends on the working incident energy, $E_{\mathrm{i}}$. Beyond the limit (at higher frequency) one would use a pulse removal chopper. This of course assumes that the accelerator and target systems can deliver the same energy per pulse at higher frequency. A one-pulse mode of operation is the most flexible mode. Put in perspective, the optimum frequency for CNCS is $\sim 120 \mathrm{~Hz}$ and for ARCS $\sim 300 \mathrm{~Hz}$ (if not higher). For CHESS this means that $20 \mathrm{~Hz}$ would be preferred over $15 \mathrm{~Hz}$, with possibly $\sim 33 \%$ better performance, depending on the relative value of the lowest incident energy sub pulse to the science.

However, a lower repetition rate minimizes the number of flash pulses generated which create "dead zones" on the time axis where thermal or cold neutron cannot be counted. This is a consideration for CHESS as it will not be limited to counting only in the first frame.

\subsubsection{Technical Maturity}

ORNL has built a spallation neutron source previously and has learned valuable lessons during that project. Much of the in-house expertise gained at the time is still present at ORNL.

The main components, choppers, guides, detectors, tank, shielding, are established technology and present a known, and comparatively little, risk. A chopper speed of $300 \mathrm{~Hz}$ will be sufficient to deliver the science. The guide design does not call for particularly high $m$-coating.

The main risk at this point may be the 3D-printed collimator. Small prototypes pieces are available but the full scale is beyond what has been done to-date.

The polarization analyzer device is another big risk. The detector array may be close enough to make a supermirror device feasible (such as at HYSPEC or D7 at ILL). ${ }^{3} \mathrm{He}$ analyzer cells are an alternative with the potential for improved performance. Conceptually the main difference is that a mirror device would be a permanent installation (covering about half of the in-plane detectors) whereas ${ }^{3} \mathrm{He}$ cells would be inserted as needed.

The optics alignment needs to meet tolerance and precision requirements that are significantly tighter than on the first target station. However, current survey and alignment technology should be able to handle the requirements.

\subsubsection{References}

[1] F. X. Gallmeier, W. Lu, B. W. Riemer, J. K. Zhao, K. W. Herwig, and J. L. Robertson, "Conceptual moderator studies for the Spallation Neutron Source short-pulse second target station", Rev. Sci. Instr. 87063304 (2016). 
[2] A. Vickery and P. P. Deen, "Choppers to optimise the repetition rate multiplication technique on a direct geometry neutron chopper spectrometer”, Rev. Sci. Instr. 85115103 (2014).

[3] Laurent C. Chapon, Pascal Manuel, Paolo G. Radaelli, Chris Benson, Leigh Perrott, Stuart Ansell, Nigel J. Rhodes, Davide Raspino, D. Duxbury, E. Spill, and Julian Norris "Wish: The New Powder and Single Crystal Magnetic Diffractometer on the Second Target Station" Neutron News 2 22-25 (2011). 


\subsection{ZEEMANS}

\subsubsection{Executive Summary}

ZEEMANS is a multi-modal instrument built around a dedicated high-field vertical-bore split-coil magnet with maximum steady-state field-at-sample of 35 Tesla. It will take full advantage of the increased peak brilliance of its high brightness STS coupled moderator, and of recent advances in instrument design and technology.

A magnet capable of producing 35 Tesla field requires infrastructure that prevents a conventional strategy of moving the magnet between beamlines to exploit different scattering techniques. Instead, a flexible chopper system, a configurable pre-magnet optics section, and modular back-end flight paths and detectors (referred to hereafter as 'spokes') will enable a variety of scattering techniques.

The initial implementation will enable multiplexed indirect geometry spectroscopy and single crystal diffraction. The instrument will be designed to allow for expansion of capabilities in the future, providing opportunities for small angle neutron scattering, reflectometry, and direct geometry spectroscopy together with the incorporation of polarization analysis.

\subsubsection{Scientific Use}

Access to magnetic fields of up to 35 Tesla would significantly broaden the steady-state magnetic-field range, and would allow access to new problems that can be addressed with neutron scattering. Applied magnetic field is a continuously adjustable, directional, thermodynamic tuning parameter that affects materials by raising and lowering the spin energies of the outer-shell valence electrons, exerting torques on the orbital and spin magnetic moments, controlling quantum fluctuations, and ultimately driving phase transitions. Experiments that explore materials in extremely high magnetic fields provide clear evidence of exciting new phenomena, but to understand the structure and dynamics of these new phases, neutron scattering experiments under high-field conditions are essential.

The Quantum Materials section of the "Early Scientific Opportunities at Second Target Station" [1] highlights many examples where both inelastic and elastic neutron scattering from materials will make a strong impact by utilizing the expanded field range provided by ZEEMANS. Examples include:

- The quantum spin liquid is an example of a quantum disordered state in which magnetic fields can suppress quantum fluctuations, allowing the influence of such fluctuations to be continuously tuned. In the fully polarized state, inelastic neutron scattering can reveal the bare, underlying interactions that cannot be extracted from other experimental techniques. This provides critical information to understand the entangled nature of the quantum spin liquid.

- Topological spin structures such as skyrmions are predicted to exist in chiral phases in a variety of materials. These phases are stabilized in magnetic fields, but some predicted skyrmion states may only exist at magnetic fields currently inaccessible to neutron scattering. High resolution diffraction in the presence of such fields will enable detailed exploration of these incommensurate states that is critical to understand the underlying topology.

- High magnetic fields can be used to suppress superconductivity, which can reveal the nature of a potential quantum critical point beneath the cuprate superconducting dome. Fields provided by ZEEMANS can suppress superconductivity in some cuprates, and both elastic and inelastic scattering can reveal the nature of the underlying magnetism, shedding light on the fundamental properties of the quantum critical point that apparently drives superconductivity. 
- Fields of $\sim 35$ Tesla can suppress the "hidden order" phase in $\mathrm{URu}_{2} \mathrm{Si}_{2}$ and severely perturb or access quantum criticality in other heavy fermion systems, including $\mathrm{SmB}_{6}, \mathrm{CeRh}_{2} \mathrm{Si}_{2}$ and $\mathrm{CeCu}_{6}$. By breaking up such strongly correlated systems with an applied field while allowing for elastic and inelastic neutron scattering, there is the potential for major breakthroughs in our understanding of such systems.

- Frustrated magnets often exhibit complex phase diagrams in the presence of applied magnetic fields. Diffraction on ZEEMANS will reveal underlying structures at high magnetic fields in a variety of frustrated magnetic materials, helping to understand the competing interactions of these complex, magnetic materials.

- High magnetic fields can induce a Bose Einstein Condensation of magnons in certain lowdimensional magnetic systems. Some of these phases require fields greater than $30 \mathrm{~T}$ and measurements of the excitation spectrum and field-induced ordered state can provide valuable information needed to understand this phenomenon in a wide variety of materials.

A 35 Tesla magnetic field imposes a natural energy scale that must be matched to the instrument design. The Zeeman energy is $g \mu_{\mathrm{B}} \mathrm{S} \cdot \mathrm{B}$; for a simple $\mathrm{S}=1 / 2$ system, one can take $\mathrm{g}=2$ and $\mathrm{S}=1 / 2$ which with $\mu_{\mathrm{B}}=0.058$ $\mathrm{meV} / \mathrm{T}$ means that a $35 \mathrm{~T}$ magnetic field amounts to $2 \mathrm{meV}$. Consequently, the best scientific impact for ZEEMANS will occur with optimized intensity for cold neutrons and, hence, the minimum incident energy will be $\sim 1 \mathrm{meV}$. However, some problems like the cuprate example described above use magnetic field to suppress specific phases and, hence, the appropriate energy scale is not defined by the magnetic field energy but, rather, the

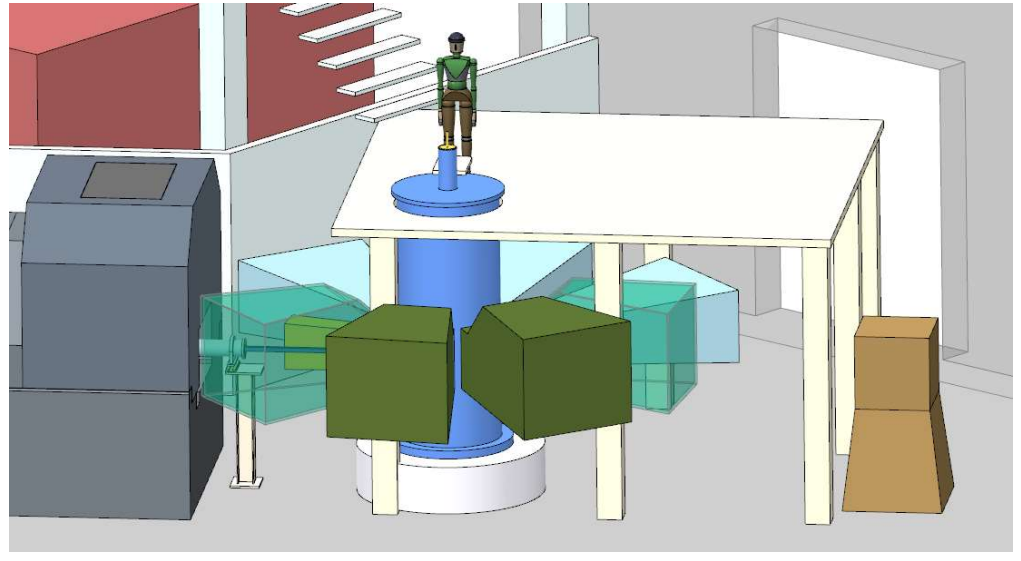

Figure 3.4. Concept of the ZEEMANS instrument. The conceptual engineering layout shown represents 8 equally spaced openings separated by 8 shielding wedges. Initially, half the openings will be multiplexed indirect geometry spectrometers (green) and with diffraction detectors viewing the remaining openings (blue). underlying energetics of the material. In such cases, energies of hundreds of meV would be ideal to explore the dynamics of such problems. Consequently, the scientific case results in a natural range of energies for ZEEMANS of 1-200 meV with particular emphasis on the lower energy portion of the spectrum, where many important scientific problems are emerging.

\subsubsection{Basic Instrument Concept}

The conceptual design is based around a vertical split coil magnet with wedges separating the coils. Half of the instrument will be surrounded by a position-sensitive detector array optimized for diffraction experiments while the remaining half will be surrounded by a secondary spectrometer to enable indirect geometry spectroscopy (IGS), which is the best strategy to cope with the restricted angular view provided by a high field magnet. This spectrometer will be multiplexed to enable broad sampling of $\mathbf{Q}, \omega$ space and will utilize a concept similar to the CAMEA concept being developed for the BiFROST instrument at the European Spallation Source [2]. There are several technical advantages achieved via this multiplexed spectrometer approach as opposed to a direct geometry spectrometer (DGS) approach: an IGS utilizes an incident white beam that allows both diffraction and inelastic measurements to be performed 
simultaneously; an IGS, as opposed to a DGS, does not require a chopper very near the sample which is a technical challenge with such high magnetic fields. These technical considerations, together with the advantages afforded by a large number of final energies on the CAMEA concept, makes this the best choice for the inelastic spectrometer on ZEEMANS.

\subsubsection{Future Scattering Techniques}

As a multi-modal beamline, the instrument will be designed to preserve the option for future scattering techniques based on the scientific needs of the user community. Potential options include SANS, reflectometry, and a DGS. A DGS future option could enable measurements with higher energy resolution and potentially lower background in cases where signals are weak. The initial concept will not provide polarized neutrons, but future addition of polarized capabilities will be scientifically beneficial for certain problems and to enable polarized reflectometry measurements. Designing the magnet with an asymmetric option will enable polarization options in the future.

\subsubsection{Technical Description}

In Table 3.1, basic and common instrument parameters and sample sizes are displayed. The wavelength range is presented for the full desired range, including and up to $200 \mathrm{meV}$. However, for IGS, the main incident energy range of $2.5 \mathrm{meV}$ to $80 \mathrm{meV}$ is consistent with our choice of cold coupled moderator which maximizes flux for cold neutrons.

Different techniques will be optimized for different sample dimensions and incident energy ranges. A precedent exists for such a multi-technique time of flight instrument: EXED at Helmholtz-Zentrum Berlin [3]. However, the horizontal magnet geometry at EXED motivated different priorities and strategies for different techniques. EXED initially offered single crystal diffraction and small angle neutron scattering, and later introduced direct geometry spectroscopy (DGS).

\subsubsection{Magnet Technology}

The EXED instrument at Helmholtz Zentrum Berlin is also the only operational neutron instrument internationally which is optimized around a high field magnet [4]. That magnet employs a resistive / superconducting hybrid design that was the only technology capable of achieving steady-state fields in excess of $\sim 15$ Tesla at that time. The ZEEMANS magnet, however, will exploit recent performance enhancements of commercially available composite conductors that employ high temperature superconductors (HTS), and recent developments for coils and integrated magnet systems that leverage these conductors. HTS coils achieve higher critical current density in the presence of high ambient magnetic fields than is possible using low temperature superconductors (LTS), which in turn enables higher field at sample position. This new magnet technology will be further developed and the magnet constructed by the Florida State University's National High Magnetic Field Laboratory (NHMFL), which is currently commissioning a 32 Tesla user magnet employing insulated rare earth barium copper oxide (ReBCO) [5], and is actively developing a no-insulation ReBCO solenoidal coil technology as shown in Figure 3.5 [6], which includes a clear path

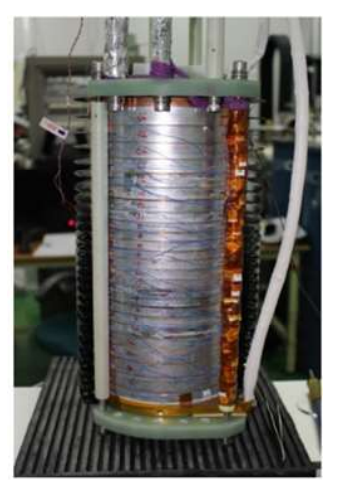

Figure 3.5. Test stack of pancakes coils employing no-insulation ReBCO composite conductor [6]. 
towards a 20 Tesla NI-ReBCO only user magnet. The NHMFL participates in the ZEEMANS instrument advisory team, and ORNL is actively communicating our desired specifications and intends to spur the appropriate technology via appropriate subcontracts with NHMFL as part of the STS project.

The ZEEMANS magnet concept will employ a vertical bore, split coil geometry. This geometry provides easy sample access, sample rotation, and enables a wide range of sample conditions using conventional top-loading inserts and a cold bore. Access to more of the $\pm 180^{\circ}$ scatter range in the plane that is perpendicular to the rotation axis - compared to only the forward and back scattering afforded by a horizontal conical-bore geometry - enables more flexibility to simultaneously optimize momentum transfer range and resolution. The range of angles to be measured will be optimized as part of the magnet design, and a wedge support design is envisaged to accommodate the large forces between coils. One concept for a wedge design in shown in Figure 3.6 and summarized in the Table 3.2. The inelastic spectrometer and diffraction banks will accommodate this optimized wedge design.

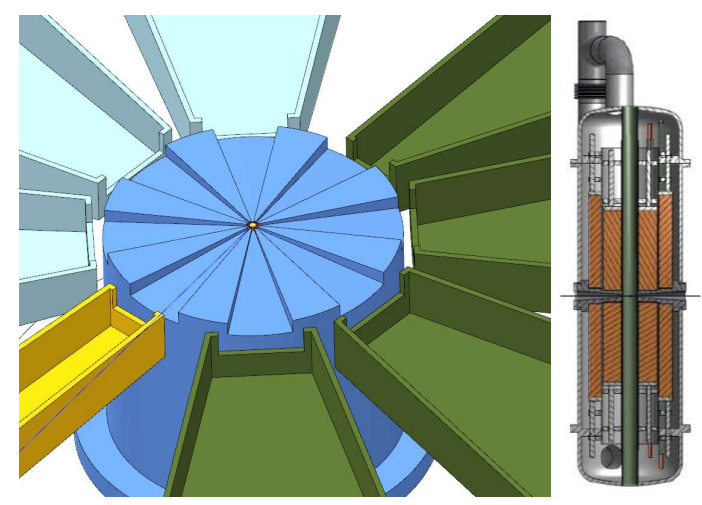

Figure 3.6. Isometric rendering of the magnet design being evaluated, cut at beam elevation, showing the wedge-support geometry of the magnet and access for incident and scattered beams. Eight openings of $25^{\circ}$ horizontal openings are present, providing a total of $200^{\circ}$ coverage in the horizontal plane. Also shown to a different scale is a cross section for a possible magnet design employing no-insulation ReBCO coils.

Table 3.2. ZEEMANS magnet configuration concept.

\begin{tabular}{rcc}
\hline Maximum field & (Tesla) & 35 \\
Spoke Openings & & 8 \\
Horizontal Spoke & $(\mathrm{deg})$ & 25 \\
Angle & & 10 \\
$\begin{array}{r}\text { Vertical Spoke Full } \\
\text { Angle }\end{array}$ & $(\mathrm{deg})$ & $( \pm 5)$ \\
$\begin{array}{r}\text { Inner Vacuum } \\
\text { Chamber Diameter }\end{array}$ & $(\mathrm{mm})$ & 35 \\
\hline
\end{tabular}

\subsubsection{Primary flight path distance and magnetic stray field}

The magnet / sample position will be selected to provide sufficient distance from other instruments to mitigate the effect of ZEEMANS' stray fields. To this end, and to accommodate the substantial support systems, the ZEEMANS instrument will be housed in an external building. Given that constraint, and to avoid the proton beam path components leading to the Second Target Station building, possible positions for the magnet range between $45 \mathrm{~m}$ and $60 \mathrm{~m}$ downstream from the moderator. Most techniques would benefit from a shorter distance, due to increased bandwidth, better matching to energy or momentum transfer resolution of the secondary instrument, and better transmission due to shorter transport, but the 
magnet's stray field will determine if $45 \mathrm{~m}$ is an acceptable distance. At $15 \mathrm{~Hz}$ operation, the wavelength bandwidth at a sample position of $45 \mathrm{~m}$ is about $50 \%$ of the full instrument bandwidth specified, although this varies slightly for techniques with differing flight paths, and at $7.5 \mathrm{~Hz}$ the bandwidth is about $100 \%$. In contrast, for a sample position of $60 \mathrm{~m}$, the bandwidth is only about $35 \%$ and $70 \%$ for $15 \mathrm{~Hz}$ and 7.5 $\mathrm{Hz}$ operation, respectively. Stray field suppression strategies including active compensation and passive shielding will be considered as part of the magnet / instrument design.

\subsubsection{Guides and Choppers}

Bandwidth, frame overlap, and T0 choppers are all planned as part of the common pre-sample instrument mode of operation. Preliminary work has begun to position these choppers (see Figure 3.7). Such choppers and chopper functions are established features for ToF instruments and do not present a technological risk. Several techniques require a chopper a few meters upstream of the magnet, including the CAMEA technique for IGS, and such choppers will be part of a configurable 5 meter section upstream of the magnet. Preliminary work has also begun to define the guide configuration, which we expect to employ a conventional straight ballistic geometry with both horizontal and vertical focus near or at the sample.

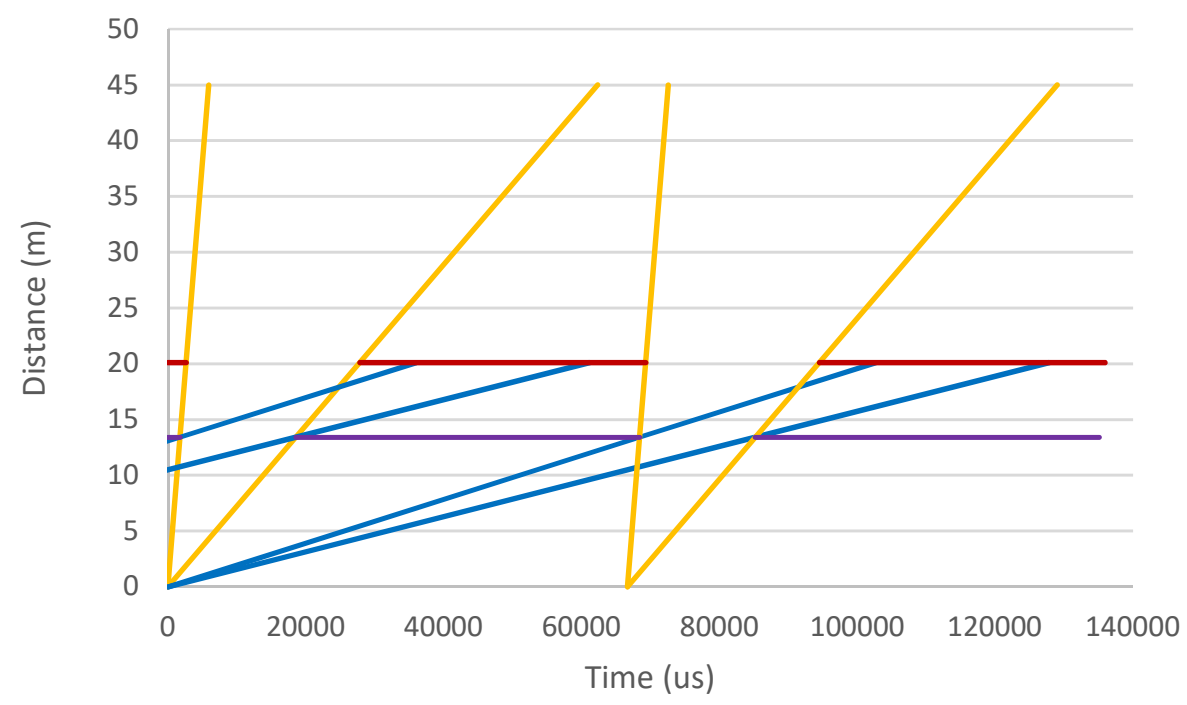

Figure 3.7. Timing diagram for $15 \mathrm{~Hz}$ operation with a source to sample distance of $45 \mathrm{~m}$.

To facilitate current and future scattering applications on ZEEMANS, a modular, reconfigurable guide design is required. The current concept is to use a straight (ballistic) guide until 5 meters before the sample to transmit the full spectrum of incident neutrons. Components common to all scattering techniques are summarized in Table 3.3. In the 5 meters before the sample, the standard configuration will employ elliptical or parabolic focusing with a strategy to reduce the beam divergence for situations where tighter collimation is needed. Various optics components like polarizers, flippers, and choppers can be added in this section to facilitate future scattering applications. 
Table 3.3. Components common to all instrument options

\begin{tabular}{|c|c|c|}
\hline Distance (m) & Component & Motivation / Description \\
\hline $1-5.15$ & Straight Guide & Focus near sample \\
\hline 5.33 & Maintenance Shutter & Closed only during maintenance inside Bunker \\
\hline $6.2 \mathrm{~m}$ & $\begin{array}{c}\text { Frame Overlap } \\
\text { Chopper }\end{array}$ & All techniques. \\
\hline $7.5-10.5$ & Straight Guide & Focus near sample \\
\hline 11.2 & T0 Chopper & $\begin{array}{l}\text { Reasonable transmission at the maximum Ei of } 200 \mathrm{meV} \text { requires a } \\
\text { straight guide and T0 Chopper. } 200 \mathrm{meV} \text { open requires } 5.7 \mathrm{~m} \\
\text { minimum (bunker restriction } 6.2 \mathrm{~m} \text { ). Prefer inside bunker to limit } \\
\text { secondary scatter, so at or less than } 11.2 \mathrm{~m} \text {. Reflectometry and SANS } \\
\text { benefit from extended bandwidth of } 7.5 \mathrm{~Hz} \text {, so to limit occlusion we } \\
\text { prefer a maximum distance from the moderator. }\end{array}$ \\
\hline 12.2 & Operational Shutter & Closed for magnet $\&$ instrument access. Inside the outer bunker wall. \\
\hline $\begin{array}{l}13.2 \text { - modular } \\
\text { pre-sample }\end{array}$ & Straight Guide & Focus near sample \\
\hline 13.4 & $\begin{array}{l}\text { 2nd Frame Overlap } \\
\text { Chopper }\end{array}$ & All techniques. \\
\hline $\begin{array}{l}14 \text { - modular } \\
\text { pre-sample }\end{array}$ & Neutron Monitors & $\begin{array}{l}\text { Strategically placed to measure against chopper performance, with } \\
\text { sufficient count rate to obtain statistically significant wavelength } \\
\text { dependent counts per pulse. }\end{array}$ \\
\hline 20.1 & Bandwidth Chopper & All techniques. \\
\hline
\end{tabular}

\subsubsection{Components common to all scattering techniques}

The following components will be in place for use with all scattering techniques; distances are approximate.

\subsubsection{Diffraction and Multiplexed Indirect Geometry Spectroscopy Configurations}

The diffraction portion of ZEEMANS will consist of ${ }^{3} \mathrm{He}$ linear position sensitive detector tubes. Each diffraction spoke will employ 20 8-packs of $8 \mathrm{~mm}$ diameter tubes. The distance from the sample to these diffraction detectors is 3.5 meters.

The initial concept for the IGS design will follow closely the approach taken with the CAMEA spectrometer being proposed for the BiFROST instrument at ESS [2,7]. This concept employs an array of 10 back-to-back PG Rowland focusing arrays of PG (002) analyzer crystals each optimized to scatter at different final energies. Relatively long distances are used between sample and analyzer, and analyzer to detector to ensure resolution is determined by geometry instead of analyzer mosaic, and to leverage the mosaic to enable each focusing array to direct different scattered energies to different adjacent detectors. Long and specific distances are also required to work with an order sorting chopper located before the sample which enables separation of first and second diffraction orders at different detection time windows. The main difference between our system and the CAMEA system is that CAMEA is restricting itself to $\pm 1.4^{\circ}$ whereas the ZEEMANS magnet as envisioned has an available vertical divergence of $\pm 5^{\circ}$; for initial performance estimates, we assume the IGS vertical divergence is limited to $\pm 1.4^{\circ}$. 


\subsubsection{Performance}

We estimate the flux on sample as follows. Figure 3.8 shows the ZEEMANS moderator brightness. At 45 $\mathrm{m}$ distance, the bandwidth is $5.86 \AA$. The integral under the curve is $1.2 \mathrm{e} 13$, taken from $1 \AA$ to $6.86 \AA$. A reasonable estimate for an entrance aperture is that it presents $2 \mathrm{e}-4 \mathrm{sr}$ solid angle. For example, a $3 \mathrm{~cm}$ circular aperture at 2 meters would be of that size. Assuming 50\% transport to the sample, the white beam integrated intensity in the band from $1 \AA$ to $6.86 \AA$ is then $1.2 \mathrm{e} 9 \mathrm{n} / \mathrm{cm}^{2} / \mathrm{s}$.

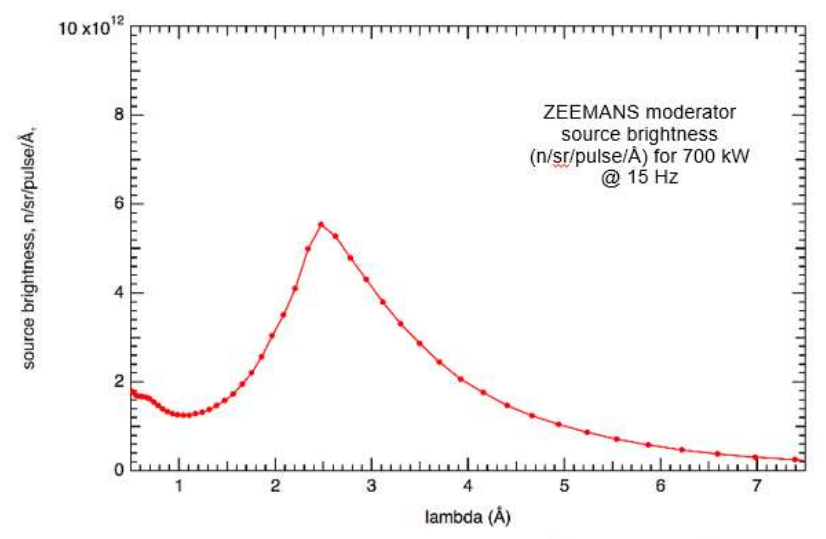

Figure 3.8. Moderator brightness (n/sr/pulse/Å) for the $5 \mathrm{~cm}$ x $5 \mathrm{~cm}$ coupled cold moderator for STS.

\subsubsection{Indirect Geometry Spectroscopy Performance}

Vertically divergent $\left( \pm 1.4^{\circ}\right)$ scattering is captured via Rowland focused pyrolytic graphite crystals onto ${ }^{3}$ He LPSD's, so vertical resolution is sacrificed for increased count rate. Following [8], we calculate the incident energy resolution, and following [7] we obtain the final energy resolution, and display the results in Figure 3.9. 

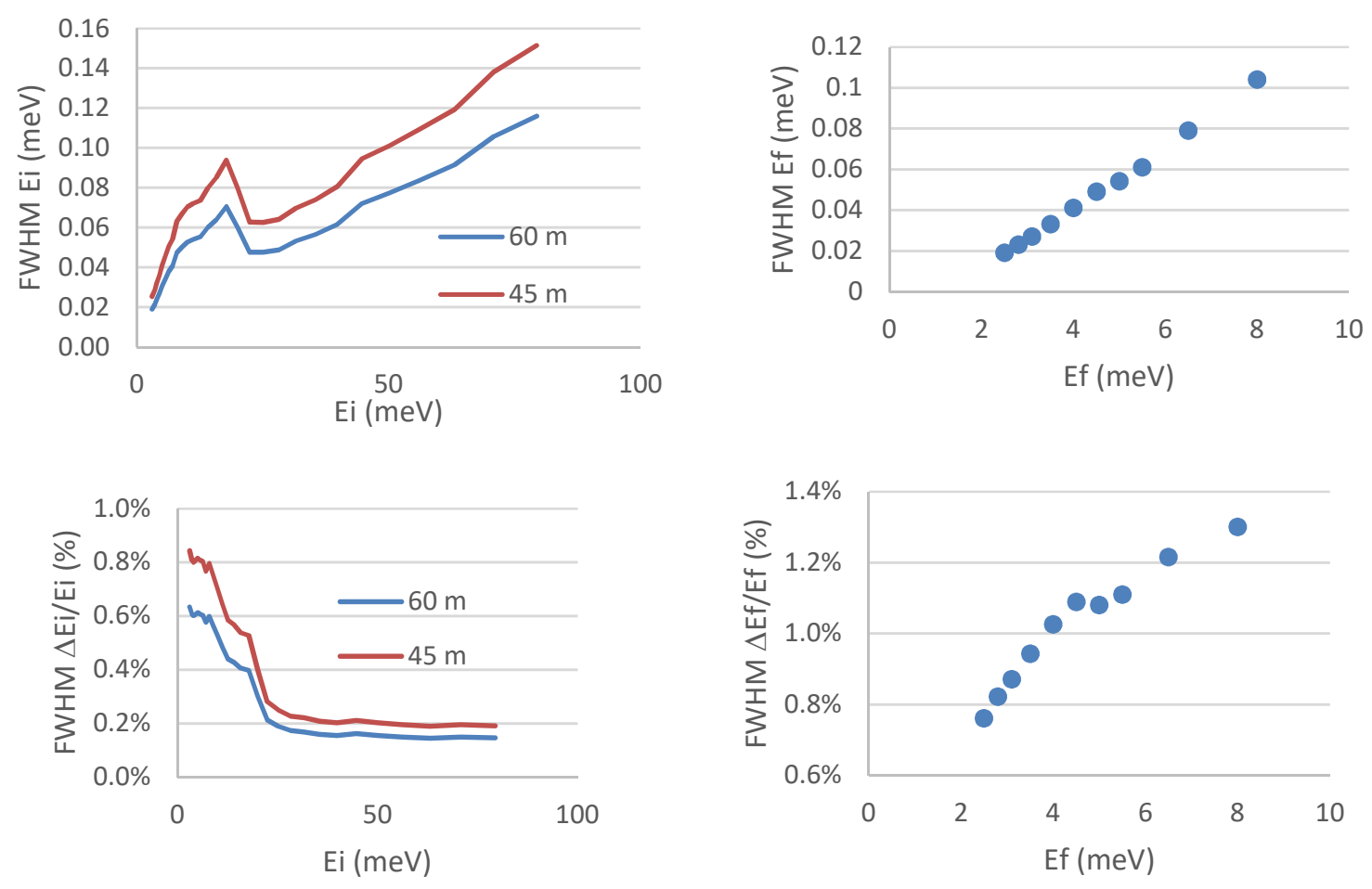

Figure 3.9. Incident and final energy resolution. The incident energy resolution calculation was performed with a source-sample distance of $45 \mathrm{~m}$, and sample diameter $=10 \mathrm{~mm}$. The final energy resolution was obtained from a table describing CAMEA performance parameters and assumes a d-spacing $=3.354 \AA, \Delta \mathrm{d}=0.004 \AA$, sample diameter $=10 \mathrm{~mm}$, analyzer width $=12 \mathrm{~mm}$, analyzer thickness $=1 \mathrm{~mm}$, and a detector diameter $=8 \mathrm{~mm}$.

\subsubsection{Impact of STS Operating Frequency}

As described in section 3.2.2, the scientific drivers behind ZEEMANS naturally select a high brightness source of cold neutrons. The choice of a coupled moderator maximizes flux at sample, but sacrifices timing resolution at $\mathrm{Ei}<15 \mathrm{meV}$ due to broader pulse widths. For the first high field magnet instrument in the US, we choose to optimize the instrument for flux on sample.

The multimodal nature of the beamline makes the optimized STS operating frequency a complicated problem. From bandwidth considerations, assuming we can position sample at $45 \mathrm{~m}$ from the moderator, and given the desired IGS incident energy range of $2.5 \mathrm{meV}<\mathrm{E}_{\mathrm{i}}<80 \mathrm{meV}$, a $15 \mathrm{~Hz}$ mode of operation matches best to the desired range, compared to $20 \mathrm{~Hz}$. For diffraction, should we desire to use nearly the full wavelength bandwidth specified of $0.64 \AA<1<10 \AA$, we would benefit from $7.5 \mathrm{~Hz}$ operation.

\subsubsection{Technical Maturity}

The primary spectrometer and diffraction banks will employ established technologies which are technically mature. The CAMEA spectrometer is being actively developed for the BiFROST instrument at ESS and we will benefit from these developments. However, generation of spurious scattering events and impact on instrumental background pose a moderate risk and will be actively investigated by ORNL. We note that a prototype is being tested at the Paul Sherrer Institut.

The main technical risk for ZEEMANS concerns the magnet itself. Although HTS technology shows incredible promise, only one HTS / low temperature superconducting (LTS) magnet is even approaching 
user program viability (the $32 \mathrm{~T}$ magnet in commissioning at the NHMFL), and the most promising technology (no-insulation ReBCO) is at an earlier stage in development. Several critical technology elements that comprise our desired magnet will require significant scrutiny and maturation.

Given these challenges, an instrument advisory team was formed early for this instrument, and collaboration with NHMFL has already begun. Part of the advisory team, with significant representation from technical subject matter experts for magnet technology and neutron scattering instrumentation, met on August 19, 2016. This team proposed a series of recommendations for magnet development with the goal of maturing the ReBCO technologies. In addition, NHMFL is actively testing no-insulation ReBCO coil systems in ways that will increase technology readiness for the ZEEMANS magnet, and is engaged in a feasibility study for a $35 \mathrm{~T}$ magnet.

Even with appropriate effort to mature these critical technology elements, backup options may still be needed. Backup options employ coil and quench technologies that are more established but which provide a lower maximum magnetic field. Our primary high-risk option is to employ no-insulation ReBCO HTSonly coils. Our moderate-risk option is to employ the insulating ReBCO technology employed in NHMFL's HTS / LTS $32 \mathrm{~T}$ magnet, but plan for a lower maximum field of about $22 \mathrm{~T}$ in split coil geometry. Our low-risk option is to purchase a dedicated LTS-only magnet from a commercial vendor, with a maximum field at or under $16 \mathrm{~T}$. All options described can be accommodated by the external building's space and utility requirements already envisioned for ZEEMANS. Should we choose one of these backup options, then we fully intend to pursue as a separate later project the development of a higher field magnet to replace the one incorporated into the STS project, and then introduce the more portable LTS commercial magnet to the user program for other instruments.

\subsubsection{References}

[1] "Early Scientific Opportunities at the Second Target Station", ORNL Technical Report Draft, 2017.

[2] P.G. Freeman et al., "CAMEA ESS-The continuous angle multi-energy analysis indirect geometry spectrometer for the European Spallation Source", EPJ Web of Conferences 83, 03005 (2015).

[3] M. Bartkowiak et al., "The design of the inelastic neutron scattering mode for the Extreme Environment Diffractometer with the 26 T High Field Magnet", Nuclear Instruments and Methods in Physics Research A 797, 121-129 (2015).

[4] P. Smeibidl et al., "First Hybrid Magnet for Neutron Scattering at Helmholtz-Zentrum Berlin," IEEE Transactions on Applied Superconductivity, vol. 26, no. 4, p. 4301606, 2016.

[5] H. W. Weijers et al., "Progress in the Development and Construction of a 32-T Superconducting Magnet," IEEE Transactions on Applied Superconductivity, vol. 26, no. 4, p. 4300807, 2016.

[6] S. Yoon et al., "26T 35mm all-GdBaCuO multi-width no-insulation superconducting magnet," Superconductivity Science and Technology, vol. 29, p. 04LT04, 2016.

[7] "CAMEA - Continuous Angle Multi-Energy Analysis - Spectrometer Concept Phase Report: ESS Instrument Construction Proposal”, internal technical report, 2014.

[8] E. Mamontov et al., "A time-of-flight backscattering spectrometer at the Spallation Neutron Source, BASIS", Review of Scientific Instruments 82, 085109 (2011). 


\title{
3.3 EWALD: AN EXTENDED WIDE ANGLE LAUE DIFFRACTOMETER FOR THE SECOND TARGET STATION OF THE SPALLATION NEUTRON SOURCE
}

\begin{abstract}
Visualizing $\mathrm{H}$ atoms in biological materials is one of the biggest remaining challenges in biophysical analysis. While X-ray techniques have unrivaled capacity for high-throughput structure determination, neutron diffraction is uniquely sensitive to $\mathrm{H}$-atom positions in crystals of biological materials and can provide a more complete picture of the atomic and electronic structures of biological macromolecules. This information can be essential in providing predictive understanding and engineering control of key biological processes for example, in catalysis, ligand binding, and light harvesting and to guide bioengineering of enzymes and drug design. The relatively low flux on current neutron atomic resolution single crystal diffractometers restrict the technique to studies of crystals with at least $0.1 \mathrm{~mm}^{3}$ sample volumes. Reducing the required size by an order of magnitude or more to less than $0.01 \mathrm{~mm}^{3}$ will open new frontiers for development of improved drugs against multi-resistant viruses and bacteria, understanding enzyme mechanisms and the regulation of metabolic pathways for synthetic biology. Ewald will be a neutron diffractometer that uses the high brightness of STS coupled to a novel optics system to pinpoint hydrogen locations in the structures of macromolecules using single crystals with volumes below $0.01 \mathrm{~mm}^{3}$.
\end{abstract}

\subsubsection{Introduction}

Single crystal neutron diffraction experiments have historically been limited by the available neutron flux, which has always been orders of magnitude lower than available X-ray fluxes. Currently, several instruments such as $\mathrm{LADI}^{1}$, IMAGINE ${ }^{2}, \mathrm{BIODIFF}^{3}$ and IBIX ${ }^{4}$ are now available for single-crystal macromolecular neutron crystallography which can collect data on crystal volumes between 1 to $0.1 \mathrm{~mm}^{3}$. The macromolecular neutron diffractometer ${ }^{5,6}$ (MaNDi) is such an instrument at the First Target Station (FTS) of the Spallation Neutron Source (SNS). FTS produces discrete pulses of neutrons at $60 \mathrm{~Hz}$. Three neutron choppers select the neutron wavelengths which will be used in the experiment from these within these pulses. Scattered neutrons are measured by a large array of detectors which surround the sample that determine the neutron arrival time. On MaNDi which at $60 \mathrm{~Hz}$ has a bandwidth of $\Delta \lambda=2.16 \AA$, neutrons between 2-4.16 $\AA$ are most commonly used in macromolecular experiments. The neutron wavelength is determined by measuring its time-of-flight (TOF) ${ }^{7}$. This enables the Laue diffraction patterns produced on MaNDi to be sorted into monochromatic slices massively reducing reflection overlap, decreasing background and thereby increasing the signal to noise ratio (Figure 3.10). 


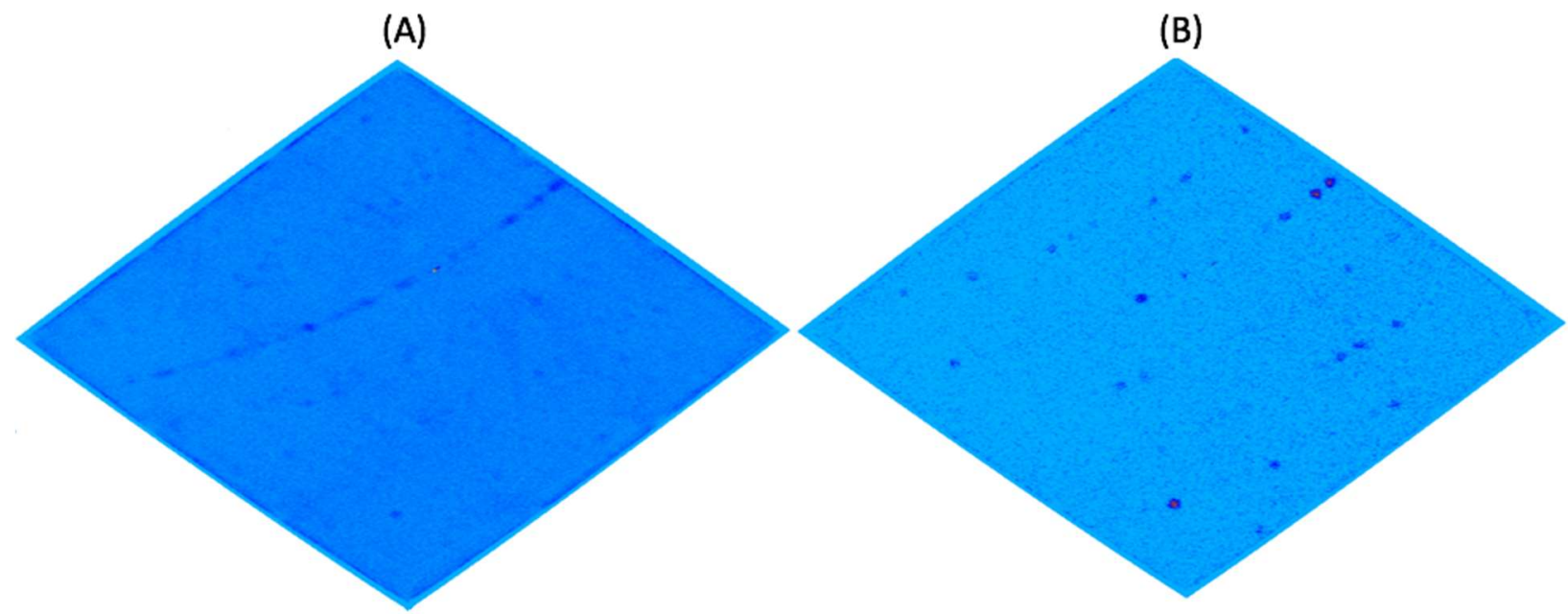

Figure 3.10: Laue diffraction image from a single SNS Anger camera. (A) As recorded on the MaNDi instrument using all wavelengths between 2-4 A. (B) The same detector and diffraction pattern this time looking at neutrons between 3.0-3.1 $\AA$. As can be seen time-of-flight or wavelength resolution significantly reduces reflection overlap enabling data collection from large unit cell axes while also significantly lowering background.

Due to its high TOF resolution ${ }^{8}$ MaNDi can collect data from unit cells up to $300 \AA$ shile also collecting data on crystal volumes down to $0.1 \mathrm{~mm}^{3}$. However, improved neutron sources and instrumentation are needed to develop the field and move to crystal volumes an order of magnitude smaller for data collection. The high brightness of cold neutrons that will be provided by the SNS Second Target Station (STS) is ideal for building an instrument that uses novel focusing optics to illuminate a small sample with high flux. This capability will enable the study of proteins from which it is challenging to prepare crystals as large as $0.1 \mathrm{~mm}^{3}$ in volume. Ewald is a high resolution macromolecular single crystal diffractometer designed for collecting data from large unit cells up to $300 \AA$ on edge and is optimized for crystal volumes of less than $0.01 \mathrm{~mm}^{3}$. When coupled to the high brightness STS moderator, Montel (nested) Kirkpatrick-Baez (KB) neutron supermirrors ${ }^{9,10}$ will deliver the small, intense neutron beams EWALD requires.

\subsubsection{Instrument Concept}

The neutron optics system for Ewald (Figure 3.11) is based on a pair of nested elliptical KB focusing neutron supermirrors located at $54 \mathrm{~m}$ and $84 \mathrm{~m}$ from the moderator. These neutron supermirrors are $3 \mathrm{~m}$ in length and $15 \mathrm{~cm}$ in height and image a neutron slit which will be positioned $5 \mathrm{~m}$ from the moderator. The opening of this slit will be de-magnified (focused) by a factor of x30 (in area) at the sample position, by varying the size of the slit opening we will be able to adjust the dimensions of the neutron beam at the sample position down to $0.001 \mathrm{~mm}^{2}$. Using this mechanism, we will be able to closely match the beam size at the sample position to match the dimensions of the crystal to reduce background and increase signal to noise. Using this arrangement, we also have no line of sight from the moderator to the sample position further reducing background while also avoiding any fast neutrons and gammas produced during neutron production reaching the sample position even in the event of a chopper failure. The horizontal and vertical divergence of the neutron beam at the sample is fixed at $0.38^{\circ} \mathrm{FWHM}$ across the $1-10 \AA$ wavelength band. 


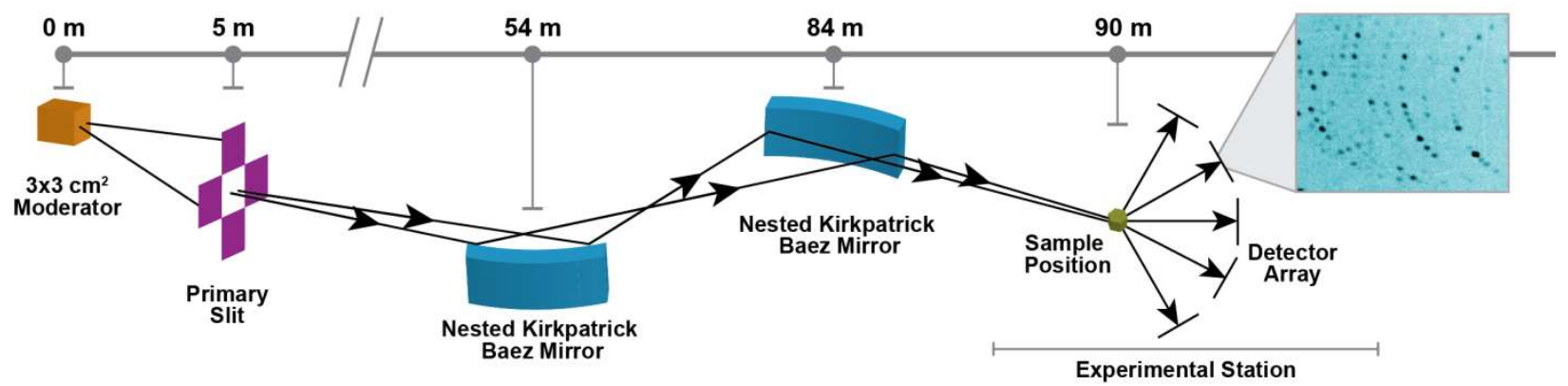

Figure 3.11. A schematic overview of the neutron optics on Ewald showing the location of the moderator, primary slit, Kirkpatrick-Baez (KB) neutron supermirrors, sample position, detector array and the diffraction pattern from a single detector module.

A T0 chopper located at $6.5 \mathrm{~m}$ and two bandwidth choppers positioned at $8 \mathrm{~m}$ and $10 \mathrm{~m}$ from the moderator will remove fast neutrons and gammas and select the neutron wavelengths to be used for each experiment. A secondary shutter located at $84.6 \mathrm{~m}$ will allow the easy change out of samples. At the sample position $(90 \mathrm{~m})$ a high precision goniometer will align and position the crystal into the neutron beam with neutrons scattered from the sample being detected by a semi spherical array of next generation high resolution SNS Anger camera detectors based on silicon photo-multiplier technology.

MaNDi $^{5,11}$ views a poisoned, decoupled hydrogen moderator at the SNS first target station which gives sharp neutron pulses with short emission times ${ }^{8}(17.4 \mu$ sec FWHM at $2 \AA)$ enabling the study of large unit cell axes up to $300 \AA^{8}$. To ensure the same or better wavelength resolution for Ewald at the STS which views a $3 \times 3 \mathrm{~cm}^{2}$ high brightness coupled moderator one needs an instrument that is around three times longer to account for the moderator pulse width difference ( $43.3 \mu \mathrm{sec}$ FWHM at $2 \AA)$. Thus, Ewald has a flight path length (moderator-sample) of $90 \mathrm{~m}$, three times that of MaNDi. At this length and with the $15 \mathrm{~Hz}$ repetition rate of the STS Ewald will have a bandwidth $(\Delta \lambda)$ of $3 \AA$, perfect for neutron protein crystallography as all useful wavelengths (1.5-4.5 $\AA$ ) can be used in a single frame.

\subsubsection{Neutron Flux}

Using the McStas ${ }^{12}$ program we have conducted several initial Monte Carlo simulations of Ewald to assess its performance relative to that of MaNDi at the first target station of SNS. On MaNDi ${ }^{5}$ with a fixed beam divergence of $0.38^{\circ}$ at the sample position the flux on the sample is $1.3 \times 10^{5} \mathrm{n} \mathrm{s}^{-1} \mathrm{~mm}^{-2}$ for all neutrons between 2-4.16 $\AA$. The higher brightness coupled moderator available at the STS coupled with a $\mathrm{KB}$ neutron optics system has enabled us to increase the flux on sample at Ewald to $7.64 \times 10^{6} \mathrm{n} \mathrm{s}^{-1} \mathrm{~mm}^{-2}$ for all neutrons between 1.5-4.5 $\AA$ with a fixed beam divergence of $0.38^{\circ}$ at the sample position which gives us a simulated gain factor in flux of $x 59$.

We have simulated the performance of Ewald on a small crystal $\left(0.01 \mathrm{~mm}^{3}\right)$ of an Inorganic pyrophosphatase (IPPase) using the $\mathrm{McStas}^{12}$ program. IPPase is an enzyme that catalyzes the conversion of one molecule of pyrophosphate to two phosphate ions which is a highly exergonic reaction. This reaction is often coupled to unfavorable biochemical transformations to help drive these transformations to completion. The functionality of this enzyme plays a critical role in lipid synthesis and degradation, bone formation and DNA synthesis. The protein itself is a hexamer in the asymmetric unit formed from six protein chains being composed of 174 amino acids each ${ }^{13}$. This large complex is a challenging target for neutrons and initial data collection at the protein crystallography station (PCS) instrument located on the LANSCE center in Los Alamos, NM required a crystal over 500 times larger in volume $\left(5 \mathrm{~mm}^{3}\right)$ for data collection ${ }^{13}$. 
The specifics of the simulations conducted for Ewald and MaNDi are given below in Table 3.4. A narrow time-of-flight (TOF) range corresponding to neutrons between 2.82 and $2.84 \AA$ was used for the Monte Carlo simulations due to the large number of reflections generated from such as large unit cell.

Table 3.4. Parameters for instrument simulations.

\begin{tabular}{lll}
\hline \multicolumn{1}{c}{ Instrument } & \multicolumn{1}{c}{ Ewald } & \multicolumn{1}{c}{ MaNDi } \\
\hline Wavelength Center $(\AA)$ & 2.83 & 2.83 \\
Simulated Detector Type & HR-SNS Anger Camera & SNS Anger Camera \\
Crystal-to-detector distance $(\mathrm{mm})$ & 300 & 400 \\
Detector Pixel size $\left(\mathrm{mm}^{2}\right)$ & 0.5 & 1 \\
Detector Coverage (SR) & 5.3 & 4.0 \\
Space group & $\mathrm{C} 2$ & $\mathrm{C} 2$ \\
a, b, c $(\AA)$ & $106.10,95.51,113.73$ & $106.10,95.51,113.73$ \\
$\alpha, \beta, \gamma\left({ }^{\circ}\right)$ & $90.00,98.08,90.00$ & $90.00,98.08,90.00$ \\
Resolution range $(\AA)$ & $19.68-1.50$ & $19.68-1.50$ \\
Total No. of possible reflections & 1795,238 & 1795,238 \\
Number of atoms $($ Asymmetric unit) & 18,613 & 18,613 \\
Crystal Volume $\left(\mathrm{mm}^{3}\right)$ & 0.01 & 0.01 \\
Crystal Mosaic Spread $\left({ }^{\circ}\right)$ & 0.3 & 0.3 \\
\hline
\end{tabular}

(B)

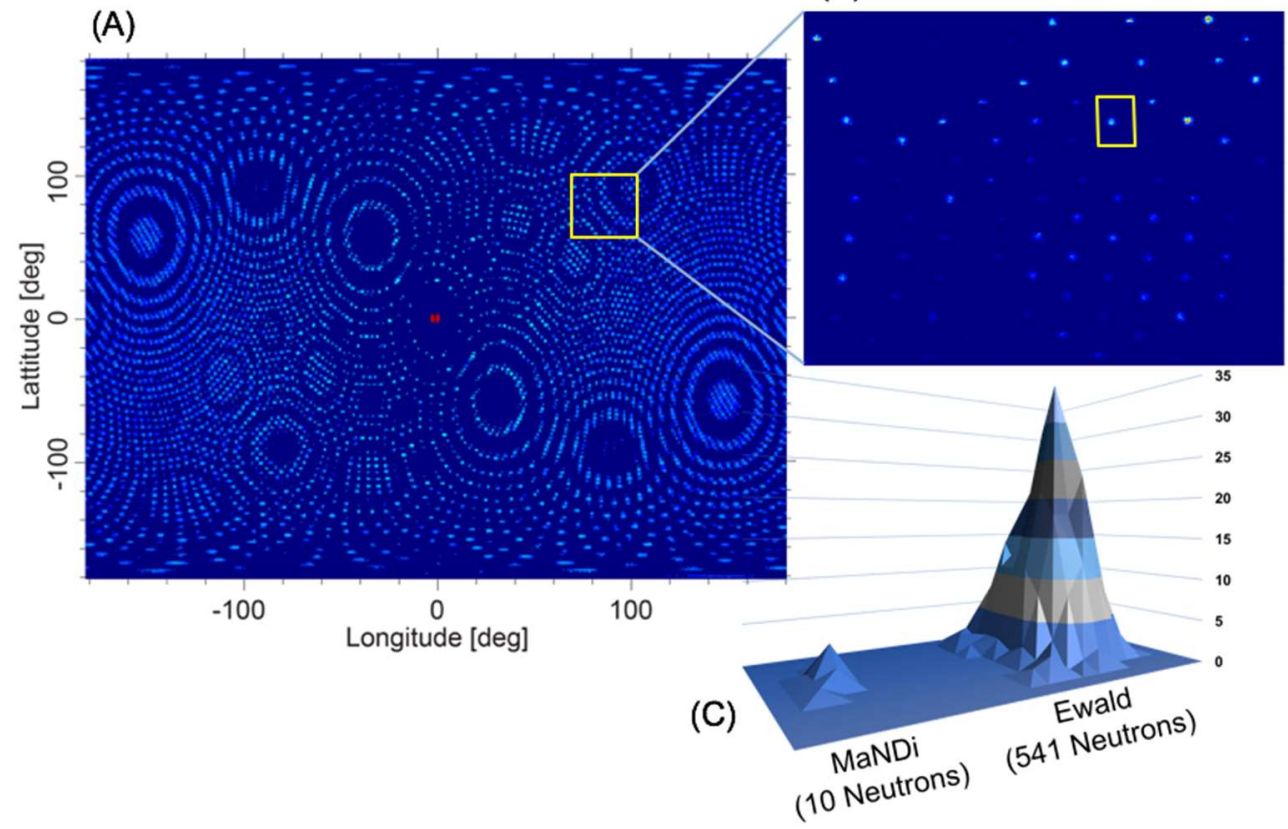

Figure 3.12. Monte Carlo simulated diffraction patterns from a protein crystal $0.01 \mathbf{~ m m}^{3}$ in volume on the

EWALD instrument. (A) The complete set of Bragg diffraction peaks produced by the protein crystal at a hypothetical spherical detector located $300 \mathrm{~mm}$ from the sample. (B) Subset of diffraction peaks as observed by a single high-resolution SNS Anger camera at a $2 \theta$ angle of $90^{\circ}$ is shown for EWALD. (C) Close-up of a simulated Bragg peak corresponding to a resolution of $2.0 \AA$ are shown for both EWALD and the current instrument, MaNDi. The simulated Bragg peak produced from EWALD is composed of 541 neutron counts, compared to just 10 from MaNDi, showing the ability of EWALD to collect meaningful data on protein crystal volumes $0.01 \mathrm{~mm}^{3}$ and below. 


\subsubsection{Dynamical Neutron Polarization}

Dynamical Neutron Polarization (DNP) uses a combination of high magnetic fields and low temperature to enhance and manipulate the nuclear polarization in macromolecular crystals giving the ability to control the neutron cross section ${ }^{14,15}$. In situ control of the neutron cross section will significantly enhance the contribution of Hydrogen which accounts for over half the atoms in a typical protein crystal to the measured signal while simultaneously minimizing the incoherent scattering background. This will potentially further reduce the crystal size required for Ewald by a further order of magnitude (x10). Ewald has been flexibly designed to accommodate DNP equipment and sample environment. The end station of the Ewald instrument is initially designed with 37 HR-SNS anger Cameras (Figure 3.13) which are mounted on a semi spherical movable detector array frame which can be easily retracted to allow for the installation of the DNP apparatus.
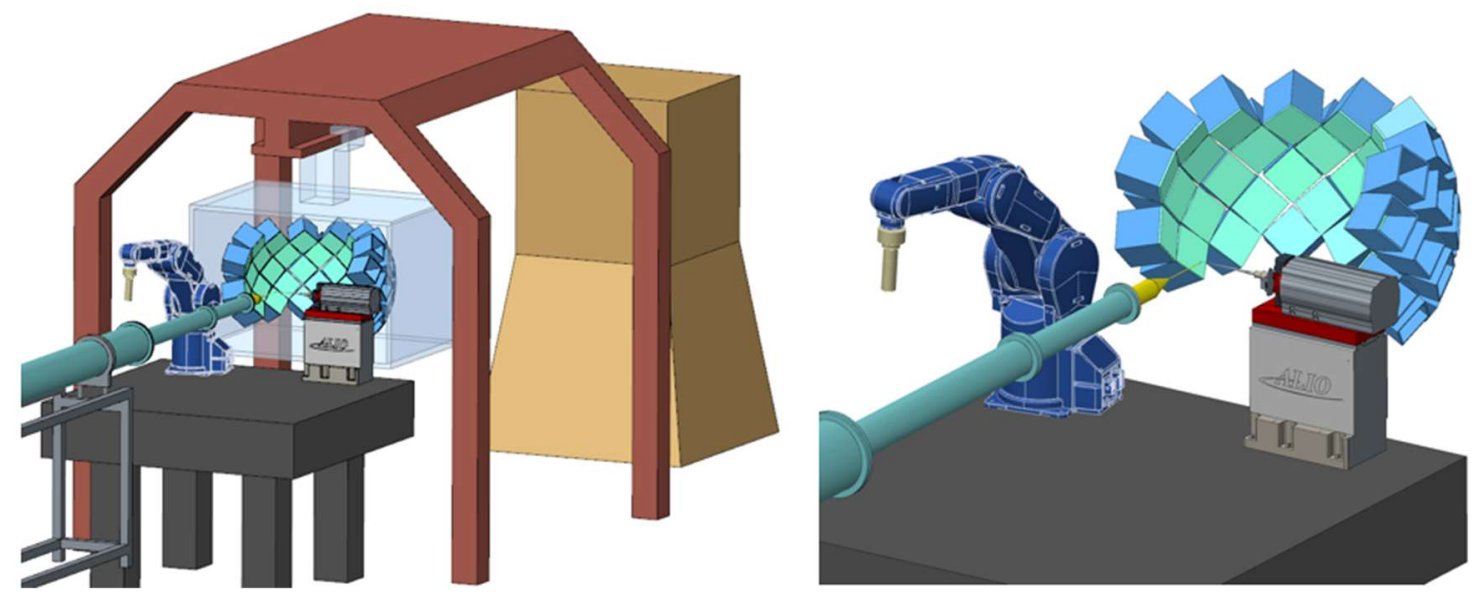

Figure 3.13. The current model of the Ewald end station is shown. A large detector array composed of $37 \mathrm{HR}-$ SNS Anger cameras is installed to reduce the number of orientations required to collect a complete dataset. A highresolution goniometer, nitrogen cryostream and robotic sample changer will allow for offsite operation.

At a sample to detector distance of $300 \mathrm{~mm}$ the detector array provides a coverage of $5.3 \mathrm{sr}$ compared to $4 \mathrm{sr}$ on the MaNDi instrument. Ewald will utilize the next generation of SNS Anger Camera Design which will have a minimum pixel size of $0.5 \mathrm{~mm}^{2}$ which will enable profile fitting of Bragg reflections even from crystals below $0.01 \mathrm{~mm}^{2}$. Unlike the current detectors installed on MaNDi this next generation detector is not sensitive to stray the magnetic fields created by DNP equipment enabling DNP deployment on Ewald. A high precision goniometer at the sample position $(90 \mathrm{~m})$ aligns crystals into the neutron beam while a robotic sample changer allows for automated data collection and remote operation of the beamline. Further work of the instrument design will include modelling of the effects of gravity on the neutron optics system and improving detector coverage.

\subsubsection{Acknowledgements}

This research at ORNL's Spallation Neutron Source was sponsored by the Scientific User Facilities Division, Office of Basic Energy Sciences, U. S. Department of Energy. The Office of Biological and Environmental Research supported research at Oak Ridge National Laboratory's Center for Structural Molecular Biology (CSMB), using facilities supported by the Scientific User Facilities Division, Office of Basic Energy Sciences, U. S. Department of Energy. 


\subsubsection{References}

1. Blakeley, M.P. et al. Neutron macromolecular crystallography with LADI-III. Acta Crystallographica Section D-Biological Crystallography 66, 1198-1205 (2010).

2. Meilleur, F. et al. The IMAGINE instrument: first neutron protein structure and new capabilities for neutron macromolecular crystallography. Acta Crystallographica Section D-Biological Crystallography 69, 2157-2160 (2013).

3. Coates, L. et al. Cryogenic neutron protein crystallography: routine methods and potential benefits. Journal of Applied Crystallography 47, 1431-1434 (2014).

4. Tanaka, I. et al. Neutron structure analysis using the IBARAKI biological crystal diffractometer (iBIX) at J-PARC. Acta Crystallographica Section D-Biological Crystallography 66, 1194-1197 (2010).

5. Coates, L., Stoica, A.D., Hoffmann, C., Richards, J. \& Cooper, R. The macromolecular neutron diffractometer (MaNDi) at the Spallation Neutron Source, Oak Ridge: enhanced optics design, highresolution neutron detectors and simulated diffraction. Journal of Applied Crystallography 43, 570577 (2010).

6. Coates, L. et al. The Macromolecular Neutron Diffractometer MaNDi at the Spallation Neutron Source. Journal of Applied Crystallography 48, 1302-1306 (2015).

7. Langan, P. et al. Protein structures by spallation neutron crystallography. Journal of Synchrotron Radiation 15, 215-218 (2008).

8. Schultz, A.J. et al. Conceptual design of a macromolecular neutron diffractometer (MaNDi) for the SNS. Journal of Applied Crystallography 38, 964-974 (2005).

9. Ice, G.E., Barabash, R.I. \& Khounsary, A. Nested mirrors for X-rays and Neutrons. Advances in XRay/Euv Optics and Components Iv 7448(2009).

10. Ice, G.E. et al. Design challenges and performance of nested neutron mirrors for microfocusing on SNAP. Journal of Applied Crystallography 42, 1004-1008 (2009).

11. Coates, L. et al. The Macromolecular Neutron Diffractometer MaNDi at the Spallation Neutron Source. Journal of Applied Crystallography 48(2015).

12. Willendrup, P.K. et al. New developments in the McStas neutron instrument simulation package. International Workshop on Neutron Optics and Detectors (Nop\&D 2013) 528(2014).

13. Hughes, R.C. et al. Inorganic pyrophosphatase crystals from Thermococcus thioreducens for X-ray and neutron diffraction. Acta Crystallographica Section F-Structural Biology and Crystallization Communications 68, 1482-1487 (2012).

14. Pierce, J. et al. Dynamically polarized sample at the Spallation Neutron Source. International Conference on Neutron Scattering 2009 251(2010).

15. Pierce, J., Crabb, D. \& Zhao, J.K. Dynamically Polarized Sample for Neutron Scattering At the Spallation Neutron Source. Spin Physics 1149, 872-875 (2009). 


\subsection{THE QUITE INTENSE KINETICS REFLECTOMETER (QIKR)}

\subsubsection{Overview}

The Quite Intense Kinetics Reflectometer (QIKR) at the Spallation Neutron Source (SNS) Second Target Station (STS) is envisioned as a short-flight-path instrument to utilize the low STS repetition frequency and optimized thermal and cold neutron instantaneous flux in a broad wavelength band to carry out complete specular reflectivity measurements at a single incident angle. Operated this way, QIKR functions as a specular reflectivity motion-picture camera. To perform an experiment: align the sample, set the beam incident angle and apertures, and start counting. The sample can then be subjected to timevarying stimulus without necessarily requiring it to be in a stable state - the camera is running the entire time, collecting the full $\mathrm{Q}$ range. The event-mode data can be processed post-experiment to look at environment stable states and the transitions between them, e.g. observing a potentiostat charging/discharging a battery cathode/anode or observing a diffusion-limited chemical modification of a polymer thin film. Alternatively, the event-mode data measured from a sample exposed to a continuously varying asynchronous stimulus (e.g. a rheometer) can be subdivided into constant parameter-value (e.g., angular velocity) data sets.

\subsubsection{Concept}

\subsubsection{Dynamic Range}

The kinetic performance of a specular reflectometer is straightforward to describe. By manipulating $Q=$ $4 \pi \sin \theta / \lambda$ and the kinematic equations describing neutron time-of-flight, $h^{2} / 2 m \lambda^{2}=m v^{2} / 2$, the dynamic range of a measurement at a single instrument setting can be described in terms of Planck's constant $h$, neutron mass $m$, source-detector distance $L$, source repetition frequency $f$, and the minimum neutron wavelength, $\lambda_{\min }$, in the selected band,

$$
\frac{Q_{\max }}{Q_{\min }}=1+\frac{h}{m} \frac{1}{L} \frac{1}{f} \frac{1}{\lambda_{\text {min }}} \equiv D .
$$

Dynamic range $D$ (decibels) is maximized for short-flight-path instruments on low frequency neutron sources featuring peak flux at short wavelengths (note $h / m=3956.0 \mathrm{~m}-\mathrm{Hz}-\AA$ ) . 


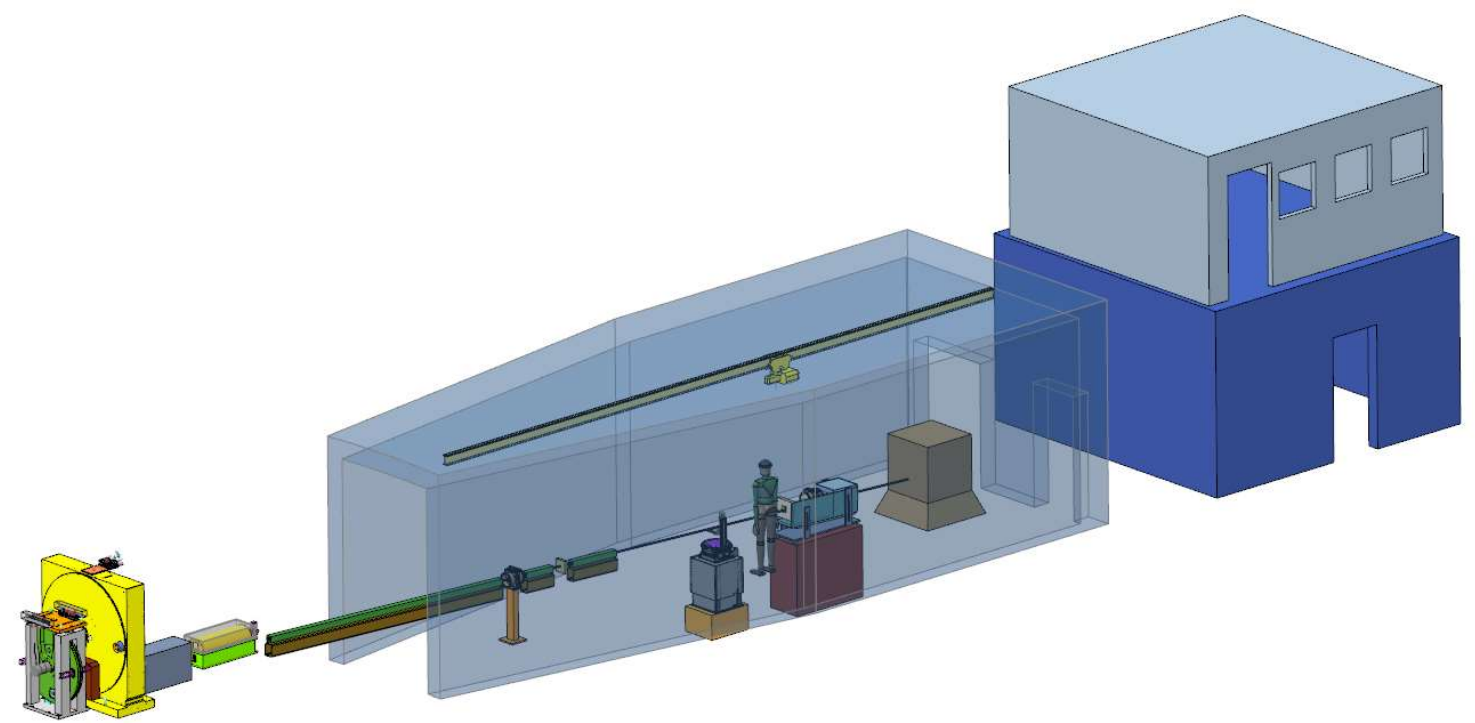

Figure 3.14. Engineering model of QIKR.

Conceptual design is ongoing for QIKR, but an instrument featuring $L=20 \mathrm{~m}, f=7.5 \mathrm{~Hz}$ (pulse- skipping on a $15-\mathrm{Hz}$ source), and $\lambda_{\min }=2.5 \AA$, consistent with current neutron source and target building constraints, yields $D=11.5$. Neutron disk choppers can extract $90-95 \%$ of the theoretical dynamic range, so $D_{\text {eff }}=10.3$. At an incident angle $\theta=2.5^{\circ}$, one will thus be able to measure $0.021 \AA^{-1}<Q<0.219 \AA^{-1}$. Different incident angles yield different $Q$ ranges, but $D$ remains the same.

\subsubsection{Reflection Geometries}

While the $Q$ range shown above may suffice for most experiments, there will be instances where smaller and/or greater wave vectors are needed. QIKR will be able to perform these measurements as well. There are two basic geometries for reflectivity measurements: $\theta-2 \theta$ in which the sample is tilted relative to a fixed incident beam (e.g. for solid samples and samples in liquid/solid cells) and the $\theta-\theta$ geometry for samples which cannot be tilted, such as free liquid surfaces and liquid/liquid interfaces. Implementing the $\theta-2 \theta$ geometry is straightforward, simply requiring accurate $\theta$ and $2 \theta$ positioning around a fixed incident beam. Measuring in the $\theta-\theta$ geometry, however, requires one to vary the angle of the incident beam against a horizontal surface. Fortunately, the large QIKR wavelength bandwidth $(2.5 \AA<\lambda<23.8 \AA)$ and the divergence afforded by a tapered neutron guide enable one to cover $0.009 \AA^{-1}<Q<0.351 \AA^{-1}$ in two instrument settings (see below the measured output of the $m=3.6$ SNS Liquids Reflectometer tapered guide plotted about a $2.5^{\circ}$ centerline and a table showing $Q$ ranges for different $\theta$ values). 


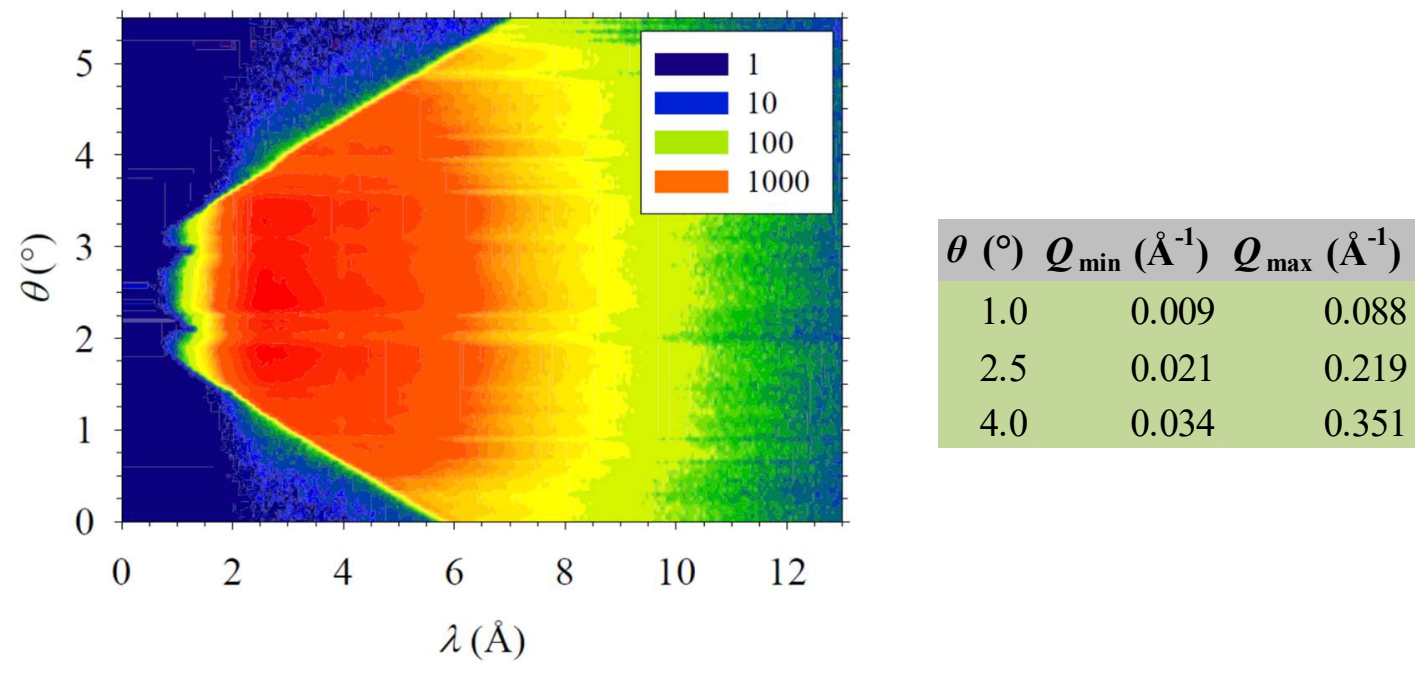

Figure 3.15. Measured output of the SNS Liquids Reflectometer tapered guide.

\subsubsection{Two Separate Beams}

For liquid/liquid measurements in which the denser phase is also the hydrogen-poor phase or for sample environments such as cone-and-plate rheometers, the neutron beam must strike the interface from below. It is not possible, using current guide technology, to divert a single neutron beam from $-2.5^{\circ}$ to $+2.5^{\circ}$ without unacceptable losses, particularly at shorter wavelengths. Such losses rapidly decrease dynamic range $D$ by increasing $\lambda_{\min }$. We will therefore employ two distinct incident beams with independent neutron guides. The beams diverge from the moderator and are well separated at the entrance to the chopper bunker (separation $s=0.49 \mathrm{~m}$ at distance $d=5.60 \mathrm{~m})$. Within the chopper bunker $(5.6 \mathrm{~m}<d<$ $11.2 \mathrm{~m}$ ), the $T_{0}$ chopper and single bandwidth chopper will be mounted exactly between the two diverging guides and will chop one or the other beam depending on their phasing. The beam shutter, also located within the bunker, will select which beam is viewed. The guides emerge into the instrument cave from bulk shielding at $d=16.0 \mathrm{~m}$. An incident optics table, carrying e.g. slits and frame-overlap mirror, will be positioned in height and angle to intercept the desired beam. The sample and detector will have to travel a significant height to switch between beams and both may need to be recessed below floor level to accommodate this travel. The figure below shows a "revolver"-type shutter concept and a table of beam height separation $s$ and the heights, $h_{ \pm 2.5}$, of the two beams from the floor vs. distance from the moderator $d$. 


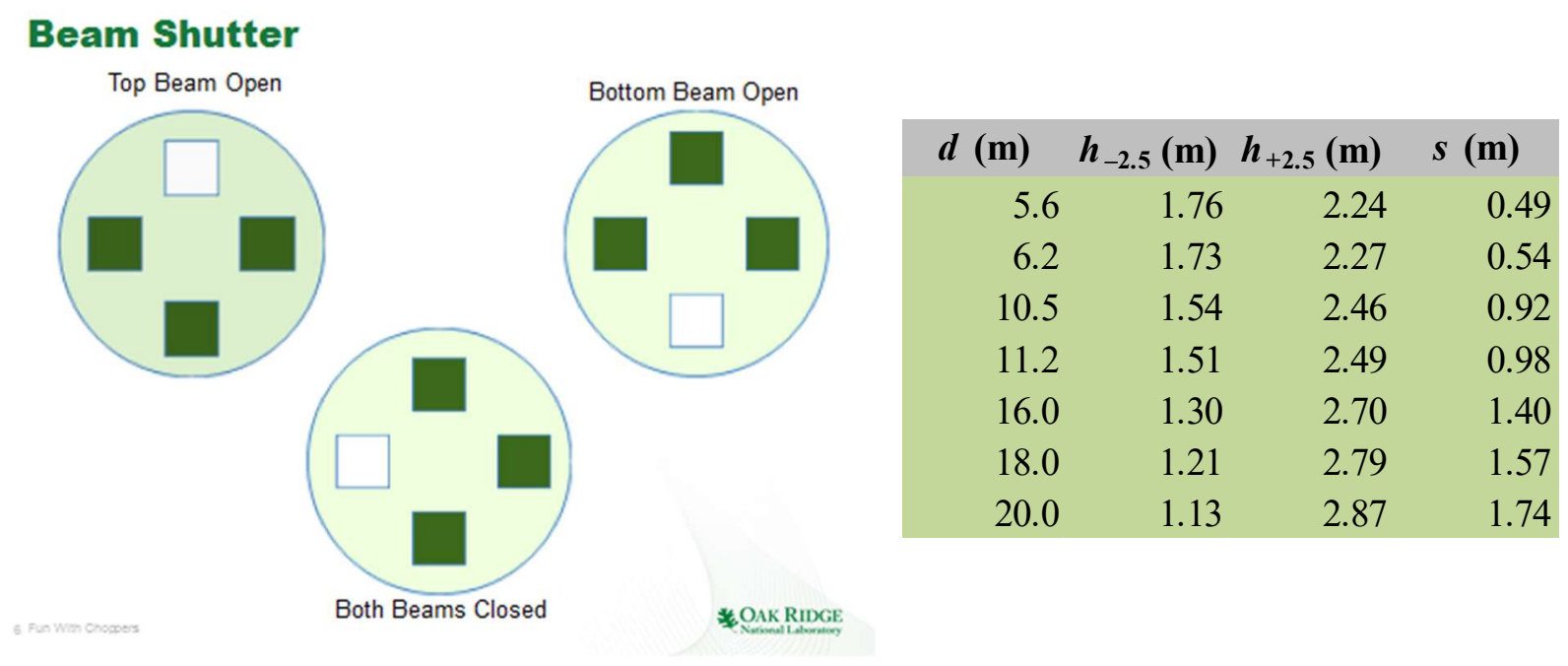

Figure 3.16. QIKR shutter concept and table of beam heights vs distance from the moderator.

\subsubsection{Challenge}

What phenomena could you capture with such a camera?

\subsubsection{Example}

Polymer diffusion is a subject of ongoing interest, e.g., in compatibilization, phase separation in organic photovoltaics, or in the structure and properties of polyelectrolyte-based hydrogels. Selective deuteration affords neutron reflectivity a unique view into these processes. A typical example of a film used in these studies consists of alternating layers of deuterated and conventional polymer (polystyrene in this case) deposited on a silicon substrate.

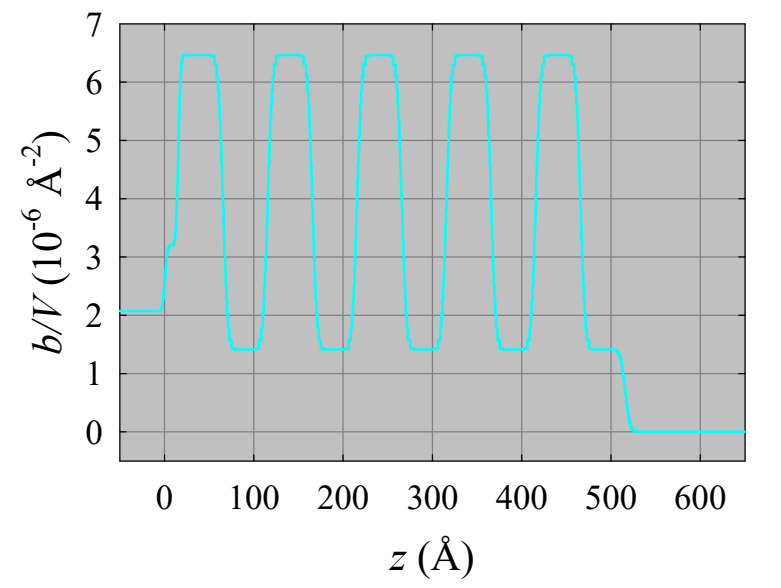

\begin{tabular}{|c|c|c|c|c|c|c|}
\hline Compound & $N b\left(\AA^{-2}\right)$ & $\begin{array}{l}\text { Model Para } \\
\mu_{l}{ }^{\text {abs }} / \lambda\left[\AA^{-2}\right]\end{array}$ & $\mu_{I}^{\text {inc }}\left[\AA^{-1}\right]$ & $d(\AA)$ & $\sigma(\AA)$ & \\
\hline air & 0.00 & 0.00 & 0.00 & 200.0 & 0.0 & $\sigma$ PS-dPS $(\AA)$ \\
\hline dPS & $1.41 \mathrm{E}-06$ & $9.08 \mathrm{E}-11$ & $3.90 \mathrm{E}-08$ & 50.0 & 10.0 & 10.0 \\
\hline PS & $6.47 \mathrm{E}-06$ & $1.09 \mathrm{E}-12$ & $9.96 \mathrm{E}-10$ & 50.0 & 10.0 & $d_{\mathrm{PS}}(\AA)$ \\
\hline dPS & $1.41 \mathrm{E}-06$ & $9.08 \mathrm{E}-11$ & $3.90 \mathrm{E}-08$ & 50.0 & 10.0 & 50.0 \\
\hline PS & $6.47 \mathrm{E}-06$ & $1.09 \mathrm{E}-12$ & $9.96 \mathrm{E}-10$ & 50.0 & 10.0 & \\
\hline dPS & $1.41 \mathrm{E}-06$ & $9.08 \mathrm{E}-11$ & $3.90 \mathrm{E}-08$ & 50.0 & 10.0 & \\
\hline PS & $6.47 \mathrm{E}-06$ & $1.09 \mathrm{E}-12$ & $9.96 \mathrm{E}-10$ & 50.0 & 10.0 & \\
\hline dPS & $1.41 \mathrm{E}-06$ & $9.08 \mathrm{E}-11$ & $3.90 \mathrm{E}-08$ & 50.0 & 10.0 & \\
\hline PS & 6.47E-06 & 1.09E-12 & $9.96 \mathrm{E}-10$ & 50.0 & 10.0 & \\
\hline dPS & $1.41 \mathrm{E}-06$ & $9.08 \mathrm{E}-11$ & $3.90 \mathrm{E}-08$ & 50.0 & 10.0 & \\
\hline PS & $6.47 \mathrm{E}-06$ & $1.09 \mathrm{E}-12$ & $9.96 \mathrm{E}-10$ & 50.0 & 10.0 & \\
\hline $\mathrm{SiOx}$ & $3.20 \mathrm{E}-06$ & $9.77 \mathrm{E}-13$ & $8.96 \mathrm{E}-10$ & 15.0 & 5.0 & \\
\hline $\mathrm{Si}$ & 2.07E-06 & $4.75 \mathrm{E}-11$ & $2.00 \mathrm{E}-12$ & 100.0 & 5.0 & \\
\hline
\end{tabular}

Figure 3.17. Parameters for alternating layers of deuterated and conventional polystyrene. 
The width of the interfacial mixing zone between deuterated and conventional layers, tracked as a function of time, can be used to determine the diffusion coefficient of the polymer. Currently, these measurements are performed in a quenched state, e.g. at room temperature after annealing in a furnace or under solvent vapor, since measurement out to $Q=0.2 \AA^{-1}$ requires an hour or more counting time. For the current version of the QIKR instrument, viewing the $5 \times 5 \mathrm{~cm}^{2}$ coupled $\mathrm{H}_{2}$ STS moderator, with instrument resolution $\delta Q / Q=0.025, \theta=2.5^{\circ}$, and $2.5 \AA<\lambda<23.6 \AA$, the following data may be collected in a single setting at the indicated different counting times.

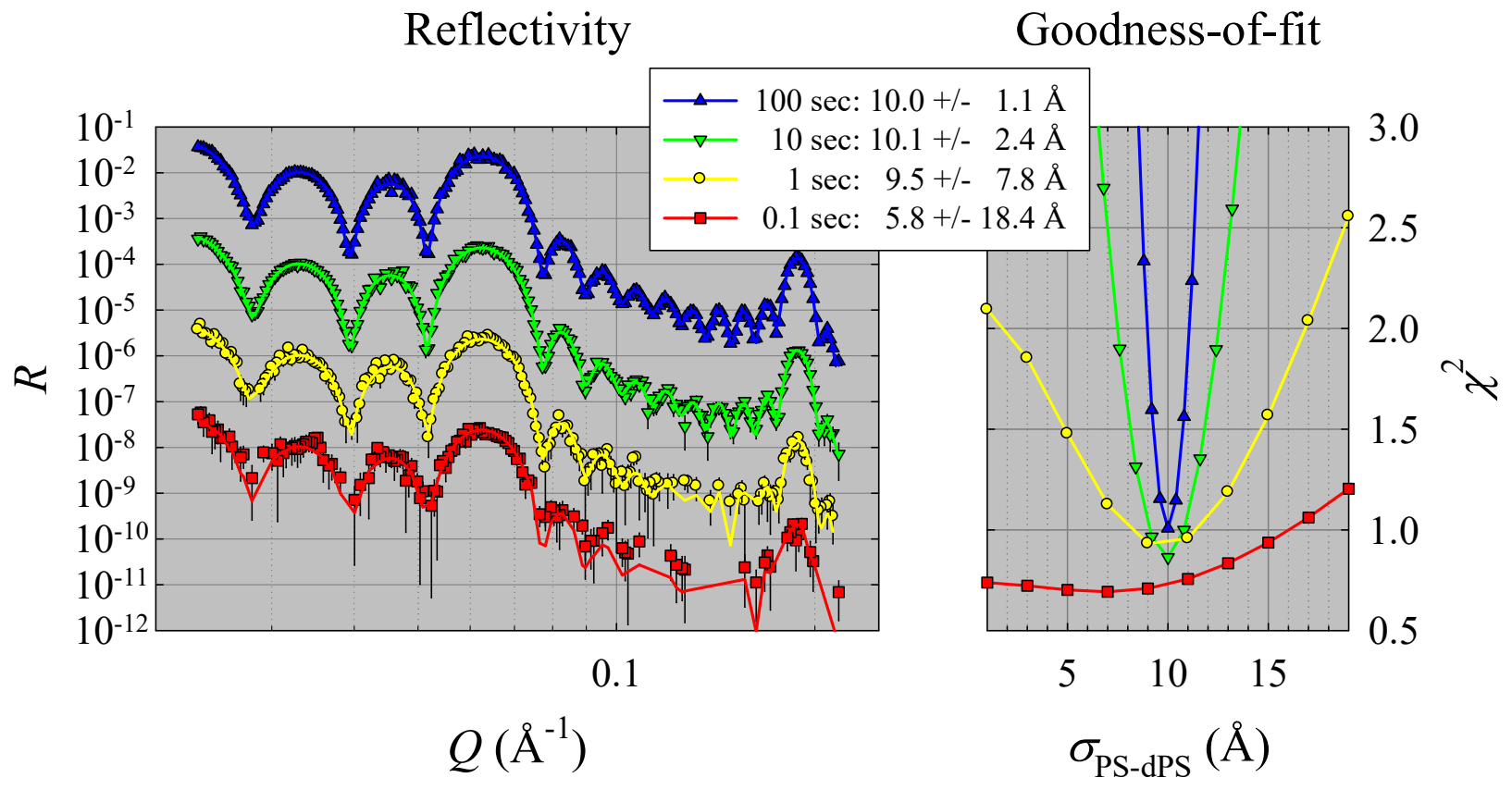

Figure 3.18. Simulated QIKR data for polymer diffusion study.

Depending on the required statistical accuracy, a valid estimate of the interfacial width can be made in a little as 10 seconds; the bilayer thickness can be determined to $\pm 1 \AA$ with a single proton pulse $(0.1 \mathrm{sec})$.

\subsubsection{First-Day Experiment: Kinetics of EDA crosslinking in multilayer films of PMAA (Prof. E. Kharlampieva, U. Alabama, Birmingham)}

We have shown in recent experiments on responsive hydrogels that multilayer films of polymethacrylic acid (PMAA) assembled through hydrogen bonded layer-by-layer adsorption with polyvinylpyrrolidone (PVPON) on surfaces can be crosslinked with ethylenediamine (EDA) and the PVPON subsequently released at high $\mathrm{pH}$ to leave a crosslinked PMAA hydrogel [ACS Macro Lett. 2, 226 (2013)]. However, the events that occur on small timescales during the crosslinking have yet to be fully characterized and as such remain a fundamental inquiry in this type of assembly. Specifically, addition of EDA to the network was shown to ionize the PMAA's acid groups that were not activated by the carbodiimide. FTIR analysis showed that this ionization had already occurred significantly in the timescale $(\sim 2 \mathrm{~min})$ we operated under to take the measurement (Figure 3.19). Ionization of PMAA necessarily disrupts the film stability as ionized COO-groups cannot participate in the same H-bonding that the protonated groups can. However, using neutron reflectometry we have shown that spin-coated multilayer films were highly organized after crosslinking and removal of the non-crosslinked component [Macromolecules $\underline{49}, 6953$ 
(2016)] (Figure 3.20). This structural persistence implies that the transition between activated, noncrosslinked PMAA and crosslinked PMAA must happen on a timescale shorter than or very close to the induced ionization by the EDA. Therefore, we would like to study the time-resolved conversion at subminute resolution of the hydrogen bonded film with activated $\mathrm{COOH}$ groups to crosslinked PMAA with EDA-induced ionization by probing the film's structure with neutrons during this time interval.

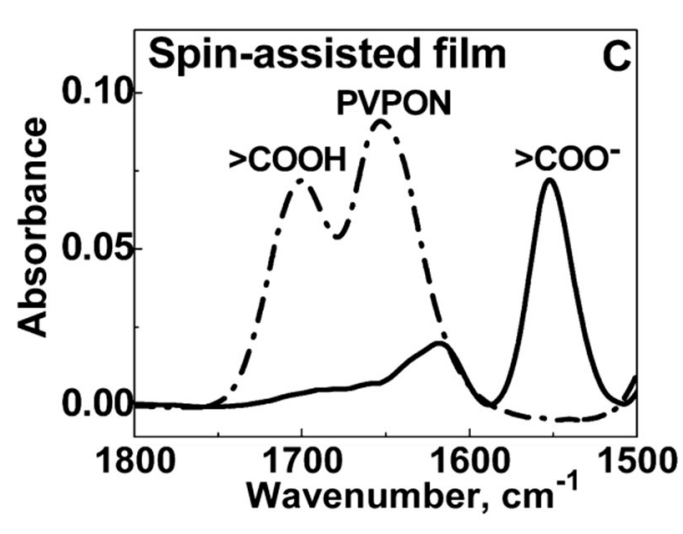

Figure 3.19. Ionization of PMAA in the crosslinking of the multilayer film
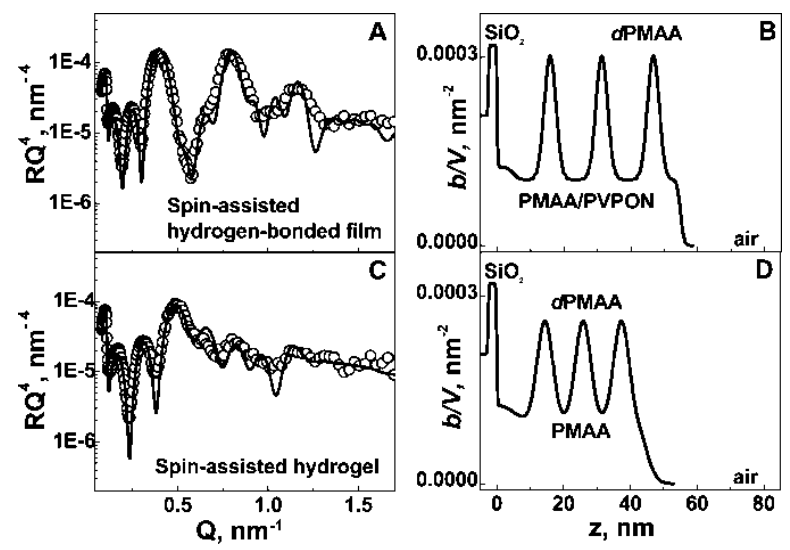

Figure 3.20. Reflectivity and SLD plots of spinassisted PMAA multilayers H-bonded with PVPON (top) and after crosslinking/release of PVPON(bottom).

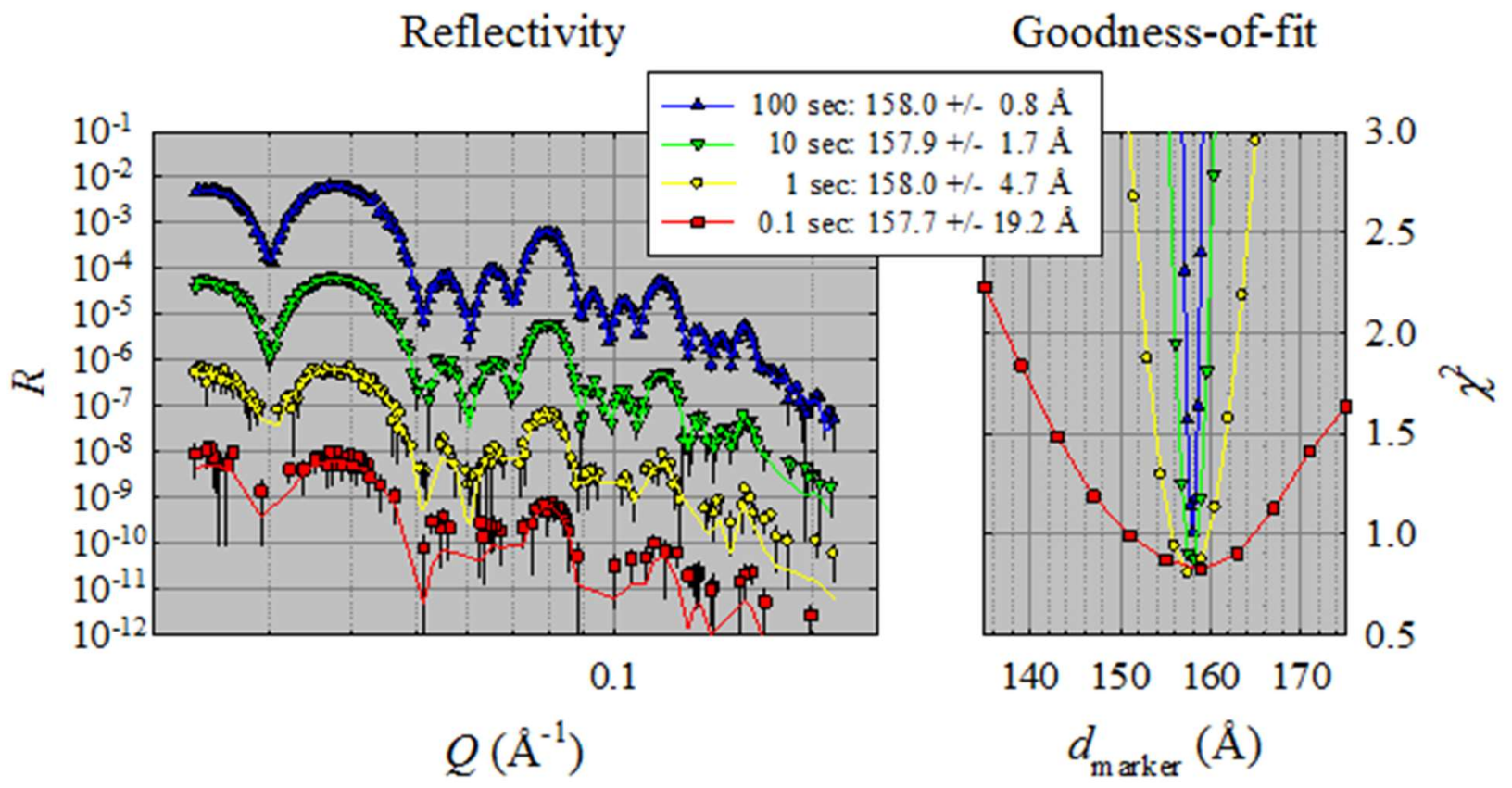

Figure 3.21. Simulated QIKR data for determining film thickness during EDA crosslinking • 
Collapse of the film during EDA crosslinking and PVPON release implies that the reaction can be well tracked by measuring the spacing between deuterated dPMAA marker layers. We can use the asdeposited data set shown in Figure 3.20B above and simulate the sensitivity of QIKR to changes in this spacing. Taking the fitted reflectivity curve from Figure 3.20B and using it to generate simulated data, we can then fit the simulated data and evaluate the dependence of $\chi^{2}$ on the bilayer spacing for different measurement times. As seen below, one can achieve a fair measure of film thickness already using a single neutron pulse $(0.1 \mathrm{sec})$ and a quite good measure in $1 \mathrm{sec}$. In situ kinetic measurements of this process are quite feasible. 


\subsection{A SMALL-/WIDE-ANGLE NEUTRON SCATTERING INSTRUMENT AT STS}

Scientists creating new and novel materials with features spanning a wide range of length scales, such as in the polymer and nanoscience communities, can greatly benefit from access to neutron scattering facilities and instrumentation suited to studying structures across length scales spanning from $\AA$ to $100 \mathrm{~s}$ of nm. As indicated in the Second Target Station Workshop Report on Instrumentation Needs that was developed after a 2015 meeting at ORNL, the three-source strategy set forward by ORNL requires coordination and optimization of suites of instruments at the ORNL neutron scattering facilities. HFIR was identified as the best place for traditional small-angle neutron scattering (SANS) instrumentation due to the high, continuous flux of the long wavelength neutrons needed to reach the lowest possible Q. However, the STS, which will operate at a lower frequency than the FTS, presents an opportunity to expand the suite of instruments for studying nanoscale materials with instruments that enable new science that currently requires making measurements on multiple instruments. A conceptual idea for an instrument for performing simultaneous small- and wide-angle neutron scattering (SWANS) was presented to the user community at the workshop and was recommended for the STS [1].

Key desired performance features called out in the workshop report for a SWANS instrument are:

- $\quad$ Small sample size $(1-100 \mu \mathrm{L})$

- Single shot Q-range spanning $0.01-6 \AA^{-1}$

- $\mathrm{dQ} / \mathrm{Q}$ of $1 \%$

Based on the workshop report, an initial concept of the instrument was developed, which is described herein, and its performance was estimated.

The general layout for the components of the SWANS instrument concept are presented in Figure 3.22 and is described in detail below. In summary, the SWANS instrument consists of:

- A $3 \mathrm{~cm}$ by $3 \mathrm{~cm}$ beam tube with a frame overlap mirror to remove long wavelength neutrons (>30 $\AA$ )

- A T0 chopper near the target shielding

- Straight $3 \mathrm{~cm}$ by $3 \mathrm{~cm}$ guides extending to $25 \mathrm{~m}$ from the source

- Two bandwidth choppers located at $10 \mathrm{~m}$ and $15 \mathrm{~m}$ from the source

- A source aperture and evacuated flight tube extending from $25 \mathrm{~m}$ to $30 \mathrm{~m}$ from the source

- A 0.5 m-wide open sample area

- A 5m-long evacuated detector tank with a low-Q bank $35.25 \mathrm{~m}$ from the source, and mid-Q banks at $32.25 \mathrm{~m}$ from the source

- Backscattering detector banks for high-Q that are $1 \mathrm{~m}$ from the sample position 


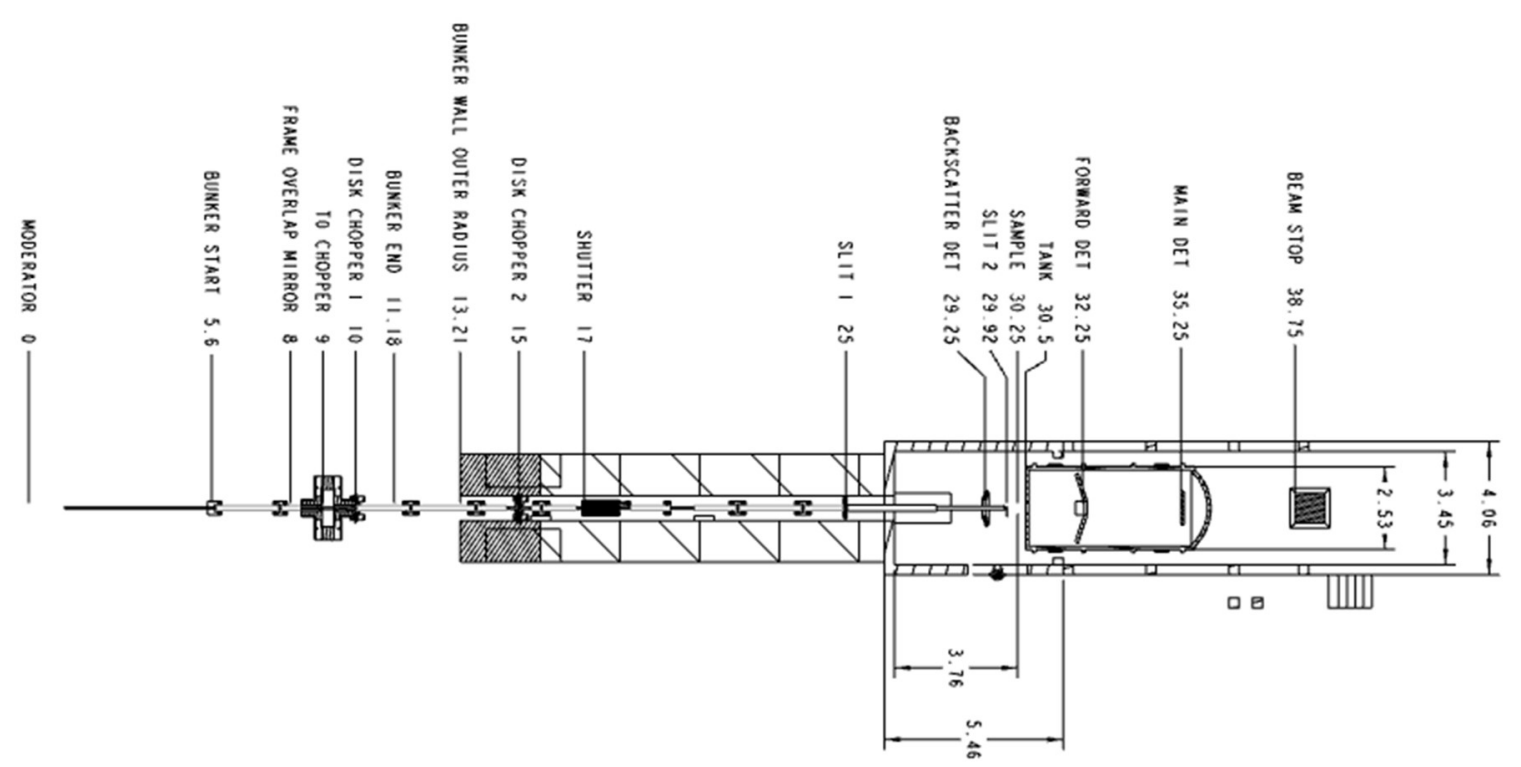

Figure 3.22. Schematic of the layout envisioned for the components of the SWANS instrument.

\section{The Source}

The source (i.e. moderator) that the SWANS instrument views impacts the design of the instrument. There are two possibilities for the moderator that the instrument views: a coupled supercritical parahydrogen moderator and a decoupled para-hydrogen supercritical moderator. In addition to having quite different spectra, these moderators have very different pulse shapes that impact the uncertainty in the wavelength-dependent time of emission of the neutron from the moderator, and therefore the best dQ/Q that can be achieved.

The peak brightnesses of the two aforementioned moderators are presented in Figure 3.23 (left). The data are from simulations performed by the SNS neutronics team. As can be seen, the decoupled moderator has a great deal less flux in the region of the spectrum above $0.5 \AA$, which are wavelengths that provide the low $\mathrm{Q}\left(<0.01 \AA^{-1}\right)$ that is required for studying large structures. The moderator type also determines the wavelength-dependent uncertainty in the emission time from the moderator. The wavelengthdependent FWHM of the emission times from the moderators are presented in Figure 3.23 (middle). The resulting relative uncertainty in the neutron time-of-flight for the coupled moderator is also presented in Figure 3.23 (right), while that of the decoupled moderator is much lower and therefore is not presented. The $\mathrm{dt} / \mathrm{t}$ for the coupled moderator peaks in a region centered around $2 \AA$, which is the region of the spectrum where the coupled moderator provides the greatest improvement in flux over the decoupled moderator. The scientific potential of time-resolved studies makes selecting the coupled moderator the preferred choice of source for the SWANS instrument, even at the expense of dQ/Q 

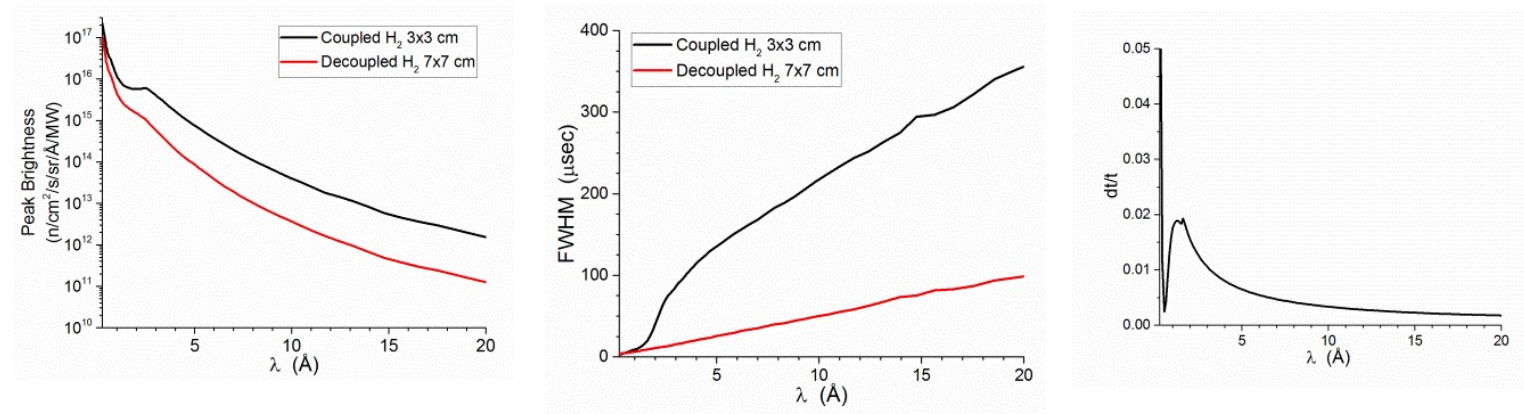

Figure 3.23. (left) Peak brightness of the two possible moderators that the SWANS instrument can view.

(middle) FWHM of the emission time from the moderator as a function of wavelength. (right) Uncertainty in the time-of-flight of a neutron as a function of wavelength for the coupled moderator.

\section{Neutron Delivery}

The neutron delivery system includes the monolith insert, frame-overlap mirror and guides. The monolith insert provides a low neutron absorption environment between the moderator face and the primary shutter. It is anticipated to be either filled with helium, or it will be evacuated. A frame-overlap mirror is required to remove long wavelength neutrons and will be mounted inside the core insert at the far end away from the moderator to ensure a long operating life. It will be a simple design, consisting of one or more sheets of thin fused silica or single crystal silicon set at an angle to prevent passage of neutrons longer than $30 \AA$.

The guides that transport the beam to the sample position

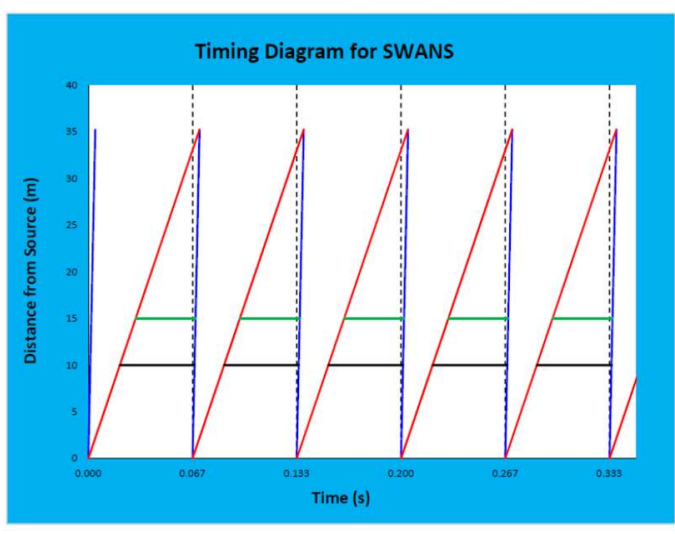

Figure 3.24. Chopper timing diagram. will be straight to ensure the best possible transmission of short wavelengths. In light of the compact moderators envisioned for the STS, the cross-sectional area of the guide at the entry just outside the primary shutter will be $3 \mathrm{~cm}$ by $3 \mathrm{~cm}$ to best match the source size. During detailed instrument design and optimization, a gently converging guide system will be considered for reducing the beam size at the sample position improve performance for small sample volumes, as was deemed desirable by the 2015 meeting attendees, most of whom were from the SANS community. The SWANS, being a hybrid instrument, will require additional community input regarding this design detail.

\section{The Chopper Systems}

The chopper system consists of a T0 chopper near the primary shutter and a pair of bandwidth choppers located downstream. The T0 chopper will be required to remove the prompt pulse and fast neutrons, which would otherwise be visible at the detectors due to the straight guide system. A design typical of those employed on the FTS of the SNS will be considered as a starting point. The T0 chopper will operate strictly at the source frequency of the STS to ensure that all prompt pulses are blocked. The two bandwidth choppers will be single disk choppers of a design similar to others employed at the facility. Figure 3.25 shows the timing diagram for the SWANS operating at $15 \mathrm{~Hz}$. It is not anticipated that the instrument will be operated at $7.5 \mathrm{~Hz}$, but the option will be explored during formal instrument design. The chopper positions do a very effective job at minimizing leakage of neutrons from undesired 
wavelengths. The first possible long-wavelength opening for neutrons created at $\mathrm{T}=0$ takes place after $\mathrm{T}$ $=0.200$ at the $15 \mathrm{~m}$ chopper position. Such long wavelength neutrons will be removed from the beam by the frame-overlap mirror (see Neutron Delivery, above) prior to the first chopper.

\section{Pre-sample Collimation}

The pre-sample collimation for the SWANS will be simple. Rather than a series of removable collimation sections for varying the source-to-sample distance, the instrument will have fixed collimation upstream of the sample position. The aim is to match the collimation to the detector geometry, which will also be fixed. At the end of the neutron delivery system, which is $25 \mathrm{~m}$ from the moderator surface, a final round aperture with the same diameter as the final crosssection of the neutron guide at that point will define the source. After the source aperture, the space between it and the start of the sample area will be an evacuated volume having a final sapphire or fused silica window. The beam at the sample position will be defined using round apertures anticipated to range from $1 \mathrm{~mm}$ to $10 \mathrm{~mm}$ in diameter.

\section{Sample Area}

The sample area for the SWANS instrument is $0.5 \mathrm{~m}$ along the neutron beam direction. The goal is to provide an open, versatile area that enables the use of complex sample environments, such as highpressure cells and magnetic fields. Equally important will be the development of sample environments that allow other in situ characterizations of the sample materials being studied by the SWANS, such as optical spectroscopies or x-ray scattering techniques. The only fixed element anticipated to be in the sample area is a positioning system capable of handling large loads, such as high-field magnets.

\section{Neutron Detection}

The neutron detection system will consist of multiple arrays of detectors using the current best technology for the range of wavelengths envisioned to make up the primary use cases for the SWANS, namely a minimum of $0.5 \AA$ up to a maximum on the order of $20 \AA$ when the minimum wavelength is set above the typical of $0.5 \AA$. The detector array consists of three banks: a low-Q detector placed $35.25 \mathrm{~m}$ from the source, an array of mid-Q detectors surrounding the angular range covered by the low-Q detector out to a maximum scattering angle of $30^{\circ}$, and a high-Q detector array that spans angles from $150^{\circ}$ to $165^{\circ}$, which is consistent with a backwards scattering direction and will be referred to as the backscattering array hereafter. The angular coverage is shown in Figure 3.25. The most important feature of the detector arrangement is the range of angles between $30^{\circ}$ and $150^{\circ}$ that are not covered by detectors. The gap in angular coverage enables the use of complex sample environments while the combination of high and low-angle detectors with the relatively broad wavelength band preserves the ability to measure the complete Q-range of the instrument.

\section{Expected Performance}

Given the aforementioned components of the SWANS, the available wavelength bandwidth and associated Q-ranges for the forward and backward scattering detector banks are resented in Table 3.5 for the $15 \mathrm{~Hz}$ frequency of the STS. The available bandwidth is dictated by the distance of the low-angle detector bank from the source. 
To estimate the flux on sample as a function of wavelength, we assume that the entrance to the guide is considered to be 1 $\mathrm{m}$ from the surface of the moderator. The guide is assumed to be straight, $3 \times 3 \mathrm{~cm}^{2}$, and the total transmission down the guide system is $50 \%$. The time-averaged flux on sample as a function of wavelength is shown in the upper graph in Figure 3.26. For the calculation, the facility is operating at $15 \mathrm{~Hz}$, which corresponds to $0.70 \mathrm{MW}$. The integrated flux on sample is $4.7 \mathrm{E}+07 \mathrm{n} / \mathrm{cm}^{2} / \mathrm{s}$. For comparison when the velocity selectors are set to $\Delta \lambda / \lambda=0.14$, similar configurations of GP-SANS $(4.75 \AA / 6$ guides $=2.0 \mathrm{E}+07$ $\mathrm{n} / \mathrm{cm}^{2} / \mathrm{s} ; 4.75 \AA / 7$ guides $\left.=3.5 \mathrm{E}+07 \mathrm{n} / \mathrm{cm}^{2} / \mathrm{s}\right)$ and Bio-SANS $\left(6 \AA / 6\right.$ guides $=7.1 \mathrm{E}+06 \mathrm{n} / \mathrm{cm}^{2} / \mathrm{s} ; 6 \AA / 7$ guides $=1.2 \mathrm{E}+07 \mathrm{n} / \mathrm{cm}^{2} / \mathrm{s}$ ) of HFIR have comparable fluxes, but the SWANS provides a lower Q-min and higher Q-max, even when only considering the forward scattering detector banks.
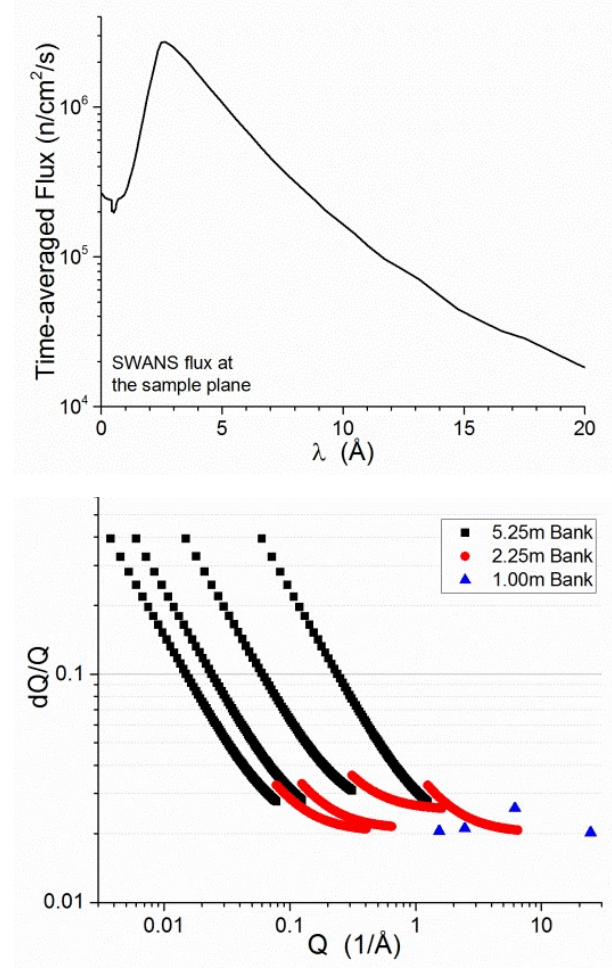

Figure 3.26. (top) Estimated flux on sample for the SWANS as a function of wavelength. (bottom) SWANS resolution, $\mathrm{dQ} / \mathrm{Q}$ as a function of $Q$.
Table 3.5. Bandwidth of neutron wavelengths and associated Q-ranges for the detector banks on the SWANS.

\begin{tabular}{ll}
\hline & \multicolumn{1}{c}{$\mathbf{1 5 ~ H z}$} \\
\hline Minimum $\boldsymbol{\lambda}(\AA)$ & 0.5 \\
Maximum $\boldsymbol{\lambda}(\AA)$ & 7.98 \\
Forward Q-range $\left(\AA^{-1}\right)$ & $0.0039-6.5048$ \\
Backward Scattering Q-range $\left(\AA^{-1}\right)$ & $1.5207-24.9177$ \\
\hline
\end{tabular}




\subsection{A SMALL-ANGLE NEUTRON SCATTERING INSTRUMENT AT STS}

As indicated in the Second Target Station workshop report produced after the 2015 meeting at ORNL,[1] the three source strategy set forward by ORNL requires coordination and optimization of suites of instrumentation across the ORNL neutron scattering facilities. The report identified HFIR as the best place for small-angle neutron scattering (SANS) instrumentation due to the high, continuous flux of long wavelength neutrons needed to reach the lowest possible Q. However, the STS source parameters resulting from the low operating frequency and high brightness moderators present an opportunity to expand the existing suite of SANS instruments. The SANS instrument presented below provides both a uniquely broad simultaneous Q-range and high flux that complements the existing suite of instruments at ORNL by enabling time-resolved studies of materials not readily addressed at present.

ORNL has three SANS instruments: the GP-SANS and Bio-SANS at HFIR and the EQ-SANS at the FTS of SNS. The instruments benefit from the high flux of their respective sources and have well-established user communities that creates a very strong demand for beam time. While the STS instrumentation workshop report called out upgrading the instruments at HFIR as a priority over the construction of new SANS at the STS, the STS is well-suited for a SANS instrument with capabilities that complement the existing suite of instruments. Another benefit of constructing a SANS beamline at the STS would be an increase in overall capacity for experiments, which would enable further user community growth due to greater availability of beam time. Presented here is a conceptual design for a general-purpose SANS instrument at STS that provides an indication of the performance possible. The SANS instrument is schematically shown in Figure 3.27. Briefly, it consists of the following elements.

- A monolith insert that brings the $3 \mathrm{~cm}$ by $3 \mathrm{~cm}$ guide to $1 \mathrm{~m}$ from the surface of the supercritical coupled moderator.

- A frame-overlap mirror to remove neutrons with wavelengths greater than $30 \AA$.

- The first double-disk chopper $6.2 \mathrm{~m}$ from the moderator

- A $3.3 \mathrm{~m}$ long bender with a radius of $95 \mathrm{~m}$

- $\quad$ The second double-disk chopper $9.8 \mathrm{~m}$ from the moderator

- Guides to a $1 \mathrm{~m}$ long optical component box starting $14 \mathrm{~m}$ from the moderator

- Variable collimation (two sections of $3 \mathrm{~m}$ guides with source apertures and a $2 \mathrm{~m}$ gap to the sample area)

- A 2 m sample area

- An evacuated detector tank with banks $1.3 \mathrm{~m}$ inside the tank, $2.5 \mathrm{~m}$ inside the tank and a moving low$\mathrm{Q}$ detector that can translate from $4 \mathrm{~m}$ to $10 \mathrm{~m}$ inside the tank 


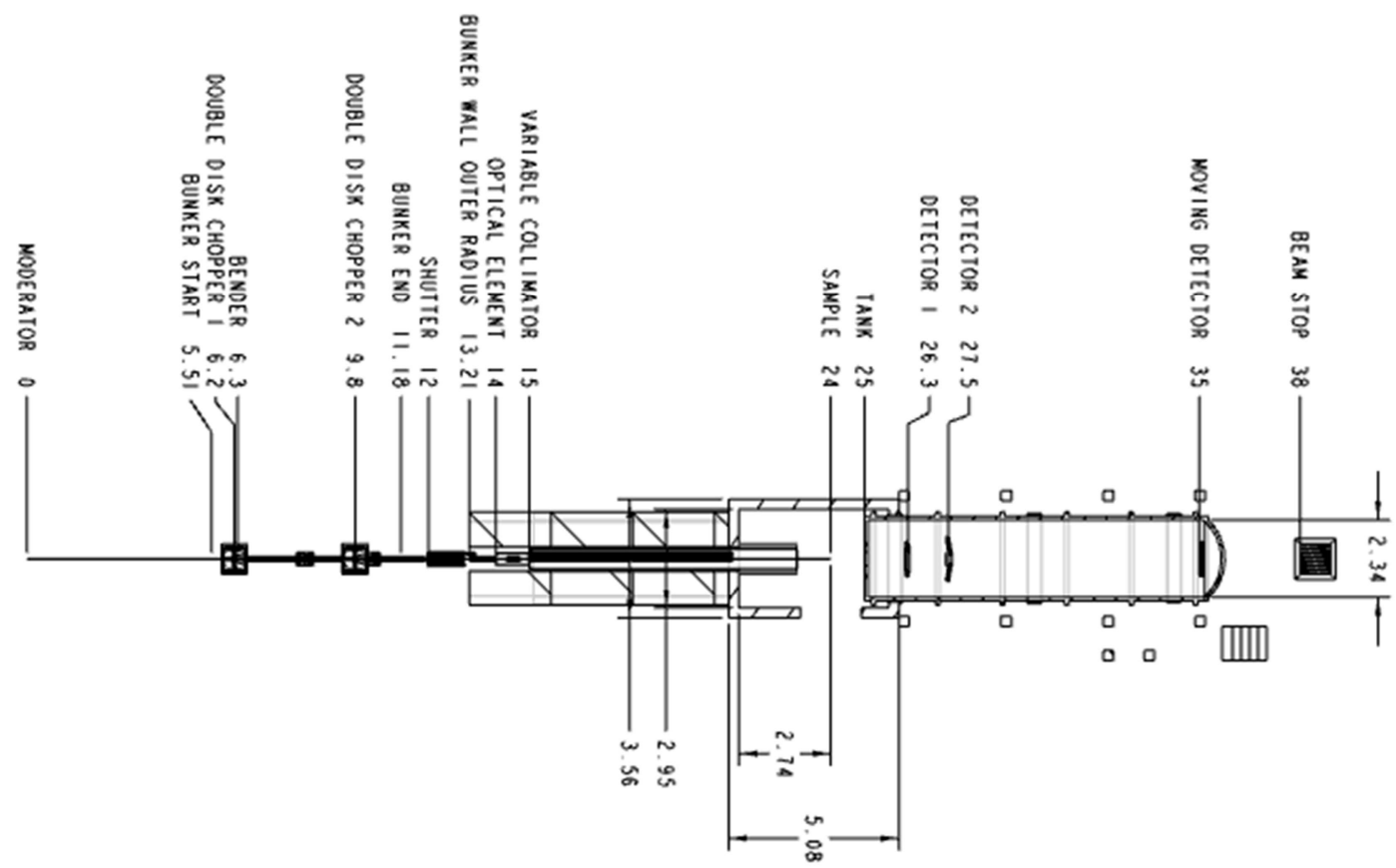

Figure 3.27. Schematic of the concept SANS instrument for STS.

\section{The Source}

The only logical choice for a moderator for a SANS instrument at the STS is the coupled para-hydrogen supercritical moderator because SANS is often a flux-limited technique. The peak brightness of this moderator and the decoupled para-hydrogen supercritical moderator are presented in Figure 3.28 for comparison. While the decoupled moderator provides much sharper neutron pulses, the higher wavelength, and therefore Q, resolution that results comes at the expense of neutron brightness, which is more important for SANS. Long wavelength neutrons are required for probing large scales structures, and the high brightness of the STS will enable time-resolved studies not possible with reactor-based SANS or a SANS on a higher-repetition rate source, such as the EQ-SANS of the FTS.

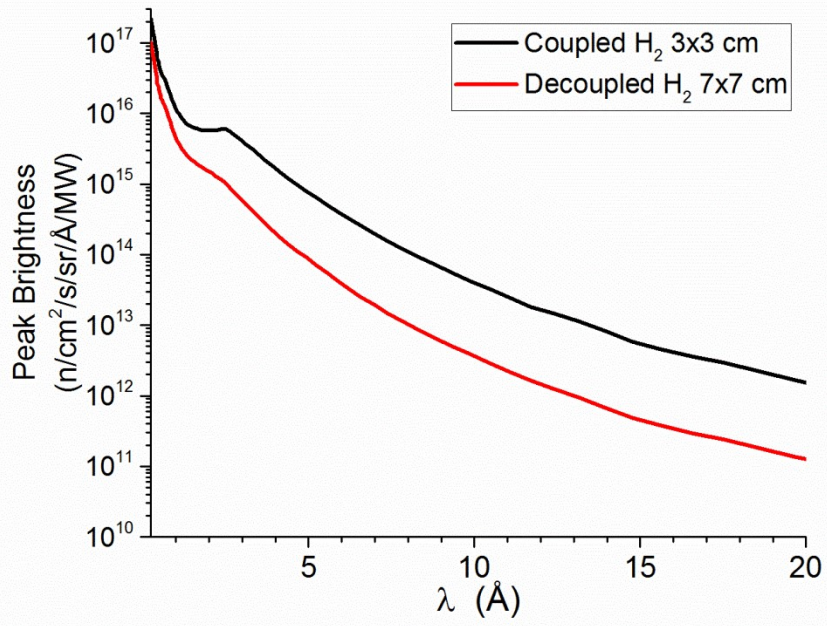

Figure 3.28. Brightness of the moderators that a SANS instrument can view.

\section{Neutron Delivery}

The neutron delivery system includes the monolith insert with frame-overlap mirror, a bender and guides. The monolith insert, which will include the frame-overlap mirror, provides a low neutron absorption environment between the moderator face and the maintenance shutter by either being evacuated or by 
being filled with helium, depending on cooling requirements. The monolith insert also includes a straight $3 \mathrm{~cm}$ by $3 \mathrm{~cm}$ guide that begins $1 \mathrm{~m}$ from the face of the moderator. The frame-overlap mirror removes long wavelength neutrons from the beam and will consist of either fused silica or single crystal silicon set at an angle that prevents passage of neutrons with wavelengths longer than $30 \AA$. The remainder of the neutron delivery system consists of straight $3 \mathrm{~cm}$ by $3 \mathrm{~cm}$ guides and a bender. The bender is $3.2 \mathrm{~m}$ long and has a radius of curvature of $95 \mathrm{~m}$. The bender is required to deflect the neutron beam from a direct line of sight of the moderator, which prevents the detector systems from receiving the prompt, fast neutron pulse and the associated gamma rays. As was demonstrated on EQ-SANS at FTS, a lower instrument background results without the added complexity of a T0 chopper upstream of the bandwidth choppers. All guides downstream of the bender are straight $3 \mathrm{~cm}$ by $3 \mathrm{~cm}$ guides.

\section{The Chopper System}

The chopper system consists of two double disk choppers that are located $6.2 \mathrm{~m}$ and $9.8 \mathrm{~m}$ from the face of the moderator. The possibility of changing the overall length of the instrument by moving the low-Q detector along the length of the tank necessitates the more versatile chopper design. Two example timing diagrams for the SANS instrument are presented in Figure 3.29: one for the maximum low-Q detector distance when operating at $15 \mathrm{~Hz}$, and one for the minimum low-Q detector distance when operating at $7.5 \mathrm{~Hz}$.

\section{Pre-sample Collimation}

The pre-sample collimation of the SANS instrument shares design features found on reactor-based SANS. At $14 \mathrm{~m}$ from the source, the instrument has a $1 \mathrm{~m}$ box into which optical elements can be installed for enabling specific science. Possibilities include lenses, a neutron polarizer or a chopper to define a narrow wavelength band. When no optical element is in place, the gap will be filled with an evacuated section of guide consistent with the guides in the remainder of the instrument.

After the optical element box, the instrument has variable collimation similar to that employed on reactor-based SANS. Two sections of removable guide that are $3 \mathrm{~m}$ long will be in an evacuated chamber. When a guide section is translated out of the beam, the beam position is either open, or a source aperture is in place to define the incident beam. After the guides, there is a $2 \mathrm{~m}$ section that is normally empty, but will include positioning
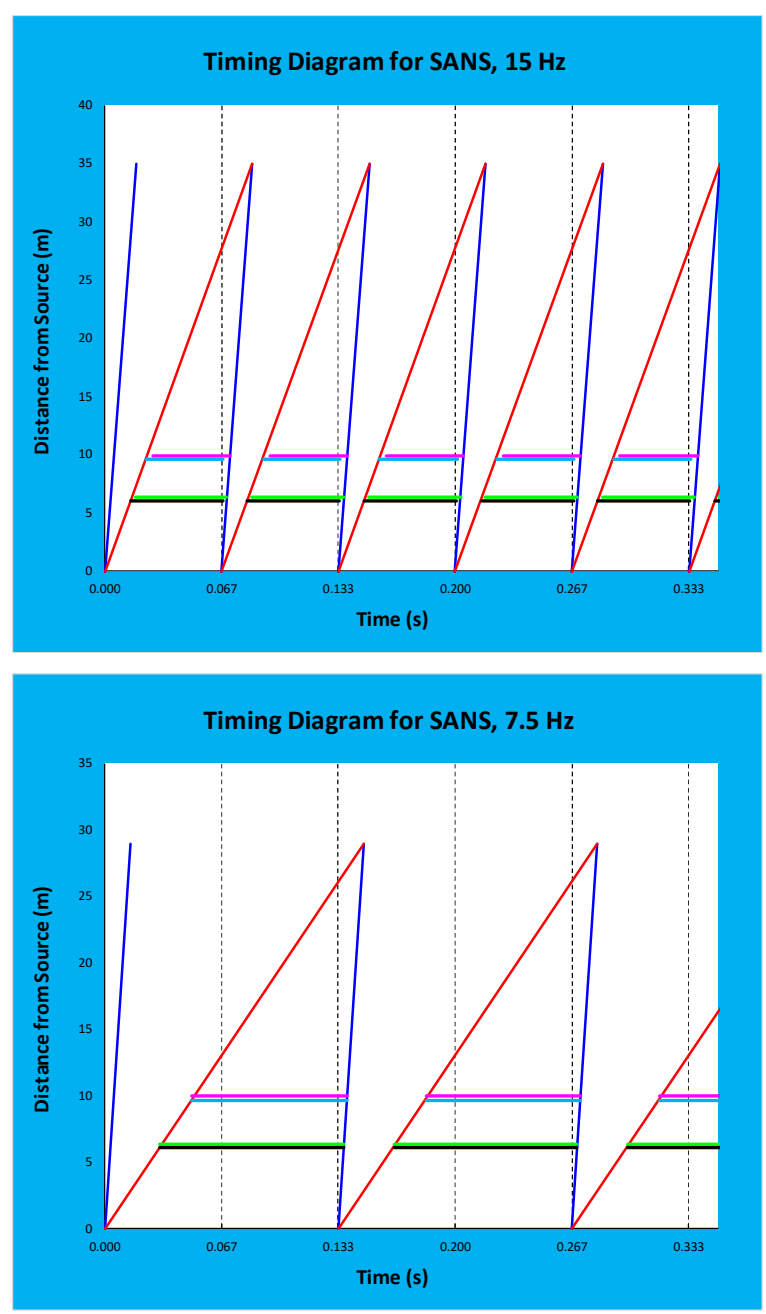

Figure 3.29. Chopper timing diagram for (top) low$Q$ detector at $10 \mathrm{~m}, 15 \mathrm{~Hz}$ operation and (bottom) low-Q detector at $4 \mathrm{~m}, 7.5 \mathrm{~Hz}$ operation. hardware for use with an optical element for beam conditioning. The variable collimation can then either provide source distances (to the upstream tank face) of $10 \mathrm{~m}, 7 \mathrm{~m}$ or $4 \mathrm{~m}$. After this fixed section, the instrument has a neutron transparent window of either sapphire or fused silica that can be mounted on a vacuum extension, which helps minimize 
background due to air scattering. Also mounted near the window are sample apertures, which are anticipated to be circular apertures with diameters ranging from $1 \mathrm{~mm}$ to $10 \mathrm{~mm}$.

\section{Sample Area}

The sample area for the SANS instrument is $2 \mathrm{~m}$ along the neutron beam direction. The goal is to provide an open, versatile area that enables the use of complex sample environments, such as high-pressure cells and high field magnets. Equally important to the long-term productivity of the instrument will be the development of sample environments that allow multi-modal and in situ characterizations of samples, such as optical spectroscopies and x-ray scattering techniques.

\section{Neutron Detection}

The neutron detection system will consist of multiple arrays of detectors that will use the best technology available at the time for the range of wavelengths envisioned to make up the primary use cases for the SWANS, namely a minimum of $2.0 \AA$ up to a maximum of $30 \AA$. The detector array consists of three banks: a $1 \mathrm{~m}$ by $1 \mathrm{~m}$ low-Q detector placed that can move from $4 \mathrm{~m}$ to $10 \mathrm{~m}$ from the upstream face of the detector tank, an array of mid-Q detectors set $2.5 \mathrm{~m}$ from the upstream face of the detector tank that span the angular range from $2.79^{\circ}<2 \theta<15^{\circ}$ and a high-Q detector array set $1.3 \mathrm{~m}$ from the upstream face of the detector tank that covers from $15^{\circ}<2 \theta<22.5^{\circ}$. Angular coverage will be both horizontal and vertical to make the instrument suitable for studies of anisotropic materials. One of the key advantages of a timeof-flight source is the ability to reach a relatively high Q-max without needing to measure to large scattering angles. The reduced angular range required makes performing complete Q-range measurements with complex and robust sample environments easier. It is anticipated that the detectors will be ${ }^{3} \mathrm{He}$ with pixel dimensions on the order of $5 \mathrm{~mm}$, but next generation detector technologies will be leveraged, if they are suitable and cost effective for SANS.

\section{Expected Performance}

The wavelength bandwidths and associated Q-ranges for the SANS operating with the choppers rotating at $15 \mathrm{~Hz}$ mode, as well as one example calculated assuming $7.5 \mathrm{~Hz}$ operation of the choppers, are presented in Table 3.6. The available bandwidth is dictated by the distance of the low-Q detector from the moderator. The dynamic range for the instrument is excellent.

Table 3.6. Bandwidth of neutrons, associated Q-ranges and integrated flux on sample.

\begin{tabular}{|c|c|c|c|c|c|}
\hline & $\begin{array}{c}15 \mathrm{~Hz}, 10 \mathrm{~m} \\
\text { (no guides) }\end{array}$ & $\begin{array}{l}15 \mathrm{~Hz}, 10 \mathrm{~m} \\
\text { (no guides) }\end{array}$ & $\begin{array}{l}15 \mathrm{~Hz}, 7 \mathrm{~m} \\
\text { (1 guide) }\end{array}$ & $\begin{array}{l}15 \mathrm{~Hz}, 4 \mathrm{~m} \\
\text { (2 guides) }\end{array}$ & $\begin{array}{c}7.5 \mathrm{~Hz}, 10 \mathrm{~m} \\
\text { (no guides) }\end{array}$ \\
\hline Minimum $\lambda(\AA ̊)$ & 2.00 & 10.00 & 2.00 & 2.00 & 2.00 \\
\hline $\operatorname{Minimum} \lambda(\AA ̊)$ & 9.54 & 17.54 & 10.24 & 11.09 & 17.07 \\
\hline Q-min $\left(\AA^{-1}\right)$ & 0.0017 & 0.0009 & 0.0022 & 0.0037 & 0.0009 \\
\hline Q-max $\left(\AA^{-1}\right)$ & 1.2258 & 0.2452 & 1.2258 & 1.2258 & 1.2258 \\
\hline $\begin{array}{l}\text { Integrated Flux } \\
(\mathrm{n} / \mathrm{cm} 2 / \mathrm{s})\end{array}$ & $1.1 \mathrm{E}+07$ & $2.1 \mathrm{E}+05$ & $2.2 \mathrm{E}+07$ & $6.4 \mathrm{e}+07$ & $5.5 \mathrm{E}+06$ \\
\hline
\end{tabular}

The flux of the instrument as a function of wavelength was estimated by assuming that the entrance to the $3 \mathrm{~cm}$ by $3 \mathrm{~cm}$ guide is $1 \mathrm{~m}$ from the surface of the moderator and that the total transmission down the guide system is $50 \%$, which is a reasonable approximation pending development of an optimized guide design. The estimated flux on sample as a function of wavelength for the three possible guide configurations presented in Table 3.6 are shown in Figure 3.30 and the integrated fluxes are presented in Table 3.6. For comparison with comparable collimation, the Bio-SANS and GP-SANS of HFIR have 
$\sim 2.5 \mathrm{E}+06 \mathrm{n} / \mathrm{cm}^{2} / \mathrm{s}$ and $\sim 7.9 \mathrm{E}+06 \mathrm{n} / \mathrm{cm}^{2} / \mathrm{s}$ for 4 guides (column 4 of Table 3.6) and the shortest normal operating wavelengths $(6.0 \AA$ and $4.8 \AA$, respectively), which is equivalent to the no guides and short wavelength setting for the SANS described. Similarly, the Bio-SANS and GP-SANS of HFIR have $\sim 3.0 \mathrm{E}+04 \mathrm{n} / \mathrm{cm}^{2} / \mathrm{s}$ for 4 guides and the longest normal operating wavelength $(17.8 \AA$ and 19.0 $\AA$, respectively), which is equivalent to the no guides and long wavelength setting for the SANS described herein. The highest flux provided by the SANS described herein also compares favorably with those normally used on the two HFIR SANS when 7 guides are employed (equivalent to the 2 guides collimation case presented in Table 3.6), being $\sim 1.2 \mathrm{E}+07 \mathrm{n} / \mathrm{cm}^{2} / \mathrm{s}$ at $6 \AA$ for Bio-SANS and $\sim 2.0 \mathrm{E}+07 \mathrm{n} / \mathrm{cm}^{2} / \mathrm{s}$ for the GP-SANS at $4.8 \AA$. With the same minimum wavelength and low-Q detector setting, EQ-SANS has a flux of $1.3 \mathrm{E}+07 \mathrm{n} / \mathrm{cm}^{2} / \mathrm{s}$.

None of the current suite of SANS instruments at ORNL, including the EQ-SANS at FTS, cover as broad of a Q-range at a single instrument configuration as this proposed SANS instrument.

The Q-resolution of the instrument at low scattering angles is dominated by the uncertainty in the angles defined by the collimation and by the pixel size, while the high-Q resolution at higher scattering angles is dominated by the intrinsic uncertainty in the timeof-flight of the neutron, $\mathrm{dt} / \mathrm{t}$. The $\mathrm{dt} / \mathrm{t}$ for the high-q bank of detectors, which is $26.3 \mathrm{~m}$ from the source, is shown as a function of wavelength in Figure 3.31 (left) and never exceeds $3 \%$ in the range of wavelengths envisioned for normal instrument operation.

Example resulting dQ/Q is presented in Figure 3.31 (right). For the calculations, the wavelengths were binned to $0.1 \AA$, as is done for the EQ-SANS instrument at the FTS, which improves the speed of the data reduction process to an acceptable level of performance. For $2 \AA$ neutrons, this contribution to dQ/Q is $5 \%$, but it is negligible for $17.1 \AA$ neutrons where $\mathrm{dQ} / \mathrm{Q}$ is always dominated by the angular uncertainties.

\subsubsection{References}

[1] Second Target Station Workshop, Co-chairs: M. R. Eskildsen (University of Notre Dame) and B. Khaykovich (Massachusetts Institute of Technology), https://public.ornl.gov/conferences/neutrons/STS2015/index.shtml. 


\subsection{MENUS - MATERIALS ENGINEERING BY NEUTRON SCATTERING}

\subsubsection{Abstract}

MENUS-Materials Engineering by Neutron Scattering at the STS will be a transformational high flux versatile multi-scale materials engineering beamline with unprecedented new capabilities. Specifically, in the Q range optimized for low symmetry materials, MENUS has improved flux of up to 3 orders of magnitude higher than VULCAN at the SNS first target station can deliver. With large out-of-plane detector coverage, high spatial resolution residual/in-situ stress measurements can be performed rapidly at once and in-situ full ODF/SODF (orientation distribution function/stress-orientation distribution function) can be recorded by rotating the sample around a single axis. In addition, SANS and imaging capabilities are optimized for materials engineering. MENUS will provide neutron scattering data with high temporal and spatial resolution to the materials science and engineering community to tackle and understand lattice strain / phase transition / microstructure / texture evolution in three orthogonal directions in complex material systems under combined extreme conditions of stress, temperature, electric and/or magnetic fields, etc. It will open new scientific opportunities by using neutron scattering for material exploratory, material processing, and material operations, etc.

\subsubsection{Science case}

The wide Q range coverage in a single frame, the high flux at long wavelength and the novel detector layout make MENUS unique for studying complex structural and functional material behaviors under mechanical, thermal, electrical, and magnetic fields. The rapid data collection rate and the capability to handle large lattice cells or superlattice structures allow studying phenomena critical to manufacturing and operation of new advanced materials such as:

- Advancing the design of critical engineering materials with superlattice structure by understanding phase specific stress partitioning.

- Revealing the anisotropic behavior of low symmetry system.

- Understanding the underlying chemistry science in energy materials in operando.

- Decoupling the structural and magnetic behavior in functional materials under external extreme fields.

- Unraveling kinetic phase transformation in functional materials and alloys.

- Rapid deep strain scanning in engineering structures with high spatial resolution.

\section{Science Requirements}

Wide out-of-plane detector coverage to provide three orthogonal direction structural information simultaneously and large d-range coverage of diffraction lines under combined extreme fields; high spatial resolution detector for diffraction imaging; downstream detectors for simultaneous SANS.

Exchangeable guides design to satisfy different experimental needs of time resolved or spatially resolved measurements; open sample area to accommodate various sample environments.

Sub-second data collection rate and live data streaming/analysis protocol as required for in situ or in operando observations. 


\subsubsection{Conceptual Design}

\subsubsection{Sample size, beam divergence, wavelength range}

The MENUS instrument will view the high-peak-brightness coupled para-hydrogen moderator (HPCM-c) with a $3 \times 3 \mathrm{~cm}^{2}$ cross-section at a sample-moderator distance of about $72 \mathrm{~m}$. A single frame at $15 \mathrm{~Hz}$ will allow a bandwidth of $3.516 \AA$, which is comparable to the effective bandwidth in use at VULCAN (FTS) with $1 / 3$ frame suppression $(20 \mathrm{~Hz})$. The improved moderator brightness and the adequate repetition rate delivers a performance gain of three orders of magnitude in the $d$ spacing range over $2 \AA$. The maximum wavelength range expected to be used spans from 1 to $8 \AA$, at $7.5 \mathrm{~Hz}$ with $1 / 2$ frame suppression, which corresponds to d-spacing range of $0.5-7 \AA$ (or $0.9-12.5 \AA^{-1} \mathrm{Q}$ range) for the full detector coverage. At least two instrument configurations are envisioned: high intensity (HI) and high resolution (HR). The HI option will permit fine tuning of neutron beam divergence and beam size at the sample position in the range of $0.2^{0}-0.6^{0}$ and $1-5 \mathrm{~mm}$ diameter, respectively. HI configuration will be dedicated for in situ studies of materials under thermomechanical testing and residual stress mapping in complex engineering components. The HR option will provide a tight divergence of $0.12^{0}$ and a larger cross section of about 2 $\mathrm{cm}^{2}$. The HR option is expecting to add more flexibility for studies of functional materials under simultaneous application of stress, temperature, electric or magnetic fields. Moreover, this option will enable transmission and diffraction contrast neutron imaging, as well as simultaneous small angle neutron scattering (SANS) capability.

\subsubsection{MENUS optics design}

Figure 3.32 shows the MENUS optics design. An elliptic guide, $19 \mathrm{~m}$ long, with only two reflecting walls, deflects the neutron beam in both horizontal and vertical planes. The beam is focused next by a shorter $(6.5 \mathrm{~m})$ nested Kirkpatrick-Baez $(\mathrm{KB})$ mirror to a slit system defining an intense secondary neutron source of $10 \times 10 \mathrm{~mm}$ cross section and $0.3^{0}$ divergence. The deflection of the beam at the slit location is $230 \mathrm{~mm}$ in both horizontal and vertical plane, which ensures a substantial reduction of fast neutrons. Two neutron mirror systems are proposed to deliver the neutron beam at the sample position according to the requirements of HI and HR instrument configurations. For the HI option, a nested Wolter mirror type system, enclosing elliptic followed by hyperbolic shaped reflecting walls, focuses the neutrons at the sample position in a square area of maximum 5 by $5 \mathrm{~mm}^{2}$ and a beam divergence of $0.6^{0}$. The beam size at the sample position can be adjusted by changing the opening at the secondary source from the primary slit and the beam divergence can be controlled by tuning a slit (not shown) located at the end of the focusing mirror device. The full length of the device extends to about $7 \mathrm{~m}$. For the HR option, a similar Wolter mirror type system is envisioned containing hyperbolic followed by parabolic shaped reflecting walls. This arrangement provides a quasi-parallel beam with the beam divergence controlled by the secondary source extension and the cross section of the beam by the exit slit. A translation stage across the beam direction will allow quick switching between the two configurations. A SANS detector is positioned $5 \mathrm{~m}$ downstream from the sample positon and an insertable MCP detector will be just downstream of the sample position. 


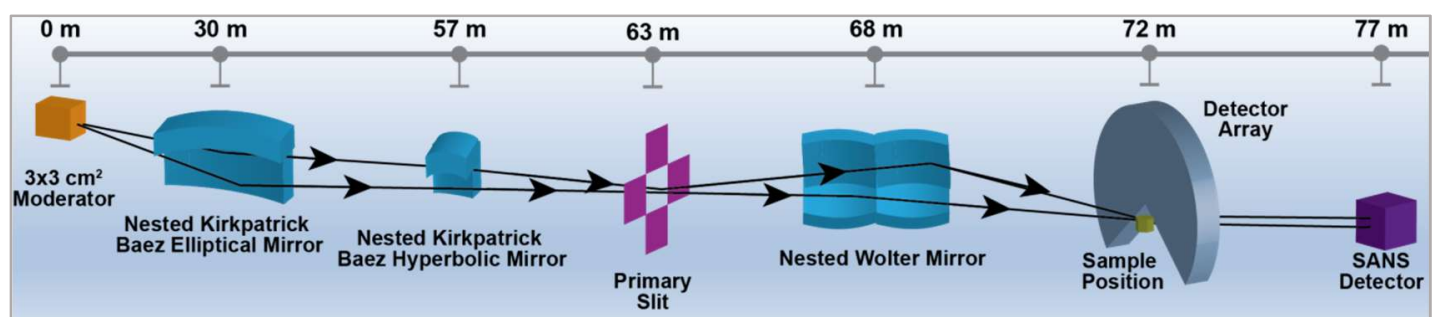

a)
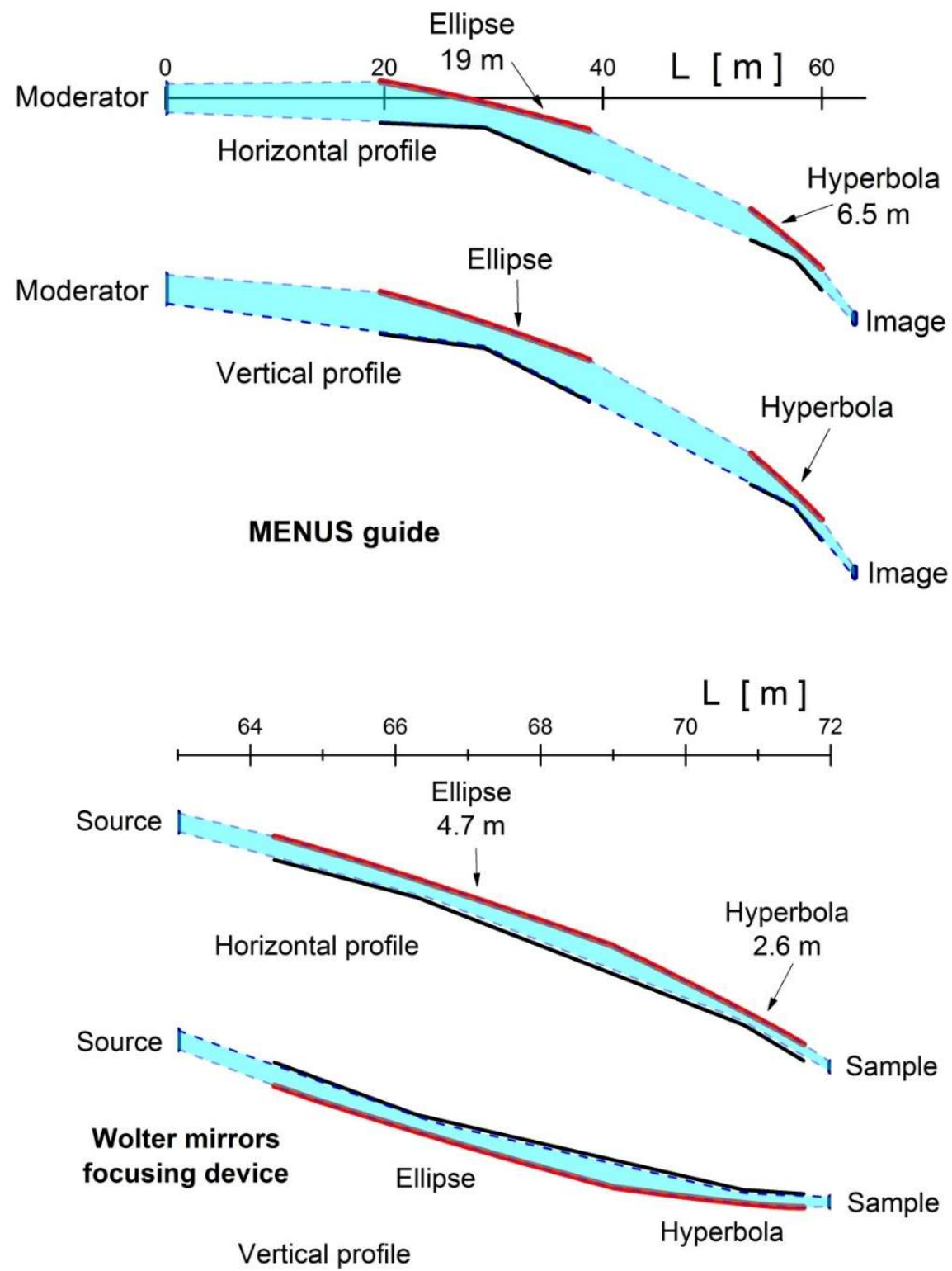

b)

Figure 3.32. The MENUS optics design, a) the schematic view, b) the optics details in horizontal and vertical directions. 


\subsubsection{Choppers and Timing}

The frame definition choppers functionality is determined by the total neutron flight path including moderator-sample $(72 \mathrm{~m})$ and sample-detector distance $(3 \mathrm{~m})$. The chopper timing graph is presented in Figure 3.33. The first chopper (double disk) is located at $6.5 \mathrm{~m}$ from the moderator and has the disk opening of about $34.7^{0}$. The double disk design provides a reduced opening time and allows for changing the wavelength bandwidth, which is a feature required by some special applications. Beside the working bandwidth, the first chopper transmits slow neutrons (of about $40 \AA$ ) to arrive at the detector location; to absorb these unwanted neutrons, a second chopper was placed at $10 \mathrm{~m}$ from the moderator, with a disk opening of $52^{\circ}$. A correlation chopper (not shown) with $50 \%$ duty cycle will be optional for providing super high resolution at the instrument.

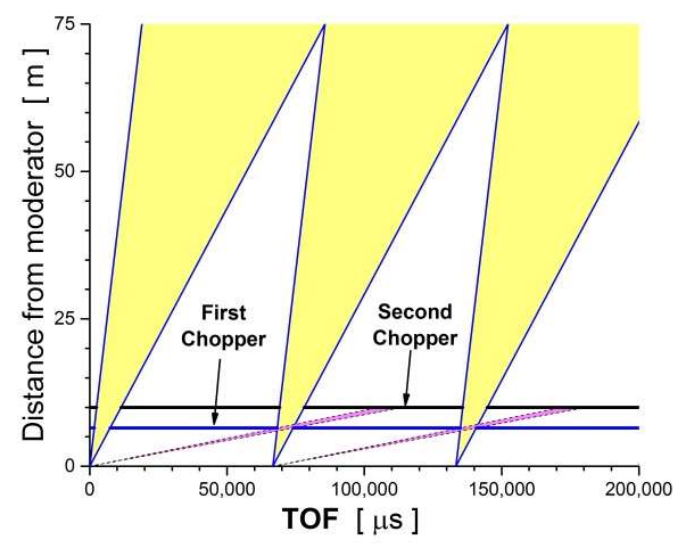

Figure 3.33. The MENUS timing diagram.

\subsubsection{Detector layout and type}

A tilted array of detectors by double layered $8 \mathrm{~mm} \mathrm{He} 3$ tube detector will be positioned at $70.5^{0}$ scattering angle (from $61^{\circ}$ to $80^{\circ}$ ) and will span around the emerging neutron beam direction inside a $250^{\circ}$ angular interval. Two other detector banks will be positioned around $+/-90^{\circ}$ scattering angles (from $80^{\circ}$ to $115^{\circ}$ ) and extend over about $48^{0}$ out of horizontal plane (from $-15^{0}$ to $33^{\circ}$ ). The coverage of the pole figure in the laboratory coordinates is presented in Figure 3.34a, as well as the pole figure in specimen coordinates, when the loading axis (LD) is tilted with $54.74^{\circ}$ away of vertical axis (Figure 3.34b). The proposed detector configuration allows to expedite the diffraction analysis by measuring non-coplanar components of the strain; moreover, a complete determination of the orientation distribution function (ODF) and stress orientation distribution function (SODF) in polycrystalline samples can be performed by simply rotating the sample around a single axis (maximum 13 steps around the tilted loading direction specified in Figure 3.34). An additional detector bank will be deployed in back-reflection from $150^{\circ}$ to $170^{\circ}$ and will be dedicated to measurements of high resolution in reciprocal space required by special material science problems when specific diffraction lines are too close to be separated in the usual configuration of the instrument. The SANS detector located at $5 \mathrm{~m}$ from the sample position will allow in-situ simultaneous diffraction and SANS measurements for kinetic phase transformation studies in functional materials and alloys. The HR set-up will include also include small high spatial resolution detectors (MCP type) for imaging in diffraction to visualize the defect distribution and the grain structure in single crystals and coarse polycrystals. 

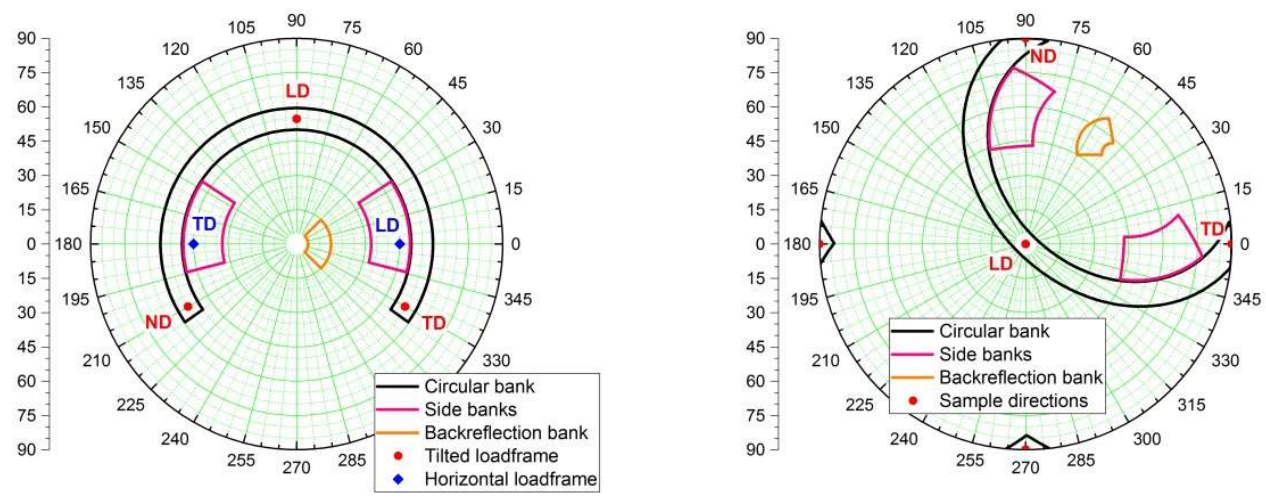

Figure 3.34. MENUS detector coverage. a) Detector coverage pole figure with beam at the center of the pole. and b) Detector coverage in sample pole figure with the loading direction in the center of the pole.

\subsubsection{Engineering model}

The conceptual design of MENUS is shown in Figure 3.35. MENUS will tackle problems beyond engineering materials and it will serve the community as a materials characterization lab with multi-probe capabilities including conventional probing techniques. As shown in the footprint of the instrument, the lab will be equipped with other tools with the capability to investigate radiated samples, such as lapping machines, microscopes, furnaces, thermogravimetric analysis systems, dynamic mechanical analysis devices, resonant ultrasound spectrometers, offline mechanical loadframes, etc.
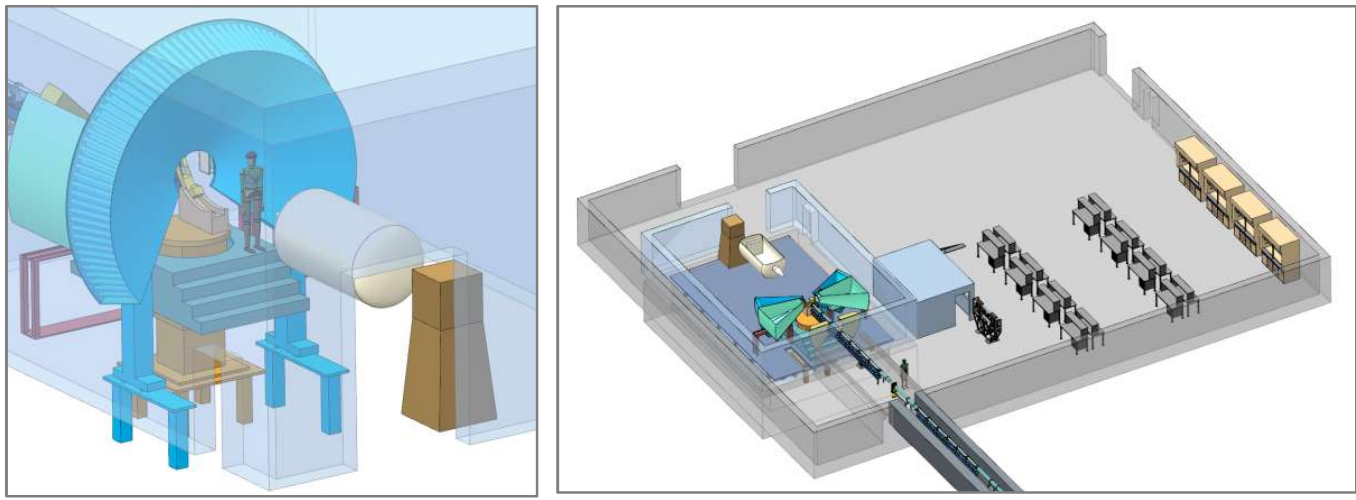

Figure 3.35. The conceptual design of multi-modal MENUS. a) with unique detector coverage and b) the materials lab footprint.

\subsubsection{MENUS performance estimation in flux and resolution}

MENUS performance is compared to VULCAN in terms of gain in flux as well as in diffraction spectra. As shown in Figure 3.36 a) as a benefit of the long pulse of STS, MENUS have significant gain in long wavelength in bandwidth and flux which peaks at $4.14 \times 10^{8} \mathrm{n} / \mathrm{cm}^{2} / \AA / \mathrm{s}$ at $\sim 3 \AA$ in $\mathrm{HI}$ mode. The simulated vanadium spectra gains with respect to different VULCAN operation modes show up to 3 orders or more of magnitude increase in count rate at lower Q region. The gain is phenomenal even if only the 90 degree banks are used. This allows measuring large lattice or superlattice peaks rapidly, as shown clearly by the gain in the first two peaks $(100,110)$ of the simulated $\mathrm{Ni}_{3} \mathrm{Al}$ diffraction patterns in Figure $3.36 \mathrm{c}$ ) and d) at large d-spacing where they are almost invisible in the VULCAN diffraction spectrum. The resolution of MENUS can be below the regime of $0.1 \%$ in super high resolution mode with an optional correlation 
chopper (Figure 3.37a). This allows tackling challenging materials engineering problem like misfit of a superalloy as evidenced by the simulation of gamma and gamma-prime phases in a Ni-based superalloy. The separation of (200) peaks from $110^{\circ}$ detector (as shown in Figure 3.37b) of $\mathrm{Ni}$ and $\mathrm{Ni}_{3} \mathrm{Al}$ along with the measurement of (100) superlattice peak of $\mathrm{Ni}_{3} \mathrm{Al}$ makes quantification of the misfit easier and more accurate.

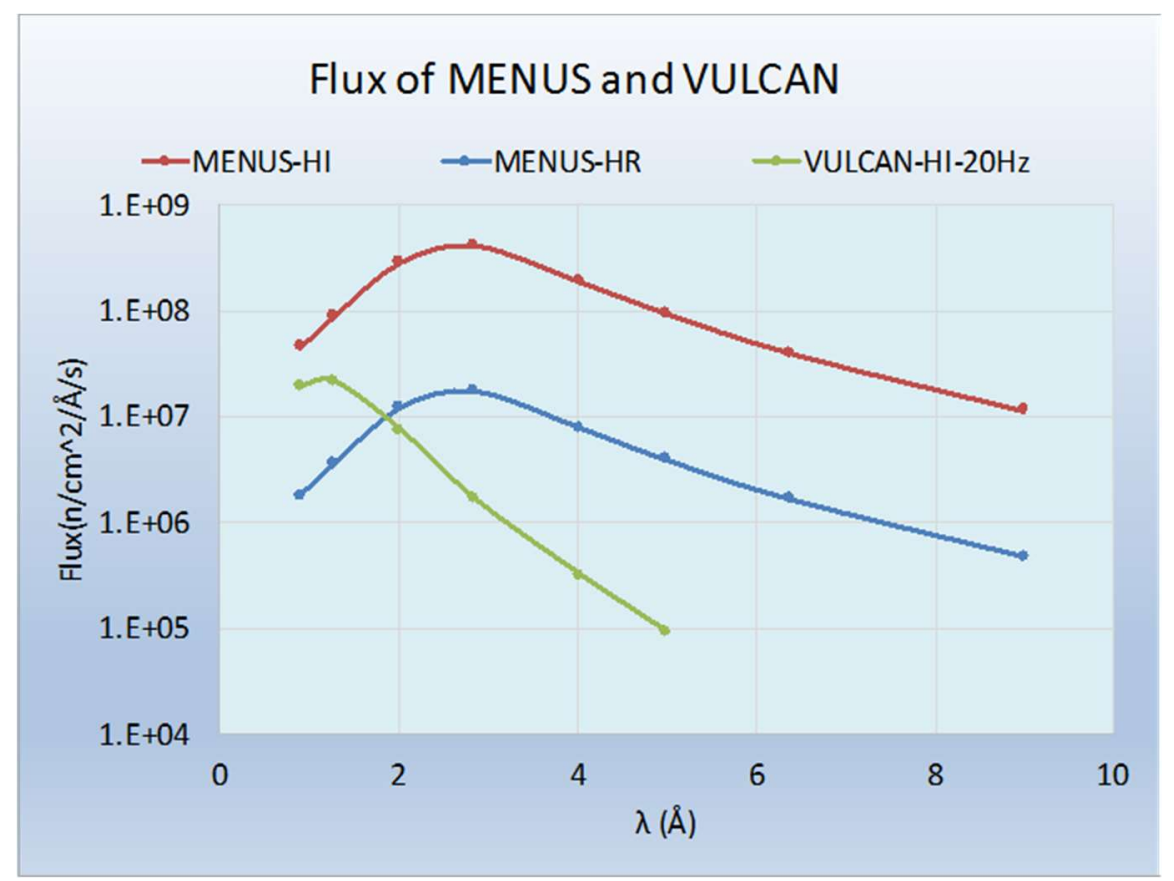

a)

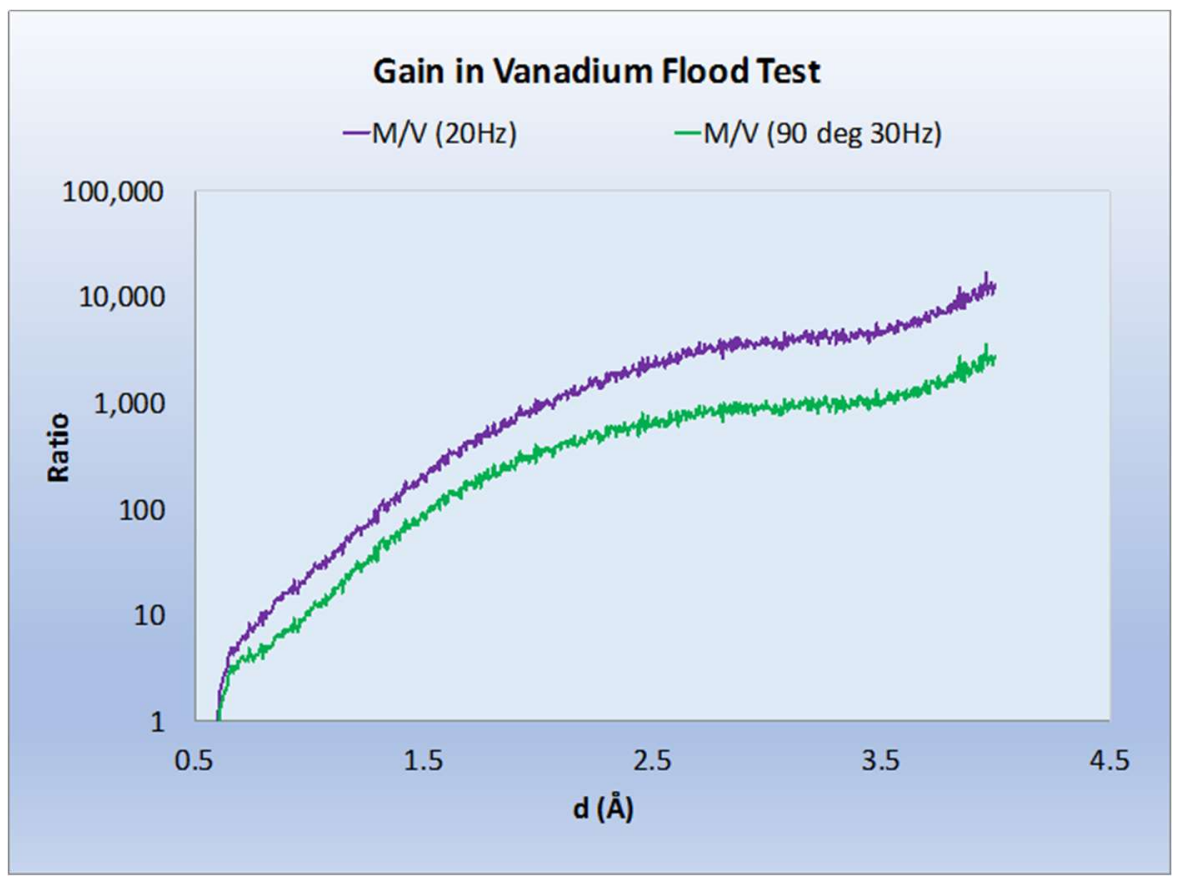

b) 


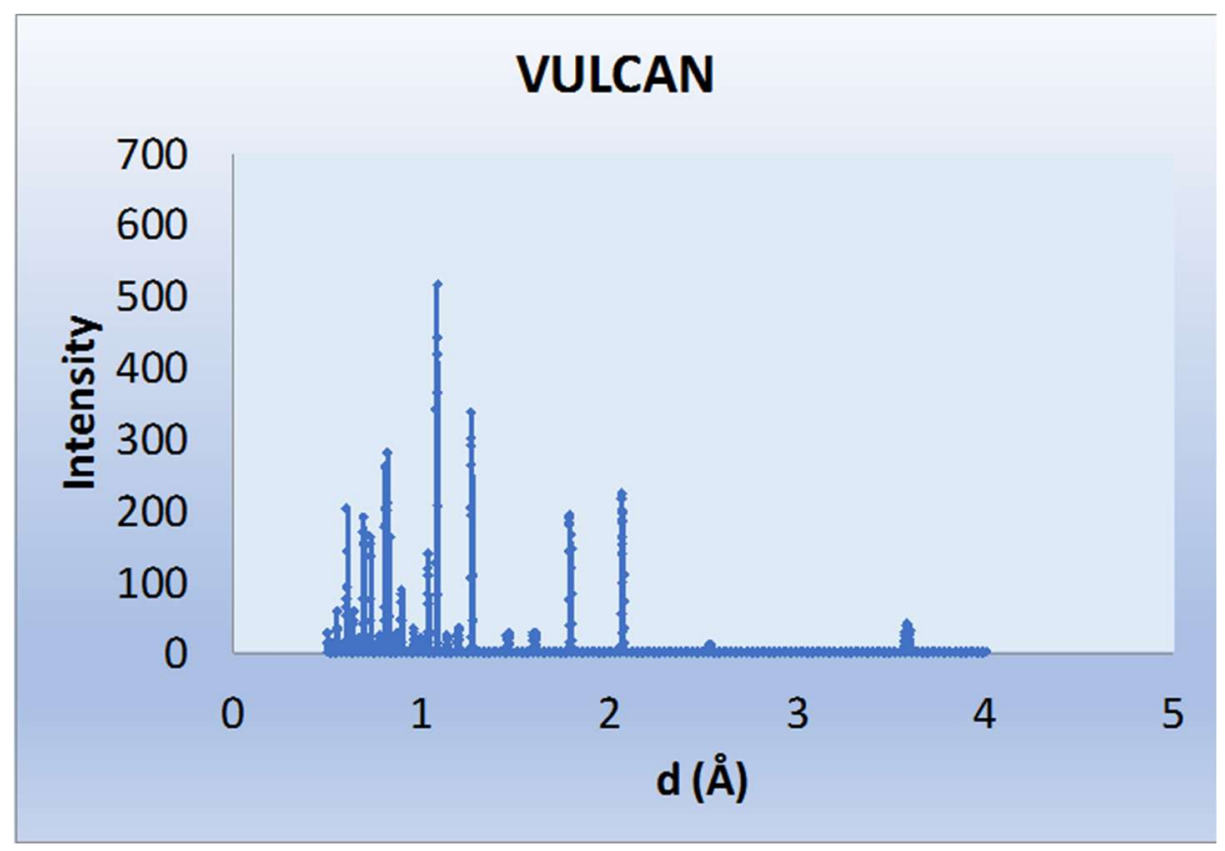

c)

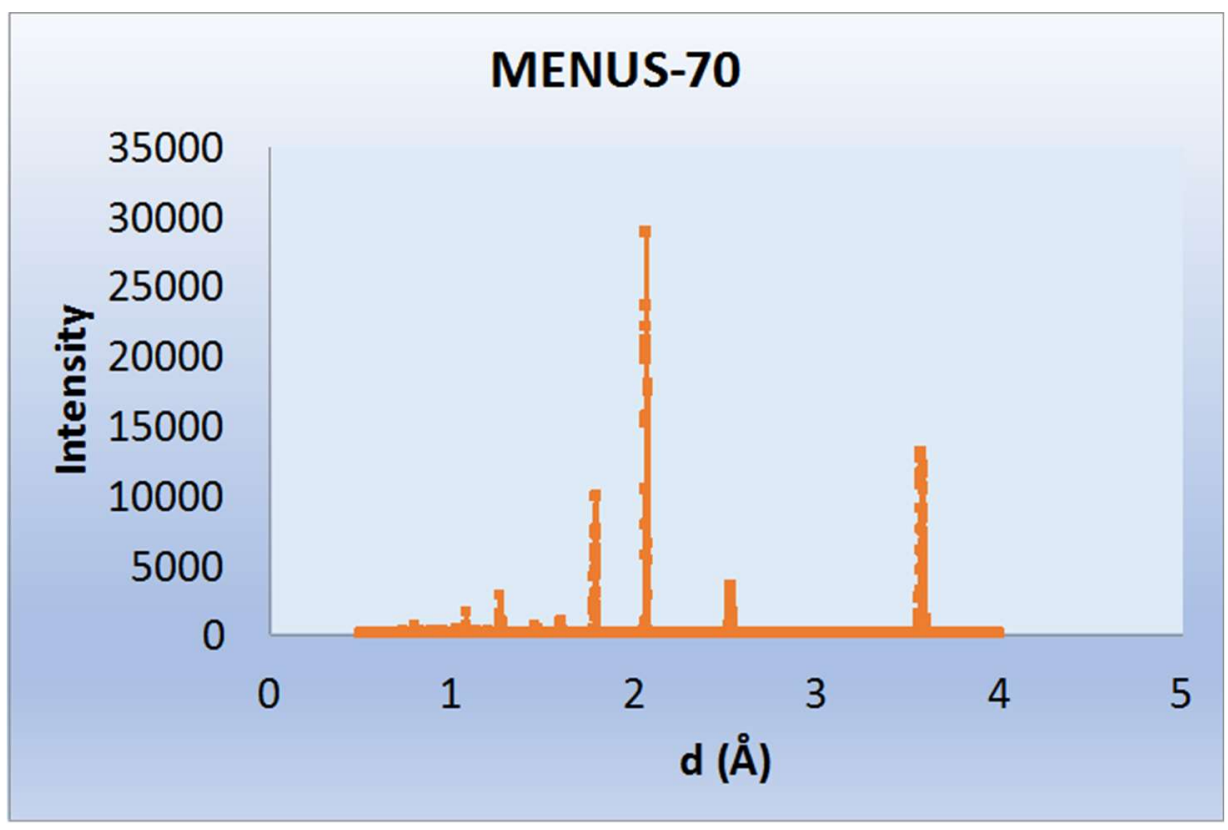

d)

Figure 3.36. MENUS instrument performance. a) flux of MENUS and VULCAN at sample position, and b) Gain in scattering signal of vanadium from simulations and data are compared in ranges where VULCAN can measure at $20 \mathrm{~Hz}$. c) diffraction of $\mathrm{Ni}_{3} \mathrm{Al}$ of by VULCAN. d) diffraction of $\mathrm{Ni}_{3} \mathrm{Al}$ by MENUS 70-degree circular detector. Note the change in vertical scale. (Both data are simulated with same detector type and normalized to same exposure time) 


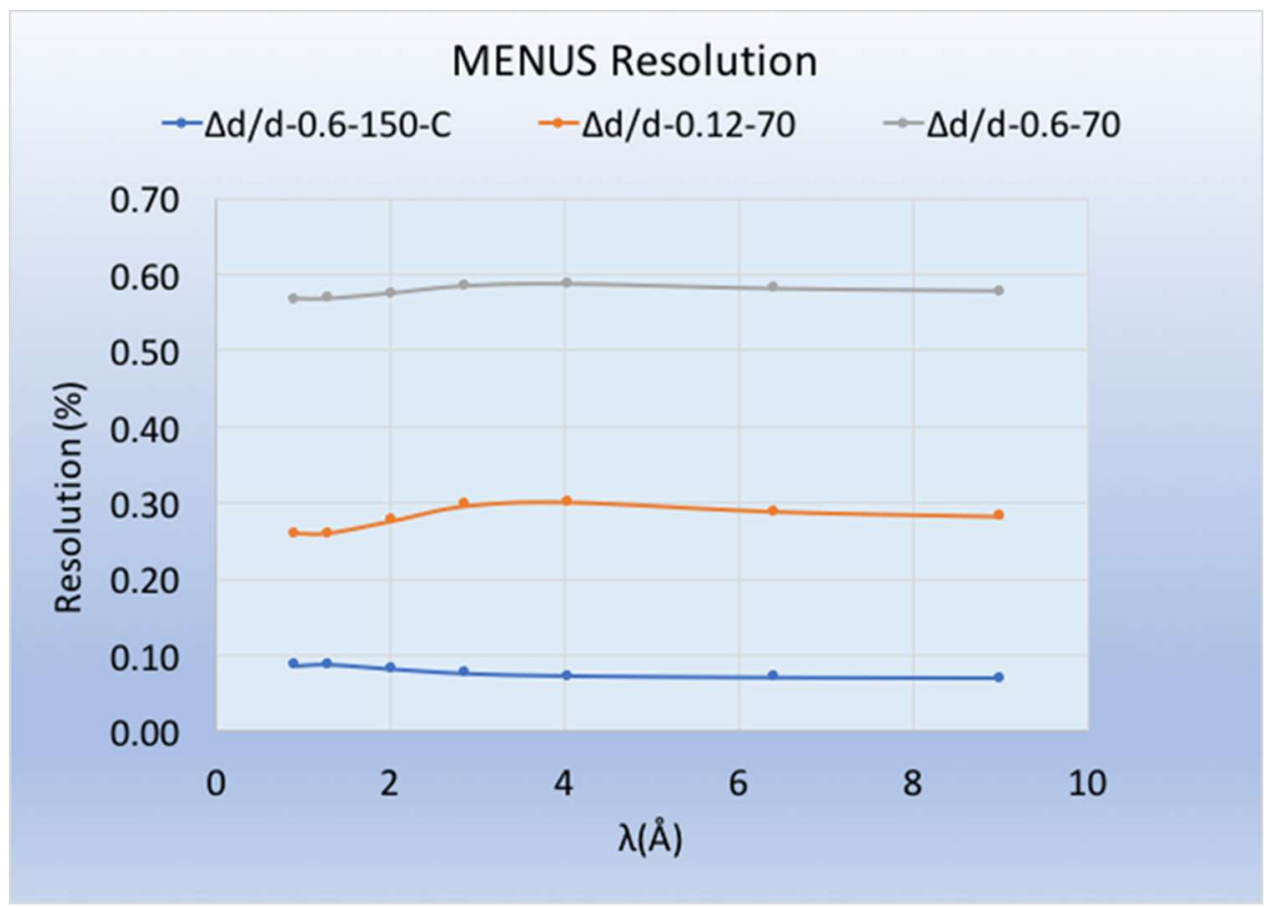

a)

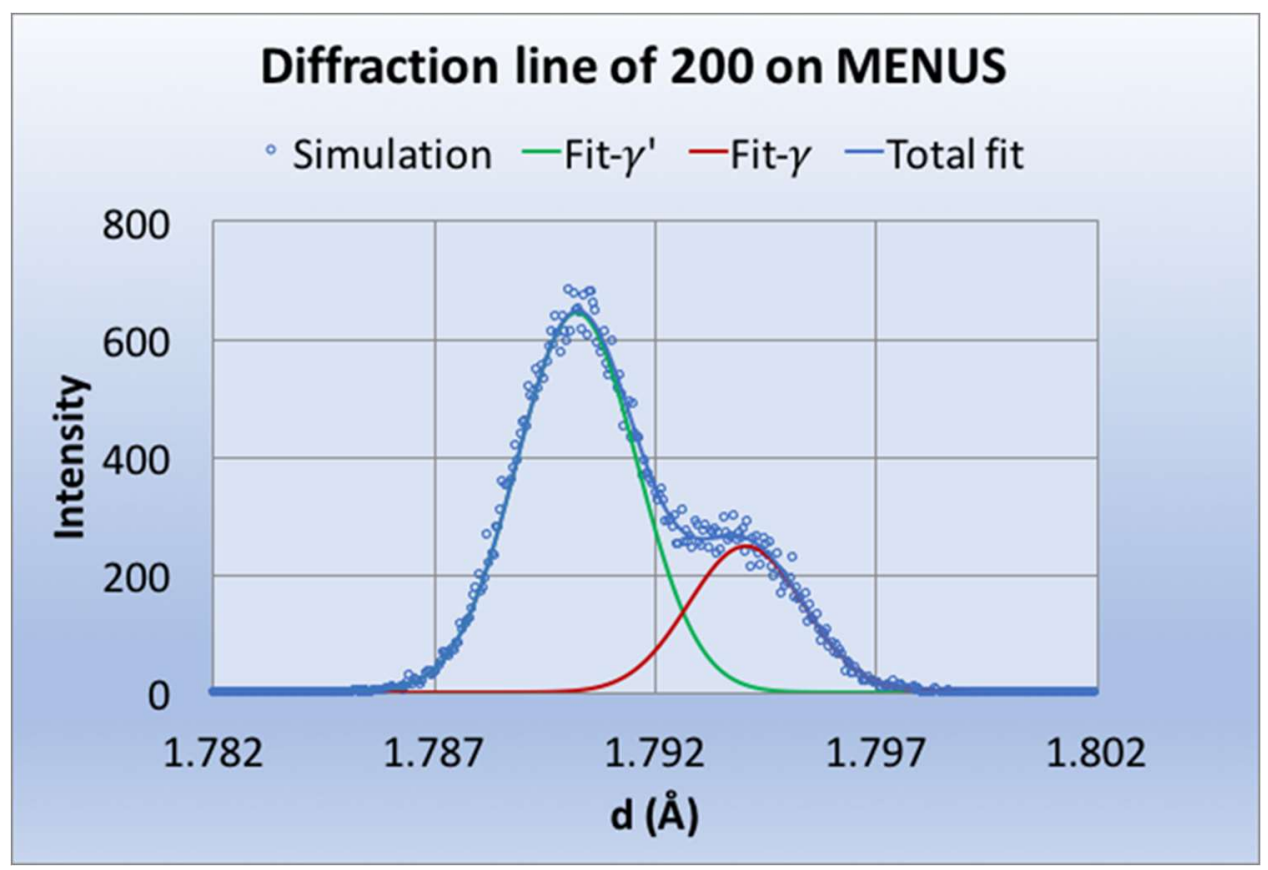

b)

Figure 3.37. MENUS instrument resolution. a) estimated instrument resolution, where, gray line is for the circular detector with beam divergence of 0.6 ; orange line is for the circular detector with beam divergence of 0.12 ; blue line is for the high angle detector of $150^{\circ}$ with beam divergence of 0.6 and correlation chopper. b) separation of (200) peaks in the close misfit $\mathrm{Ni}$ and $\mathrm{Ni}_{3} \mathrm{Al}$ phases simulated at detector at 110 degree with beam divergence of 0.12 . 


\subsection{HiResPD}

\subsubsection{Abstract}

Currently, the highest resolution neutron powder diffractometers in the world are HRPD at ISIS $(\Delta \mathrm{d} / \mathrm{d}=0.04 \%$ at backscattering $)$ and SuperHRPD at JPARC $(\Delta \mathrm{d} / \mathrm{d}=0.035 \%$ at backscattering $)$. The history of high scientific productivity at these instruments and current synchrotron instruments where this resolution has become standard makes a strong case for having an instrument of comparable performance in North America. With the higher intensity available at STS, this instrument will be able to perform high resolution powder diffraction measurement on small samples and will complement the existing suite of powder diffraction instruments at the first target station.

\subsubsection{Scientific Case}

\subsubsection{Hard Condensed Matter:}

Neutron diffraction has greatly enhanced our knowledge of strongly correlated materials and remains one of the principle tools for the determination of magnetic structures. The following two examples highlight the need for higher resolution as we work on more complex systems.

Example 1: Domain formation has a long and rich history in magnetism, and it is the magnetic domains that often enable or hinder the properties of magnetic materials. Recently, magnetic domains with topological properties (e.g., Skyrmions) have risen to the forefront in magnetics/spintronics and topological phases of matter [1,2]. While small ferromagnetic domains can be detected and studied using real-space techniques such as Lorentz transmission electron microscopy, the nature of antiferromagnetic (AFM) domains remains less well understood. A neutron powder diffractometer with exceptionally high resolution at low $Q$ / large $d$ spacing, would lend itself to the study of antiferromagnetic domain behavior.

In a recent series of experiments on the non-centrosymmetric ceramic, $\mathrm{Fe}_{3} \mathrm{PO}_{4} \mathrm{O}_{3}$ (spacegroup $R 3 m$ ), it was shown that the material hosts an unusual helical (or conical) antiferromagnetic structure that organizes into small, needle-like domains ( $\sim 7 \mathrm{~nm}$ in hexagonal $a b$ plane; resolution limited out of plane). The instrument POWGEN at the SNS could detect this finite size broadening of the AFM domains, but only because of the unique materials properties. However, details of AFM domains (such as their average size, growth characteristics) have been reported in very few materials, owing to the challenge in their detection. The primary methods used for their characterization include polarized neutron tomography [3], optical second harmonic generation [4], and photocorrelation spectroscopy [5,6]. The microscopic origin of antiferromagnetic domain formation is poorly understood, as there is no demagnetization energy cost; instead, magnetostriction has been implicated in its origin $[7,8]$. With a neutron powder diffractometer with exquisite resolution, AFM domains may be detected and studied in other magnetic materials in order to be exploited in future technologies. For example, the anisotropic magnetoresistance of antiferromagnetic FeRh has been exploited for a new spintronic device concept [9].

Example 2: Due to their structure and electronic properties, transition metal oxide Ruddlesden-Popper(RP) phases, and in particular nickel-based R-P phases, provide model systems for high temperature cuprate superconducting compounds, and indeed have been predicted to be potential hosts for superconductivity. Although not superconducting, $\mathrm{La}_{4} \mathrm{Ni}_{3} \mathrm{O}_{10}$ (LNO), the $\mathrm{n}=3$ member of the R-P series $\mathrm{La}_{\mathrm{n}+1} \mathrm{Ni}_{\mathrm{n}} \mathrm{O}_{3 \mathrm{n}+1}$ exhibits a weak feature in its electrical resistivity, a metal-to-metal transition at $\sim 140 \mathrm{~K}$, accompanied by a clear heat capacity anomaly. Although theoretical considerations have attributed it to a fermi surface driven charge-density wave (CDW), the origin of this phase transition remains an open question. The potential for CDW, stripes, etc. as 'intertwining' orders with superconductivity motivates this work. 
Understanding the relationship between the structure of LNO above and below the transition is an essential step toward a comprehensive picture of its physics. Remarkably, even the room-temperature structure of LNO is not without controversy, with reports of orthorhombic Fmmm or Cmca, or monoclinic $P 2_{1} / a$, or even lowers symmetry [11-13]. The differences among these structures are quite subtle, involving rigid rotations of $\mathrm{Ni}-\mathrm{O}$ octahedra. The sensitivity of neutrons to oxygen atoms as well as the high resolution at the HiResPD diffractometer will play crucial roles in definitively establishing the correct structure of this nickelate through its metal-to-metal transition.

\subsubsection{Complex Chemical Systems:}

Functional materials of scientific and technological interest, such as zeolitic solids, piezoelectrics, controlled thermal expansion materials, ionic conductors, etc., commonly display a high level of crystallographic complexity (very large unit cell volumes) and/or subtle structural distortions. In many cases, this structural complexity is integral to the useful physical properties of the materials. To properly address such problems using powder diffraction, high resolution data is needed both to resolve subtle splitting in peaks or reveal subtle features in diffraction line shapes and to resolve an adequate number of Bragg peaks at high Q, where overlap and loss of information are significant issues for large unit cells.

Neutrons due to their sensitivity for light elements have been a tool of choice for the characterization of Li-ion battery materials. Despite their great advantage, safety has always been a major concern for lithium-ion systems [14]. This is mainly due to the high operating voltage $(>3 \mathrm{~V})$ and utilization of ignitable liquid or polymer-based organic electrolytes. The failures of $\mathrm{Li}$-ion batteries are often found to occur during the charging process, when the transition metal ions enter higher oxidation states while $\mathrm{Li}^{+}$is extracted from the structure. The delithiated oxide cathode, with large amounts of high valence transition metal ions such as $\mathrm{Co}^{3+}$ or $\mathrm{Ni}^{4+}$, [15] becomes highly activated, such that it may readily be reduced via the release of structural oxygen [16]. This process can result in the oxidation of organic electrolyte, leading to the failure or even explosion of the battery.

One possible mechanism to combat the safety issue in Li-ion batteries is to operate an all solid-state battery, which has a non-flammable solid electrolyte such as $\mathrm{Li}_{7} \mathrm{La}_{3} \mathrm{Zr}_{2} \mathrm{O}_{12}$ (LLZO) garnet. LLZO is a fast ion conductor and is chemically and electrochemically stable against metallic Li. Having demonstrated its viability to enable solid-state batteries, efforts are currently focused on manufacturing and scaling-up synthesis. However, current neutron diffraction analyses carried out at POWGEN $[17,18]$ indicated anomalous crystal lattice effects pointing to inhomogeneous $\mathrm{Li}$ and dopant distribution. It is believed that the synthesis conditions, e.g. time, temperature, and atmosphere, affect phase purity, thus resulting in inconsistent conductivity, stability, and mechanical properties. Detection of the subtle changes in the crystal structure and stoichiometry can only be determined with high-resolution neutron diffraction analysis, which is currently absent in the US neutron sources. These types of experiment can be accomplished by the proposed HiResPD instrument at the STS as shown by Figure 3.38, which was collected on a similar resolution machine at the advanced photon source (APS). However, Li distribution in the multiphase sample could not be obktained from these high-resolution x-ray data due to the insensitivity to $\mathrm{Li}$ and $\mathrm{O}$ in the presence of much heavier $\mathrm{La}$ and $\mathrm{Zr}$. The ability to measure these subtle effects will bolster efforts to mature LLZO solid electrolyte membrane technology to accelerate the development of higher energy density and safer batteries. 


\subsubsection{Ab-initio Structure Solution:}

The collapse of information from 3D to $1 \mathrm{D}$ has always meant that structure solution from powder diffraction data is significantly harder than single crystal. Nevertheless, with improvements in instrumentation, algorithm development and enhanced computing power, great strides have been made in ab-initio structure solution from powder diffraction. While the contrast afforded by difference is $\mathrm{Z}$ often makes $\mathrm{X}$-ray diffraction the method of choice for structure solution, there are however some cases where neutron diffraction is preferred. Indexing and determination of space group is the first step in SDPD for which high resolution data which provides more precise d-spacing information is extremely important.

The sensitivity of neutrons to light elements can play a crucial role in the determination of the correct space group. For example, the tilting of $\mathrm{TiO}_{6}$ in perovskite or orientation of methyl group in small molecules can subtly affect the space group that is difficult to determine from Xray data. Neutron data is used in framework structure to find the light atoms such as hydrogen, $\mathrm{CH}_{4}$ or $\mathrm{CO}_{2}$ inside Clathrate, MOF or Zeolite structures using Fourier cycling. Neutron diffraction data can play a critical role in the structure solution of small molecule and organic inorganic hybrids, heavy metal oxides and other more complex compounds. As the demand for complex structure solution from powder diffraction data grows, the need to combine X-ray and neutron powder diffraction will also increase. Ideally that need can be met by having access to a neutron powder instrument which has the same resolution afforded by synchrotron powder beam lines.

\subsubsection{Conceptual Design}

The High Resolution Powder Diffractometer will complement the existing powder diffraction suite at the first target station. The design at this stage is conceptual and has not been finalized. Therefore, performance estimates are approximate. However, engineering reality, that is, chopper positions, location of the instrument at the chosen moderator (due to the long flight path required to achieve the high resolution) is already part of the design.

\subsubsection{Sample size, beam divergence, wavelength range:}

The HRPD instrument will see the multi-spectral decoupled moderator on cold para-hydrogen side (MSDC) with a $7 \times 7 \mathrm{~cm}^{2}$ cross-section at a sample-moderator distance of about $100 \mathrm{~m}$. A single frame at $15 \mathrm{~Hz}$ will allow a bandwidth of $2.536 \AA$. The bandwidth can be extended up to $7.6 \AA$ by $1 / 3$ frame suppression $(5 \mathrm{~Hz})$. The maximum wavelength range expected to be used spans from 0.5 to $8 \AA$, at $5 \mathrm{~Hz}$ with $1 / 3$ frame suppression, which corresponds to d-spacing range of $0.25-46.5 \AA$ (or $0.1-25 \AA^{-1} \mathrm{Q}$ range) for the full detector coverage spanning from $10^{\circ}$ to $170^{\circ}$ in scattering angle. The instrument resolution will be controlled by tuning of neutron beam divergence at sample position in the $1.2-10$ mrad range. Following is a table of $\mathrm{d}$ spacing that can be accessed in different mode of operation. As compared with the performance of POWGEN instrument at FTS, the improved moderator brightness and the adequate repetition rate will allow a flux gain of 10 for the first frame and over 3 for the next two frames, with 10 mrad beam divergence, which is expected to provide also a significant gain in resolution. 
Table 3.7. HiResPD available operating modes.

\begin{tabular}{ccccccccc}
\hline $\begin{array}{c}\text { Frequency } \\
(H z)\end{array}$ & frame \# $\begin{array}{c}\text { WL } \\
\text { center }(\AA)\end{array}$ & $\begin{array}{c}\text { WL } \\
\min (\AA)\end{array}$ & $\begin{array}{c}W L \\
\max (\AA)\end{array}$ & $\operatorname{dmin}(\AA)$ & $\operatorname{dmax}(\AA)$ & $\begin{array}{c}\text { Qmin } \\
\left(\AA^{-1}\right)\end{array}$ & $\begin{array}{c}\text { Qmax } \\
\left(\AA^{-1}\right)\end{array}$ \\
\hline 5 & 1 & $\mathbf{3 . 8 0 4}$ & $\mathbf{0 . 5}$ & $\mathbf{8 . 1 0 8}$ & $\mathbf{0 . 2 5 1 0}$ & $\mathbf{4 6 . 5 3 8 0}$ & $\mathbf{0 . 1 3 5 0}$ & $\mathbf{2 5 . 0 3 5 4}$ \\
7.5 & 1 & 2.536 & $\mathbf{0 . 5}$ & $\mathbf{5 . 5 7 2}$ & $\mathbf{0 . 2 5 1 0}$ & $\mathbf{3 1 . 9 8 1 9}$ & $\mathbf{0 . 1 9 6 5}$ & $\mathbf{2 5 . 0 3 5 4}$ \\
15 & 1 & 1.268 & $\mathbf{0 . 5}$ & $\mathbf{3 . 0 3 6}$ & $\mathbf{0 . 2 5 1 0}$ & $\mathbf{1 7 . 4 2 5 9}$ & $\mathbf{0 . 3 6 0 6}$ & $\mathbf{2 5 . 0 3 5 4}$ \\
15 & $\mathbf{2}$ & 4.304 & $\mathbf{3 . 0 3 6}$ & $\mathbf{5 . 5 7 2}$ & $\mathbf{1 . 5 2 3 9}$ & $\mathbf{3 1 . 9 8 1 9}$ & $\mathbf{0 . 1 9 6 5}$ & $\mathbf{4 . 1 2 3 1}$ \\
15 & 3 & $\mathbf{6 . 8 4}$ & $\mathbf{5 . 5 7 2}$ & $\mathbf{8 . 1 0 8}$ & $\mathbf{2 . 7 9 6 8}$ & $\mathbf{4 6 . 5 3 8 0}$ & $\mathbf{0 . 1 3 5 0}$ & $\mathbf{2 . 2 4 6 5}$ \\
\hline
\end{tabular}

\subsubsection{HRPD optics design:}

Figure 3.39 shows the HRPD optics design. A parabolic guide, $11.2 \mathrm{~m}$ long, starts at $8 \mathrm{~m}$ from the moderator and has its focus located on the moderator surface in both horizontal and vertical plane. Its role is to transform the divergent beam coming from the moderator to a wider and better collimated beam. Also, the entrance aperture of the guide $(63.8 \mathrm{~mm}$ square $)$ will restrict the direct field of view from the sample position to the size of the moderator (Figure 3.39b). The exit width and height of the guide reaches $98.8 \mathrm{~mm}$. The next guide has an elliptic profile, with the two focal points located at the entrance in the first piece of guide and at the sample position. It is a long guide (68.66 m in length), which starts $19.88 \mathrm{~m}$ from the moderator. The entrance and exit size is almost the same, $97.94 \mathrm{~mm}$ and $96.42 \mathrm{~mm}$, respectively, and the maximum lateral extension reaches $146 \mathrm{~mm}$. The elliptical shape of the mirror surface ensure the neutron travel of more divergent paths from moderator to the sample with a single reflection event in both horizontal and vertical plane, and maintaining the reflection angle at a minimal value (Figure 3.39c). For the first two components of the guide, a supermirror deposition of $m=3$ would be good enough to reflect all neutrons with wavelengths larger than $0.5 \AA$. The last piece of guide was introduced to further enhance the beam divergence. It is a short parabolic guide $(2.01 \mathrm{~m})$ focusing at sample position. The entrance $32.1 \mathrm{~mm}$ square is located at $95.63 \mathrm{~m}$ from the moderator $(4.37 \mathrm{~m}$ from sample position). The exit $23.6 \mathrm{~mm}$ square, located at $2.36 \mathrm{~m}$ from sample position, defines the maximum divergence angle to about $10 \mathrm{mrad}$. The parabolic shape of the reflecting walls will allow collecting to the sample position the neutrons coming directly from the moderator (Figure 3.39d), as well as some neutrons reflected once inside one of the other pieces of guide Figure 3.39e and 3.39f). Two adjustable square apertures located before and after the last piece of guide will allow tuning the beam divergence. This piece of guide will require high quality $(\mathrm{m}=5)$ supermirror. The acceptance diagram for the neutron beam at the sample position is presented in Figure $3.39(\mathrm{~g})$. It can be seen that the guide system provide a continuous angular coverage of $10 \mathrm{mrad}$ for spatial coordinates within $7.5 \mathrm{~mm}$ range across sample, which corresponds to about $0.5 \mathrm{~mm}^{2}$ sampling area. 


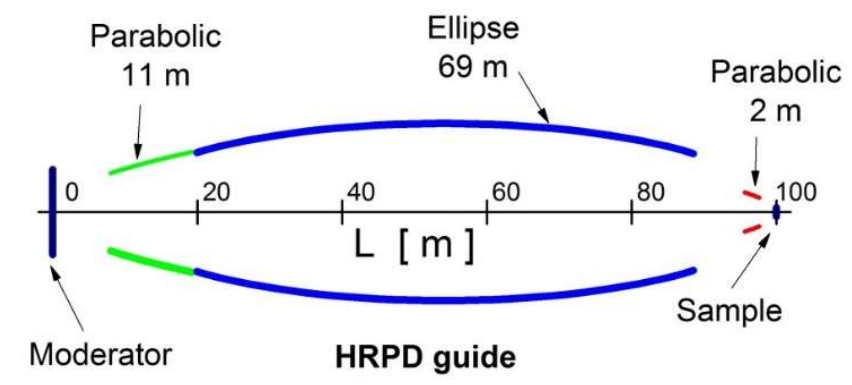

(a)

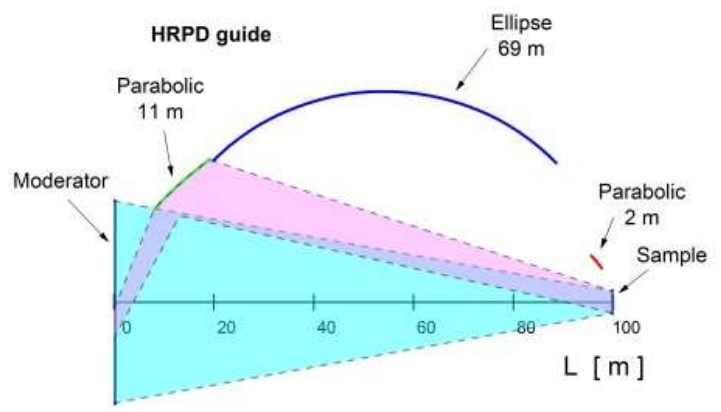

(b)

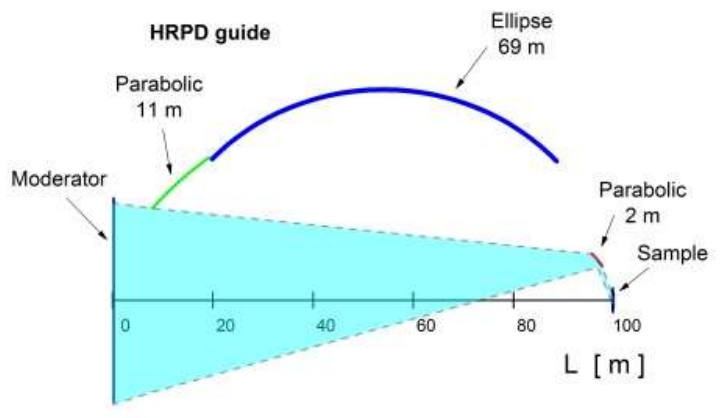

(d)

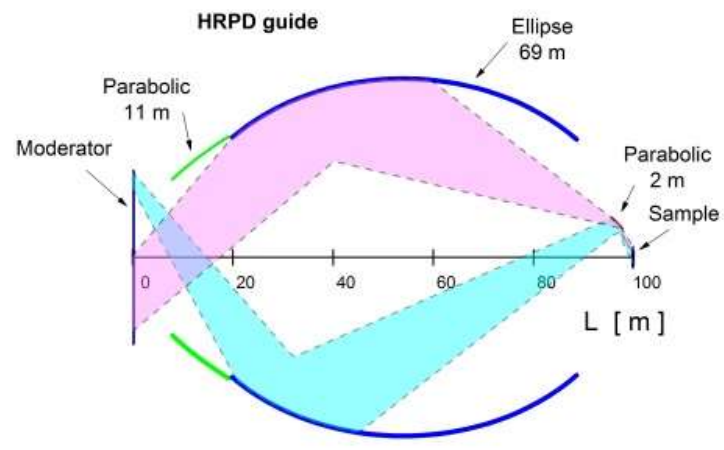

(f)

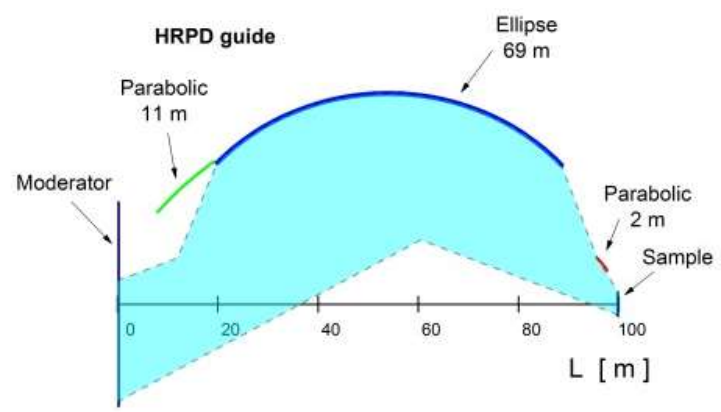

(c)

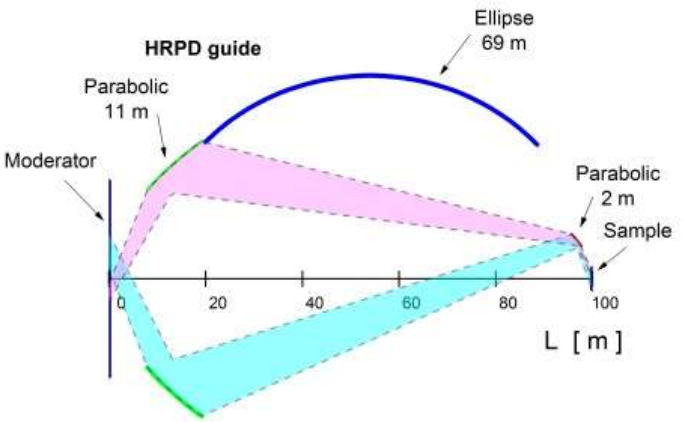

(e)

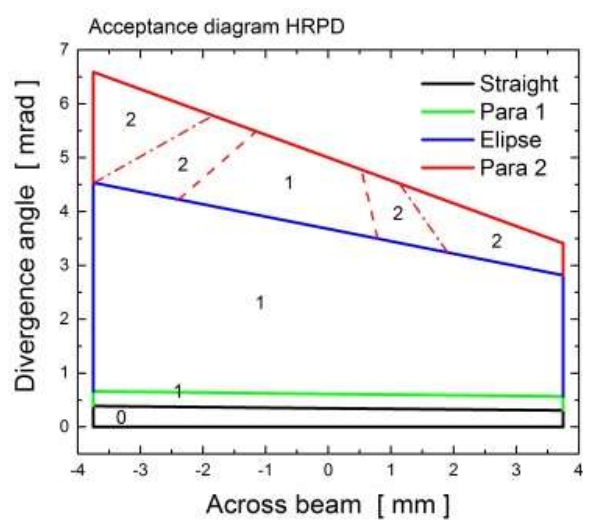

(g)

Figure 3.39. The HRPD guide system. (a) schematic view; ray tracing: (b) first parabolic mirror, (c) elliptic mirror, (d) second parabolic mirror, (e) double reflection (parabolic-parabolic), (f) double reflection (elliptic-parabolic); and (g) space-angle acceptance diagram of whole system. 


\subsubsection{Choppers and timing:}

The frame definition choppers functionality is determined by the total neutron flight path including moderator-sample $(100 \mathrm{~m})$ and maximum sampledetector distance $(4 \mathrm{~m})$. The chopper timing graph is presented in Figure 3.40. The first chopper (double disk) is located at $7.76 \mathrm{~m}$ from moderator and has the disk opening of about $33.5^{\circ}$. The double disk design provides a reduced opening time and allows for changing the wavelength bandwidth, which is a feature required by some special applications. Beside the working bandwidth, the first chopper permits slow neutrons (34 $37 \AA$ ) to reach the detector location; a second chopper, with a disk opening of $78^{\circ}$, was placed at $19.5 \mathrm{~m}$ from the moderator to absorb these unwanted neutrons.

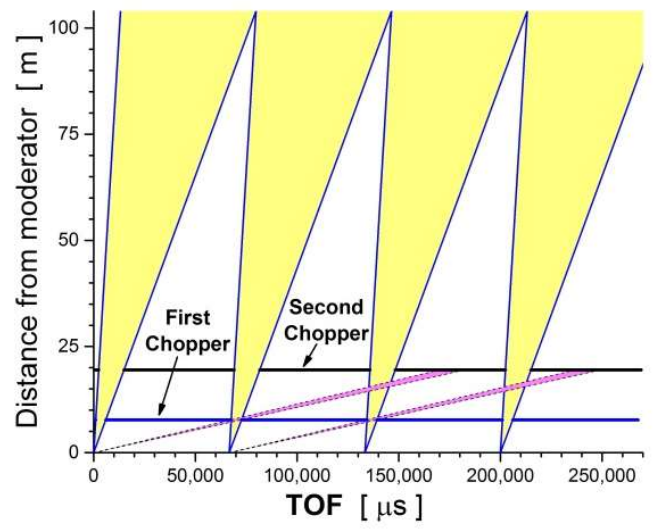

Figure 3.40. The HRPD timing diagram.

\subsubsection{Detector layout and type}

The detectors layout follows the same scheme as POWGEN $\left(4.75 \mathrm{~m}\right.$ at $10^{\circ}$ and $2 \mathrm{~m}$ at $\left.170^{\circ}\right)$; thus, the detector locus was considered to follow the same expression for an equiangular spiral as POWGEN:

$$
r=\mathrm{A} \exp (\mathrm{B} \theta) ; A=4.7 m, B=-0.287 \mathrm{rad}^{-1}
$$

Each detector bank will include a double layered array of $8 \mathrm{~mm} \mathrm{He} 3$ tube detectors, which provide a spatial resolution of about $6 \mathrm{~mm}$ along their axes. As the beam divergences in the horizontal and vertical plane are equivalent, the out of plane detector coverage is not resolution limited. The sample environment arrangement will constrain the useful detector coverage extension.

The instrument will take full advantage of the large bandwidth offered by running in frame suppression mode when the experiment requires accessing a large d-spacing range. However, it can also operate in $15 \mathrm{~Hz}$ mode and, depending on the resolution needs, different frames can be picked.

\subsubsection{Engineering model}

The conceptual design of HRPD is shown in Figure 3.41. The longest flight path (L1) that is possible given the surrounding geography is $100 \mathrm{~m}$. 


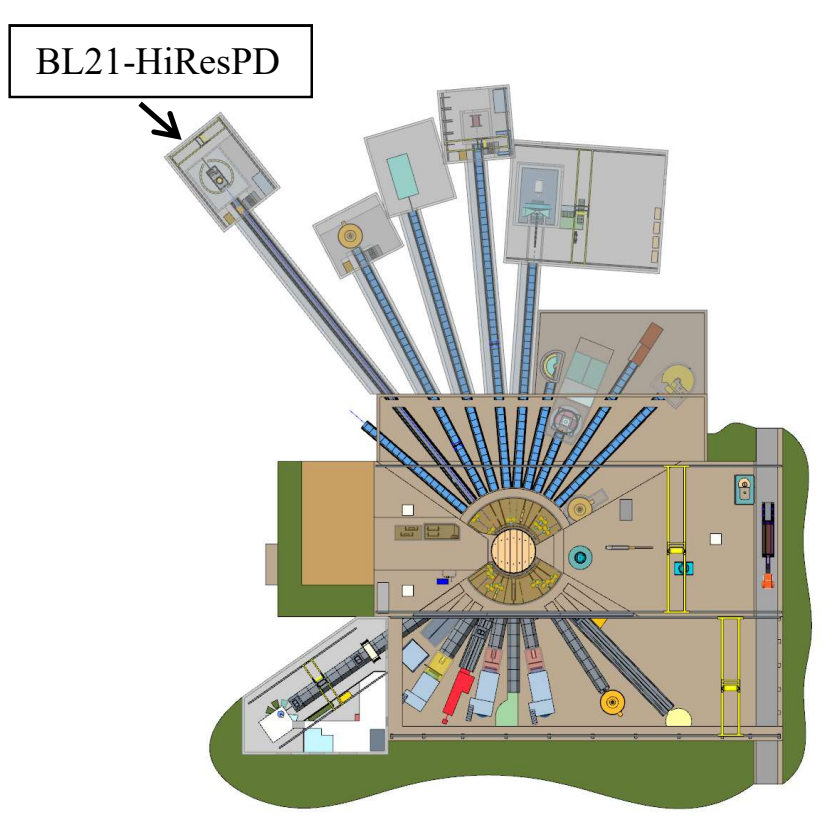

Figure 3.41. The engineering model of HRPD.

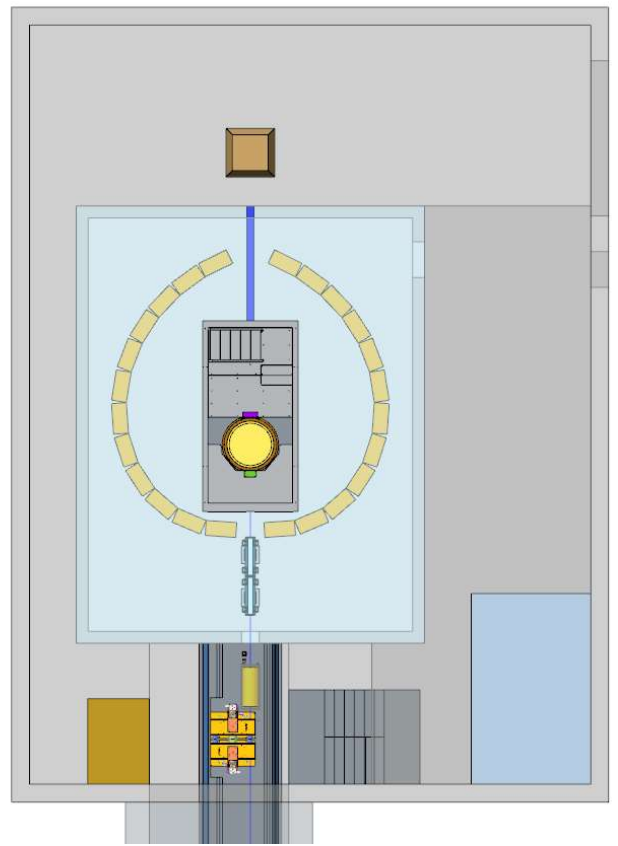

(a) beamline layout and b) detector coverage

\subsubsection{HRPD performance estimation in flux and resolution}

The expected flux at the sample position was calculated starting from simulated modarator brightensss, taking into acount the acceptance diagram and the average number of reclections inside the guide system. The estimated values correspond to the $15 \mathrm{~Hz}$ mode of operation, which correspons to $700 \mathrm{~kW}$ on STS (Figure 3.42). The POWGEN data are the results of measurements. The resolution estimations consider the pulse widths resulting from moderator simulations and include beam divergence contribution, as well as the sample size $(\sim 5 \mathrm{~mm})$ and the detector spatial resolution. As the resolution dependence of wavelength was week, the data represented in Figure 3.42 are average values for fixed scattering angle. The minimum value of resolution is reched at $170^{\circ}$ scattering angle and amounts to $0.038 \%, 0.032 \%$ and $0.024 \%$ for different divergence levels: $10 \mathrm{mrad}, 7.35 \mathrm{mrad}$ and $1.22 \mathrm{mrad}$, respectively.
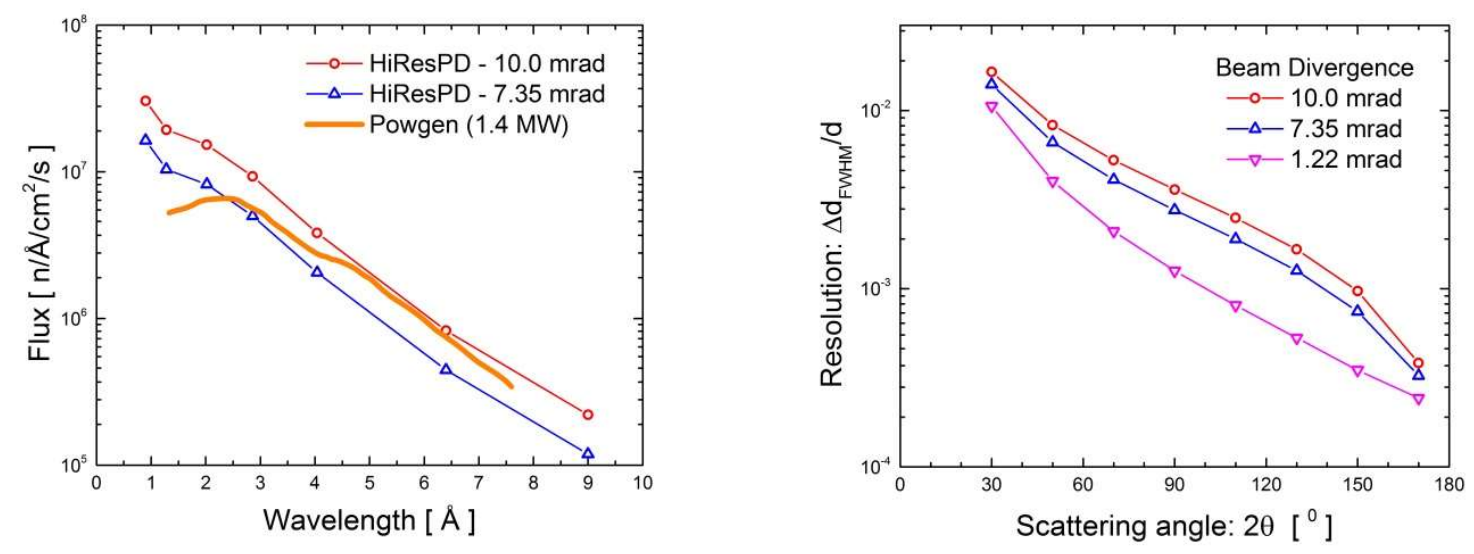

Figure 3.42. HRPD performance. (a) Neutron flux of HRPD at sample position and (b) Resolution of HRPD as function of scattering angles. 


\subsubsection{References}

[1] Ma E.Y., Cui Y.T., Ueda K., Tang S., Chen K., Tamura N., Wu P.M., Fujioka J., Tokura Y. and Shen Z.X., Science, 350(6260), 538 (2015).

[2] Goltsev A.V., Pisarev R.V., Lottermoser T. and Fiebig M., Phys. Rev. Lett, 90(17), 177204 (2003).

[3] Schlenker M. and Baruchel J., J. Appl. Phys. 49, 1996 (1978).

[4] Van Aken B. B., Rivera J.P., Schmid H. and Fiebig M., Phys. Rev. Lett. 101, 157202 (2008).

[5] Shpyrko O., Isaacs E., Logan J., Feng Y., Aeppli G., Jaramillo R., Kim H., Rosenbaum T., Zschack P., Sprung M. et al., Nature (London) 447, 68 (2007).

[6] Chen S.W., Guo H., Seu K. A., Dumesnil K., Roy S., and Sinha S. K., Phys. Rev. Lett. 110, 217201 (2013).

[7] Minakov A., Shvets I., and V. Veselago, J. Magn. Mater. 88, 121 (1990).

[8] Gomonay H. and Loktev V. M., J. Phys.: Condens. Matter 14, 3959 (2002).

[9] Marti X., Fina I., Frontera C., Liu J., Wadley P., He Q., Paull R.J., Clarkson J.D., Kudrnovský J., Turek I. and Kuneš J., Nature Materials. 13(4), 367 (2014).

[10] Ross K.A., Bordelon M.M., Terho G. and Neilson J.R., Phys. Rev. B, 92,134419 (2015).

[11] Zhang Z \& Greenblatt M (1995) Synthesis, structure, and properties of $\mathrm{Ln}_{4} \mathrm{Ni}_{3} \mathrm{O}_{10-\mathrm{d}}$ ( $\mathrm{Ln}=\mathrm{La}, \mathrm{Pr}$, and Nd). J. Solid State Chem. 117(2):236-246.

[12] Ling CD, Argyriou DN, Wu G, \& Neumeier JJ (2000) Neutron diffraction study of $\mathrm{La}_{3} \mathrm{Ni}_{2} \mathrm{O}_{7}$ : structural relationships among $n=1,2$, and 3 phases $\mathrm{La}_{n+1} \mathrm{Ni}_{n} \mathrm{O}_{3 n+1}$. J. Solid State Chem. 152(2):517525 .

[13] Olafsen A, Fjellvåg H, \& Hauback BC (2000) Crystal Structure and Properties of $\mathrm{Nd}_{4} \mathrm{Co}_{3} \mathrm{O}_{10+\delta}$ and

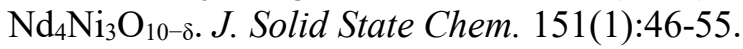

[14] Dahn J., Fuller E., Obrovac M. and Von Sacken U., Solid State Ionics 69 (1994): 265-270

[15] Jiang J. and Dahn J., Electrochem. Commun. 6 (2004): 39-43.

[16] Mizushima K., Jones P., Wiseman, P. and Goodenough J. B., Mater. Res. Bull. 15 (1980): 783-789.

[17] Thompson T., Wolfenstine J., Allen J. L., Johannes M., Huq A., David I. N., and Sakamoto J., J. of Mat. Chem. A, 2 (2014): 13431-13436.

[18] Thompson T., Sharafi A., Johannes M. D., Huq A., Allen J. L., Wolfenstine J., and Sakamoto J., Advanced Energy Materials, 5 (2015): 1500096. 


\section{MODERATOR PERFORMANCE}

\subsection{INTRODUCTION}

STS was designed and optimized to provide high-intensity long-wavelength neutron beams at low source frequency to enable broad bandwidth measurements [1]. Even at reduced power of $\sim 0.5 \mathrm{MW} @ 10 \mathrm{~Hz}$, which was the TDR concept, compared to FTS designed for 2MW@60Hz, moderator materials other than ambient temperature water and liquid hydrogen are at present out of question.

As nature precludes a change of neutron phase-space density by conservative forces and by the use of optics components, the only way to achieve increased flux at the sample position for given neutron beam divergence is by providing higher brightness (number of neutrons emitted per unit area and per unit solid angle from moderator port) sources. Four design measures were applied towards this goal for designing the STS target station:

- Tungsten, a high- $Z$ material providing a high material density of $19.3 \mathrm{~g} / \mathrm{cm}^{3}$, was chosen as target material and arranged into a rotating target to minimize the cooling medium fraction in the target volume. The $1.3 \mathrm{GeV}$ proton beam with about $30 \mathrm{~cm}^{2}$ footprint will produce a compact neutron production zone that perfectly feeds the moderators grouped around it.

- Coupled para-hydrogen moderators were positioned in the wing position above and below the target to complement FTS, which has decoupled poisoned moderators in the prime position as its emphasis was on producing narrow neutron pulses from the moderators. The cold moderators are supplied with liquid para-hydrogen at $20 \mathrm{~K}$ temperature and will require ortho-to-para catalyzed hydrogen loop. Their configuration together with ambient temperature water pre-moderator was optimized to provide the desired performance metrics.

- The moderator view port was down-sized as only a limited size of moderator can contribute to illuminate small samples. Especially the reduction of moderator height pulls the moderator volume closer towards the target, provides better coupling to the reflector and enhances the overall feed-in of the moderators.

- Doubling of the accelerator power from 1.4 MW to $2.8 \mathrm{MW}$ at the same repetition rate of $60 \mathrm{~Hz}$ will double the proton energy per pulse and hence double the neutron yield per pulse.

Sizable gains of moderator performance were achieved over comparable FTS moderators as outlined in the following sections.

\subsection{DETAILS OF SIMULATIONS}

Initially, simulations were conducted to optimize moderator-target-reflector arrangements towards peak pulse cold brightness $(\mathrm{E}<5 \mathrm{meV})$ for various view port sizes ranging from $2 \times 2$ to $10 \times 10 \mathrm{~cm}^{2}$ for cylindrical coupled para-hydrogen moderators viewed at three ports [2]. Likewise, a decoupled box-type hydrogen moderator was optimized to provide the pulse width comparable to those provided by the FTS decoupled hydrogen moderator. An instrument optics study was conducted concluding that the brightness gains of the moderators with smaller viewed area translate to superior fluxes particularly when instruments study smaller sample sizes [3].

In parallel, a suite of 19 STS candidate instruments was developed [1]. The source requirements of these instruments lead to a selection of three moderators to be grouped around the target: 
- a coupled cylindrical para-hydrogen moderator with two view ports of $3 \times 3 \mathrm{~cm}^{2}$ and one of $3 \times 6 \mathrm{~cm}^{2}$ provides 10 beams,

- a box-type coupled para-hydrogen moderator with $5 \times 5 \mathrm{~cm}^{2}$ view port serves 3-4 instruments,

- a decoupled thermal/cold moderator with one side of ambient temperature water and the other side para-hydrogen both with view ports of $7 \times 7 \mathrm{~cm}^{2}$ illuminating 6 beams.

These three moderators were successively optimized and optimally placed around a stationary target as described by Remec [4]. The decoupled water moderator side was optimized to provide the pulse width of the thick high-intensity side of the FTS water moderator. To probe the impact of optimizing to highest achievable time-averaged brightness, we also optimized the cylindrical moderator with 3 -cm-high view ports for high time-integrated brightness. The radial dimension of the moderator grew by a factor of 2.5.

As the stationary target was deemed to present challenges in the loss-of-coolant accident scenario, it was later replaced by a rotating tungsten target of the same height as the stationary target [5]. In addition to solving all cooling problems, this change gave slight advantages in terms of neutron yield as water cooling channels between the tungsten plates in the stationary target concept could be eliminated.

The moderator suite resulting from the first round of optimizations is idealistically shaped with fixed height, flat moderator vessel walls and fully converted para-hydrogen. It is to be considered as guidance for the engineering design. In their exact shape, the moderators would be difficult to manufacture considering stress constraints; compromises will have to be made and some degradation of the performance presented here may result. Ultimately, the moderator suite will be further optimized based on instrument specific metrics as those become available and on more realistic geometry as engineering constraints are included. A second round of moderator optimization is planned for FY2017.

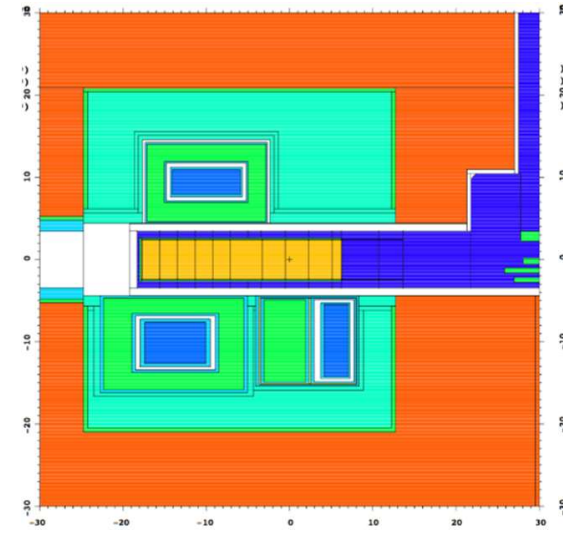

a) vertical cut,

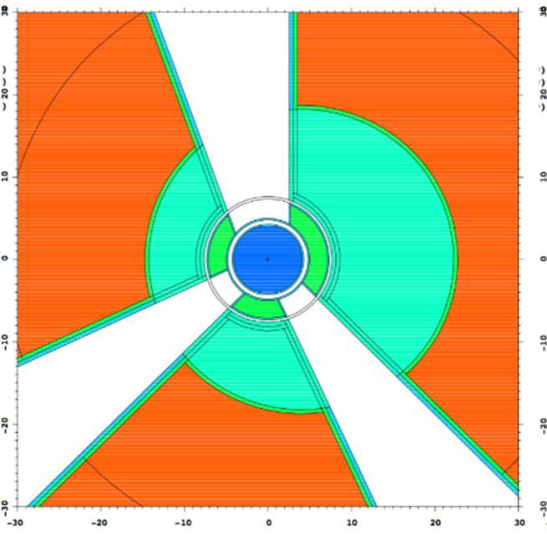

b) horizontal cut top moderator,

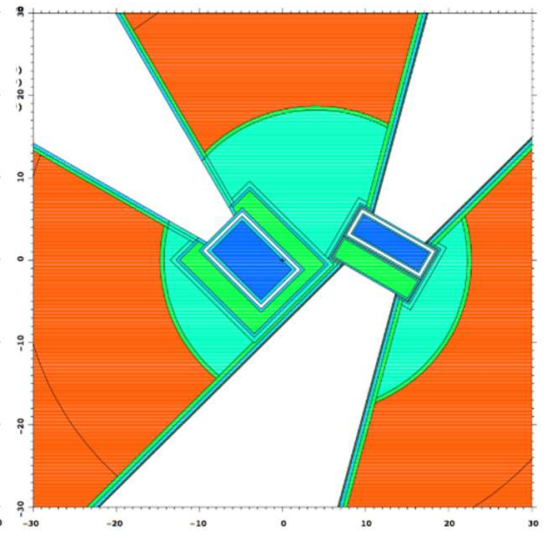

c) horizontal cut bottom moderator

Figure 4.1. MCNPX model of optimized STS target/moderator/reflector arrangement. Steel target housingdark blue; tungsten insert-orange; hydrogen moderator-blue; light water - green; beryllium reflector -turquoise; aluminum structure - light blue; steel shielding - red.

\subsection{RESULTS}

Figures 4.2 and 4.3 show time-integrated brightness, peak brightness, and full-width-at-half-maximum pulse widths for the coupled and decoupled moderators, respectively, on the left side presented versus energy and on the right presented versus wavelength. 

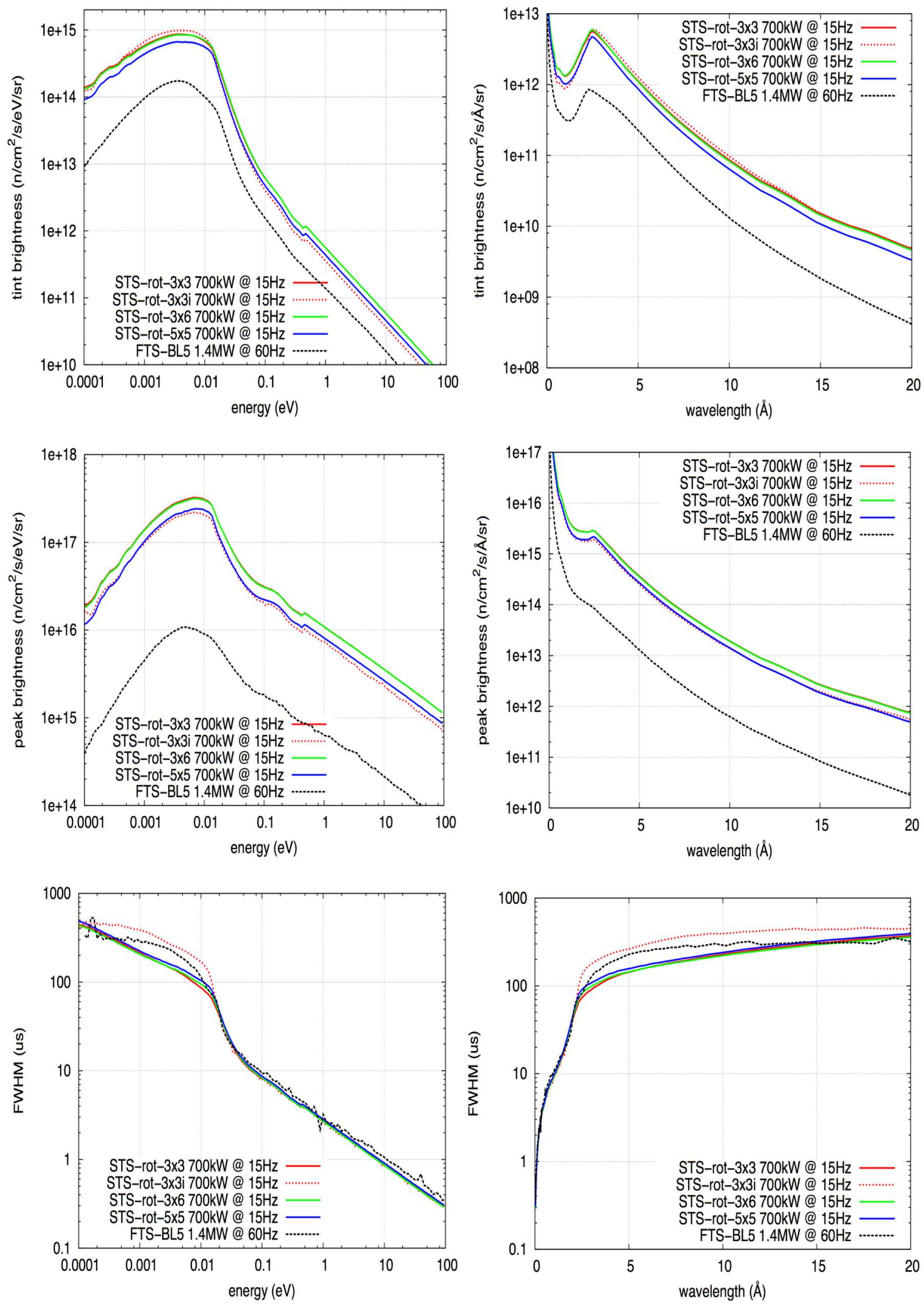

Figure 4.2. Comparison of cold coupled moderator performance metrics, of time-averaged and peak brightness and FWHM pulse width, of STS and FTS moderators vs energy(left) and vs wavelength (right). 

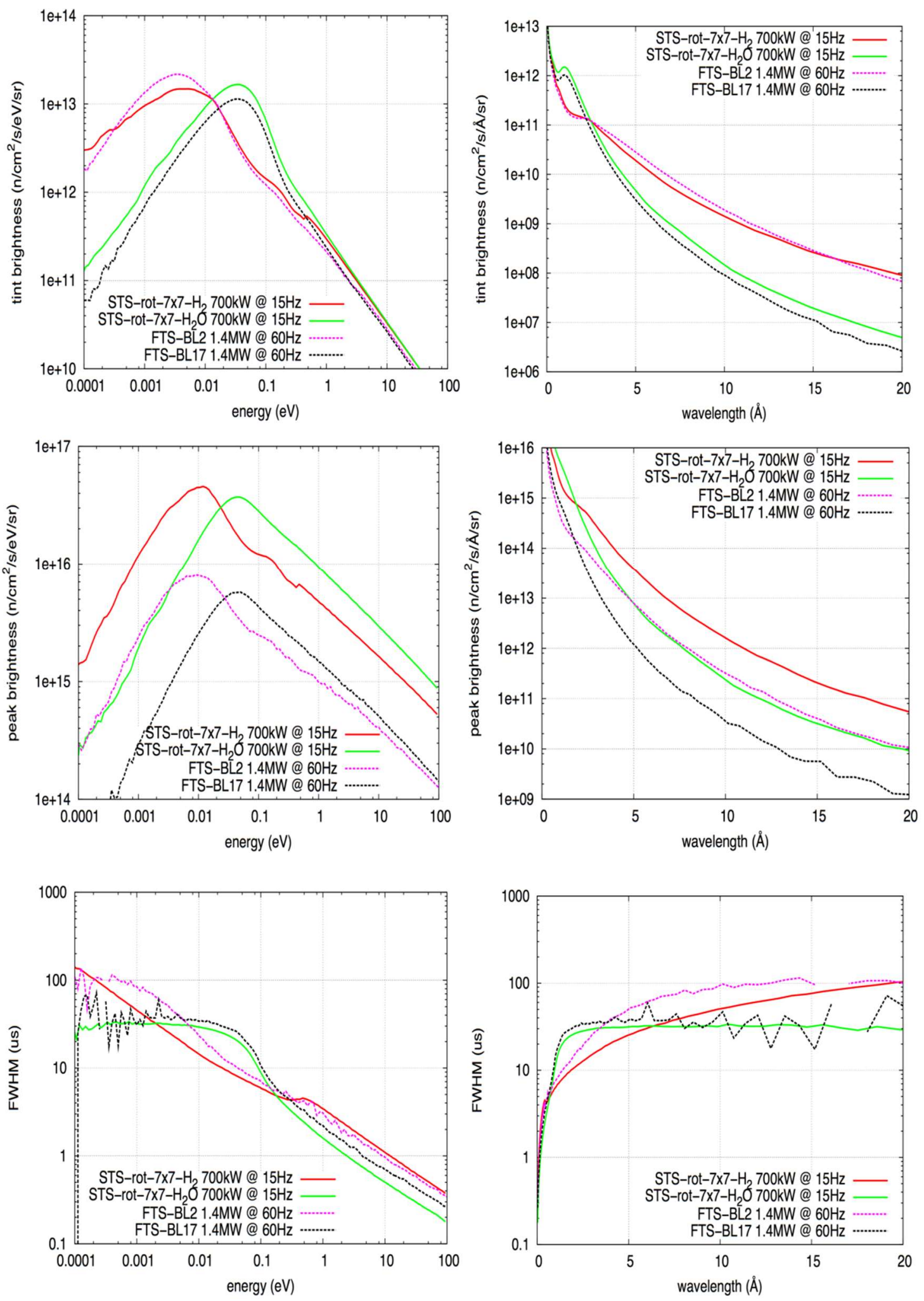

Figure 4.3. Comparison of cold decoupled hydrogen and ambient temperature water moderator performance metrics, of time-averaged and peak brightness and FWHM pulse width, of STS and FTS moderators vs energy(left) and vs wavelength (right). 
As baseline for comparison, FTS in the present design and operating conditions was chosen $(1.0 \mathrm{GeV}, 1.4$ $\mathrm{MW}, 60 \mathrm{~Hz}$ ) [6]. After implementation of the power upgrade, FTS may operate with a beam of $1.3 \mathrm{GeV}$ proton energy, $2 \mathrm{MW}$ power, and $60 \mathrm{~Hz}$. Improvements in neutron efficiency of $2 \%$ and $7 \%$, for decoupled and coupled moderator, respectively, are expected by the change in proton energy from 1.0 to $1.3 \mathrm{GeV}$ and will boost the brightness gains anticipated from the power increase. In addition, gains due to facility improvements are anticipated: 5\% from replacing the Inconel proton beam window with an aluminum window; and $22 \%$ and $10 \%$ from converting the inner reflector plug cooling from light water to heavy water for decoupled and coupled moderators, respectively. These improvements, including the power-increase provided by PPU are expected to produce factors of up to 1.7-1.8 higher intensities for FTS by the time STS begins operation.

Table 4.1. Gains over the present FTS at 1.4 MW and STS moderators with results as high as 6 and 30 for the smallest sized coupled moderator in time-integrated brightness, and peak brightness, respectively

\begin{tabular}{lccc}
\hline \multirow{2}{*}{ Moderator } & $\begin{array}{c}\text { Optimization } \\
\text { brightness metric }\end{array}$ & $\begin{array}{c}\text { Time-integrated } \\
\text { brightness }\end{array}$ & Peak brightness \\
\cline { 3 - 4 } & peak & 6 & 30 \\
STS coupled para- $\mathrm{H}_{2} 3 \times 3 \mathrm{~cm}^{2}$ & integral & 7 & 20 \\
STS coupled para- $\mathrm{H}_{2} 3 \times 3 \mathrm{~cm}^{2}$ & peak & 6 & 30 \\
STS coupled para- $\mathrm{H}_{2} 3 \times 6 \mathrm{~cm}^{2}$ & peak & 5 & 22 \\
STS coupled para- $\mathrm{H}_{2} 5 \times 5 \mathrm{~cm}^{2}$ & FTS-fwhm & 1.5 & 6 \\
STS decoupled para- $\mathrm{H}_{2} 7 \times 7 \mathrm{~cm}^{2}$ & FTS-fwhm & 1.2 & 5 \\
STS decoupled $\mathrm{H}_{2} \mathrm{O} 7 \times 7 \mathrm{~cm}^{2}$ & &
\end{tabular}

All STS coupled moderators clearly benefit from the STS design choices by giving peak brightness gains of as much as a factor of 30 relative to the comparable FTS moderator (this is partially attributable to the unfavorable positioning of the FTS coupled moderators relative to peak neutron production and to their ortho-hydrogen content). The STS decoupled moderator benefits from the more compact STS neutron production zone and smaller moderator size to compare favorably with their FTS counterparts even though it is located downstream from the peak STS neutron production region.

For the coupled cylindrical para-hydrogen moderator, we also report data for the version optimized for peak brightness (STS-rot-3x3) and for time-integrated brightness (STS-rot-3x3i). The time-integratedbrightness-optimized version delivers $17 \%$ higher time-integrated brightness at a $27 \%$ loss in peak brightness with accompanying increased neutron pulse widths compared to the peak brightness-optimized version.

\subsection{REFERENCES}

[1] J. D. Galambos et al., Technical Design Report Second Target Station, ORNL/TM-2015/24, Oak Ridge National Laboratory, Oak Ridge, Tennessee, USA (January 2015).

[2] F. X. Gallmeier et al., Conceptual moderator studies for the Spallation Neutron Source short-pulse second target station, Rev Sci Instrum. 87(6), 063304 (2016), doi: 10.1063/1.4953612.

[3] Zhao et al., Optimizing moderator dimensions for neutron scattering at the spallation neutron source, Review of Scientific Instruments 84, 125104 (2013); doi: 10.1063/1.4841875

[4] I. Remec at al., "Neutronic Analyses for the SNS Second Target Station," Proceedings of the ANS MC2015 - Joint International Conference on Mathematics and Computation (M\&C), 
Supercomputing in Nuclear Applications (SNA) and the Monte Carlo (MC) Method, Nashville, Tennessee, April 19-23, 2015, Vol. 4, pp. 2713-2726 (2015).

[5] I. Remec et al., Second Target Station Moderator Performance with a Rotating Target, in Proceedings: 12th International Topical Meeting on Nuclear Applications of Accelerators (AccApp 2015), Washington, DC, November 10-13, 2015, ISBN: 978-0-89448-724-8.

[6] W. Lu, Moderator Performance of the SNS as-built Configuration, SNS-106100200-TR0199-R00, January 2013. 


\section{TARGET AND INSTRUMENT ENGINEERING}

\subsection{OVERVIEW}

\subsubsection{Physics Requirements}

The neutronics and instrument requirements for the Second Target Station (STS) preceded the initial target station design by over a year [1][2]. Chief among these requirements was the early determination that a short pulse, long wave length facility fit into the three facility, neutron science mission of ORNL and the DOE office of Basic Energy Sciences. Because the STS will share the SNS accelerator the proton beam operational parameters were optimized for pulse sharing of an up-graded machine operating at 1.3 $\mathrm{GeV}$. It was also established that the STS should accommodate up to 22 instrument beam lines to maximize neutron utilization.

The physics studies assumed the target station would have the same basic configuration used or proposed for most accelerator-based facilities (Figure 5.1) built or proposed in the last two decades. In this arrangement, the target is located inside a heavily shielded monolith with the proton beam entering horizontally. Moderators, surrounded by reflectors, are mounted above and below the target assembly and the neutron beam guides are arrayed on either side of the monolith. This has become the preferred arrangement since it simplifies proton beam routing, is inherently safe in the event of beam excursions and is compatible with the optimum moderator-beam line configurations.

Source optimizations (described in Section 4.1 ) established the need for a compact proton beam (30 $\mathrm{cm}^{2}$ ); small, cryogenic hydrogen moderators and beam guides ranging in size from $7 \mathrm{~cm} \mathrm{x} 7 \mathrm{~cm}$ to $3 \mathrm{~cm} \mathrm{x}$ $3 \mathrm{~cm}$. It was also determined before design began that the target would be a water-cooled solid since the space required for the double walled mercury target container is incompatible with a compact neutron source.

Guide performance improvements are based on employing the latest technologies including high reflectivity mirrors, elliptical guides and precision alignment. Advanced instrument detector technologies will also be used to improve performance as discussed in the Instrument Section 3.

Because the largest gains in performance can be achieved with advanced neutron beam guides and instrument detectors, STS will work to advance the state-of-the-art in these areas as described in the Instrument Section 3. On the other hand, the STS target station design is anchored in the operating experience and proven component designs of the First Target Station (FTS) and other accelerator-based facilities around the world. 


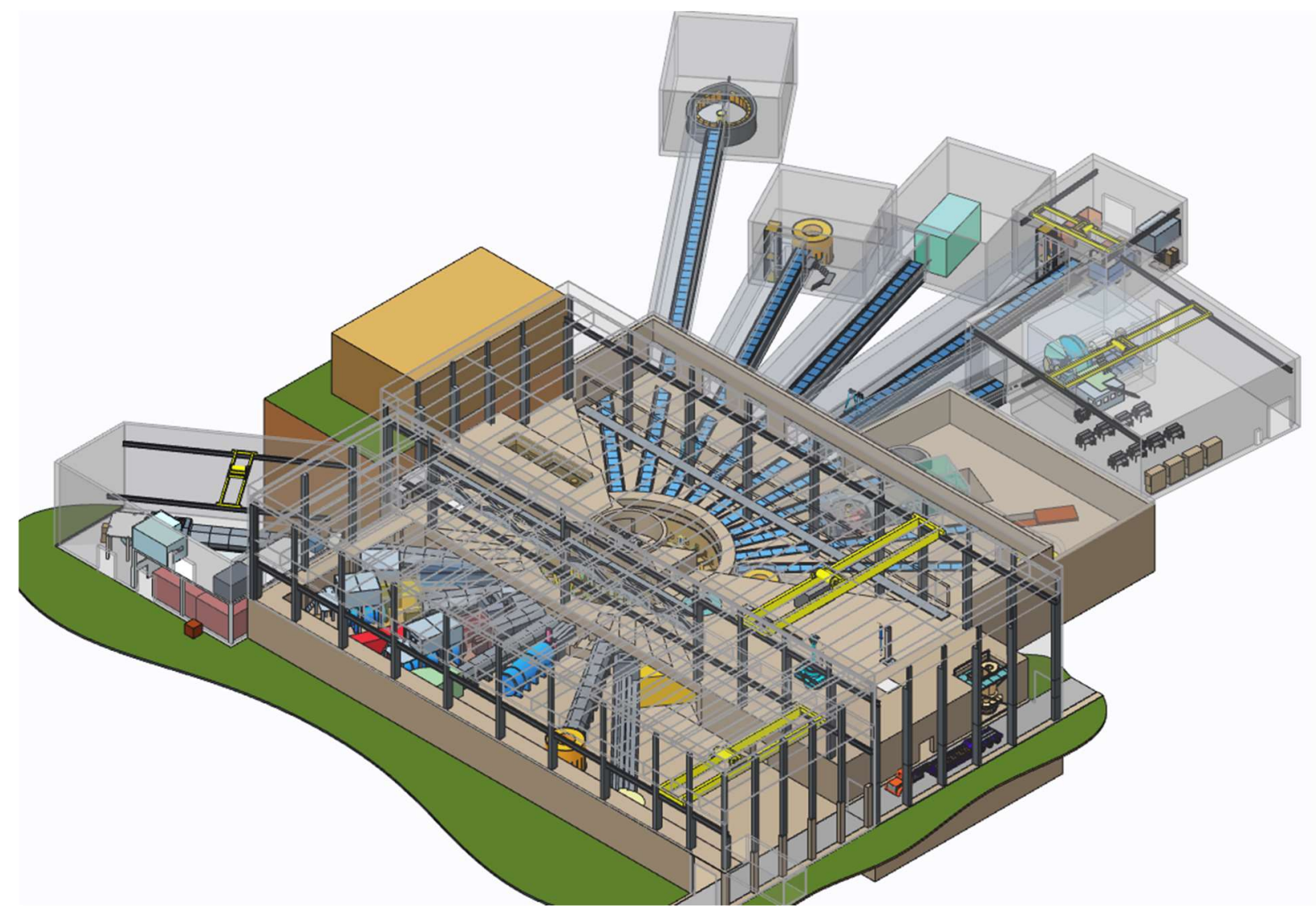

Figure 5.1. STS Target Facility including proposed instruments.

\subsubsection{Design Guidelines}

Operating experience at many facilities has provided lessons that have been applied to the design of the STS. Foremost among these is that the facility concept will be focused on providing the best performance possible for the initial suite of instruments. For example, to make room for 'short' instruments the angle between neighboring long beam lines may be increased. Special features in the target monolith and bunkers to provide for unique beam guides will also be accommodated.

Development of new and innovative instrument technologies is a fundamental requirement for the STS. On the other hand, except for future moderator technologies, significant performance gains are not feasible in the source. Therefore, to the maximum extent possible, the target station will be based on proven components and systems. This approach includes adapting the FTS philosophy of replacing activated components rather than providing shielded facilities to make repairs.

In keeping with the desire to minimize target station system and component development, STS will incorporate aspects of many systems successfully developed for FTS, including the utilities systems, cryogenic systems, remote tooling and waste handling systems. Some of the remote handling tooling and equipment designs will also be shared between the facilities. In addition, STS can take advantage of operator familiarity and incorporate changes and features to further improve performance.

STS will have, at most, eight instruments at startup. To maximize experimental productivity, the target station design facilitates installation of future instruments with minimum impact on existing operations. 
Taken together, the underlying philosophies applied to the design of STS closely follow value engineering principles. The benefits in reduced design, development and implementation can be seen throughout the system. In Section 5.6 some important examples of value engineering are highlighted. For example, a hazards analysis [3] was begun at the earliest practical stage of design to minimize engineering rework. This effort was aided by prior SNS safety studies [4] and the operating experience of the STS design team since the FTS began operation.

Finally, it should be noted that STS will be devoted solely to neutron scattering science. Alternative materials testing, component development or additional particle physics experiments are not included in the design.

\subsubsection{Accelerator Interface}

Principle target station engineering design parameters have been provided by the SNS accelerator, neutronics and conventional facilities groups. Table 5.1 provides a comparison of the STS proton beam on target with other accelerator-based neutron facilities worldwide.

Table 5.1. Accelerator characteristics of various neutron scattering facilities.

\begin{tabular}{|c|c|c|c|c|c|c|c|c|c|}
\hline Parameter & STS & $\begin{array}{l}\text { FTS } \\
\text { (SNS) }\end{array}$ & $\begin{array}{l}\text { FTS Up- } \\
\text { grade } * * *^{* *}\end{array}$ & $\begin{array}{l}\text { Luan } \\
\text { MKIII }\end{array}$ & $\begin{array}{l}\text { ISIS } \\
\text { TS1 }\end{array}$ & $\begin{array}{l}\text { ISIS TS1 } \\
\text { Upgrade* }\end{array}$ & $\begin{array}{l}\text { ISIS } \\
\text { TS2 }\end{array}$ & CSNS** & ESS \\
\hline Energy $(\mathrm{GeV})$ & 1.3 & 1.0 & 1.3 & 0.8 & 0.8 & 0.8 & .8 & 1.6 & 2 \\
\hline Frequency $(\mathrm{Hz})$ & 15 & 60 & 60 & 20 & 40 & 50 & 10 & 25 & 14 \\
\hline Power (kW) & 700 & 1400 & 2000 & 160 & 160 & 440 & 32 & 120 & 5000 \\
\hline $\begin{array}{l}\text { Average current } \\
\qquad(\mathrm{mA})\end{array}$ & 0.538 & 1.4 & 1.5 & .2 & .2 & .55 & .04 & .063 & 2.5 \\
\hline $\begin{array}{c}\text { Protons/pulse } \\
\left(10^{14}\right)\end{array}$ & 2.24 & 1.46 & 1.60 & .624 & 0.31 & .69 & .25 & .16 & 11.1 \\
\hline $\begin{array}{c}\text { Target } J \text { peak } \\
\left(\mu \mathrm{A} / \mathbf{c m}^{2}\right)\end{array}$ & 12.8 & 17.5 & 19.2 & 14.1 & 12 & 38.9 & 25 (est) & .8 & 56 \\
\hline $\begin{array}{c}\text { Peak target } \\
\text { Protons } / \mathbf{m}^{2} / \text { pulse } \\
\left(10^{16}\right)\end{array}$ & 5.33 & 1.82 & 2.0 & 4.4 & 1.87 & 4.86 & 15 (est) & .2 & 25.0 \\
\hline
\end{tabular}

* Planned; ** Predicted; ***before STS startup

The profile of the proton beam incident on the target is shown in Figure 5.2. The accelerator focuses $90 \%$ of the beam within an ideal $4 \mathrm{~cm} \times 7.5 \mathrm{~cm}$ envelope with a minimum amount of peaking to reduce stress in the target assembly. The remaining $10 \%$ of the beam is contained in a rectangular boundary around the ideal area. This beam profile was consistently used in the neutronics and engineering studies throughout the design process. 


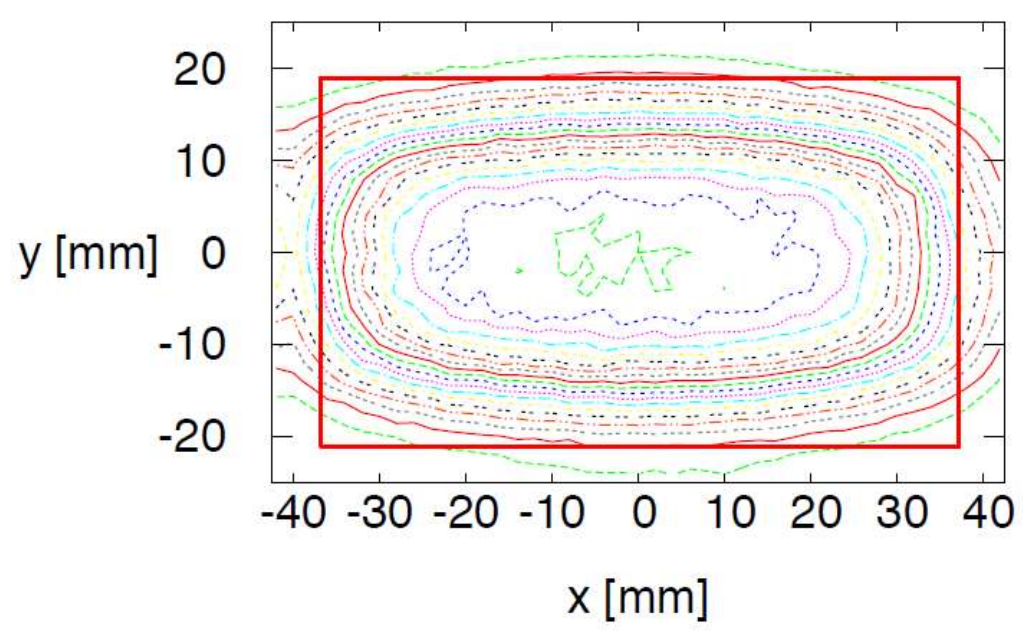

Figure 5.2. STS proton beam profile (Peak is $\left.190 \mathrm{~mA} / \mathrm{m}^{\wedge} 2\right)$, at $700 \mathrm{~kW}$.

\subsubsection{Design Basis Neutronic Analysis}

Neutronics analysis and engineering design were closely coupled using the Direct Accelerated Geometry Monte Carlo (DAGMC) Toolkit integrated into MCNP6, which is developed at the University of Wisconsin-Madison. The DAGMC toolkit is used to convert the ProE Creo engineering model into a high fidelity neutronics model as shown in Figure 5.3, which can be analyzed with MCNP6. The model was sized to encompass a $3 \times 3 \times 3 \mathrm{~m}^{3}$ cube-shaped section of the target-reflector-monolith intended to bound the region which requires active cooling during beam-on operations. This provides basic engineering design information used to size the cooling water distribution system and the type of cooling required for each of the cooled components.

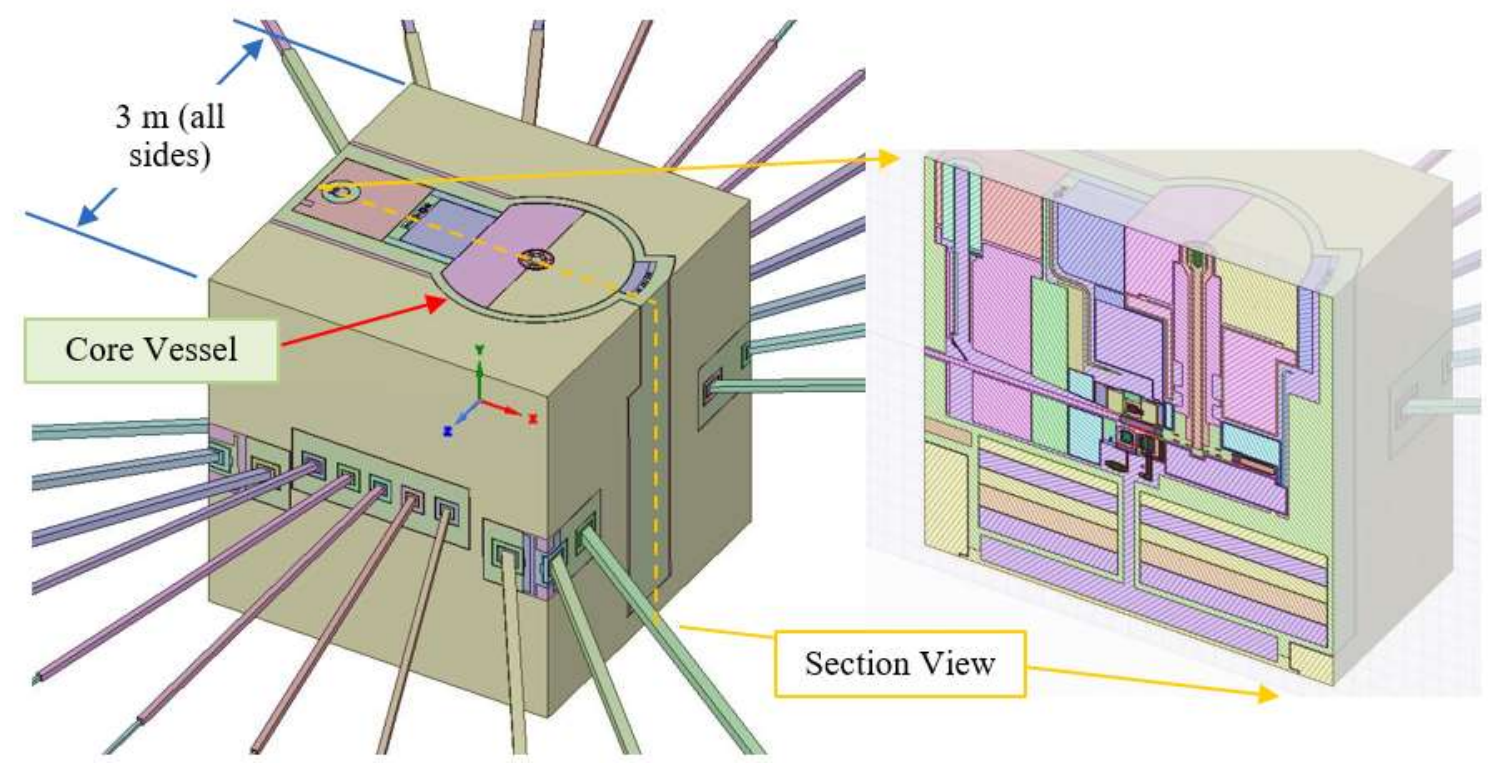

Figure 5.3. Neutronics model obtained by automatic conversion of the ProE Creo mechanical model with DAGMC toolkit.

Figure 5.4 and Figure 5.5 depict heating rates around the rotating target, for the $700 \mathrm{KW}$ operation (15 $\mathrm{Hz}$ ). The two contour lines depict the $0.01 \mathrm{~W} / \mathrm{cm}^{3}$ and $0.001 \mathrm{~W} / \mathrm{cm}^{3}$ heating rate levels. In general, all 
components inside the $0.001 \mathrm{~W} / \mathrm{cm}^{3}$ boundary are assumed to require active cooling. Within the 0.01 $\mathrm{W} / \mathrm{cm}^{3}$ boundary, components are assumed to require contact water cooling; namely, contained inside vessels containing circulating water.

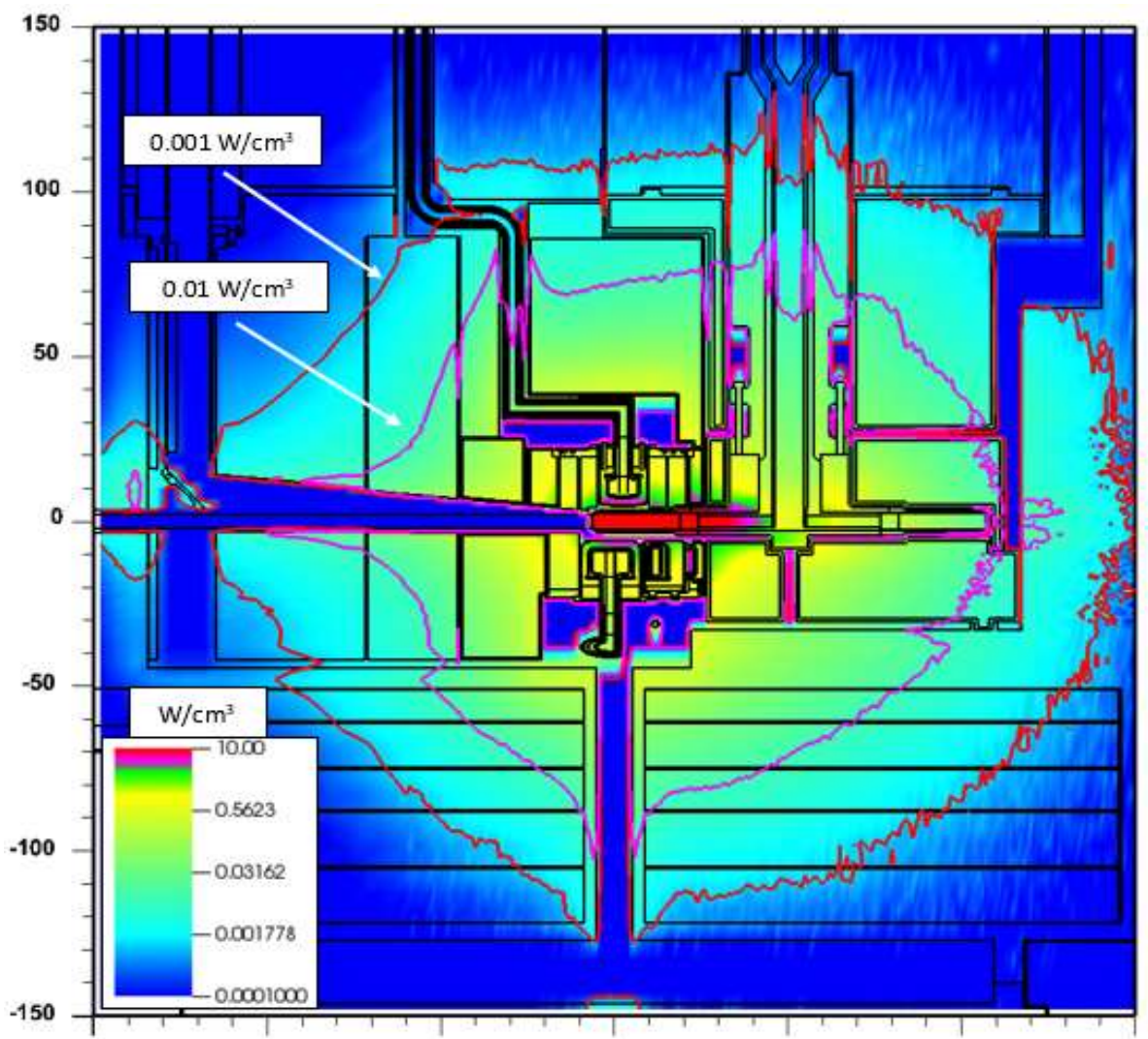

Figure 5.4. Heating rates in $\mathrm{W} / \mathrm{cm} 3$ around the target at $700 \mathrm{KW}$ operation $(15 \mathrm{~Hz})$. Vertical section along the proton beam direction is shown. Values within rotating components are not valid. 


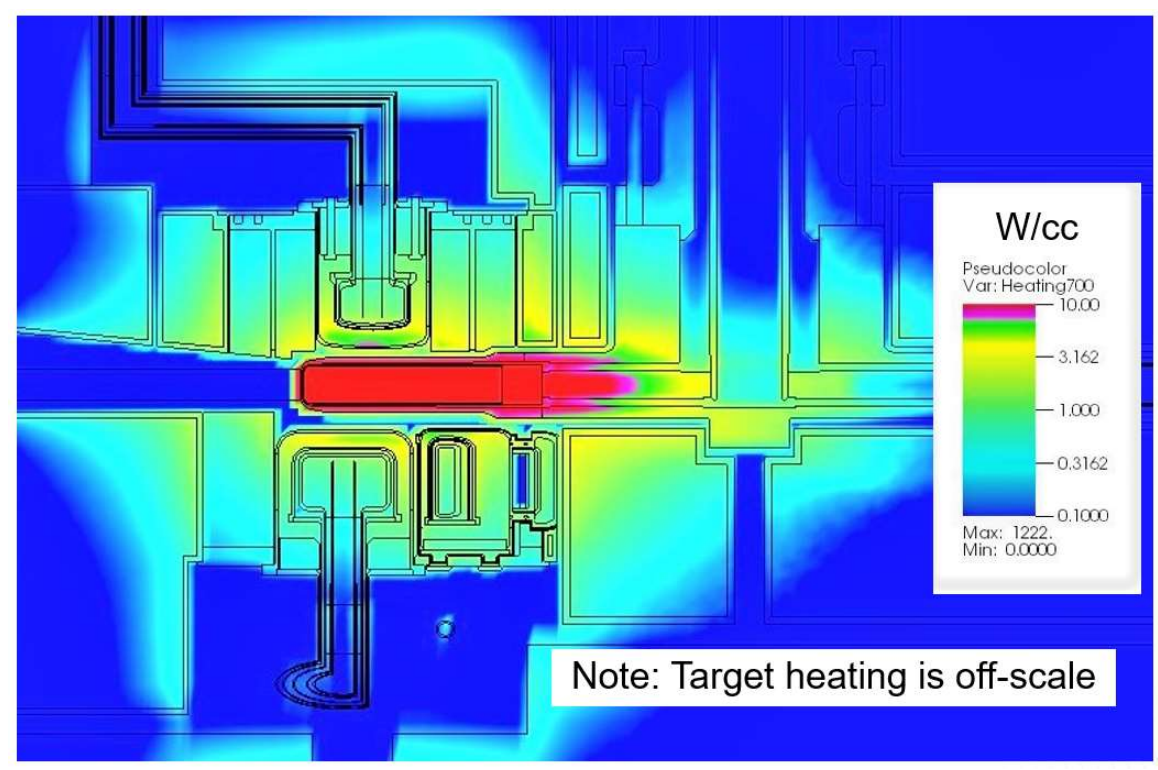

Figure 5.5. Heating rates in $\mathrm{W} / \mathrm{cm}^{3}$ near the target at $700 \mathrm{KW}$ operation $(15 \mathrm{~Hz})$. Vertical section on proton beam horizontal center is shown. Values within rotating components are not valid.

The high-fidelity model was also used to determine the integrated heat loads in the target-reflector assembly components. The maps of radiation damage displacements-per-atom (dpa) rates were obtained by folding the neutron and proton fluxes calculated with detailed MCNP6 model with the dpa crosssection for steel or aluminum. The 5000 hours of operation of beam on the target was assumed to determine the dpa/year values. The results are displayed in Figure 5.6 for steel (SS-316) and Figure 5.7 for aluminum. The dpa values are used to help determine the operating life of the components located close to the center of the monolith such as the moderator-reflector assembly and shielding near the target. 


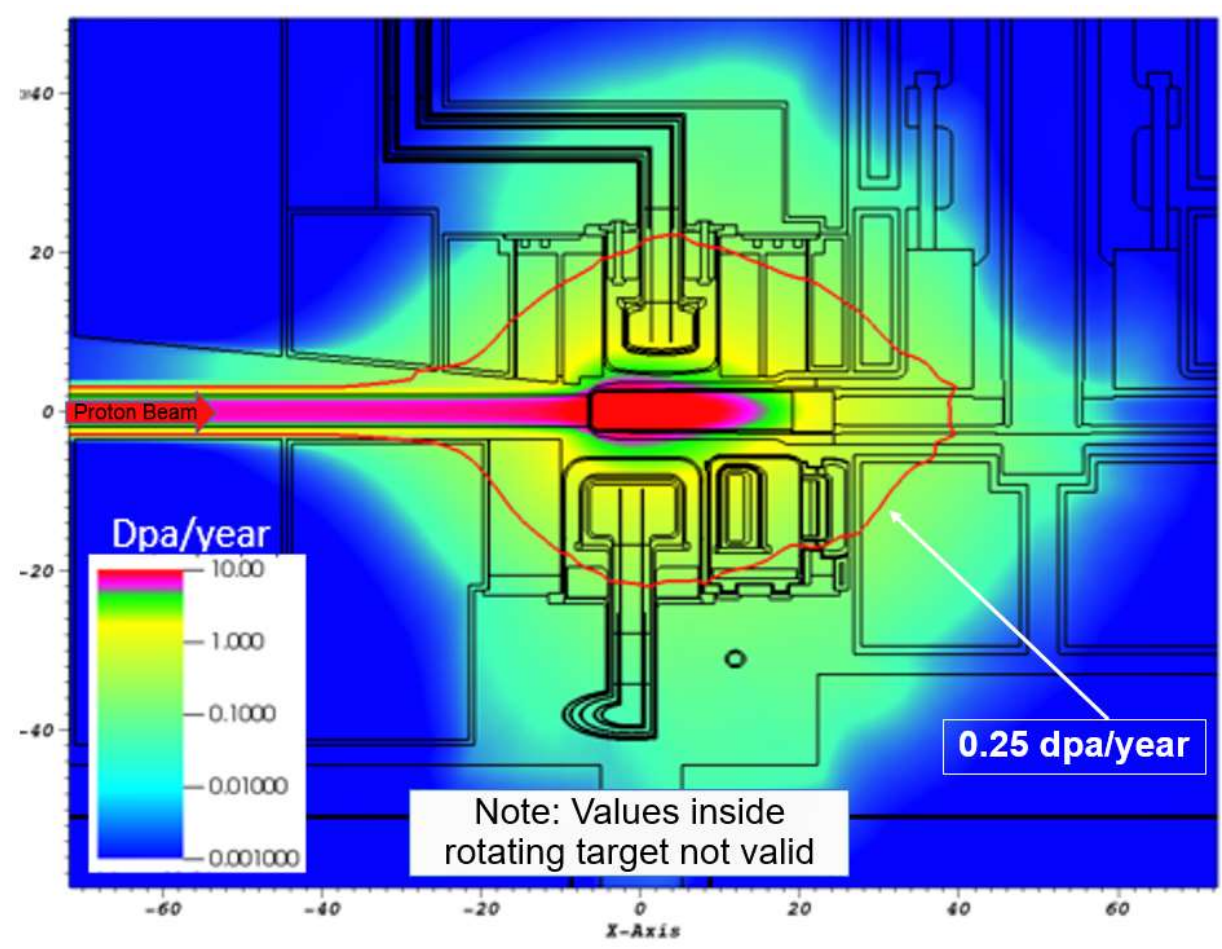

Figure 5.6. Total $(n+p)$ dpa per year in stainless steel; 5000 hours per year of beam-on-operation is assumed.

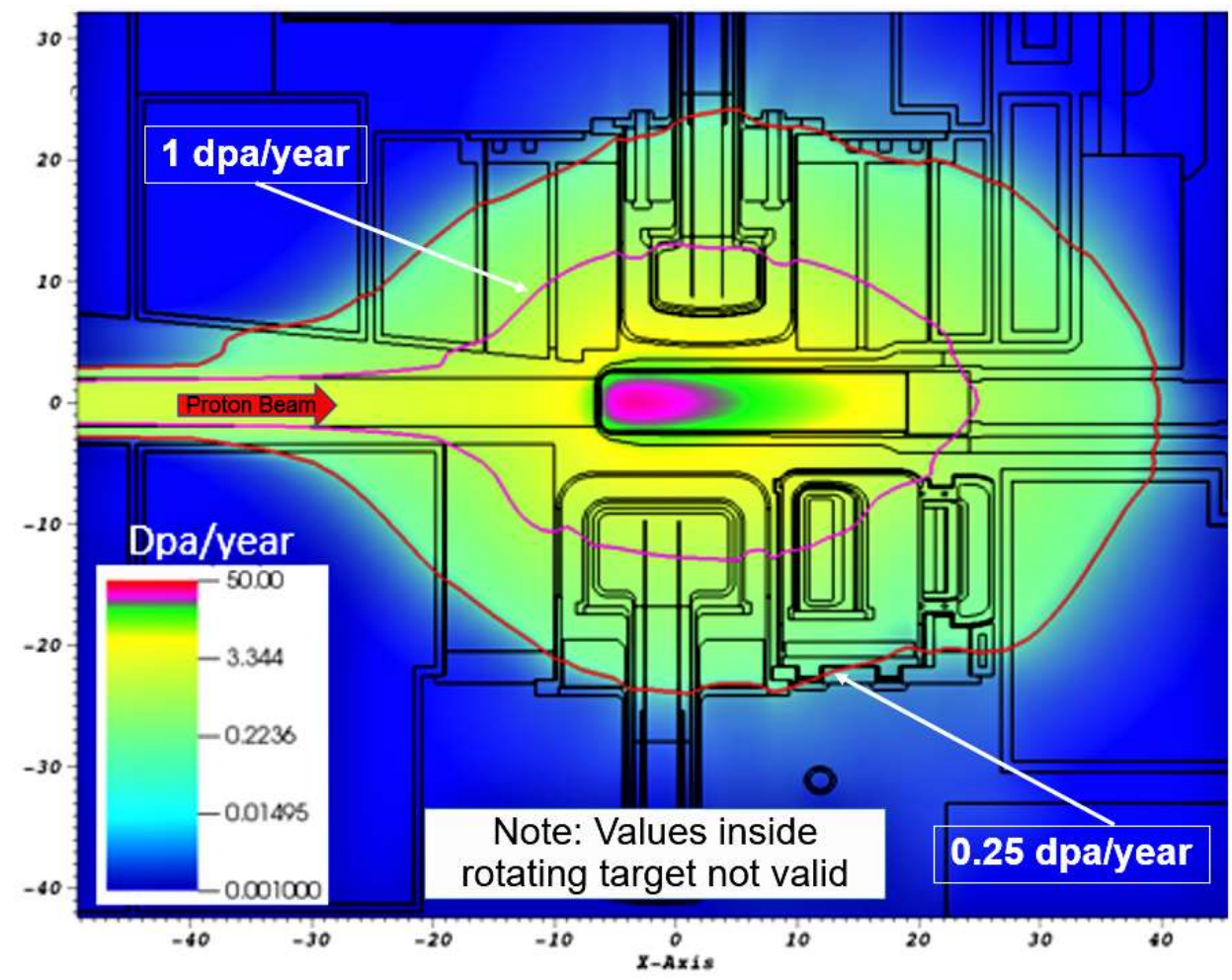

Figure 5.7. Total $(\mathbf{n}+\mathbf{p})$ dpa per year in aluminum. 5000 hours per year of beam-on-operation is assumed. Aluminum components. 


\subsection{TARGET AND INSTRUMENT FACILITY CONFIGURATION}

Consideration of a Second Target Station was always part of the original SNS concept, so the site effectively accommodates the new target station and utilities. The STS target building is positioned to match the proposed instrument suite, which is naturally divided into "short" $(<60 \mathrm{~m})$ on one side and "long" instruments (up to $100 \mathrm{~m}$ ) on the other side. The short side of the facility is also referred to as the "40-meter or 40M" side since that's the approximate width of that instrument hall. The horizontal section view of the building at the proton beam level (Figure 5.8) shows the neutron beam line bunkers on both sides of the target monolith. Neutron beam line choppers, precision guide alignment devices, and other near-monolith components are located within bunkers to provide more space and better personnel access than individually shielded lines. The section view also shows the shielded process utility bays near the proton beam line, the truck bay, waste transfer bay and the instrument support area. The instrument support area will primarily house the sample environment equipment and preparation activities. The location of this area is important since it provides direct access to all beam lines. This area is the only section of the target building occupied by instrument scientists; all other areas of the building will be accessible only by SNS operating and maintenance personnel.

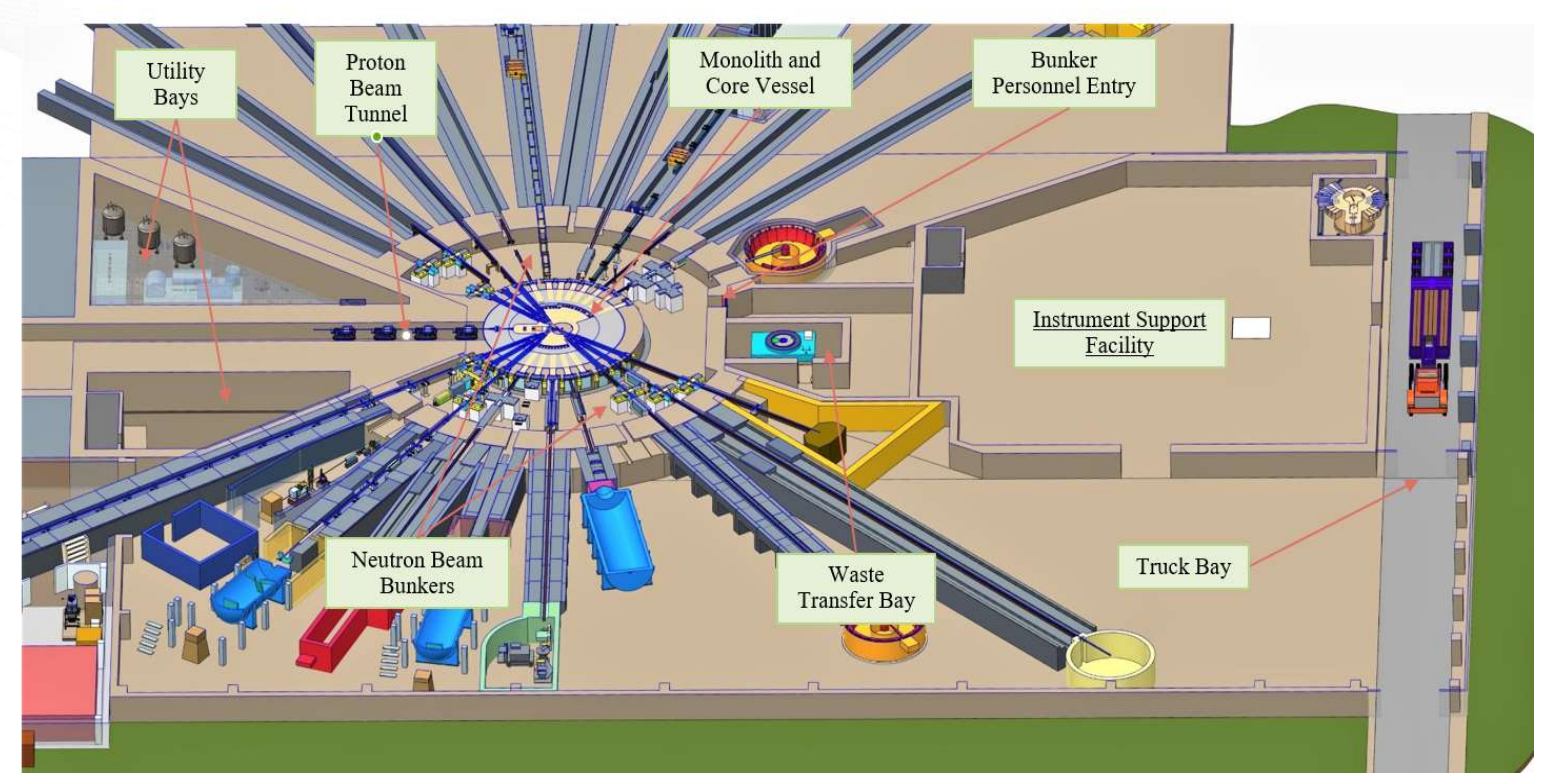

Figure 5.8. Target building horizontal section at proton beam level.

The longitudinal section view of the target building (Figure 5.9) shows the horizontal proton beam terminating at a rotating target in the center of the monolith. This view shows the three levels in the building including the 50-ton bridge-crane-serviced high bay. The target drive bunker above the monolith shields personnel during beam-on operations but provides access during maintenance shutdowns via an airlock and shield door at the high bay level. Building extensions provide high bay crane access to the long lines and truck bay.

The 40M instruments are housed in a single span hall beside the target building (lower portion of Figure 5.8). The hall has a 30-ton bridge crane with access to all the instruments and the truck bay. The floor is located $1.82 \mathrm{~m}$ below the proton beam centerline to provide space to install most detectors, although it is assumed that pits may be required for some detector arrays. All instruments in the $40 \mathrm{M}$ building will be responsible for all of their infrastructure, including beam line shielding. 


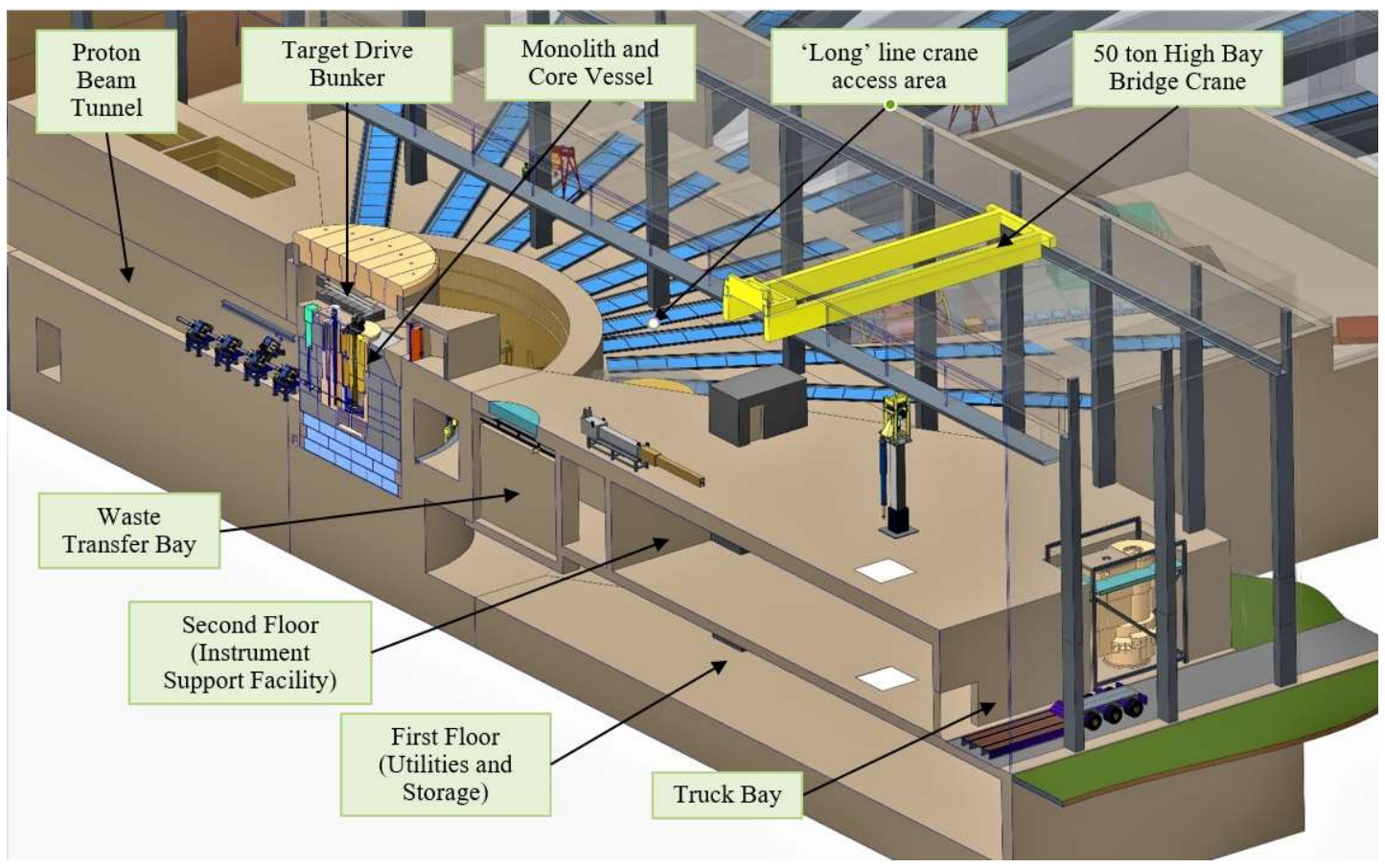

Figure 5.9. Target building vertical section view along proton beam line.

Crane access to the long instruments has been incorporated through a Crossover Bay in the target building (Figure 5.10). There is no floor in the high bay over this portion of the long instrument hall, which gives allows heavy components to be lifted from the truck bay, over the high bay, and into the instrument hall. No such open floor area exists on the $40 \mathrm{M}$ side of the high bay because a separate crane covers that entire instrument hall, and there is a desire to have separate ventilation systems between the $40 \mathrm{M}$ instrument hall and the target building. Minimizing cost of the target building requires minimizing its width, so the crossover bay only provides crane coverage over a few meters of the long beam line trenches.

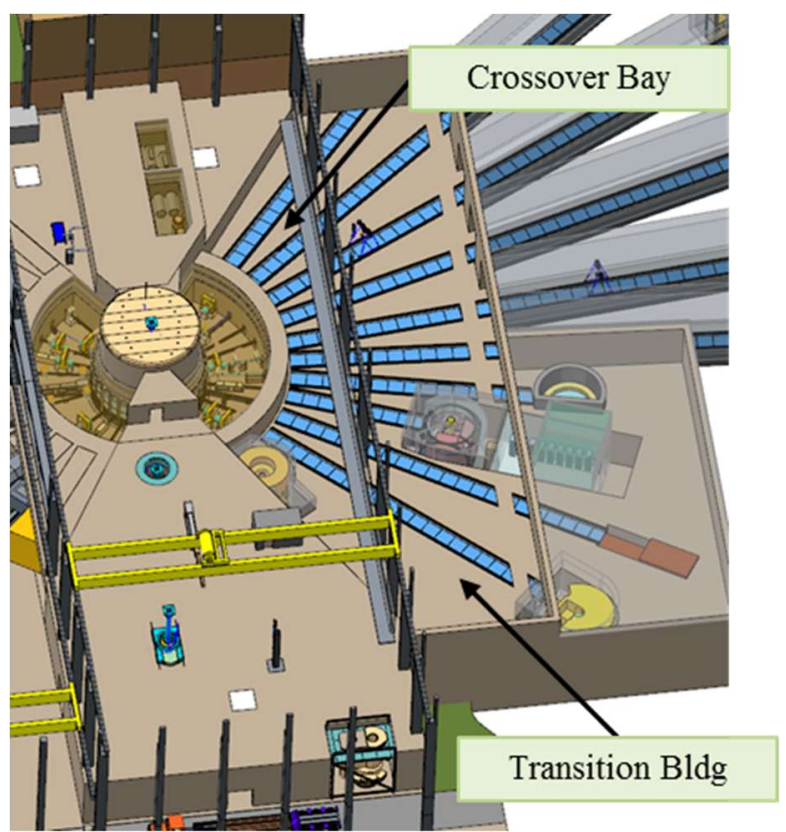

Figure 5.10. Long instrument side of target building. 
A second space referred to as the Transition Building, also shown in Figure 5.10, extends far enough along the trenches to provide adequate separation between them for construction of individual enclosures outside the building. There is no overhead crane coverage planned for this area. Instead, each trench will have tracks along its entire length to accommodate a portable gantry hoist. These hoists will be shared among the beam lines and will be used to lift and transport trench shield blocks, choppers, and other beam line components between the crossover bay and the long instrument buildings. More detail of the trenches is given below and in Section 5.4.5. The transition building will also provide operating and storage floor space since the need to access beam line equipment has proven to be infrequent during FTS operations.

The choice of neutron beam line bunkers (Figure 5.11) rather than stacked shielding was driven primarily by the desire to locate accessible beam guide components as close to the neutron source as physically possible. In horizontally-arrayed beam line configurations, personnel access to beam line components is limited by shielding and angular spacing between adjacent beam lines. While only passive optical components are usually located between the shutters and moderators, there is a desire to place choppers and other beam line components which require maintenance as close to the source as possible. Open bunkers (with local shielding of specific components) offer much better access to these components than is possible with completely shielded beam lines. In addition, the installation of future beam lines is significantly improved by the ability to physically access this area rather than requiring totally remote beam line installation and alignment.

The STS neutron bunkers have inner and outer radii approximately 5.5 meters and 11.5 meters from the moderators; this distance is primarily driven by the length of the equipment required to handle the monolith inserts that are installed into the monolith structure. It is currently assumed that the bunker walls and ceiling will be roughly 2 meters thick high density concrete with exceptions for special features.

Removable roof panels and a floor level personnel entrance will provide beam-off crane access. Personnel can enter either through the ceiling using purpose-built ramps or through a door at the back of the bunkers. Providing personnel access to the bunker during these beam-off maintenance periods, even after years of facility operation, is a fundamental design driver for the facility.

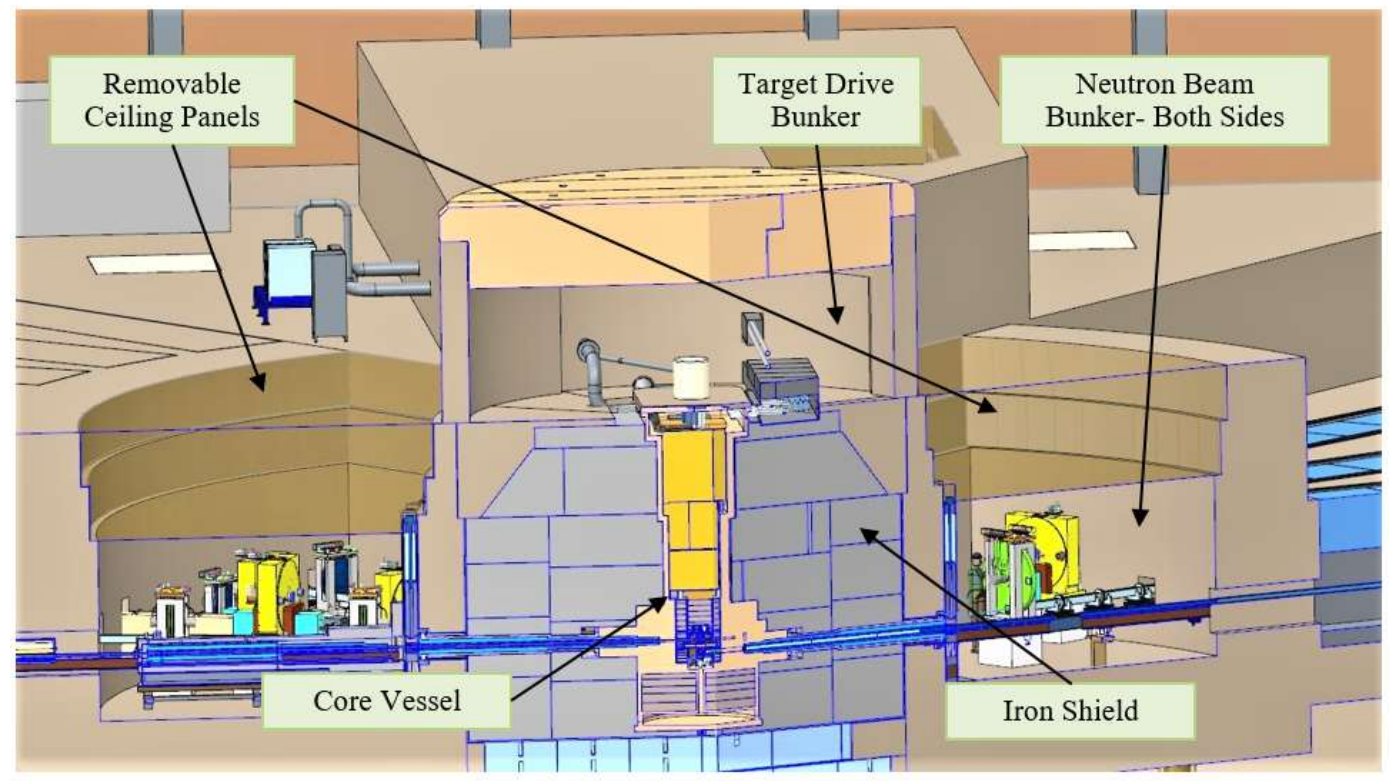

Figure 5.11. Target building section view showing neutron beam line bunkers.

The "long" instrument side of STS includes a mix of beam line lengths ranging from $<20 \mathrm{~m}$ to $100 \mathrm{~m}$; therefore, housing the long side beam guides was recognized early in the facility design process as a key 
concern. To determine the most cost effective approach, a trade-off study [5] was performed with the assistance of an outside Architect-Engineering (AE) contractor. The option of constructing a single large building covering all possible instrument positions was eliminated early due to high up-front costs, operating difficulties (e.g., crane coverage) and functional limitations (e.g., ventilation, lighting, emergency egress, etc.). This resulted in the current arrangement in which the long (generally over $40 \mathrm{~m}$ ) instruments will be located outside the target building in stand-alone structures. In all cases, it was determined that the target building must be wide enough to provide direct bridge crane access to some portion of the beam lines to support experimental and maintenance operations.

Three stand-alone building options for long line coverage were evaluated in the trade-off study; above ground, trenches, and tunnels (Figure 5.12). Design requirements included accommodation for covered, continuous walkways for instrument scientists and sample environment technicians; shield handling efficiency; utilities routing from the target building to the instrument buildings, thermal control of the beam guide, and cost.

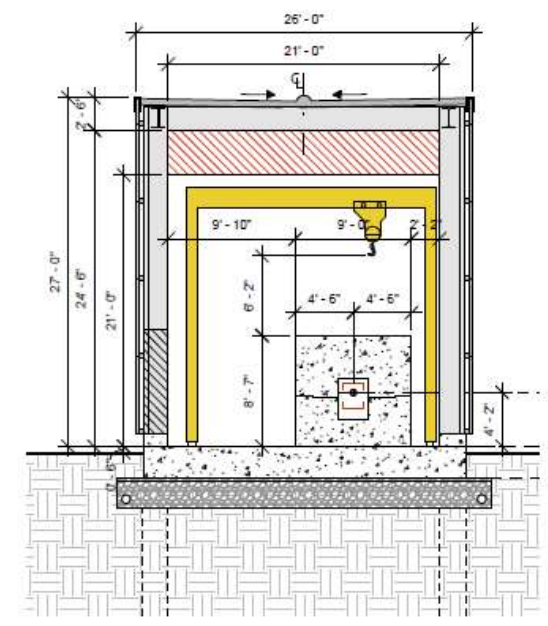

Above Grade
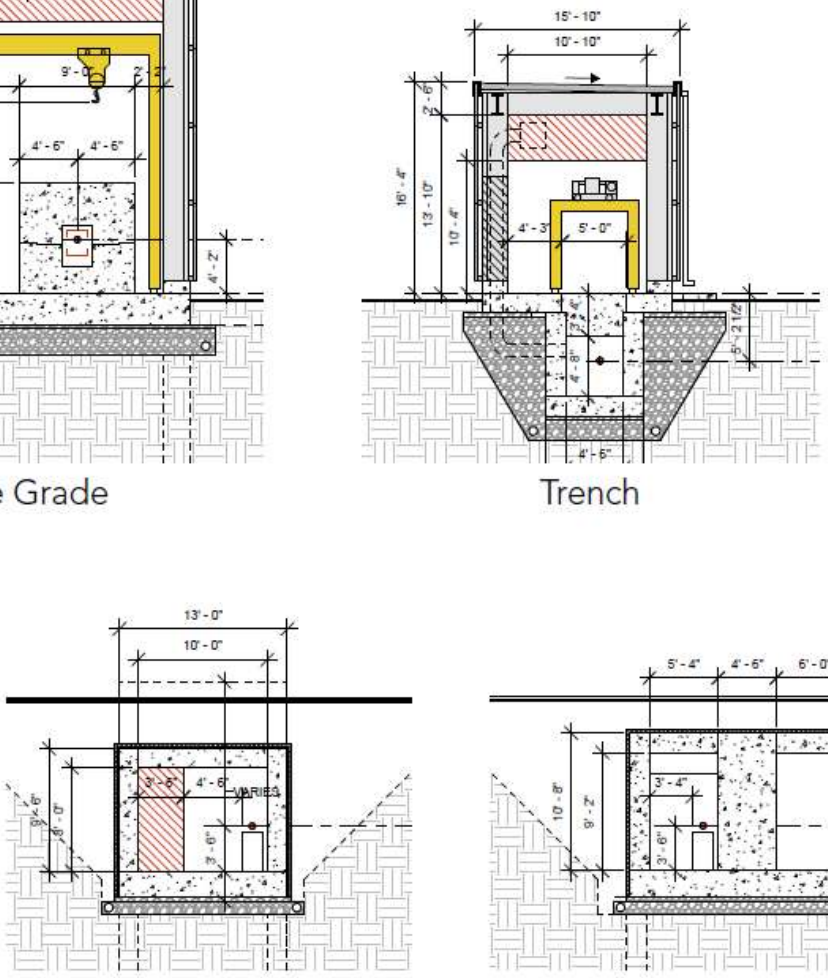

Tunnel B

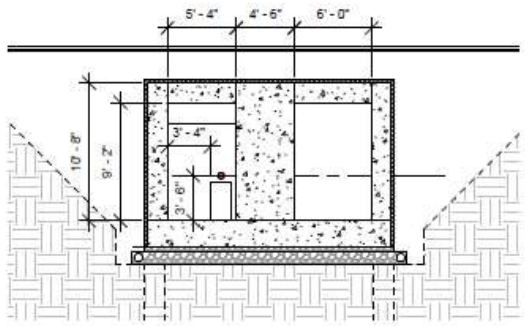

Double Compartment Tunnel

Figure 5.12. Long beam line enclosure options.

A weighted criteria evaluation of the options was performed, and the trench option was selected because it received the highest technical score and was also the lowest cost. Compared to typical above grade buildings, trenches save costs in bulk shielding since its trench shape also serves as shielding and only trench cover shields must be fabricated. Potential disadvantages of trenches include the possible difficulty of fitting large choppers and providing adequate space for personnel to maintain guide optics. While the access issues associated with trenches are related to relatively infrequent maintenance operations and are 
amenable to design solutions, the higher-cost above-ground and tunnel options presented continuous operating difficulties since personnel and sample environment equipment were required to be continuously moved over neighboring lines. It should also be noted that the trenches provide a significant amount of working and storage space inside the target building once beam lines are installed. Figure 5.13 shows a view of the upstream ends of the trenches just outside the bunker. It is anticipated that the eleven trenches within the target facility and transition building will be built as part of the initial facility construction along with those exterior trenches which are part of the initial instrument suite.

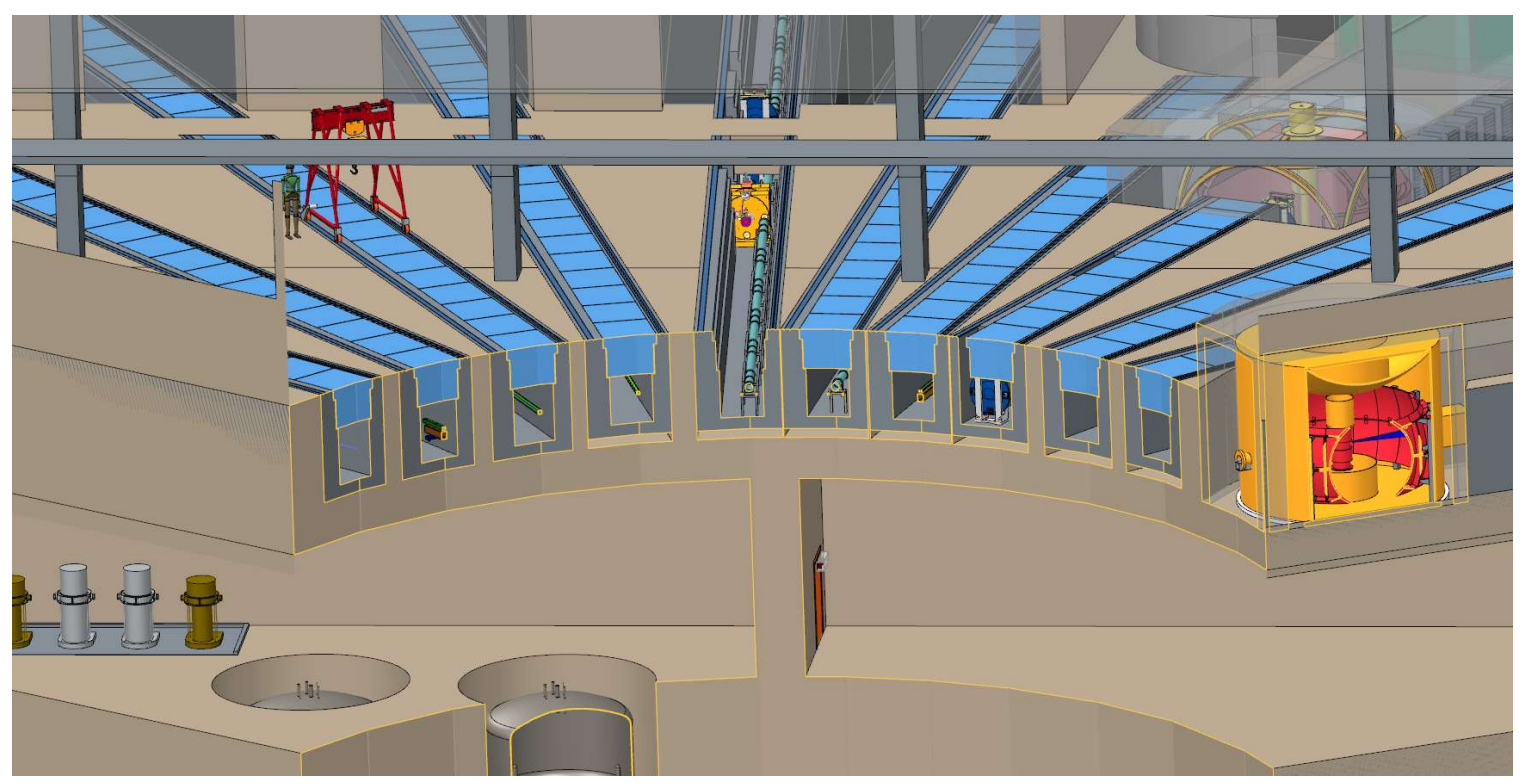

Figure 5.13. Long beam line trenches viewed from just outside the outer bunker wall. Removable trench shield covers (shown in blue) are $1 \mathrm{~m}$ thick.

\subsection{TARGET SYSTEMS}

\subsubsection{Target Monolith}

The target monolith (Figure 5.11) encloses the high radiation area of the STS target facility. A 9-meter diameter cylinder of iron shielding surrounded by an approximately one-meter thick, high-density concrete (HDC) wall protects personnel and equipment. A core vacuum vessel in the center of the monolith encloses the functional target system components (Figure 5.14). The shielded "Target Bunker" above the monolith provides access to the target drive and enables vertical handling of spent activated component assemblies. The $4.2 \mathrm{~m}$ height of the shielding between the target and the target bunker is based on the operating experience at FTS where virtually no activation of components above this level has occurred after 11 years of operation. Eleven neutron beam lines project through both sides of the monolith into the "Beam Line Bunkers." 


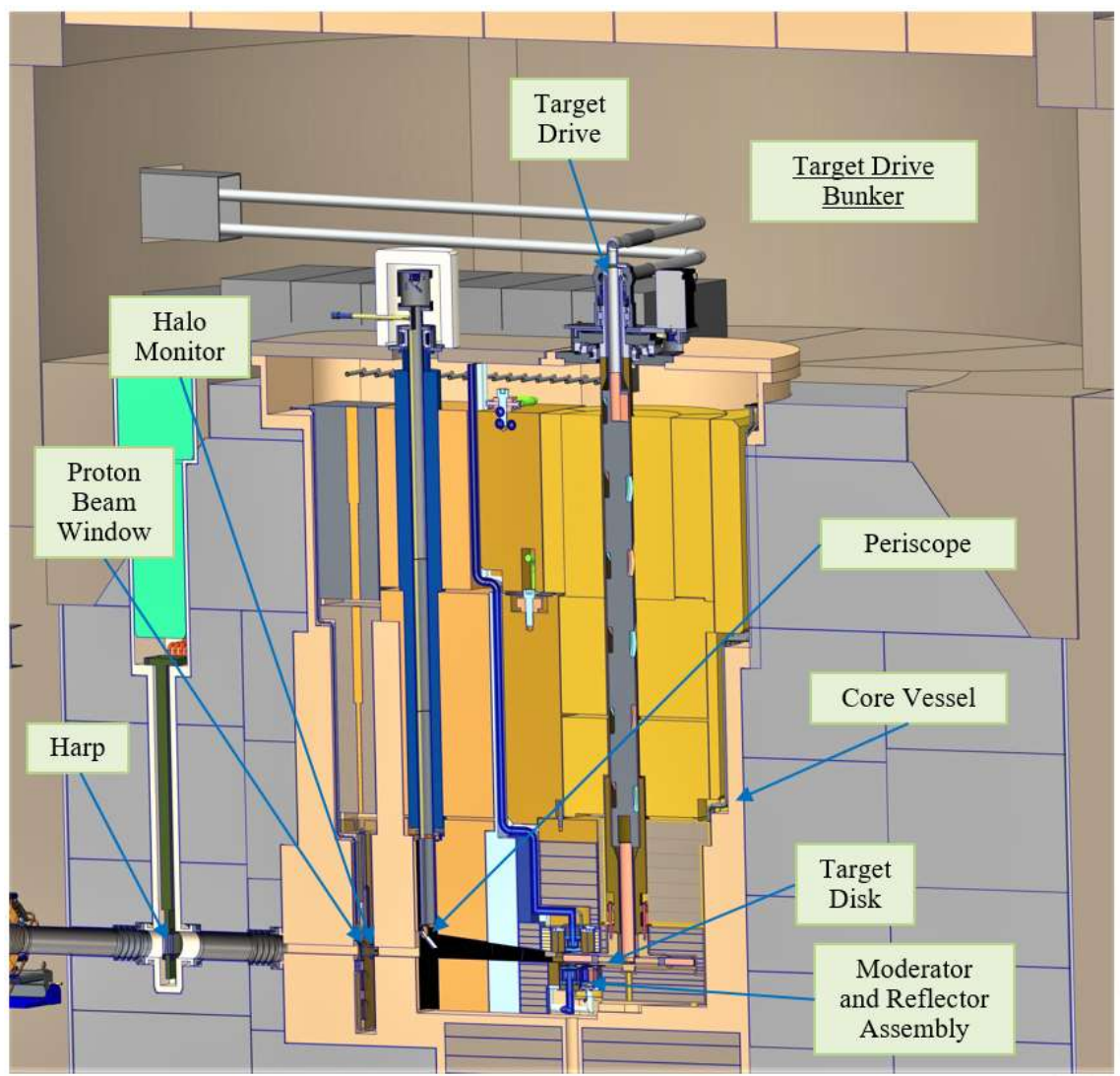

Figure 5.14. Section view along proton beam line of target station monolith and core vessel.

The location of key components along the proton beam line is shown in Figure 5.15. Other than the moderator-reflector plug, remote handling considerations dominated the proposed layout. This can primarily be seen in the spacing of the removable shield blocks (Figure 5.14).

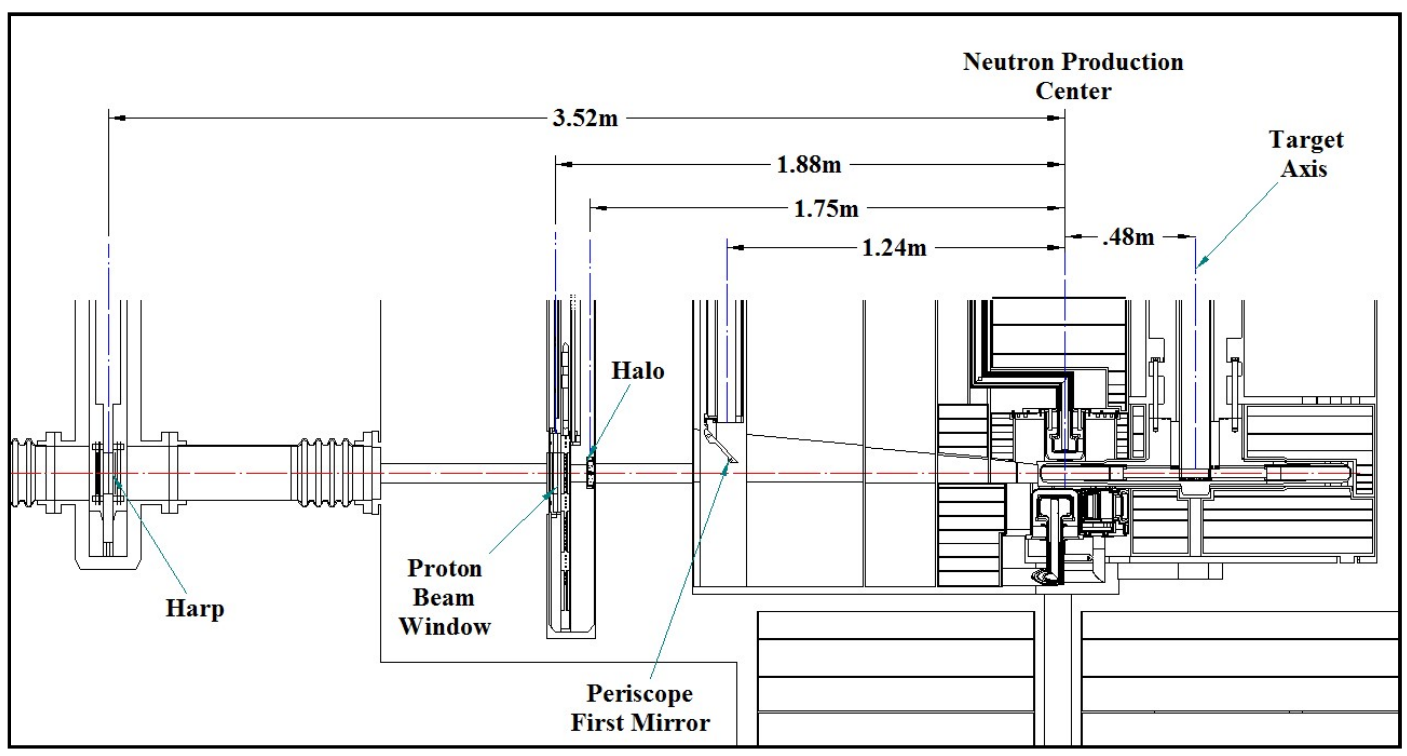

Figure 5.15. Section view of core vessel showing location of primary components. 


\subsubsection{Core Vessel and Mockup}

The core vessel (Figure 5.16) serves as the machined base for placement of all the target components in addition to maintaining the vacuum operating environment near the target. The proposed vessel must be machined and installed with as-built and in-situ measurements recorded to ensure that instrument guides, proton beam, target disks and moderators are accurately aligned over the life of the facility. Installation of all the features used to locate components during final machining of the vessel interior, possibly in a single tooling set-up, is considered a reasonable objective based on the FTS vessel (Figure 5.17) fabrication experience where tolerances of less than $\pm 0.5 \mathrm{~mm}$ were achieved.

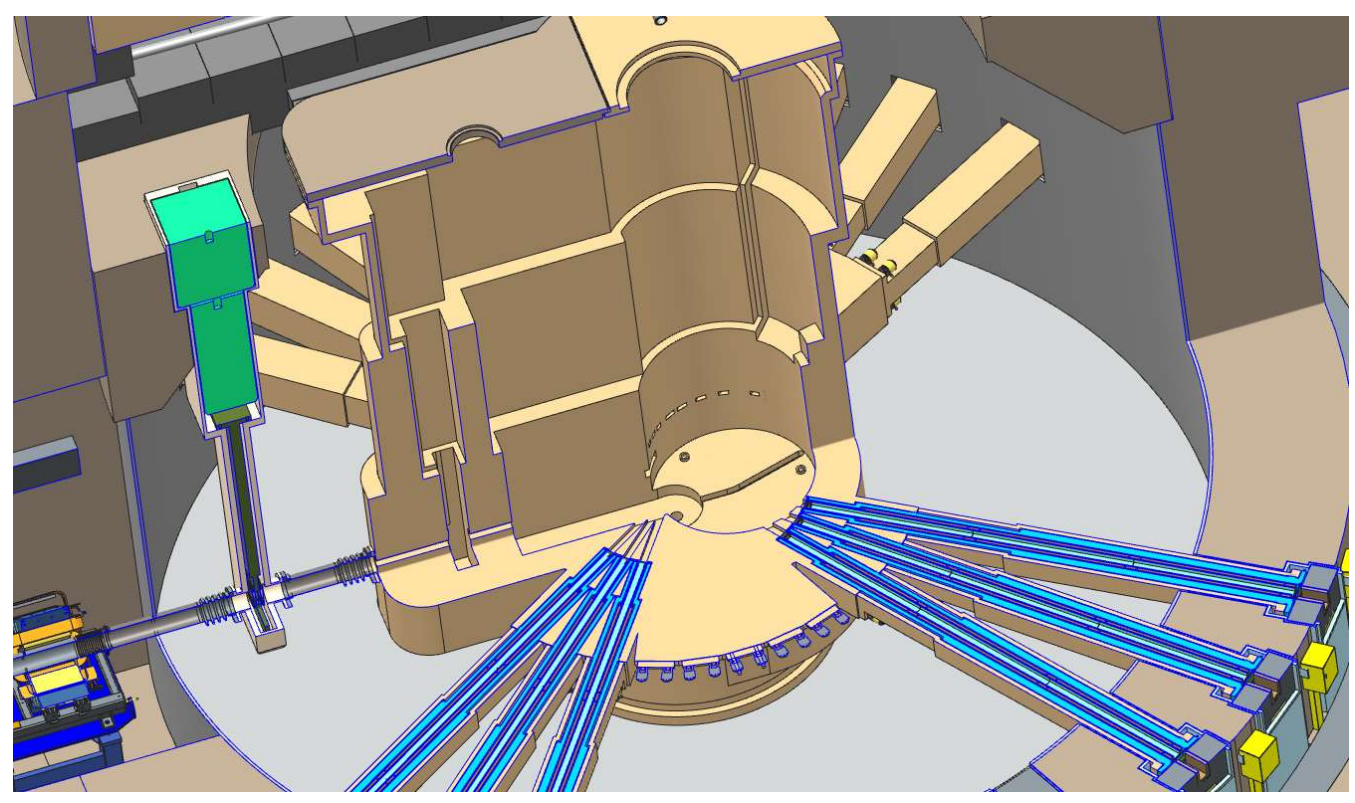

Figure 5.16. Core Vessel assembly.

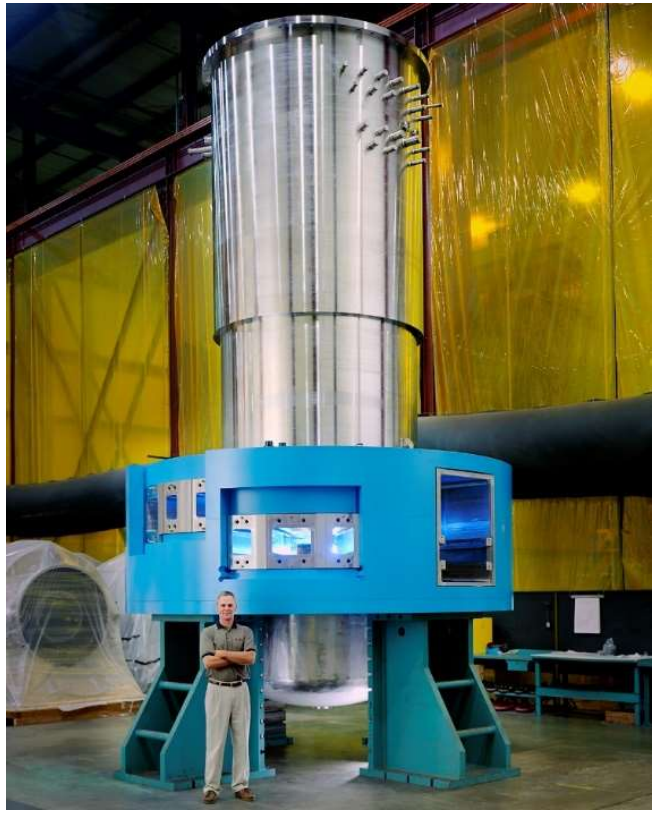

Figure 5.17. FTS core vessel assembly. 
The STS proposal includes a full-scale vessel mockup (Figure 5.18) fabricated in conjunction with the core vessel. The goal is to have the mockup match key component positioning and clearance features as accurately as possible. The stand will be used to store replacement assemblies, align new components, dry run maintenance tooling, and train maintenance personnel.

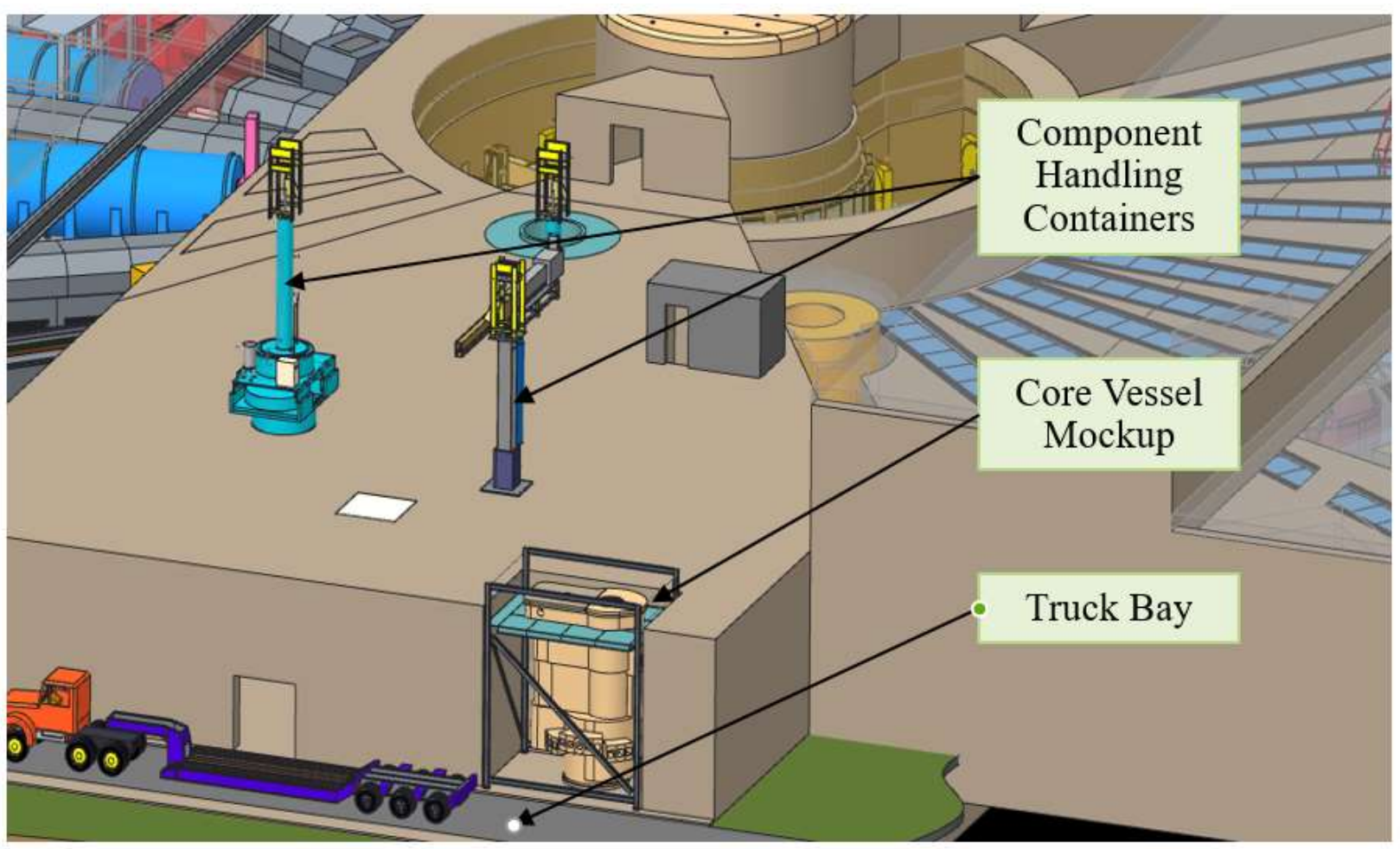

Figure 5.18. Target station mockup stand.

\subsubsection{Moderator-Reflector Assembly}

Cryogenic moderators surrounded with beryllium reflector is a baseline physics requirement. Close proximity of the moderators to the target is a corollary parameter that fits within the overall precision alignment requirement for all the compact neutron source components. The fit-up requirement will be achieved in three ways. First, the assembly is designed to be machined and assembled with a closely toleranced target gap. Second, the moderator-reflector assembly will be positioned inside the vessel on machined surfaces near the target to eliminate significant thermal motion. And, third, the relative position of the assembly will be accurately monitored using an optical periscope. Adjustment features on the assembly will allow final, vertical alignment to within $\pm 0.5 \mathrm{~mm}$ of nominal using this capability. The gap between the target and the moderators is primarily determined by the thermal expansion/contraction of the target axle and the anticipated maximum warp and machining tolerance of the disk and axle assembly.

Figure 5.19 shows the currently assumed relationship between the moderators and the target. These target to moderator gaps are currently a conservative $0.8 \mathrm{~cm}$ above and $1.4 \mathrm{~cm}$ under the disk. In the next phase of design, it is the goal of the design team to reduce the gaps by providing target alignment features, reduced manufacturing tolerances, and thermal compensation. 


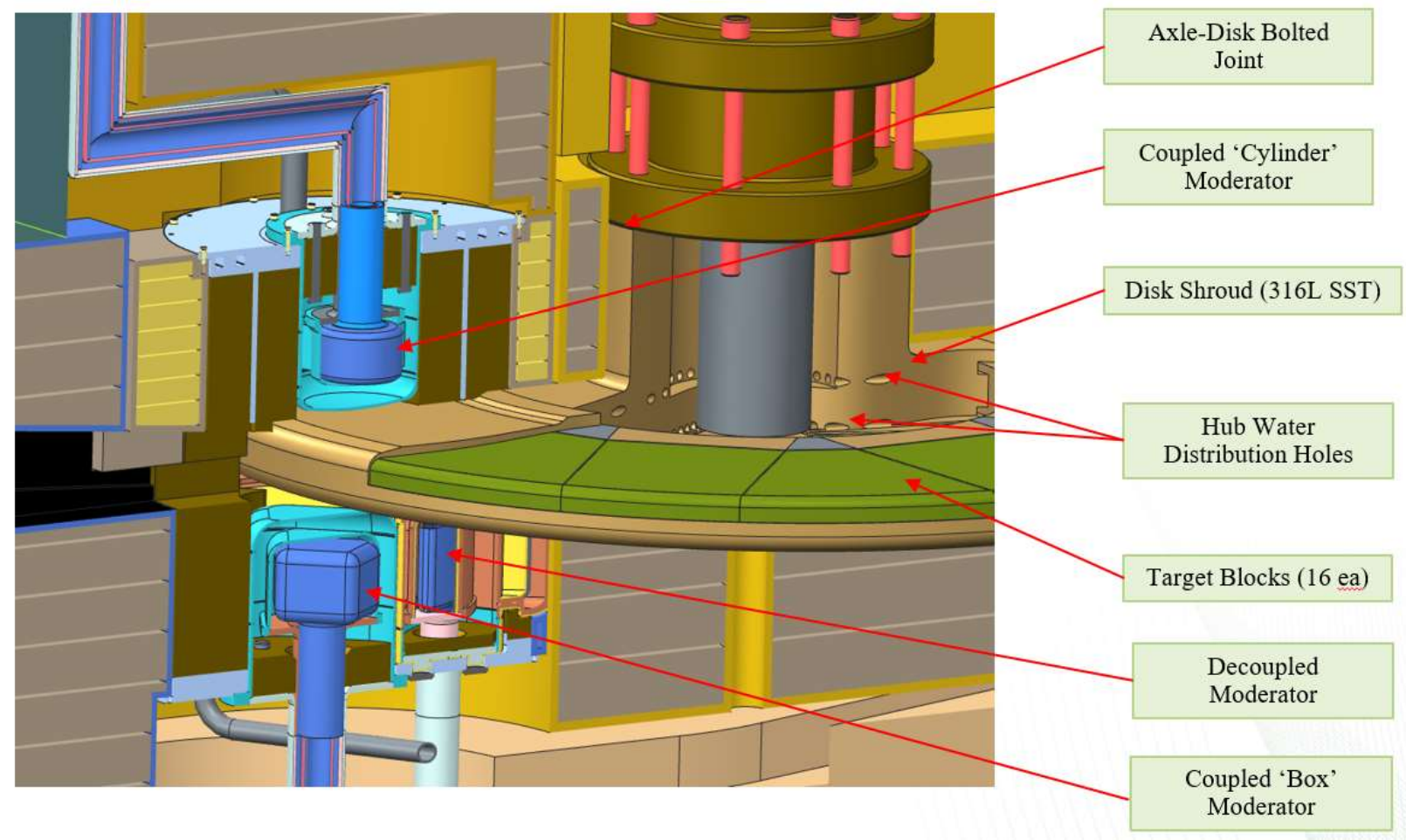

Figure 5.19. The relationship between the target and the moderators in the beam-on operating condition.

Moderator-reflector plugs have an anticipated life of 1-2 years based on the cadmium poison burn-up rate. Provisions have been made to exchange spent assemblies using the disassembly in-place technique employed successfully in the FTS (Section 5.5.2). Access to the lower moderator requires the assembly be moved horizontally approximately 0.4 meters to clear the target disk to allow vertical removal. The horizontal movement is facilitated with built-in trolleys on the plug and rails in the vessel (Figure 5.20). A mechanism to provide a small amount of vertical travel for the plug is included in the trollies to enable the load to be transferred between the trolleys and alignment pins near the level of the target. At installation, this unloads the wheels and fixes the moderator position, thus limiting vertical movement due to thermal changes. 


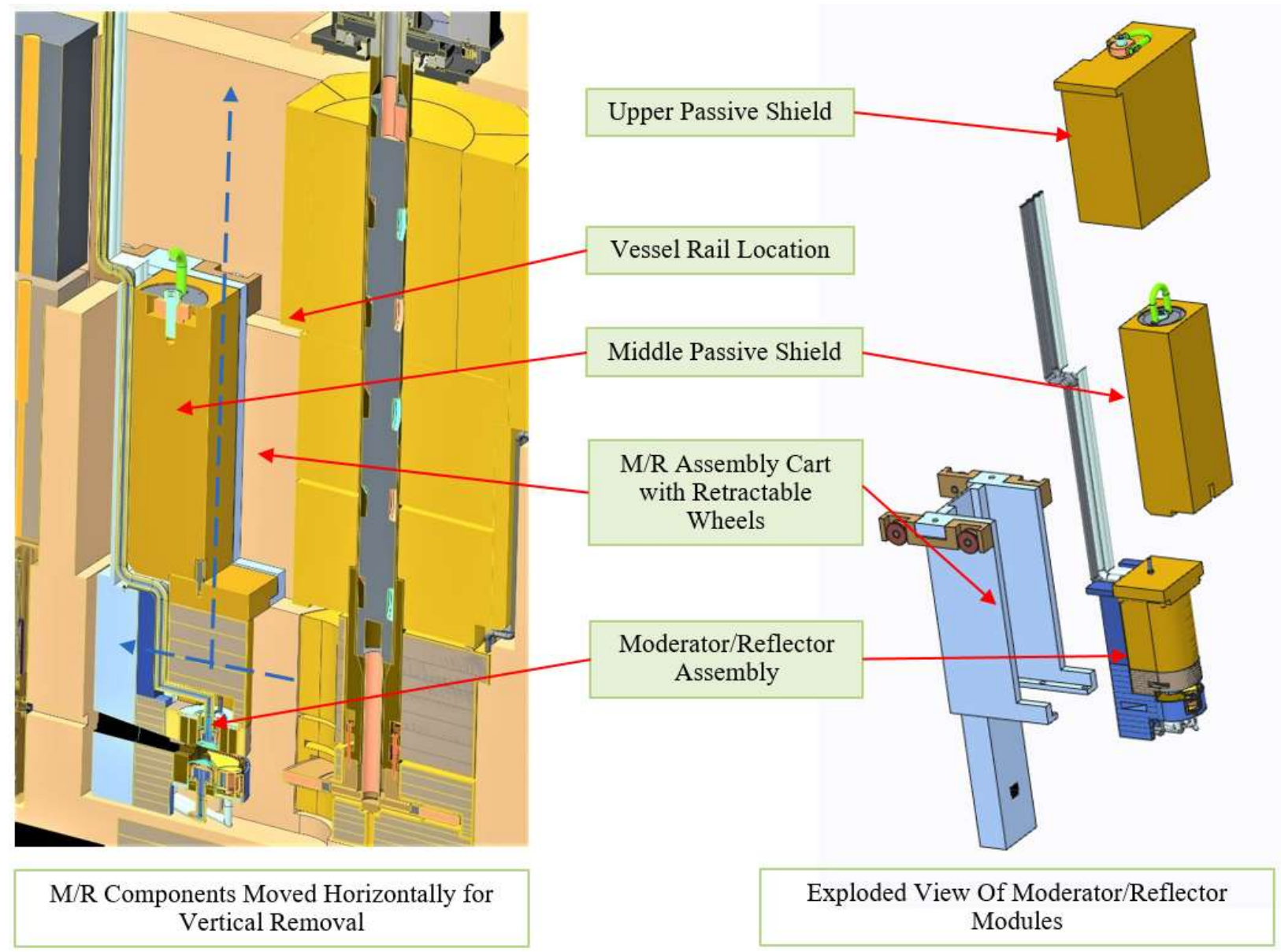

Figure 5.20. Vertical section view of target station core showing moderator-reflector assembly.

A preliminary Computational Fluid Dynamics (CFD) analysis of the bottom coupled moderator was performed to confirm that the cryogenic system is conservatively sized and that the flow inside the moderator container will have a uniform density. Two hydrogen flow rate cases were analyzed, both with pulsed (3000 J/cc-pulse in aluminum and $800 \mathrm{~J} / \mathrm{cc}$-pulse in Liquid Hydrogen), $15 \mathrm{~Hz}$ input heating as determined by the neutronics analysis. Figure 5.21 shows the results for a flow rate of $1.2 \mathrm{l} / \mathrm{s}$ or half the currently proposed STS cryogenic hydrogen flow rate. As indicated in the plot, the bulk temperature rise is approximately $2 \mathrm{~K}$, and the maximum temperature is $\sim 23 \mathrm{~K}$ in the recirculation zone. This analysis confirms that a compact neutron source does not exhibit unusual moderator heating. It also shows that as moderator designs evolve during the next phase of design, the currently oversized cryogenic system can be resized to provide performance with a minimal cost and configuration impact. 


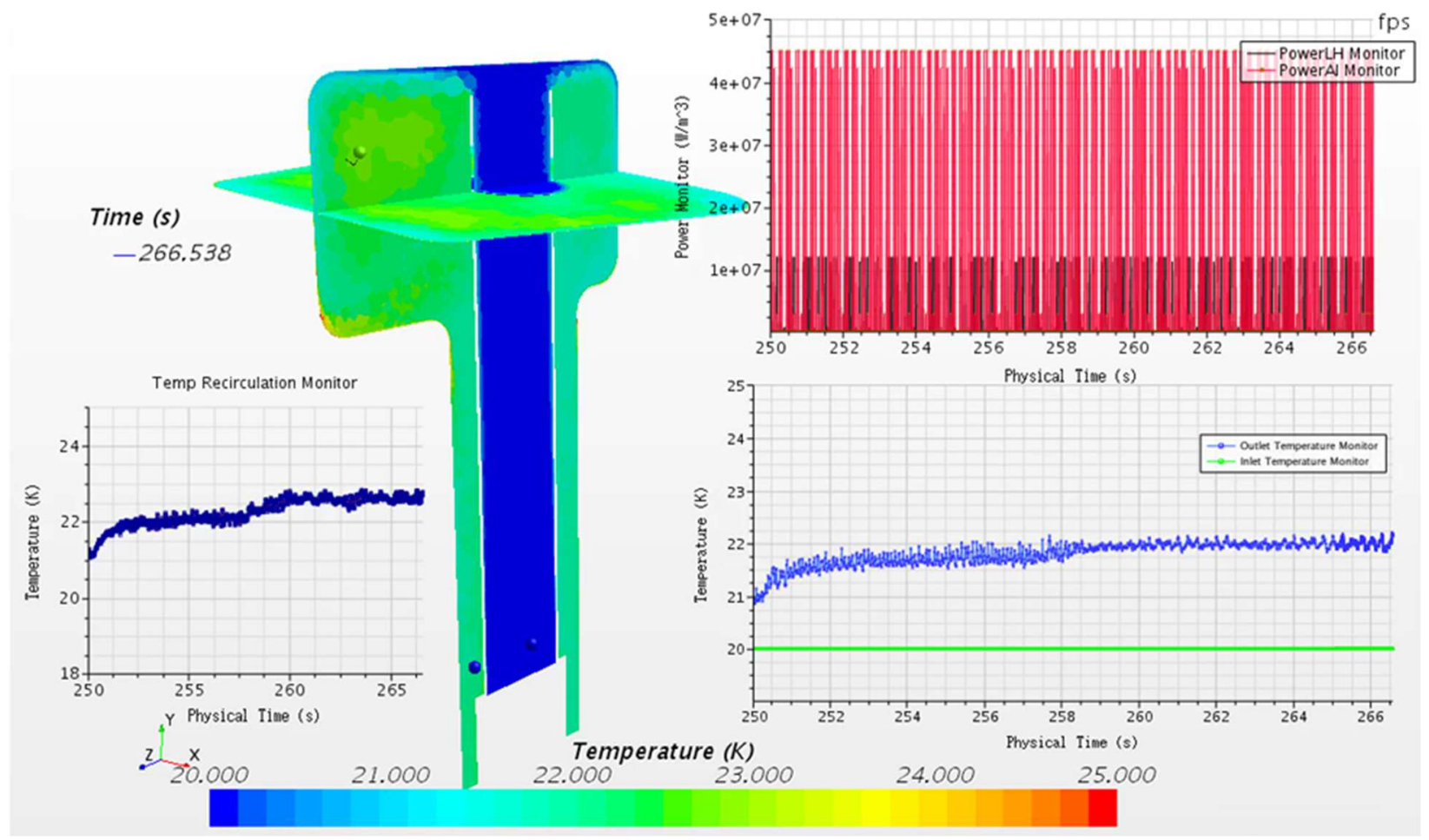

Figure 5.21. CFD predicted temperature distribution in the lower coupled moderator with a liquid hydrogen flow rate of $1.25 \mathrm{l} / \mathrm{s}$.

The CFD analysis was accomplished under the umbrella of a time scale study in the coupled bottom moderator. This time scale study established the time response of the system to a pulsed volumetric heat source regarding thermal-hydraulic performance. It was established that pulsed volumetric heat sources emulating energy deposition in matter due to the spallation process - would produce a detectable response in the moderator system in the order of milliseconds. Therefore, simulations can be run with pulses depositing energy in the moderator system for 1 millisecond duration instead of the 1 microsecond of the real beam pulse. The ultimate purpose of this approach was the efficient use of computational resources and optimization of the required time for completion of a single simulation run. All studied cases were performed under single phase, turbulent, unsteady, fully coupled (fluid-solid) conditions following standard CFD best practices guidelines for mesh and solution convergence.

Manufacturing and assembly of moderator reflector plugs has proved to be difficult due to a concentration of challenging issues, including aluminum welding, provisions for intense heat removal, cryogenic interfaces and packaging of beryllium. To address these issues, the STS moderator-reflector plug has been segmented into modules designed to be individually fabricated and tested prior to assembly. An exploded view of the plug with these modules separated is shown in Figure 5.22. Another advantage of modular design is the ability to adapt the assembly for to accommodate alternative technologies and different moderator sizes as the facility matures. 


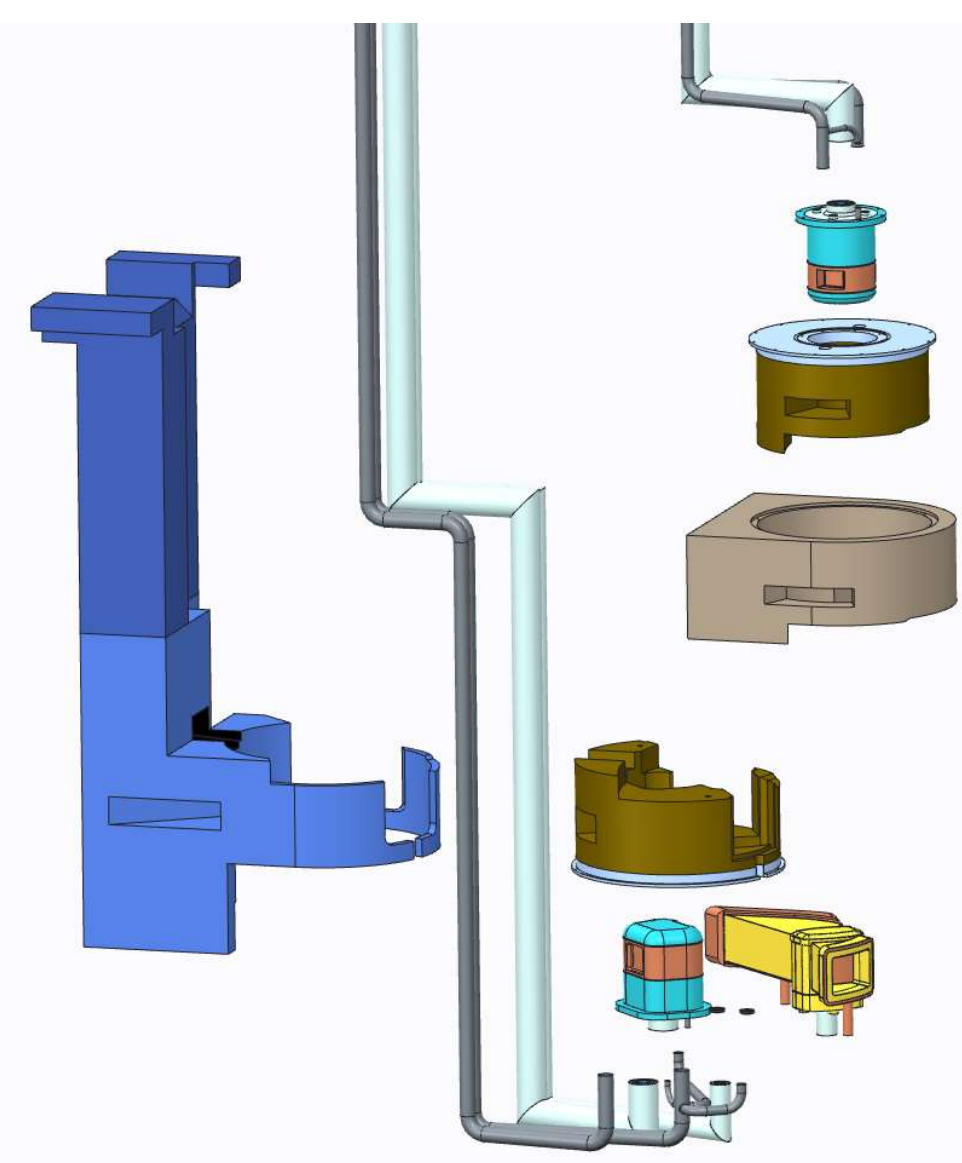

Figure 5.22. Exploded view of the moderator-reflector plug showing modular construction.

\subsubsection{Target}

The original neutronics studies were based on a stationary solid target able to handle the high proton beam flux density required for a compact source. Water cooled tungsten was selected early in the physics studies given the long successful history of this material in several facilities over several decades. Continuing to rely on this history, the original STS design included a fixed target like those used at ISIS and LANSCE. A design for $500 \mathrm{~kW}(10 \mathrm{~Hz})$ operation was developed which featured 17 tungsten/tantalum clad plates. The plate thickness varied to maintain a surface temperature of approximately $100^{\circ} \mathrm{C}$ facing $3 \mathrm{bar}, 10 \mathrm{~m} / \mathrm{s}$ water cooling. Figure 5.37 shows the peak tungsten temperature for the $2^{\text {nd }}$ plate with the highest heat generation and the $15^{\text {th }}$ plate which was much thicker to maintain the same surface temperature with a lower heat generation rate. The peak for these was approximately $175^{\circ} \mathrm{C}$ and $230^{\circ} \mathrm{C}$. For comparison, a rotating target response is shown, with a peak of only approximately $100^{\circ} \mathrm{C}$ with a period of approximately 3 seconds. Accident analysis of the stationary STS configuration indicated that residual heating would result in unacceptable operating and safety conditions with loss of cooling leading to tungsten temperatures above $800^{\circ} \mathrm{C}$ with tungsten vaporization and possible structural failures if pre-moderator cooling not maintained. Fortunately, a rotating target distributes activation over a large volume; thus, compared to the original stationary compact target, the rotating target residual heat at shutdown after 10 years of operation for the same power increases by a factor of only 1.7, while the area for heat transfer to the shielding for a loss of cooling accident increases by a factor of about 25 . This passively limits the peak temperatures due to decay heating of the assembly to around $350^{\circ} \mathrm{C}$ (Figure 5.23) as discussed in section 5.4.3.6. This is well below the $800^{\circ} \mathrm{C}$ temperature at which tungsten reacts with water vapor to create hydrogen gas and tungstic acid powder. Without the 
combination of an explosive gas and highly activated particulates the residual heat related accident scenarios can be safely resolved as described in Section 0 .

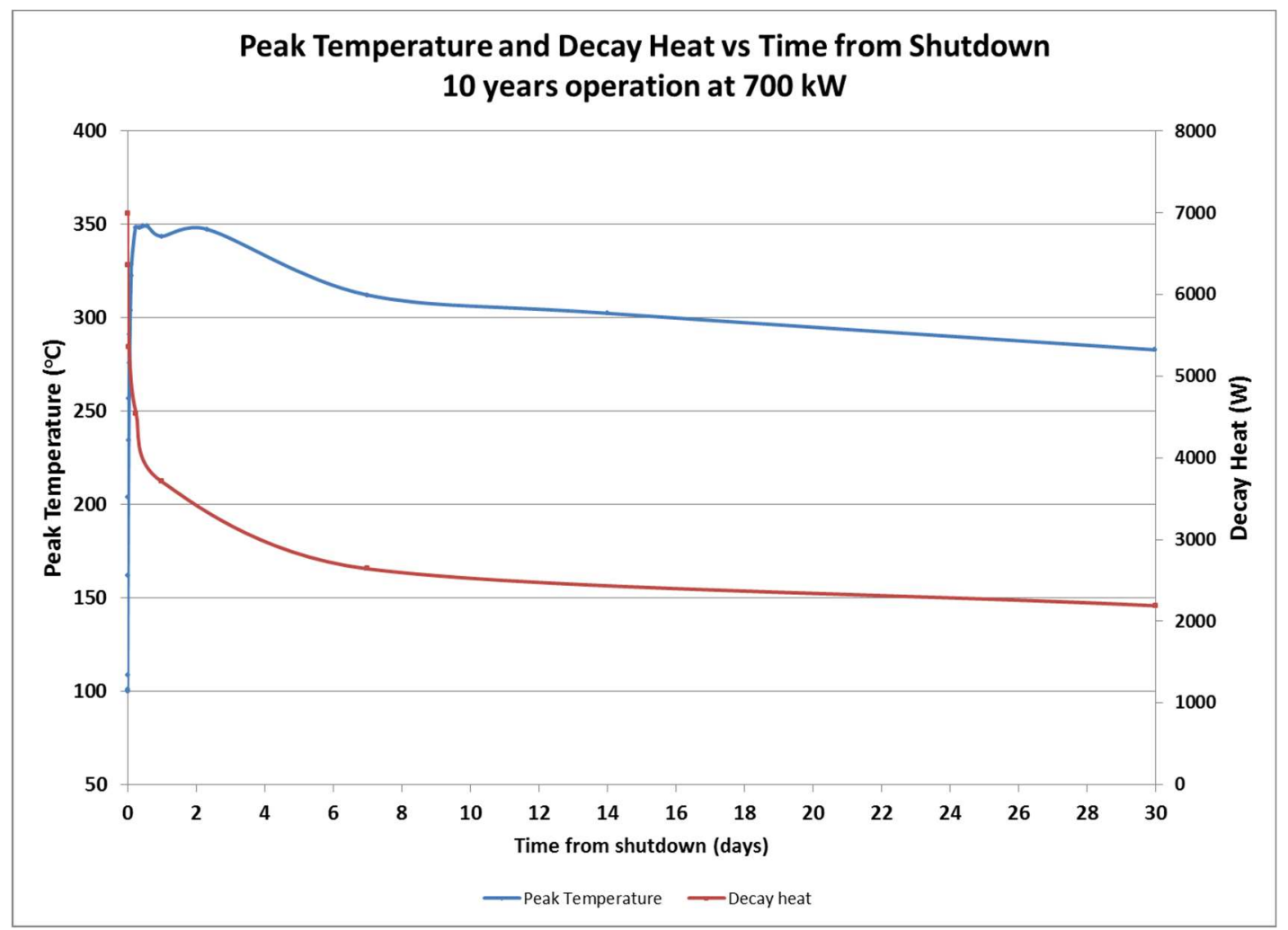

Figure 5.23. Maximum target temperature and decay heat versus time after a loss of coolant accident with beam trip by target protection system or seismic motions.

\subsubsection{Target description}

The decision to use a rotating target in STS was confirmed in October 2015 by an independent technical review committee [6]. The resulting target station configuration is shown in Figure 5.24. Positioning all active target components (e.g., motor, seals and sensors) in a low radiation area with hands-on maintenance capability is considered essential. Therefore, the proposed arrangement with the disk suspended on a vertical shaft supported by a drive assembly located outside the activation zone is the same as has been proposed at all facilities that have considered rotating targets [7][8]. 


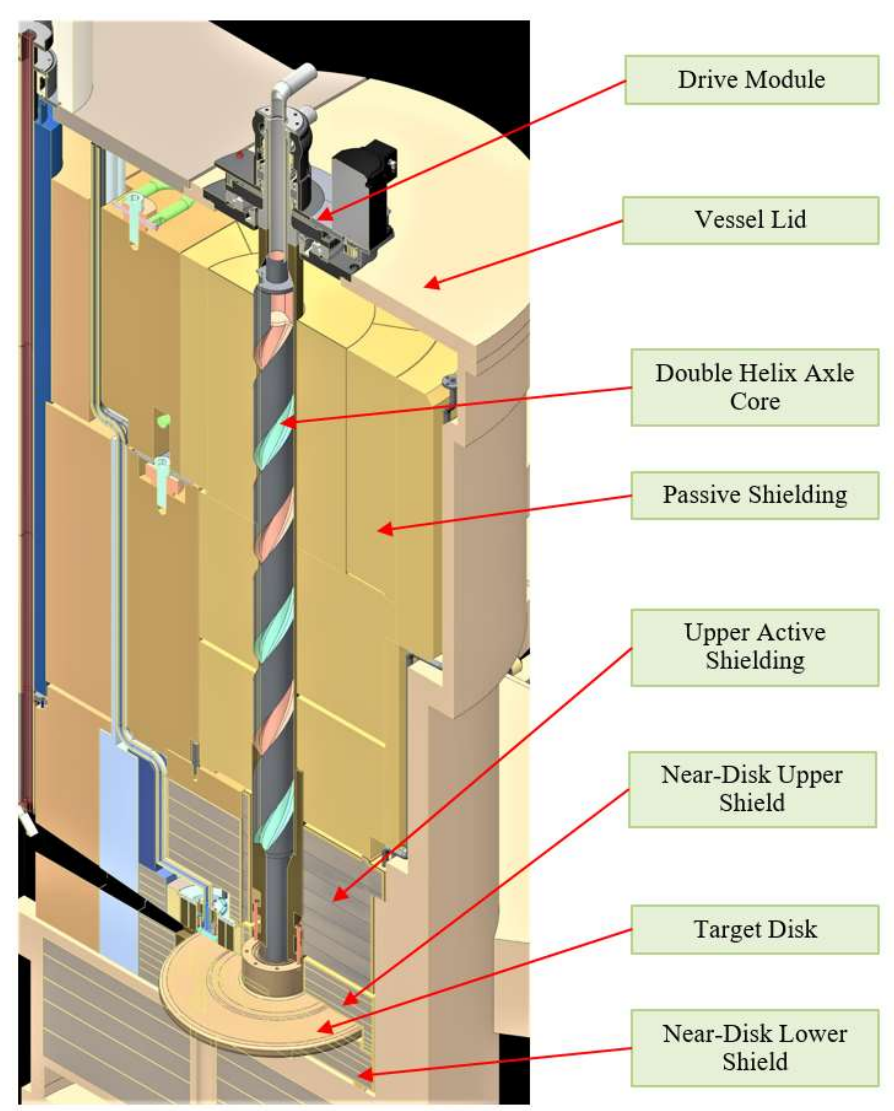

Figure 5.24. Vertical section view of target station core with rotating target assembly highlighted.

Table 5.2 summarizes many of the important target parameters. Significantly, the basic physical characteristics of the tungsten, such as height and depth, match the original stationary arrangement. Asynchronous operation will distribute the proton beam pulses uniformly over the full circumference of the disk and will simplify normal beam-on operations. The diameter of the disk was optimized to avoid interference with the forward beam lines and to make room for the moderators and reflector in front of the axle.

Table 5.2. General target design parameters

\begin{tabular}{lc}
\hline \multicolumn{1}{c}{ Parameter } & Value \\
\hline Target diameter & $1.14 \mathrm{~m}$ \\
Tantalum clad thickness & $1 \mathrm{~mm}$ \\
Tungsten/Ta plate thickness & $5 \mathrm{~cm}$ \\
Plate radial depth & $25 \mathrm{~cm}$ \\
Beam spot area (90\%) & $7.5 \mathrm{~cm} \mathrm{x} 4 \mathrm{~cm}$ \\
Beam frequency & $15 \mathrm{~Hz}$ \\
Beam Power & $700 \mathrm{~kW}$ \\
Rotation speed & $20 \mathrm{RPM}$ \\
Timing & Asynchronous \\
Shell material & $316 \mathrm{~L}$ \\
Coolant & $\mathrm{H}_{2} \mathrm{O}$ \\
\hline
\end{tabular}


Four subassemblies make up the drive assembly. A conventional 2-3 hp servo drive with an integral gear reducer will power a ring and pinon gear interface to the axle. This arrangement has proven to be very reliable and will simplify the independent replaceability of the drive. A single, commercial, graphite-onstellite, all-metal face seal will contain the cooling water in the target axle assembly. The proposed seal is rated for $0.83 \mathrm{MPa}$, well above the dead-head pump pressure of $0.35 \mathrm{MPa}$. The seal assembly is mounted on top of the drive assembly for independent replacement. A second seal is eliminated by separating the supply and discharge flows with a small gap with an acceptable by-pass flow rate; currently estimated to be less than $5 \%$ of the total. A large diameter $(\sim 0.75 \mathrm{~m})$ "slewing" bearing in combination with a radial bearing set provides the rigidity necessary for the suspended disk. A set of sensors will monitor inlet and discharge water temperatures, bearing vibration, coolant flow, leak detection and rotation speed. Each of the sensors will be mounted for independent replacement.

The 4.5-meter-long axle will be a machined unit that will precisely align the target disk to the drive bearings. It is currently envisioned to be a welded assembly with transition pieces on either end of a heavy walled cylinder enclosing a double helix, water-routing insert. The axle will bolt to the target disk with long, pre-tensioned stainless steel bolts or studs configured to provide access for a remotely operable cut-off tool. This will enable the separation of the axle from a spent disk as required for packaging. The target-axle joint will be sealed with two concentric metal O-rings with the possible addition of a center leak detection tap to remotely monitor for intraseal leaks. The axle assembly weighs $1710 \mathrm{~kg}$.

Figure 5.25 and Figure 5.26 show key details of the target disk. A total of sixteen, 5-cm-high tantalumclad, tungsten blocks 25 centimeters deep are arrayed around a 316L center hub and enclosed with a 316L shroud. The target blocks will have a uniform 1-mm-thick cladding to prevent water erosion of the tungsten. The configuration with two plate shapes was selected to allow the portion of the shroud covering the disks to be fabricated separately and the plates installed from the inner radius. The shroud will have block-separating features, flow-channeling vanes and stand-offs to ensure uniform, predictable flow. Approximately $12.6 \mathrm{l} / \mathrm{s}$ of water with a bulk temperature rise of $9 \mathrm{C}$ flows radially out over the top of the plates, between the front stainless steel window and the plate outer radius, and return on the bottom between the shell and plates with a variable flow gap of $1 \mathrm{~mm}$ to $2 \mathrm{~mm}$ designed to maintain constant flow velocity. The resulting water velocity of roughly $3 \mathrm{~m} / \mathrm{s}$ provides a convective heat transfer coefficient on the surface of the plates in the range of $1 \times 10^{4} \mathrm{~W} / \mathrm{m}^{2}-\mathrm{K}$ to $2 \times 10^{4} \mathrm{~W} / \mathrm{m}^{2}-\mathrm{K}$. The velocity margin provides an allowance in the flow gaps for pressure induced expansion of the shroud and for target block and shroud fabrication tolerances. The hub includes the axle joint and two rows of 32 radial water distribution holes each for supply and return flow. As currently conceived the target blocks are not connected nor spring loaded. Note that while the target has an upset retainer pin under the disk, there are no bearings inside the monolith shield. The disk assembly weighs $1010 \mathrm{~kg}$, including $621 \mathrm{~kg}$ of tungsten and $34 \mathrm{~kg}$ of tantalum. 


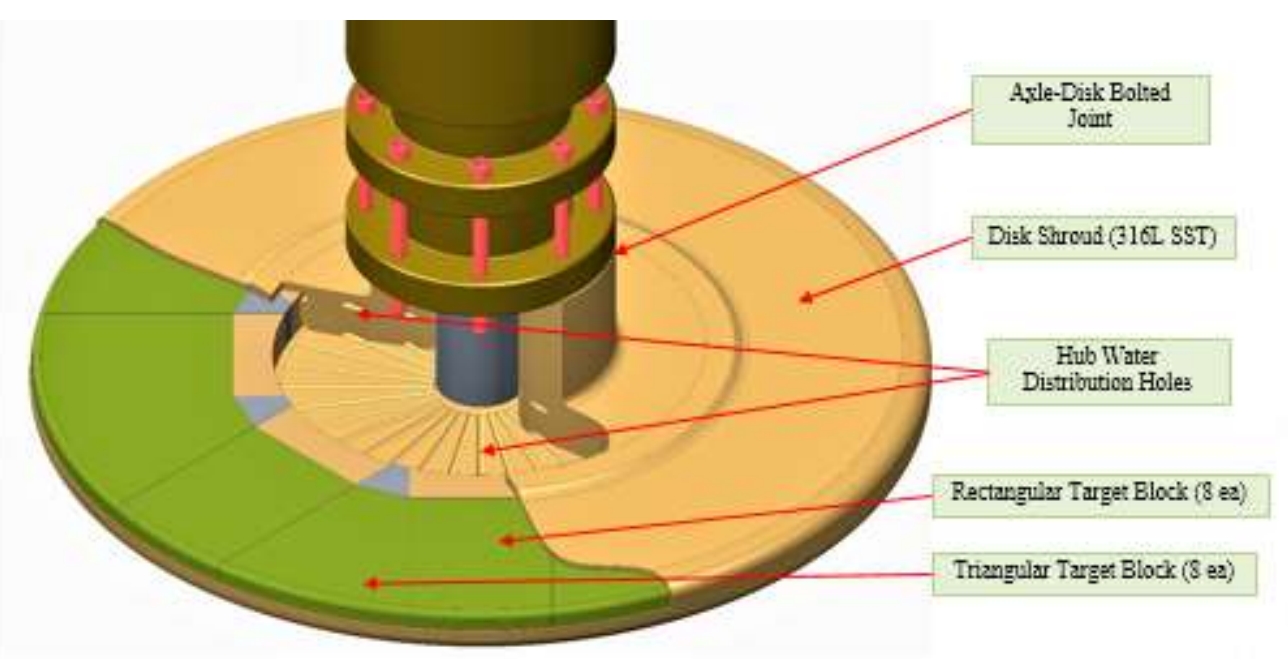

Figure 5.25. Sectioned target disk showing internal details.

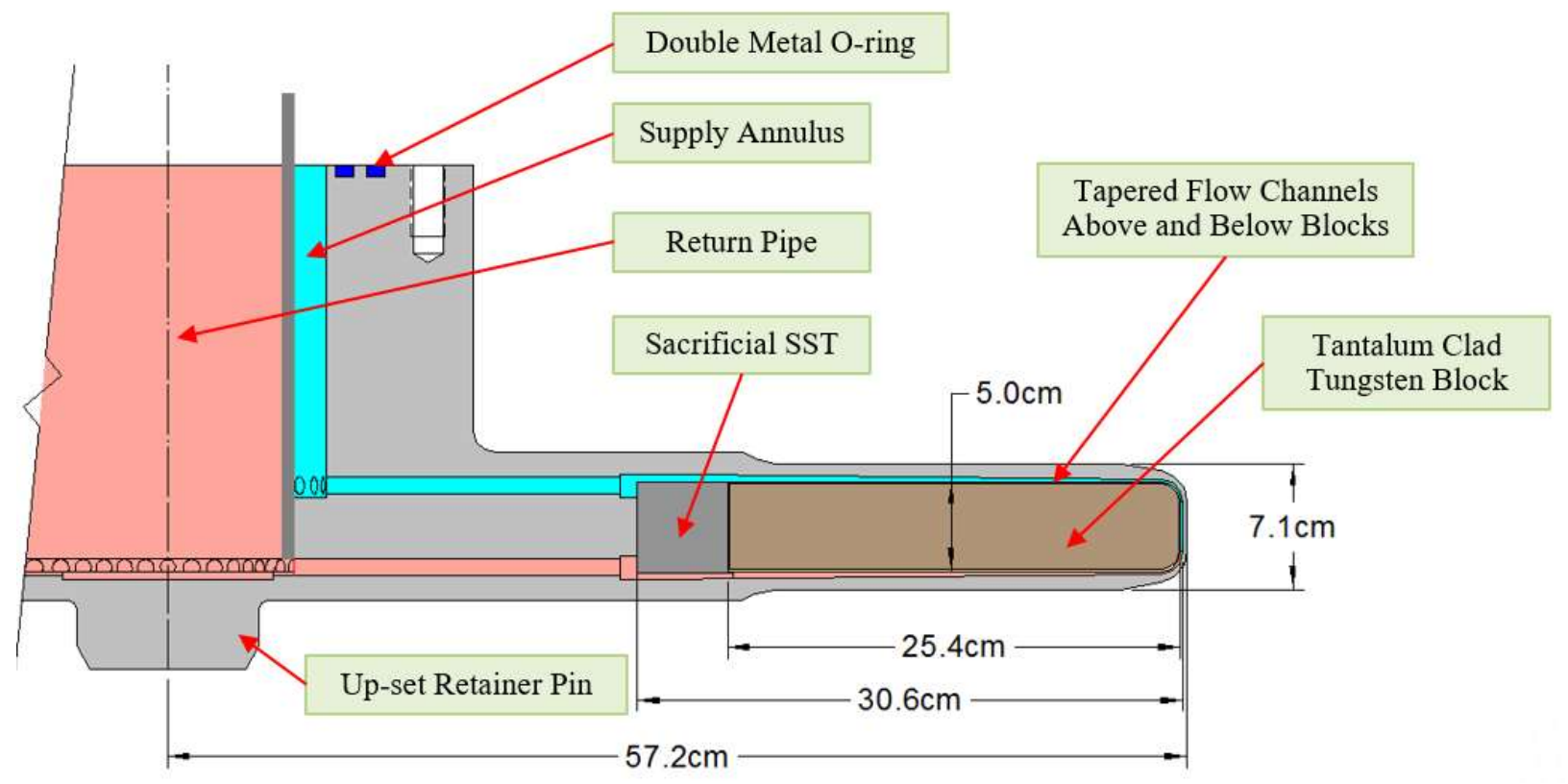

Figure 5.26. Target disk section view.

\subsubsection{Target analysis}

Energy deposition data calculated in the neutronics study as described in Section 5.1.4 was imported into an ANSYS 18.0 model used to analyze the thermal and structural characteristics of the proposed design for $15 \mathrm{~Hz}$ or $700 \mathrm{~kW}$ operation. The peak energy deposition per pulse was $8.15 \times 10^{7} \mathrm{~J} / \mathrm{m}^{3}$ in the tungsten. The adiabatic temperature rise in tungsten for this would be $31^{\circ} \mathrm{C}$. Figure 5.27 shows the computed midplane tungsten temperatures for a full revolution for the first 45 pulses with $30^{\circ} \mathrm{C}$ water cooling and a heat transfer coefficient of $2 \times 10^{4} \mathrm{~W} / \mathrm{m}^{2}-\mathrm{K}$ on all outer surfaces. Peak values were approximately $60^{\circ} \mathrm{C}$, consistent with the adiabatic rise estimate. 


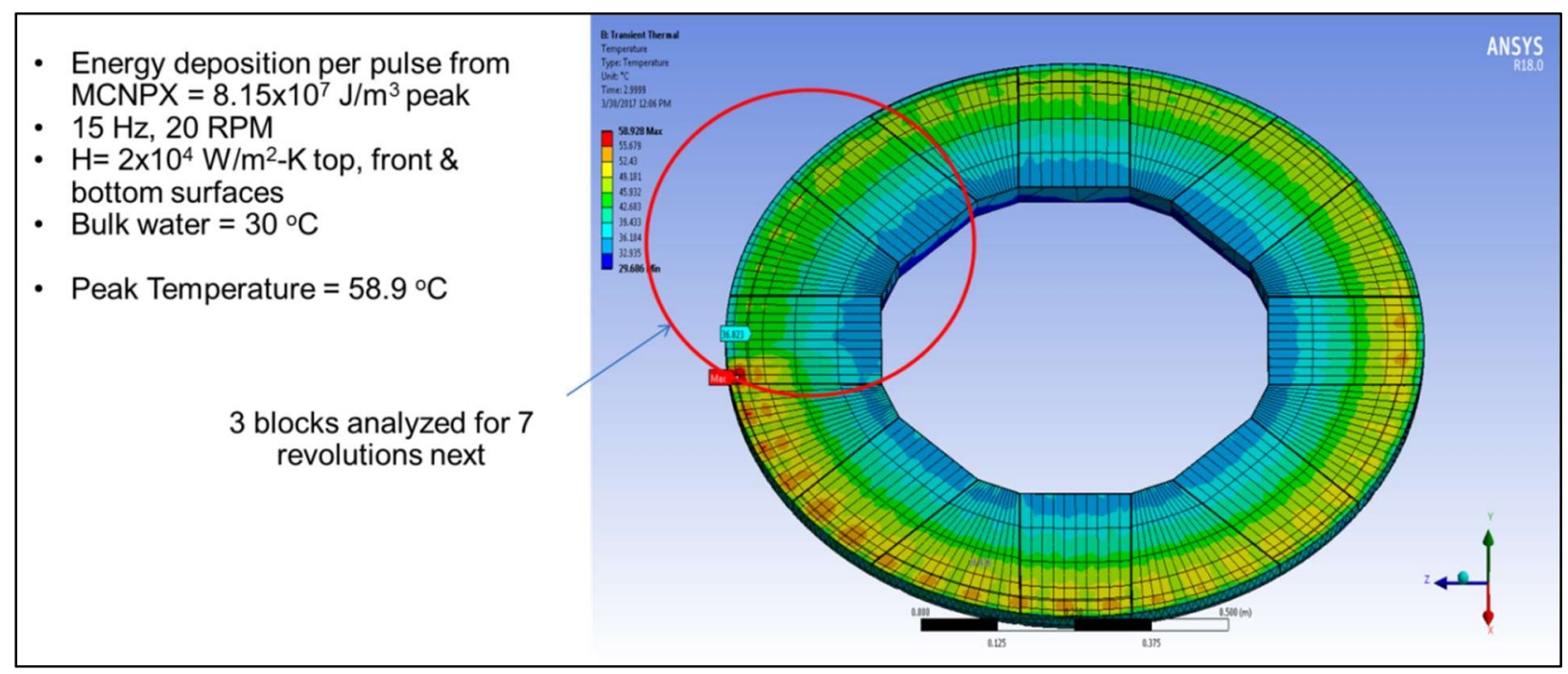

Figure 5.27. Midplane temperatures over first revolution- peaks $\sim 60^{\circ} \mathrm{C}$.

Having established the full cycle target performance, a simplified model with three representative blocks was used to analyze the target blocks in detail. It was determined that seven revolutions, $21 \mathrm{sec}$, are required for the target blocks to nearly reach the maximum steady-state thermal cycle as shown in Figure 5.28. The temperatures after 4 pulses into the $7^{\text {th }}$ revolution are shown in Figure 5.29. At the locations for peak energy deposition in the mid-plane, the temperatures cycle between approximately $70^{\circ} \mathrm{C}$ just before a pulse and $95^{\circ} \mathrm{C}$ just after a pulse. The peak water cooled surface temperatures are approximately $60^{\circ} \mathrm{C}$, giving a large margin to boiling. 


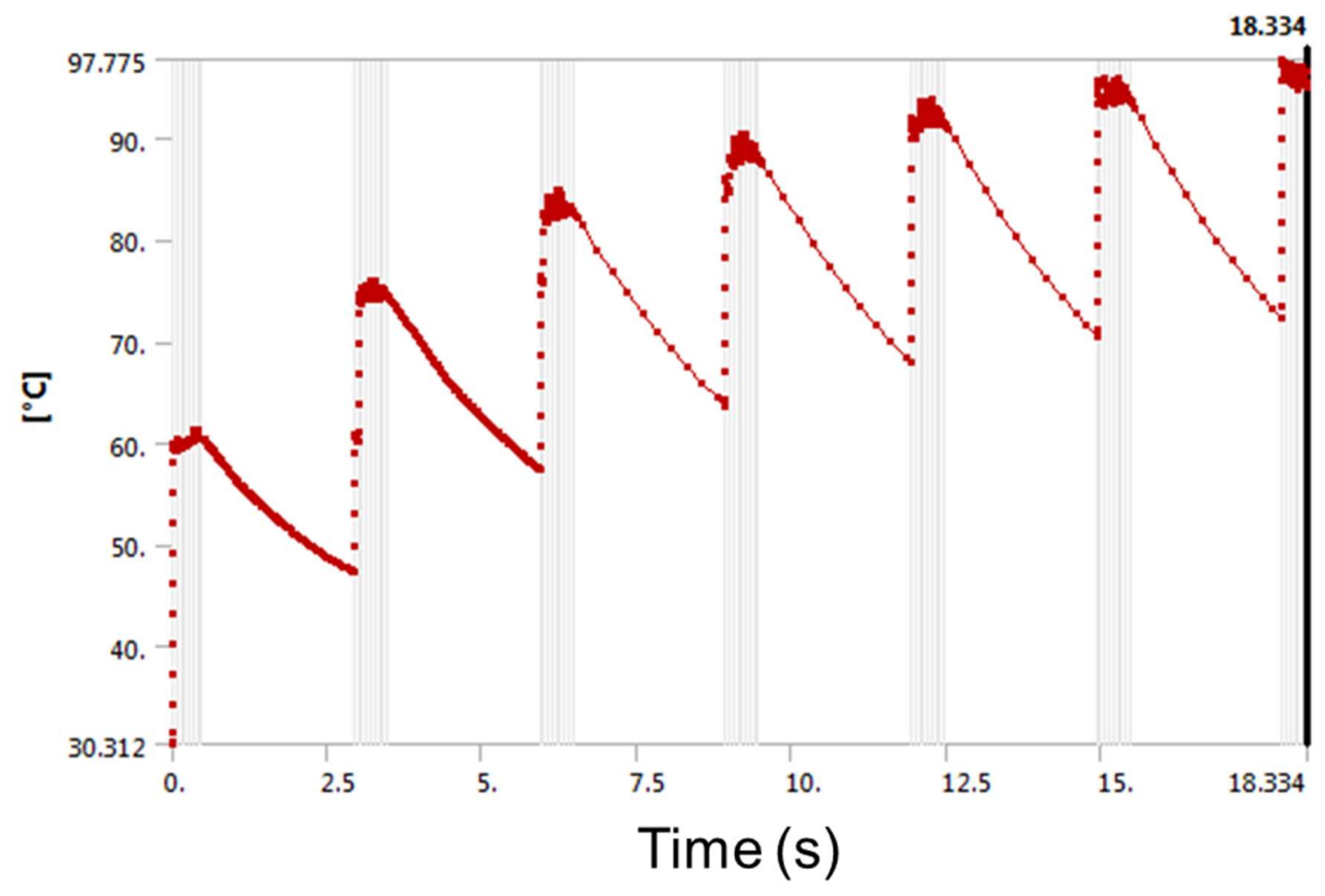

Figure 5.28. 3 Block model global peak temperature versus time for first 6 revolutions and 4 pulses into $7^{\text {th }}$ revolution.

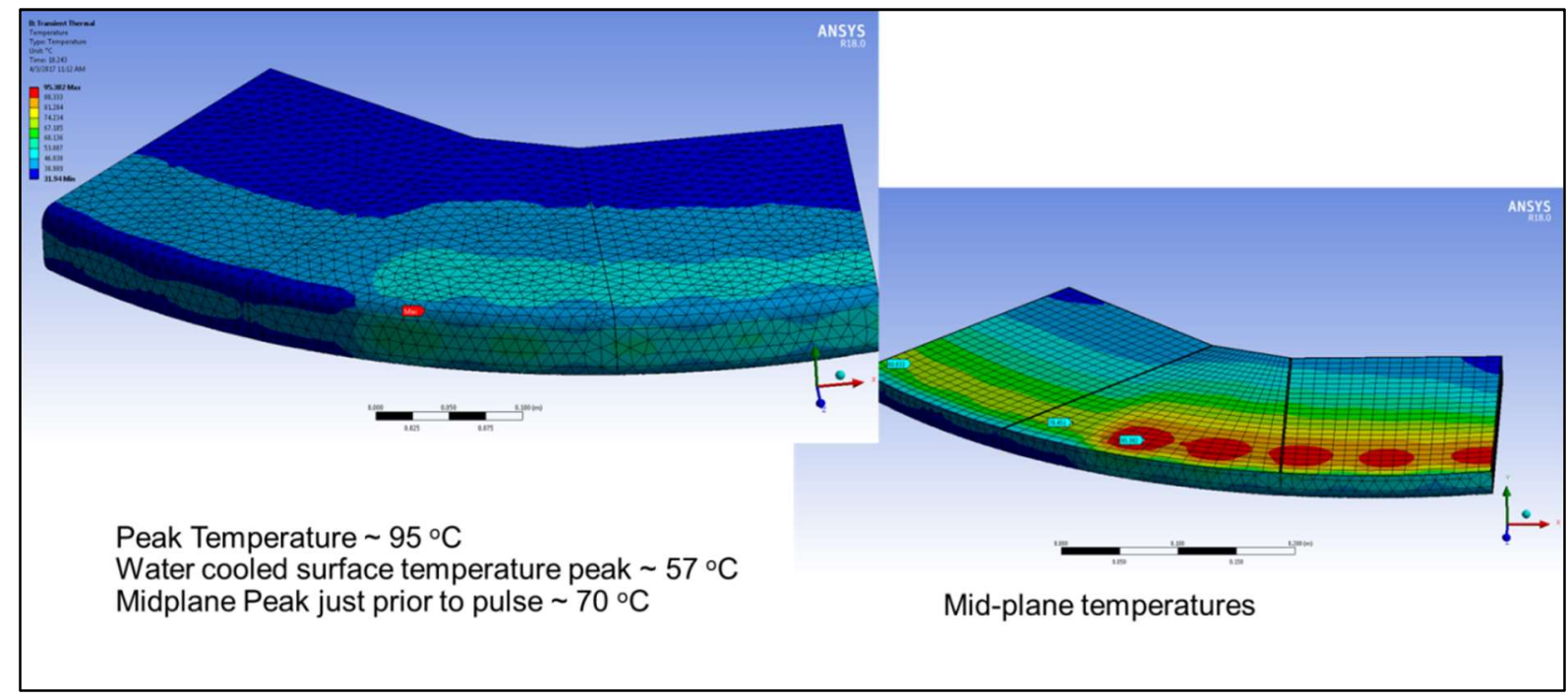

Figure 5.29. Temperatures after 6 revolutions plus 4 pulses into $7^{\text {th }}$ revolution.

Figure 5.30 and Figure 5.31 show the tantalum and tungsten surface Von Mises stresses after seven revolutions due to thermal stress. This does not include residual stress from fabrication or thermal shock. Figure 5.32 shows the midplane Von Mises stress levels. The peak is $61.7 \mathrm{MPa}$ and occurs on the outer surface of the tungsten blocks. This is well below the assumed limit of $200 \mathrm{MPa}$. 


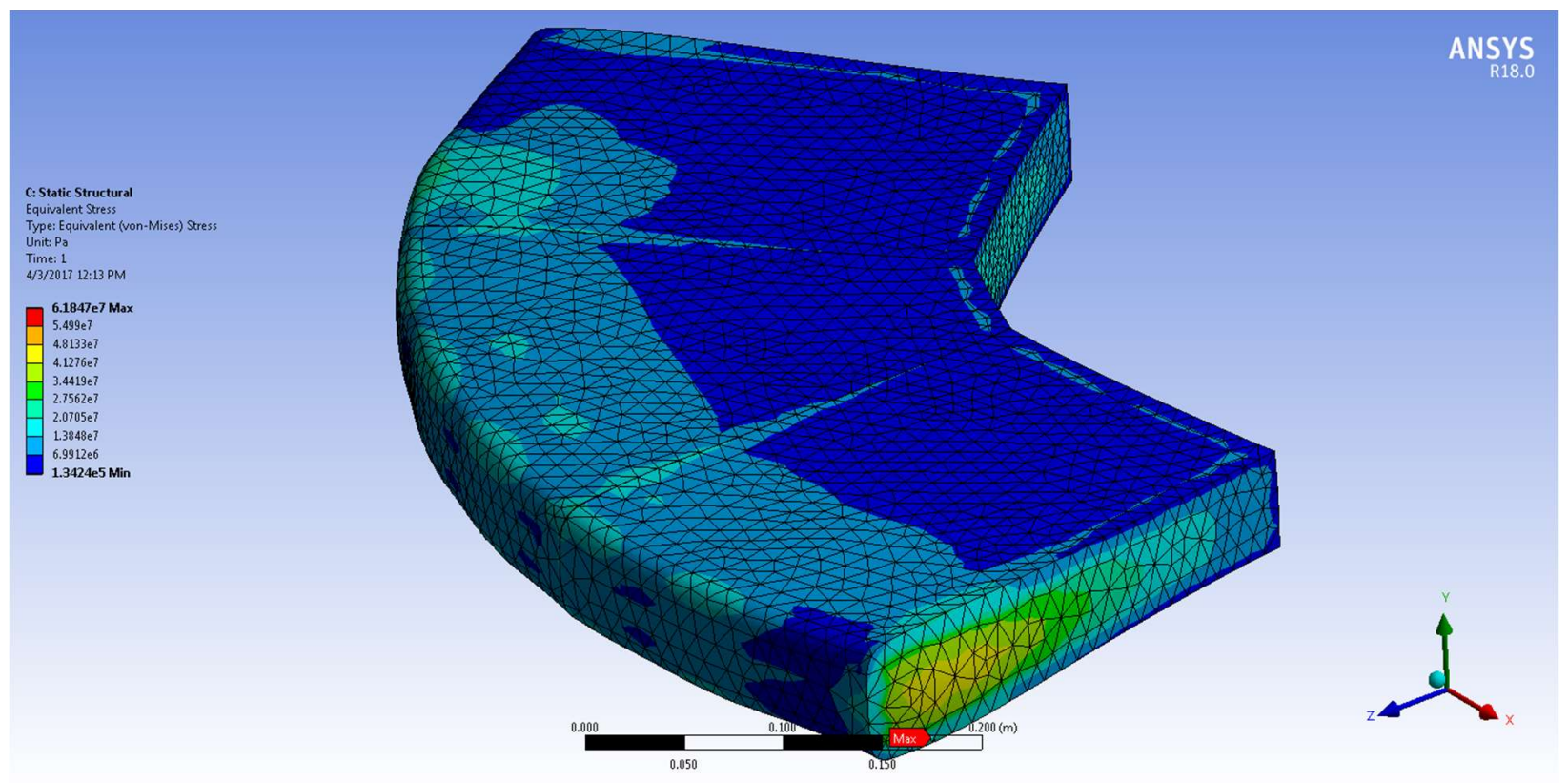

Figure 5.30. Tantalum surface Von Mises Stress - Peak 63 MPa.

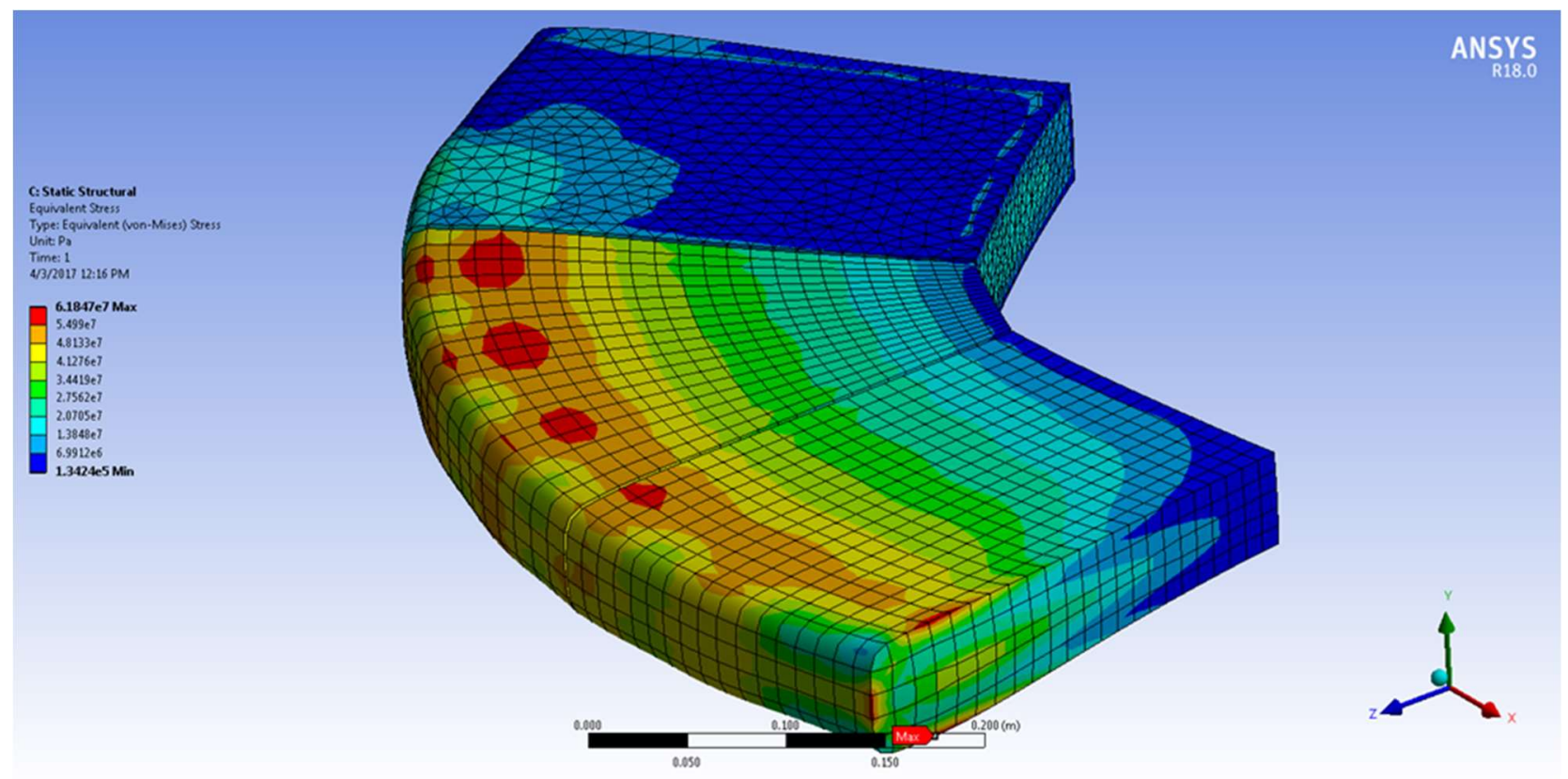

Figure 5.31. Tungsten surface Von Mises stress with clad hidden on two blocks. 


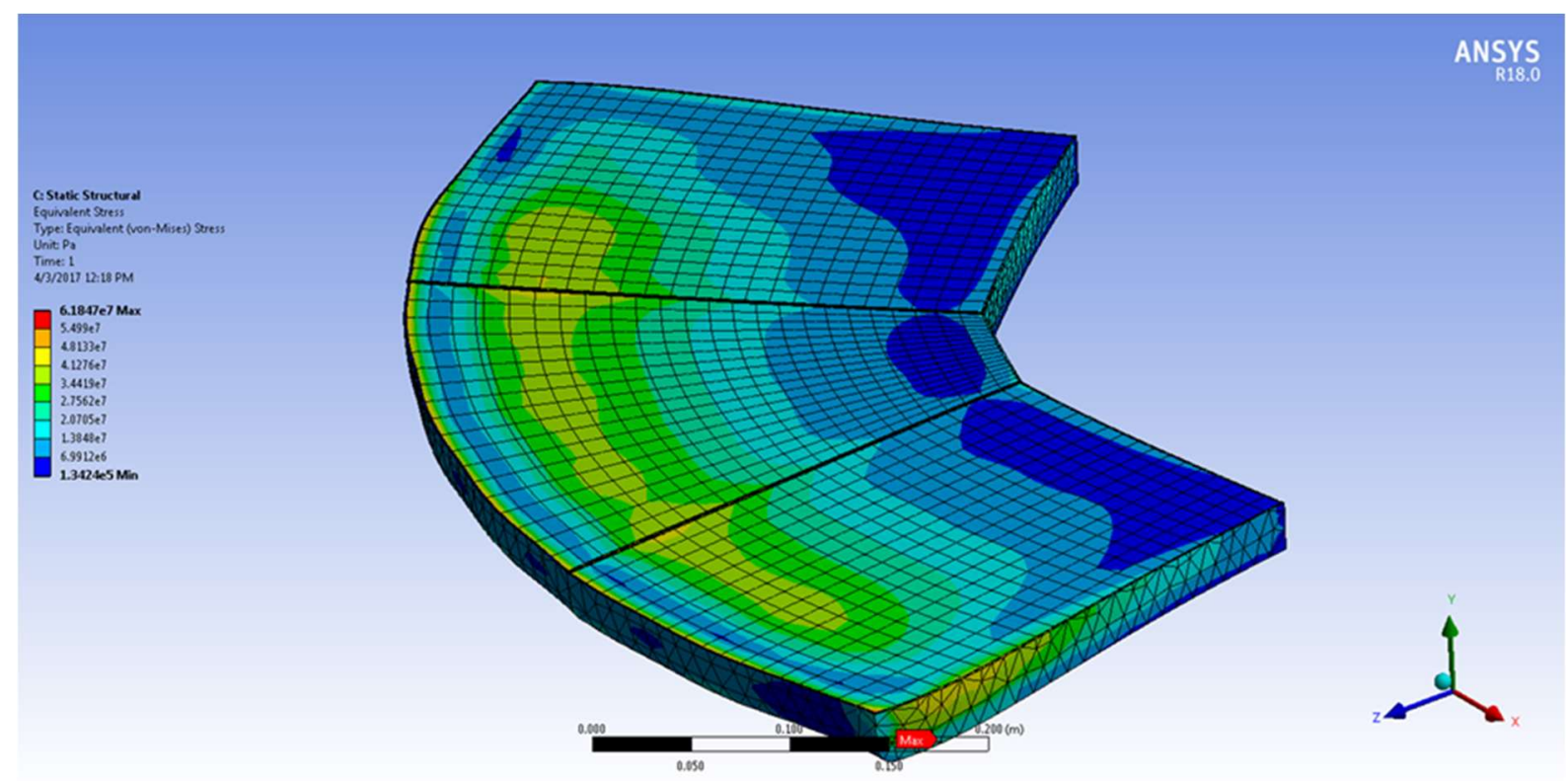

Figure 5.32. Mid-Plane Von Mises Stress.

Maximum $1^{\text {st }}$ Principal stress is shown in Figure 5.33 and Figure 5.34 since tungsten may have low ductility after irradiation and the failure mode may be due to peak tensile stresses. As expected, the tungsten stresses are compressive on the midplane and tensile on the surface. The peak tensile stress is 68.6 $\mathrm{MPa}$, and at this location the range is from approximately $10 \mathrm{MPa}$ just before the pulse to the 68.6 $\mathrm{MPa}$ peak. Figure 5.35 shows the thermal expansion of the target blocks after seven revolutions assuming constraint at the hub interface.

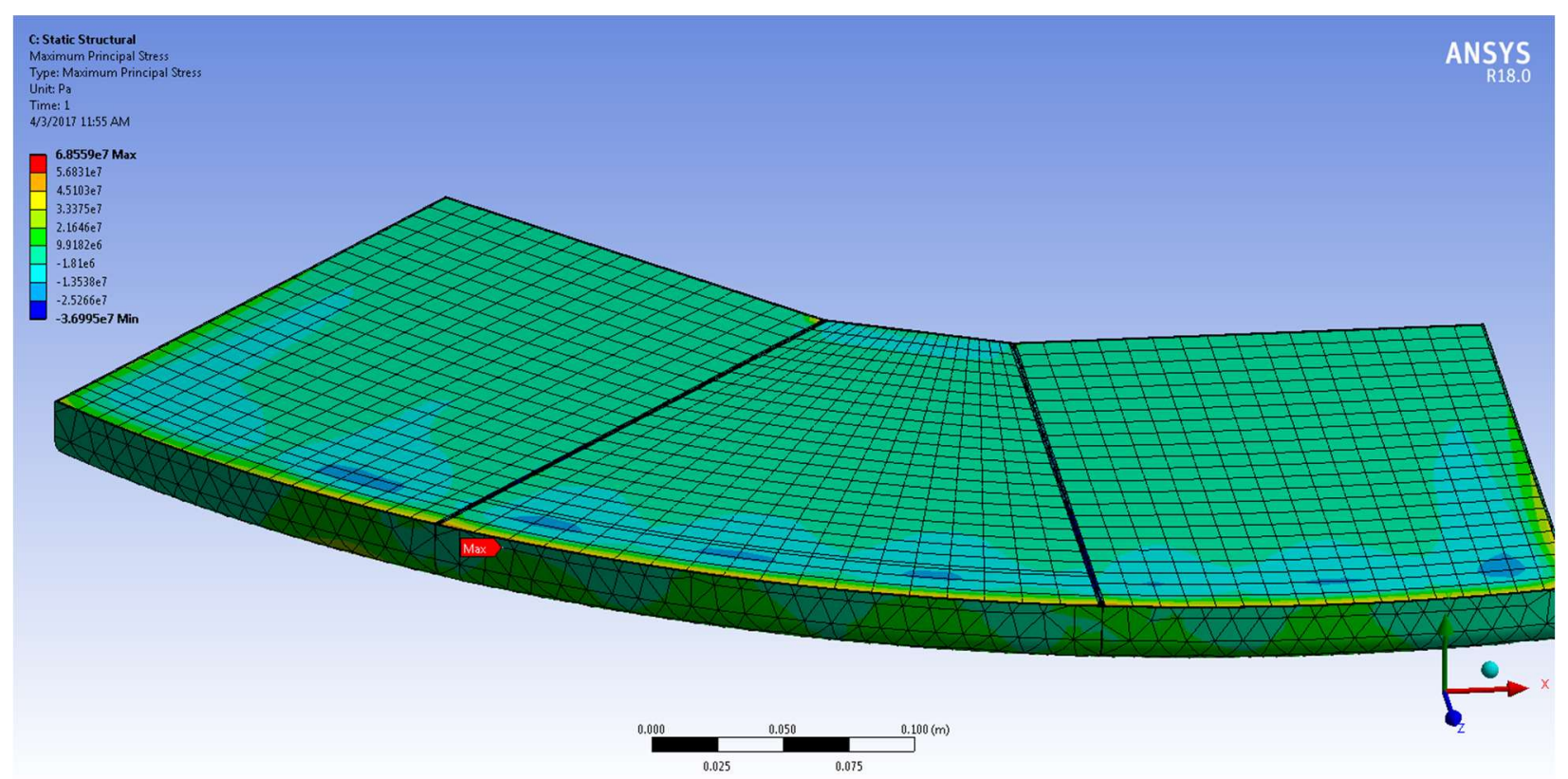

Figure 5.33. Maximum Principal Stress. 


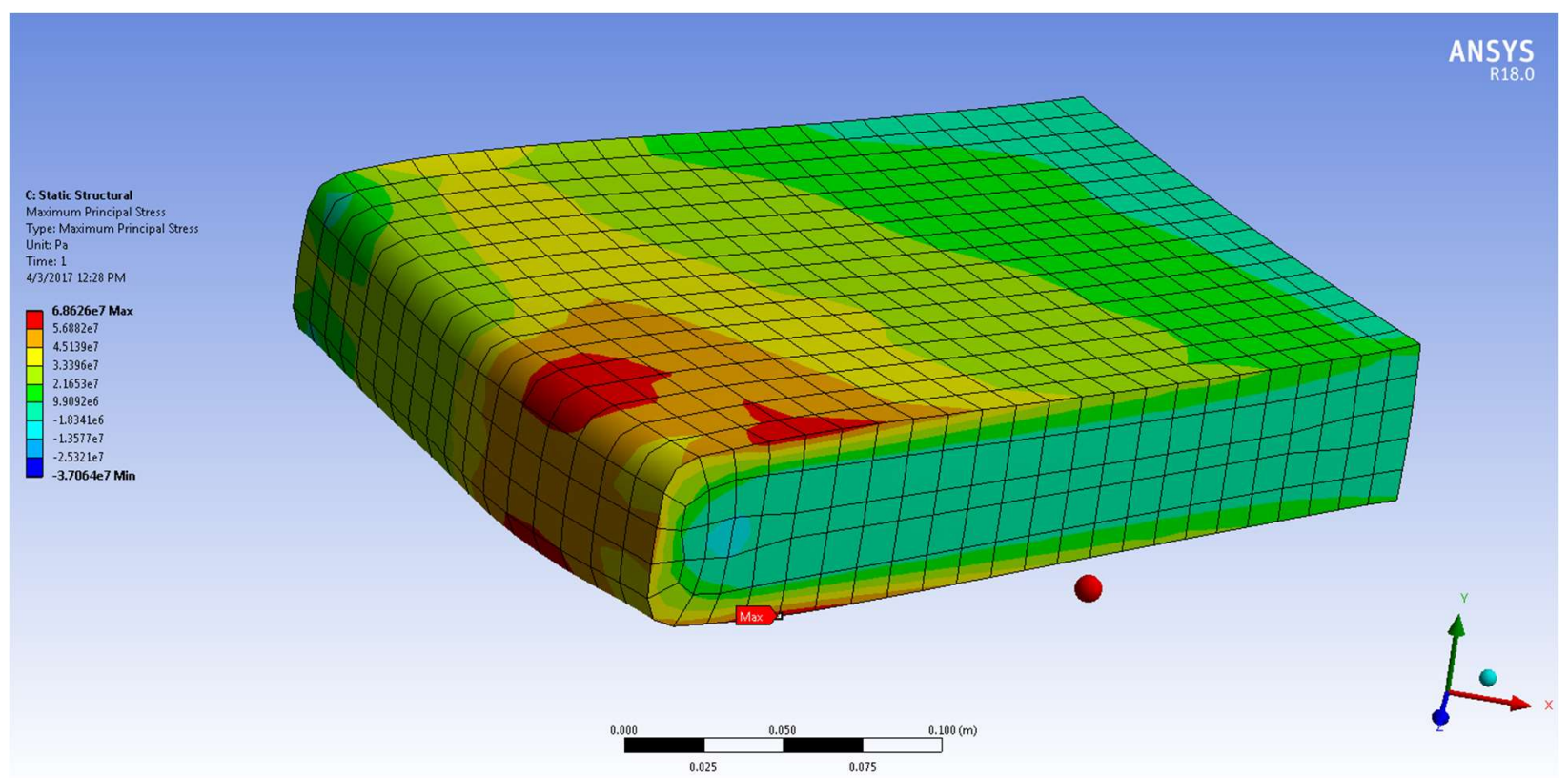

Figure 5.34. Maximum Principal Stress on Tungsten Block 1 - 68.6 MPa.

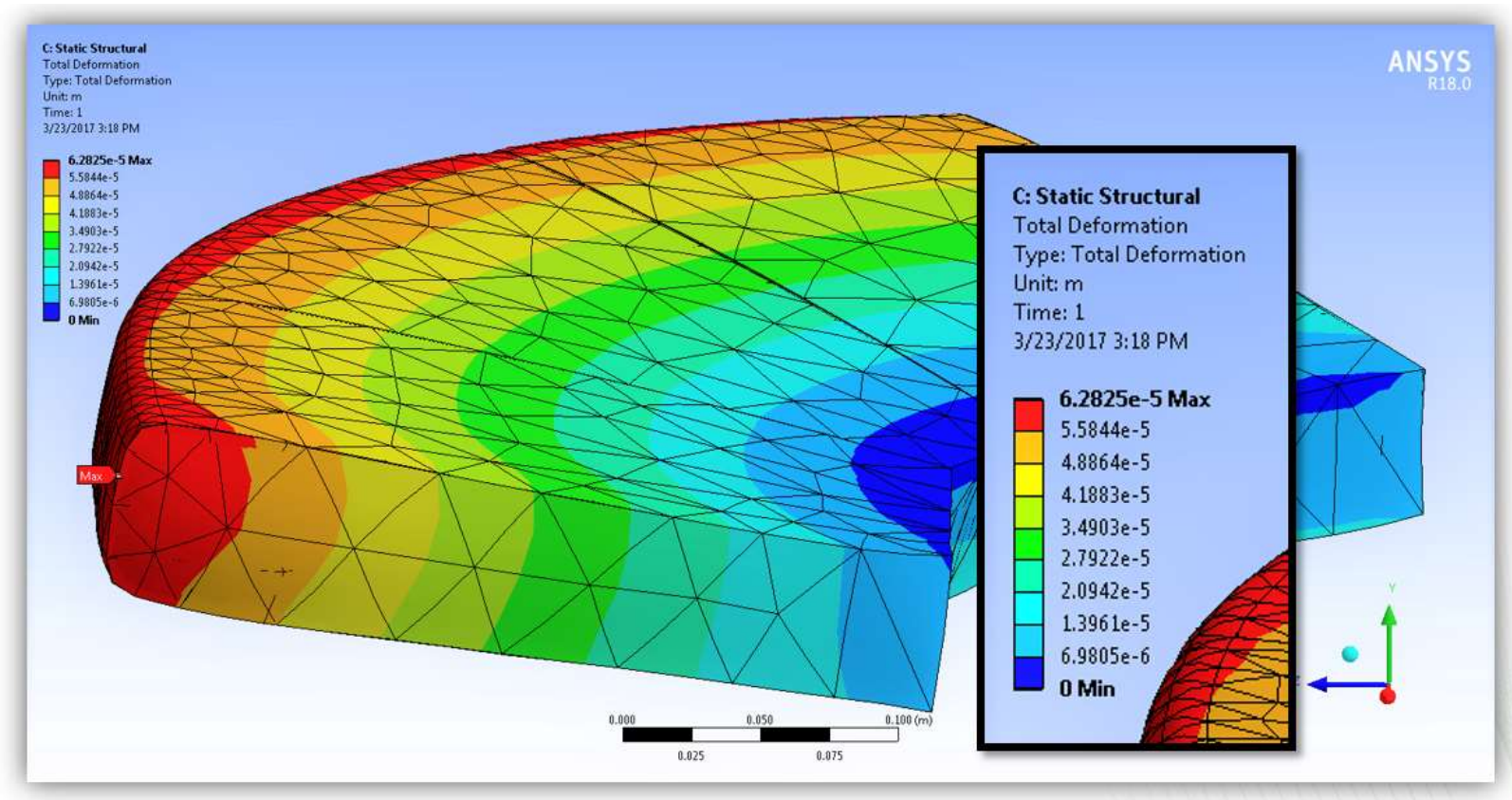

Figure 5.35. Tungsten plate thermal expansion after seven revolutions.

To achieve maximum neutron production, the target shroud is designed to accommodate full length (25 $\mathrm{cm})$ target blocks. It will be fabricated from 316L stainless steel (SA-240) in accordance with the fabrication standards of the ASME pressure vessel code. The Code properties for 316L plate are provided in Section II Part D: Table 5A, "Maximum Allowable Stress Values S for Ferrous Materials." For temperatures, up to $150^{\circ} \mathrm{C}$, the maximum allowable stress is set at $115 \mathrm{MPa}$.

To keep the shroud wall thickness as thin as possible, the process water system will be designed to limit the maximum internal pressure to less than 0.35 MPa. As shown in an ANSYS analysis of the target 
shroud (Figure 5.36), the maximum point stress at this pressure is approximately $164 \mathrm{MPa}$ with a maximum deflection of $0.3 \mathrm{~mm}$. Maintaining a low pressure will require the use of relatively larger pipes and connections to limit flow related pressure drops. As noted earlier, the increased gap between the shroud and the target blocks due to the pressure induced deflection will be considered in the thermalhydraulic design of the coolant flow channels.
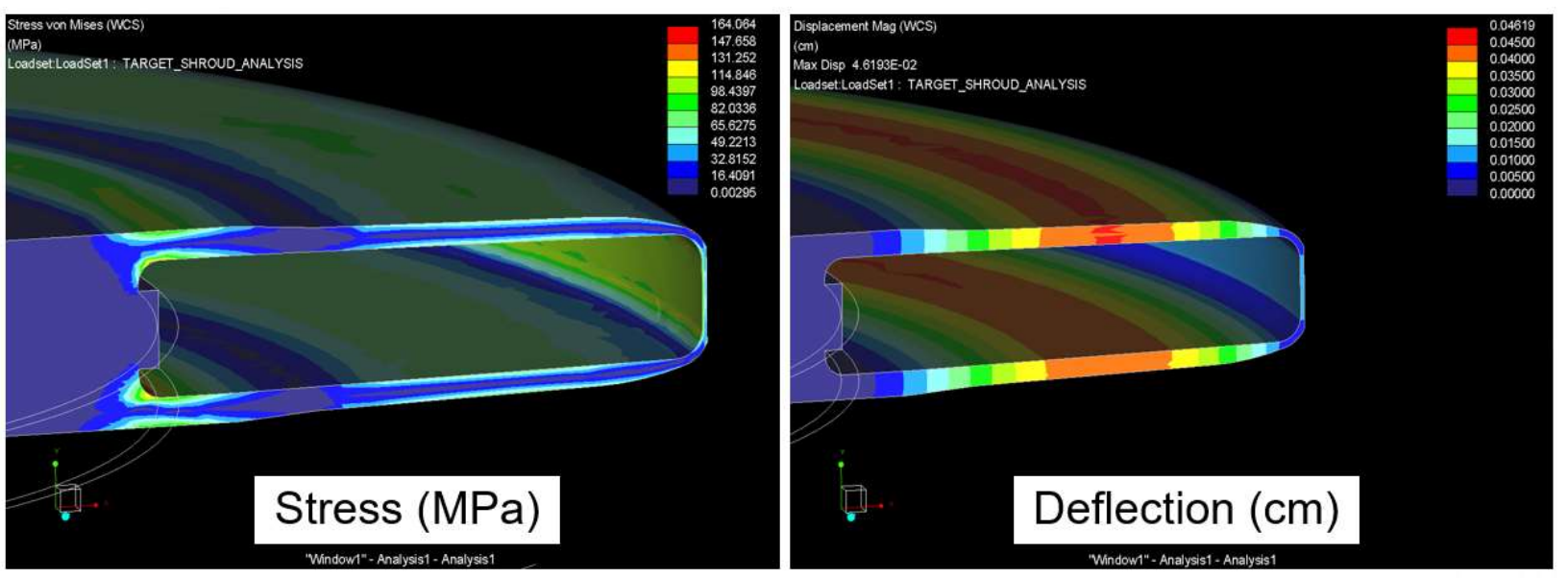

Figure 5.36. Calculated operating stress and deflection plots with $0.35 \mathrm{MPa}$ internal water pressure.

While the basic thermal stress analysis indicates the target blocks are within the operating range expected for a long-lived system, several other stress factors will be considered in the later stages of design. For example, the HIP fabrication process bonds the tantalum to the tungsten at a relatively high temperature. This results in a residual stress at the bonding interface as the assembly is cooled. Quantifying this stress and understanding operating conditions that might relieve it is difficult but and effort will be made to relate the STS values with those in successfully operating targets. In addition, pulse induce shock waves are known to occur inside the tungsten blocks. As with the bonding stress an effort will be made to understand how this stress will impact the life of the target blocks, especially with respect to the relatively large size of the proposed STS blocks.

A comparison of the STS target operating parameters with other facilities is provided in Table 5.3. Note that in almost all cases the STS blocks are more lightly loaded than the stationary targets. Of particular importance is the substantially lower pulse rate.

Table 5.3. Summary of various tantalum clad tungsten target thermos-mechanical factors

\begin{tabular}{|c|c|c|c|c|c|c|}
\hline Facility & KENS & ISIS TS-1 [9] & $\begin{array}{c}\text { ISIS TS-2 } \\
{[9]}\end{array}$ & LANSCE & $\begin{array}{c}\text { CSNS } \\
\text { (unconfirmed) }\end{array}$ & STS \\
\hline $\begin{array}{c}\text { Facility Operation } \\
\text { w/ Ta-W (yrs) }\end{array}$ & 5.5 & 16 & 9 & 7 & 0 & NA \\
\hline $\begin{array}{c}\mathrm{Ta} / \mathrm{W} \text { Target Oper. } \\
\text { Life (yr) }\end{array}$ & 5.5 & 5 & $5 * *$ & $6+$ & TBD & $>10(\mathrm{tbd})$ \\
\hline Target Type & Stationary & Stationary & Stationary & Stationary & Stationary & Rotating \\
\hline Configuration & 4 plates & $\begin{array}{c}12 \text { plates; } 11 \mathrm{x} \\
12 \mathrm{~cm} ; \\
\text { Varying } \\
\text { thickness }\end{array}$ & $\begin{array}{l}\text { Single } 6.8 \\
\mathrm{~cm} \text { Dia. and } \\
30.7 \mathrm{~cm} \\
\text { long }\end{array}$ & $\begin{array}{l}\text { 7cyl. plates } \\
10 \mathrm{~cm} \mathrm{Dia}\end{array}$ & $\begin{array}{l}15 \text { plates } 13 \times 4 \\
\mathrm{~cm}\end{array}$ & $\begin{array}{l}16 \text { plates } \\
5 \mathrm{~cm} \text { thk }\end{array}$ \\
\hline $\begin{array}{l}\text { Ta Clad Thickness } \\
\text { (mm)* }\end{array}$ & .8 & 2.0 & 1.0 & .25 & .5 & 1.0 \\
\hline
\end{tabular}


Table 5.3. Summary of various tantalum clad tungsten target thermos-mechanical factors (continued)

\begin{tabular}{|c|c|c|c|c|c|c|}
\hline Facility & KENS & $\begin{array}{c}\text { ISIS TS-1 } \\
{[10]} \\
\end{array}$ & $\begin{array}{c}\text { ISIS TS-2 } \\
{[9]} \\
\end{array}$ & LANSCE & $\begin{array}{c}\text { CSNS } \\
\text { (unconfirmed) }\end{array}$ & STS \\
\hline $\begin{array}{l}\text { Max Water Vel. } \\
(\mathrm{m} / \mathrm{s})\end{array}$ & & 6 & 11 & 10 & 3 & 3 \\
\hline $\begin{array}{l}\text { Tungsten Max } \\
\text { Temp (C) }\end{array}$ & & 195 & 249 & 130 & $<100$ & $\sim 100$ \\
\hline $\begin{array}{l}\text { Tantalum Surf } \\
\text { Temp (C) }\end{array}$ & & 100 & 103 & 63 & $<100$ & $\sim 60$ \\
\hline $\begin{array}{l}\text { Tungsten Max } \\
\text { Stress (MPa) }\end{array}$ & & 106 & 150 & 103 (upper) & 79 & 62 \\
\hline $\begin{array}{l}\text { Tantalum Max } \\
\text { Stress (MPa) }\end{array}$ & & 116 & 90 & 174 & & 60 \\
\hline $\begin{array}{c}\text { Proton Pulse Rate } \\
\qquad(\mathrm{Hz})\end{array}$ & 20 & 40 & 10 & 20 & 25 & $.33 \mathrm{~Hz}$ \\
\hline $\begin{array}{l}\text { Peak Energy/Pulse } \\
\left(\mathrm{MJ} / \mathrm{m}^{3} / \text { pulse }\right)\end{array}$ & & $\sim 11$ & $\sim 70$ & & & 81 \\
\hline $\begin{array}{l}\text { Peak Heating } \\
\left(\mathrm{W} / \mathrm{m}^{3}\right)\end{array}$ & & $4.0 \mathrm{E}+08$ & $7.0 \mathrm{E}+08$ & $1.5 \mathrm{E}+6$ & $0.6 \mathrm{E}+08$ & \\
\hline
\end{tabular}

*Thinnest Section; ** Predicted

\subsubsection{Target operational characteristics}

Uniform distribution of the proton beam over the full circumference of the disk extends the operating life of the rotating target in comparison to a fixed assembly. This effect reduces the material damage rate on the stainless steel shroud to approximately $0.21 \mathrm{dpa} /$ year and limits temperature variations and cycles in the target blocks, thus reducing internal stresses. The reduced window dpa damage rate results in a theoretical increase in life from less than one year for a stationary assembly to more than 40 years in the STS target (Figure 5.37). The tungsten blocks experience internal stresses approximately $50 \%$ of 200 MPa operating limit [11]. The pulse rate exposure to individual target blocks is $0.33 \mathrm{~Hz}$, which ranges from $1 \%$ to $3 \%$ of the rates for proven stationary targets as show in Table 5.3. While all these factors argue for a potential target life of over 20 years, the STS conceptual design assumes an operational life of approximately 10 years for planning purposes. It should be noted that this assumption is not a design driver since maintenance planning and tooling is required regardless of the predicted life. 


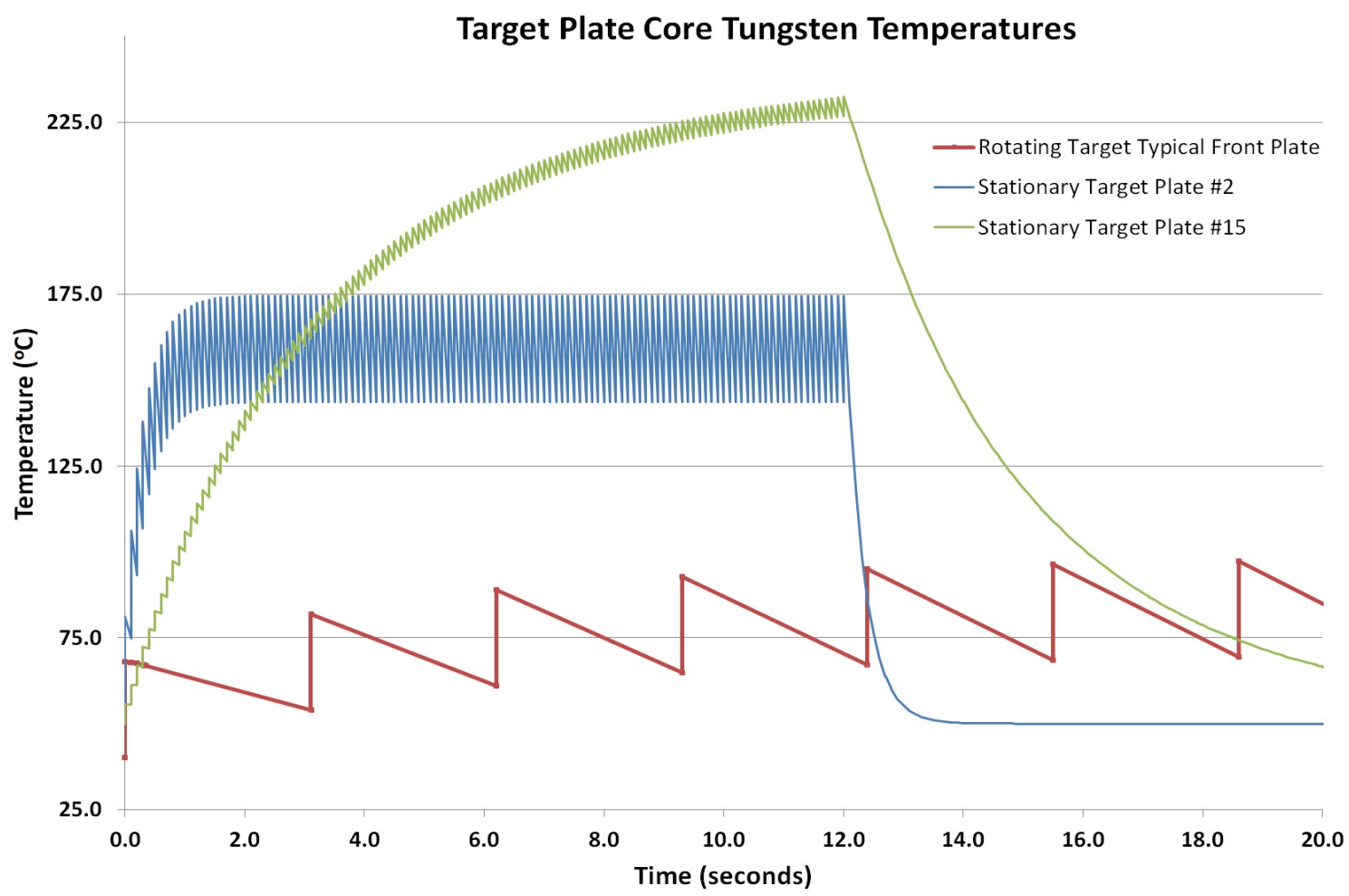

Figure 5.37. Comparison of peak plate temperatures in STS stationary target vs STS rotating target exposed to the same proton beam.

\subsubsection{Target fabrication}

All the currently operating tantalum-clad tungsten targets have been fabricated using the same basic process: HIP-bonding of the tantalum cladding on solid tungsten blocks. While there are differences in the details, most of the welding and processing methods are very similar due to limitations associated with the available fabricating tools.

It is recognized by the STS team that the proposed STS blocks are significantly larger than those used in the peak heating zone at other facilities. Therefore, a full-scale development program was initiated in 2016 to fabricate and analyze prototypical target blocks. The program is based on well documented procedures developed at ISIS [12], KENS [13], and LANSCE [14] but addresses the unique issues related to the size of the $41 \mathrm{~kg}$ (avg) STS blocks. Of interest are the weld shrinkage in the tantalum cladding and the uniformity of the tungsten block density. Proven vendors have been contracted to provide material and develop reliable fabrication techniques, particularly welding and cleaning. Later phases of the development program will focus on metallurgical considerations and large-scale production issues.

The basic manufacturing process proven to be successful has the following major steps. The development program will be used to detail the procedure for each of these.

- The target core will be 'pure' forged tungsten in accordance with ASTM B760-07.

- $\quad$ high purity (99\%) tantalum sheet in accordance with ASTM B708-05 will be used to clad the tungsten. The clad must be precisely fit to the tungsten blocks to avoid both trapped volumes and excessive stresses due to weld shrinkage. 
- Bonding surfaces on tungsten blocks and tantalum clad will be surface finished to $\sim \mathrm{Ra} 0.2$. HIP processing is normally performed at over $50 \%$ of material melting points. This is not possible for the STS blocks because the melting points of tantalum $\left(3020^{\circ} \mathrm{C}\right)$ and tungsten $\left(3422^{\circ} \mathrm{C}\right)$ exceed the maximum practical operating temperature of molybdenum HIP furnaces $\left(\sim 1400^{\circ} \mathrm{C}\right)$. Thus, the bonding temperature will be limited to $35 \%$ to $45 \%$ of melting. To help overcome this shortfall the bonding surfaces are polished to achieve maximum contact. This approach has been validated in testing at ISIS [15].

- Components will be cleaned prior to welding using conventional degreasing techniques combined with a final ultrasonic acetone bath. After cleaning maintaining clean surfaces will require special handling.

- EB welding will be used to seal the Ta clad around the tungsten block. Techniques to achieve high quality, uniform welds with adequate depth is a critical requirement since experience has shown that the welded joints are the most likely point of clad failure. The quality of the final seal will be tested using a conventional 'bomb' technique in which the assembly placed in a helium atmosphere then placed in a clean leak detection chamber where helium leaking out of the assembly can be detected.

- HIP Bonding in Molybdenum furnace will be used to anchor the clad to the tungsten. While the clad protects the tungsten from erosion it also must serve to conduct heat from the tungsten to the cooling water. The HIP 'recipe' has not been finalized, but it will closely follow the proven formula used by other facilities. Temperature is increased inside the pressurized vessel (150 to $200 \mathrm{MPa})$ at a rate of approximately $\sim 10^{\circ} \mathrm{C} / \mathrm{min}$ to a maximum of 1200 to $1400^{\circ} \mathrm{C}$ where it is held for $3-4 \mathrm{hr}$. The furnace is pressurized with $5 \mathrm{~N}$ Argon; which is de-oxidized inside the furnace with getter material surrounding the target block.

- Ultrasonic NDT has proven to be an effective method of assessing the quality of diffusion bond. It will be used on the STS blocks.

\subsubsection{Target development}

Early second target station studies begun in 2007 explored the benefits of a rotating target and prompted the development of a full-scale prototypical assembly (Figure 5.38) [16]. The prototype includes an independent overhead drive, water seals and suspended disk similar in design to that proposed for STS. The facility has been operated over 6000 hours without maintenance intervention, leaking or operating difficulty. Most importantly, it demonstrated that a suspended, rotating target can be fabricated and operated with a disk runout of less than $0.5 \mathrm{~mm}$. 


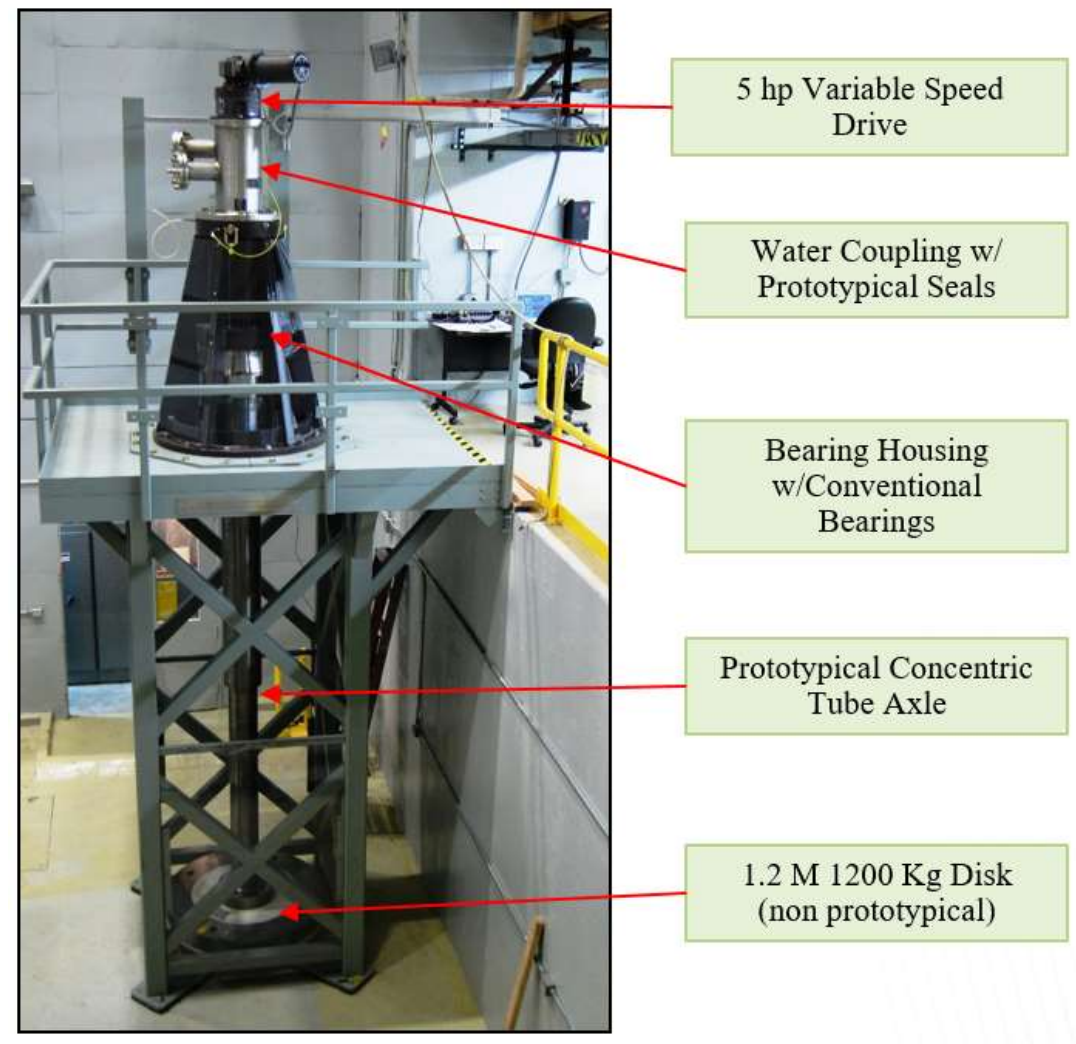

Figure 5.38. Full scale prototypical rotating target assembly.

\subsubsection{Target maintenance}

Change-out of spent targets will be very difficult and time consuming (three to four weeks). Even though the basic techniques of vertical removal are fully developed, the size of the target disk and residual heat removal (Figure 5.39) will be a challenge. Further, the moderator-reflector plug must be moved to clear the disk for vertical handling. This will involve removal of the periscope plug and disconnection of numerous cryogenic and cooling water pipes. If the target lasts the full design life of 10 years or longer, this will not be considered significant since it can be coupled with a normal moderator-reflector plug replacement. However, a premature target failure will require a significant operating schedule upset. 


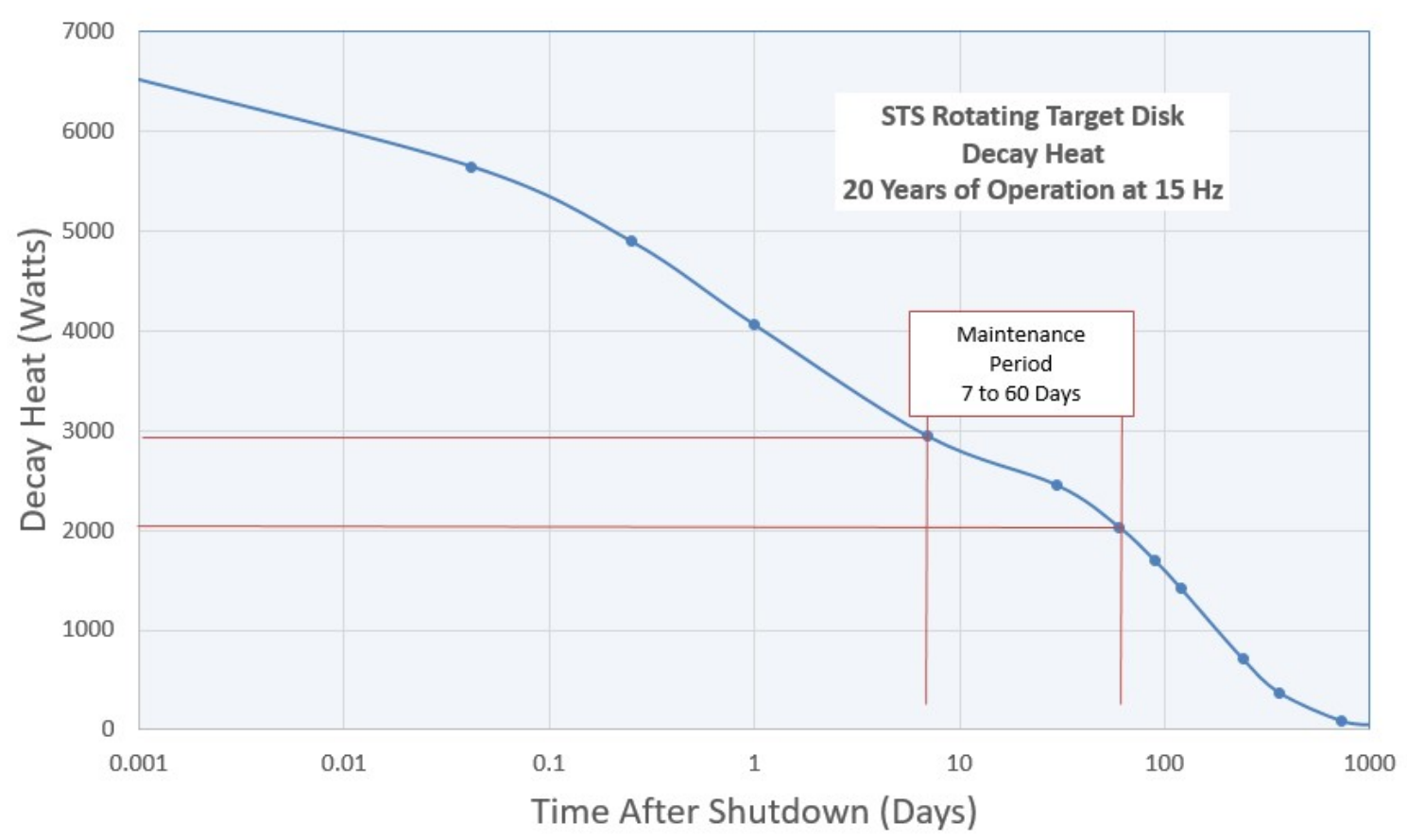

Figure 5.39. Target disk decay heat after 20 years of $15 \mathrm{~Hz}$ beam operation.

Failure modes for the target can be grouped into three categories; drive system, shroud/axle and target blocks. The drive system includes all active components including the motor, reducer, water seals, and all sensors (bearing vibration, rotation, temperature, and leak). It will be accessible for hands-on maintenance during beam-off shutdowns. The complete drive assembly will be packaged for replacement of individual components or as a unit without having to remove the rotating target axle and disk. The shroud and axle are heavy-walled, passive structures with a low probably of failure; therefore, they are packaged with the tantalum-clad tungsten blocks.

Since tantalum-clad, tungsten targets have experienced some cladding failures that resulted in tungsten erosion, provisions are made in the return water path to monitor and remove contamination before it spreads through the complete water loop. As demonstrated at other facilities, this will allow the STS target to continue to operate with nominal cladding failures until a reasonable change-out shutdown can be scheduled.

\subsubsection{Target and Monolith response to loss of cooling with decay heat}

A simplified 2D axisymmetric model of the target and monolith was done to estimate the temperature rise due to a loss of cooling with decay heat. Figure 5.40 shows the development of the model used in Heating 7 from an early ProE model. The majority of the target disk is within a stainless steel shielding block. The axisymmetric model assumes the entire target has this configuration and does not include the smaller zone within the moderator/reflector assembly. Gap regions were modeled to account for heat transfer by thermal radiation and gas conduction. The outer concrete ring beyond the $4.5 \mathrm{~m}$ iron shielding radius was not included. The target decay heat and peak temperature versus time were shown in Figure 5.23. Figure 5.41 shows the overall temperatures when the peak occurs at 11.1 hours and after 30 days. 


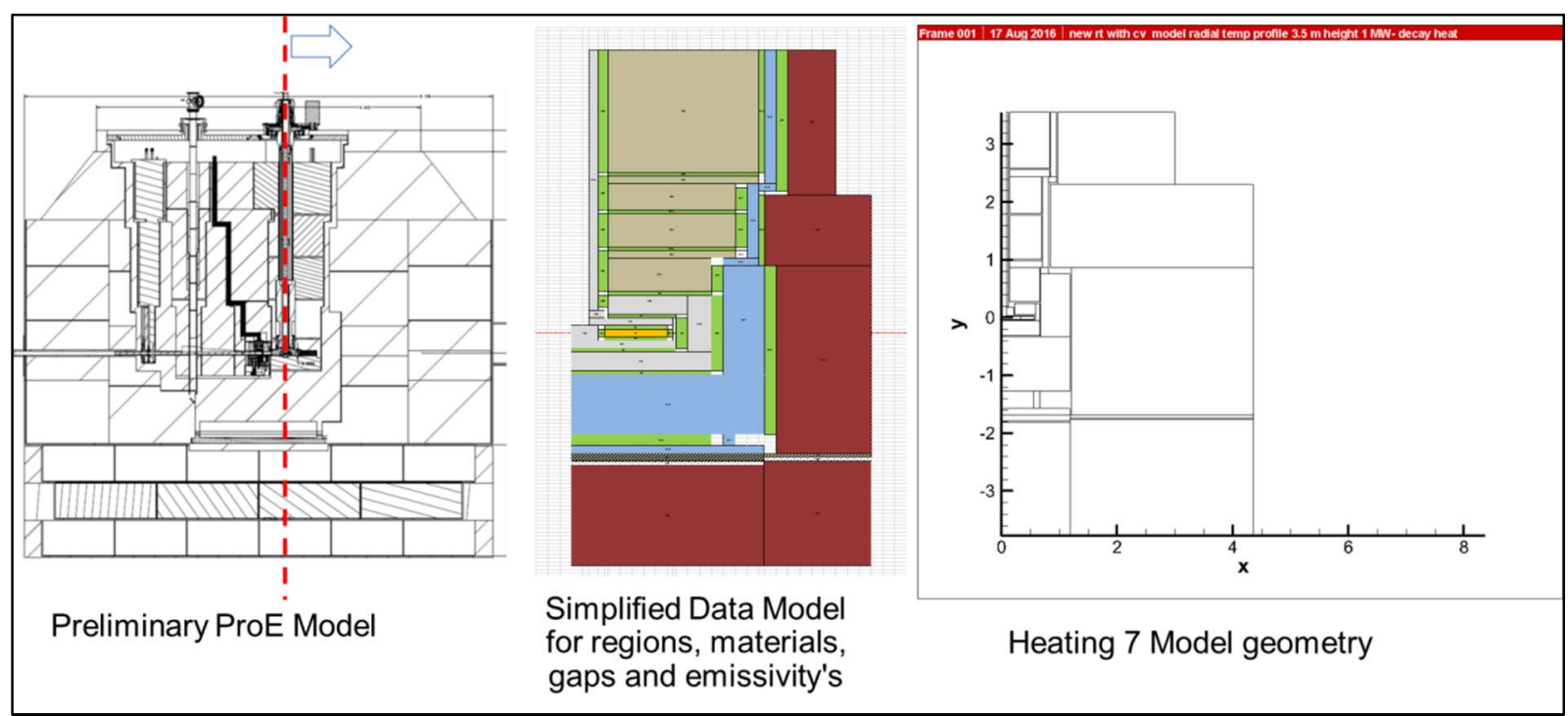

Figure 5.40. Target and Monolith Heating7 analysis model for loss of cooling with decay heat.
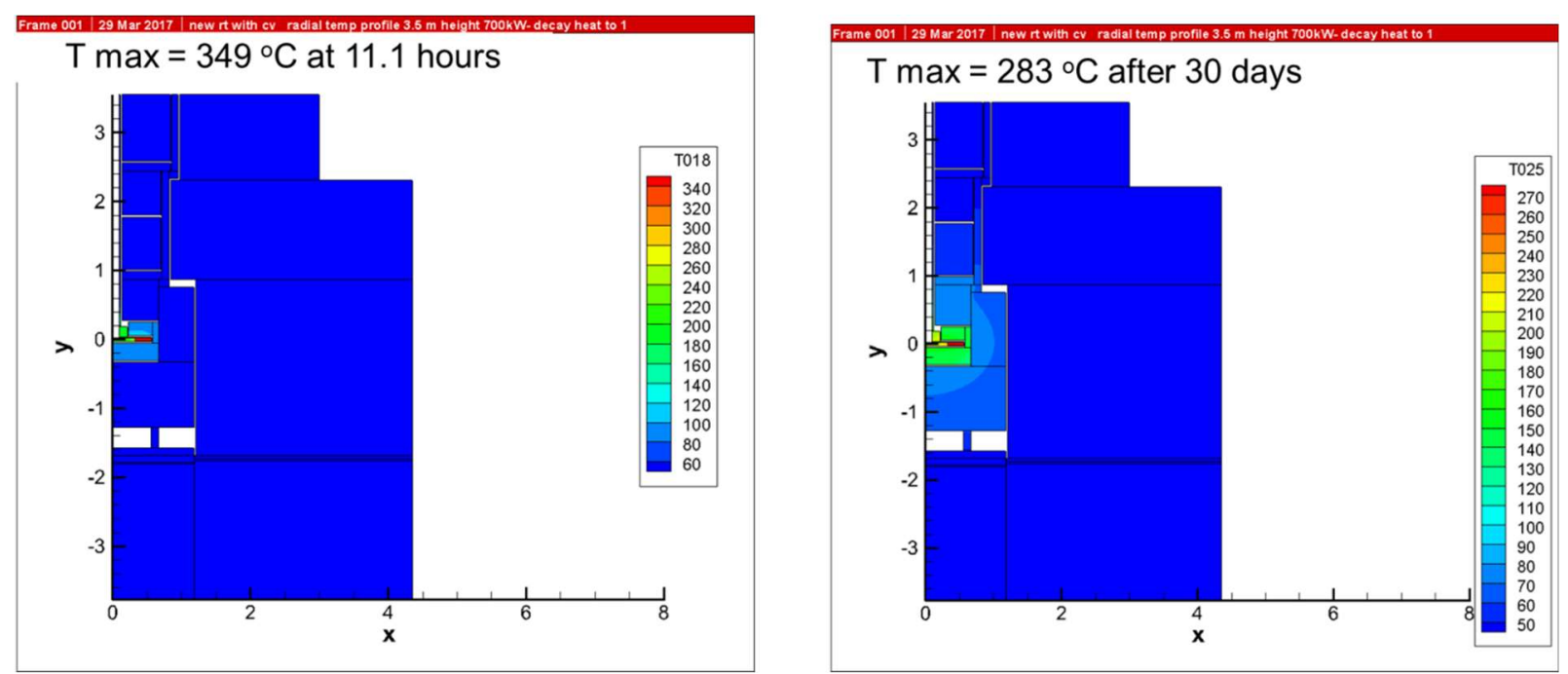

Figure 5.41. Peak temperatures at 11 hours and after 30 days.

The thermal response of the target for a month is dominated by the thermal mass of the target and also the shielding within about a 1 meter radius. Figure 5.42 shows the region around the target when the temperature peaks and indicates that the first shielding block around the target is an important heat sink. 


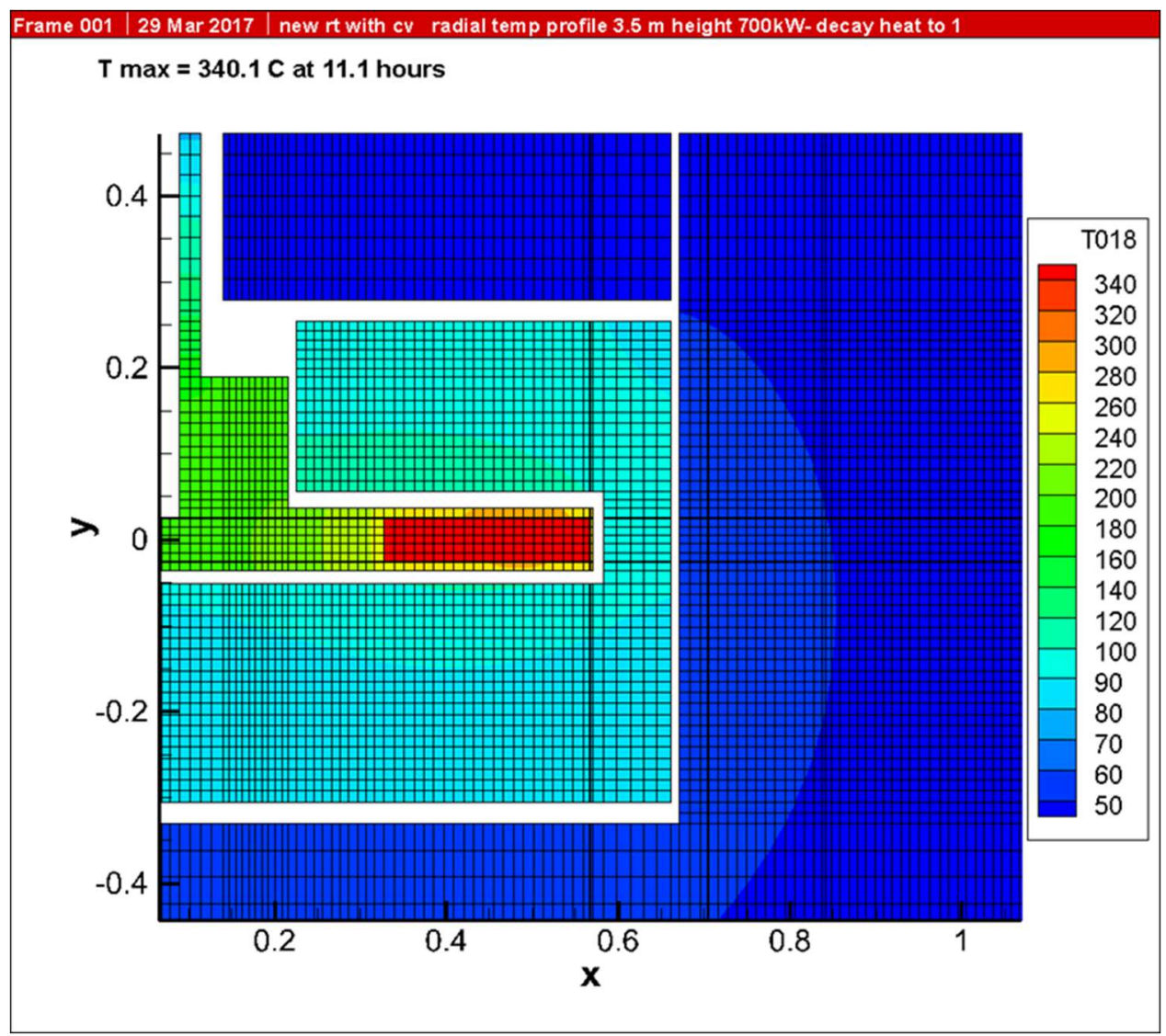

Figure 5.42. Peak temperatures around the target disk after 11 hours.

Thermal radiation to the shielding is the dominate heat transfer mechanism from the target disk. The overall response is very slow and the peak is still decreasing after 30 days. There is a large margin between the peak temperatures and the $800{ }^{\circ} \mathrm{C}$ threshold for tungsten vaporization.

\subsubsection{Periscope}

SNS operating experience and the desire for accurate alignment of the core components led to the requirement for an in-situ viewing system. The FTS target viewing team was enlisted to design and develop a robust periscope capable of providing continuous beam-on viewing of the target. This team determined the best approach to be a simple arrangement based on two flat mirrors and commercial optics positioned outside the target drive bunker to facilitate maintenance during beam-on operations. The resulting configuration (Figure 5.43) effectively means the camera has a telescopic view from approximately 10 meters. Additional shielding analysis may ultimately show the need for a third mirror outside the bunker to protect the cameras, but this is not expected to significantly degrade the image. 


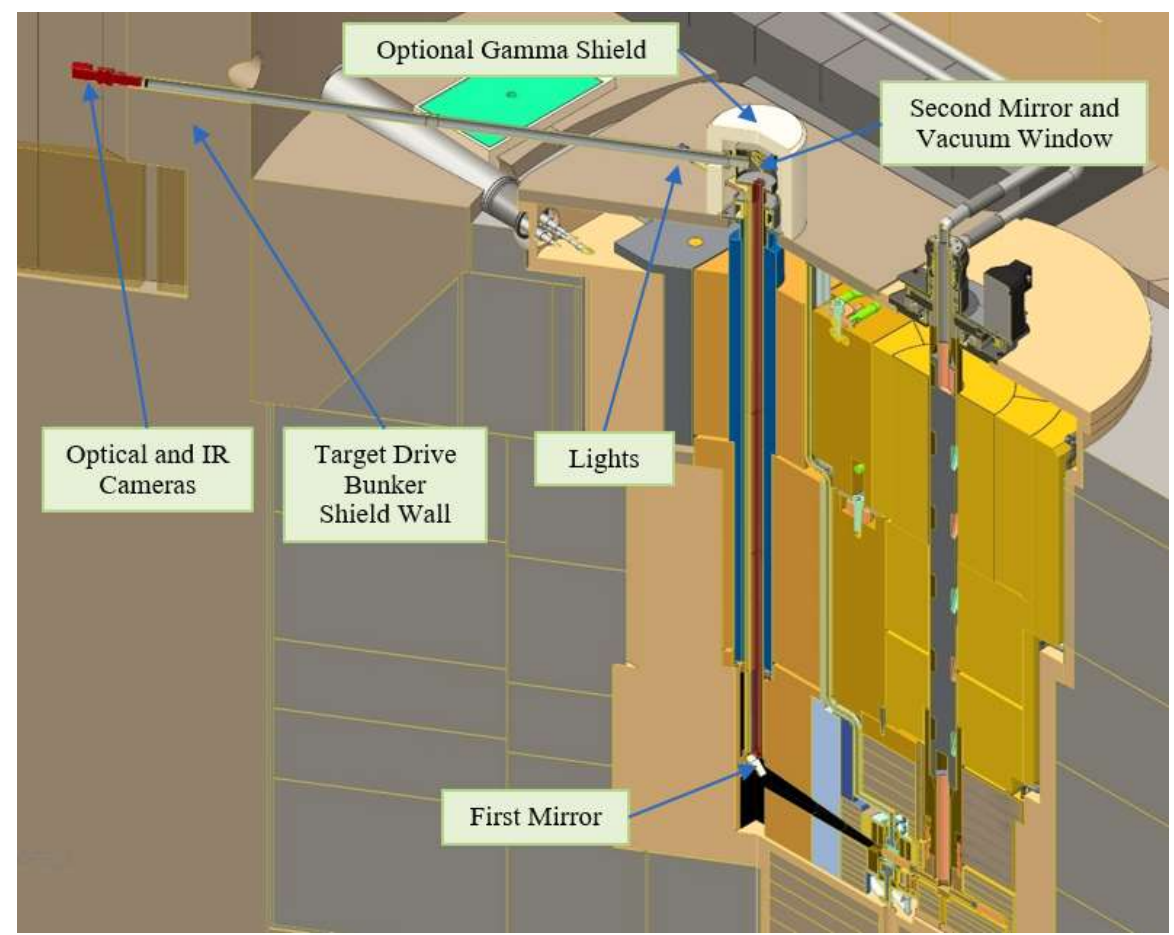

Figure 5.43. Section target monolith along periscope sightline.

The system will have two visible light viewing modes and one IR spectrum mode. During alignment and inspection, the target area including certain critical moderator-reflector and vessel interfaces will be illuminated by lights located in the target drive bunker. Two polished pipes will transmit the light to a reflective surface above the optic mirror that will illuminate the target area (Figure 5.44). During beam-on operation, the proton beam incident spot on the target will be made visible by a luminescent coating with embedded fiducials. Thermal imaging will be made possible with a dichroic beam splitter, IR optics and an IR camera. This simultaneous imaging will allow monitoring of the uniformity and consistency of the target nose temperature, which is an indicator of possible water distribution problems inside the target.

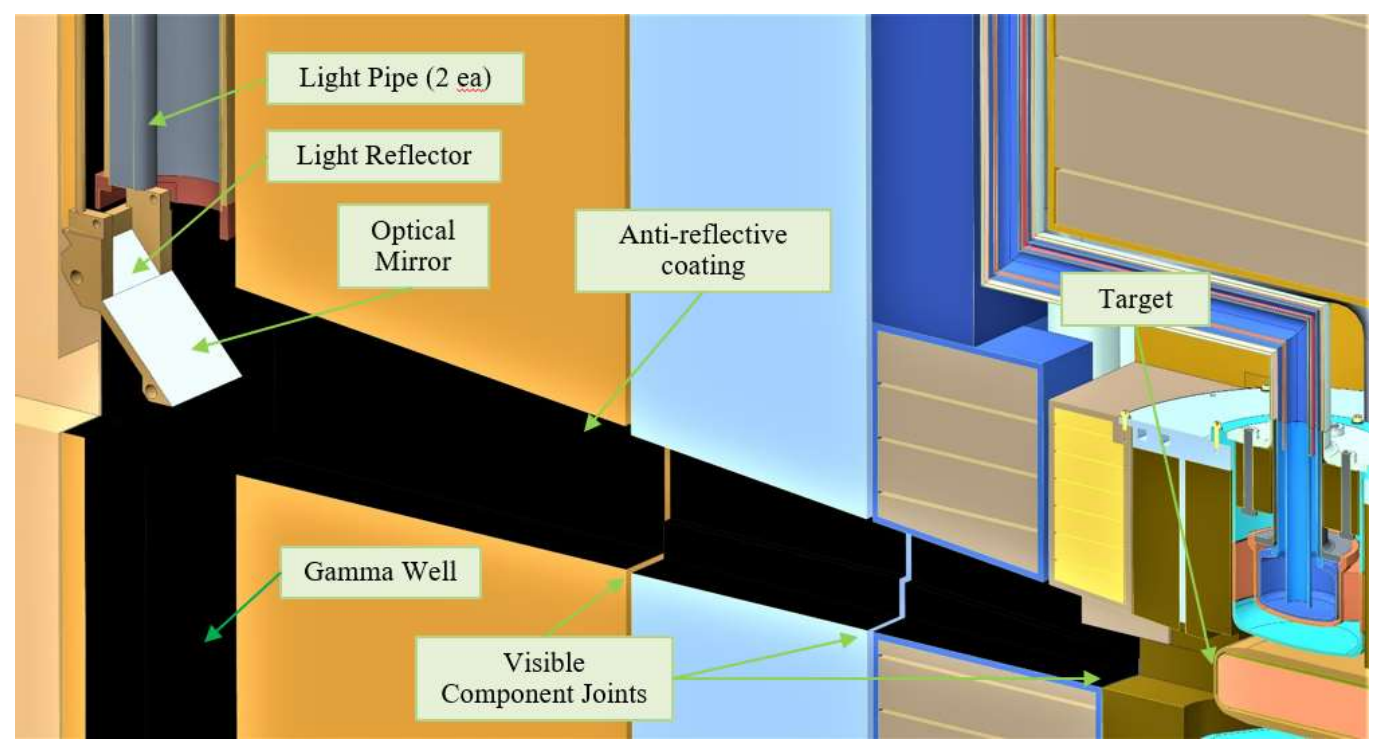

Figure 5.44. Detail of lower periscope configuration. 
Over the last year, a full-scale mockup of the proposed optical system has been constructed at ORNL (Figure 5.45). The mockup has demonstrated that the desired capabilities can be achieved; most importantly, tests have shown that target area features can be discerned with less than $1 \mathrm{~mm}$ resolution (Figure 5.46). This level of precision allows the periscope to monitor the position of the target and to assist with proper alignment of replacement moderator/reflector and target assemblies. It has also been demonstrated that atmospheric distortions will be minimal if the vessel must be operated with a subatmospheric helium environment rather than vacuum. It should be noted that the IR thermal imaging and IR spectroscopy capabilities have not been tested in the mockup system since they are well established using, commercially available products. Also, the current baseline calls for the use of the FTS luminescent coating even though improved coatings may become available through the on-going development program underway in conjunction with the ESS project.

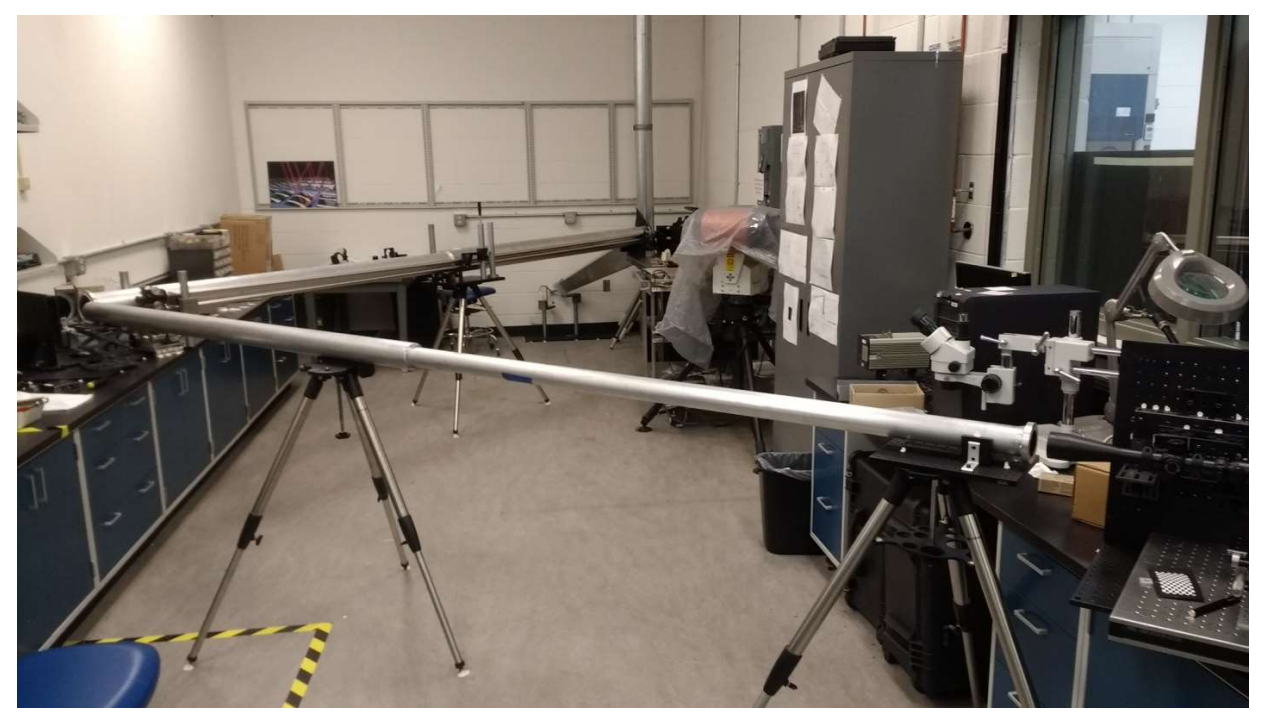

Figure 5.45. Periscope mockup facility.

Object 1

(25" in front of target)

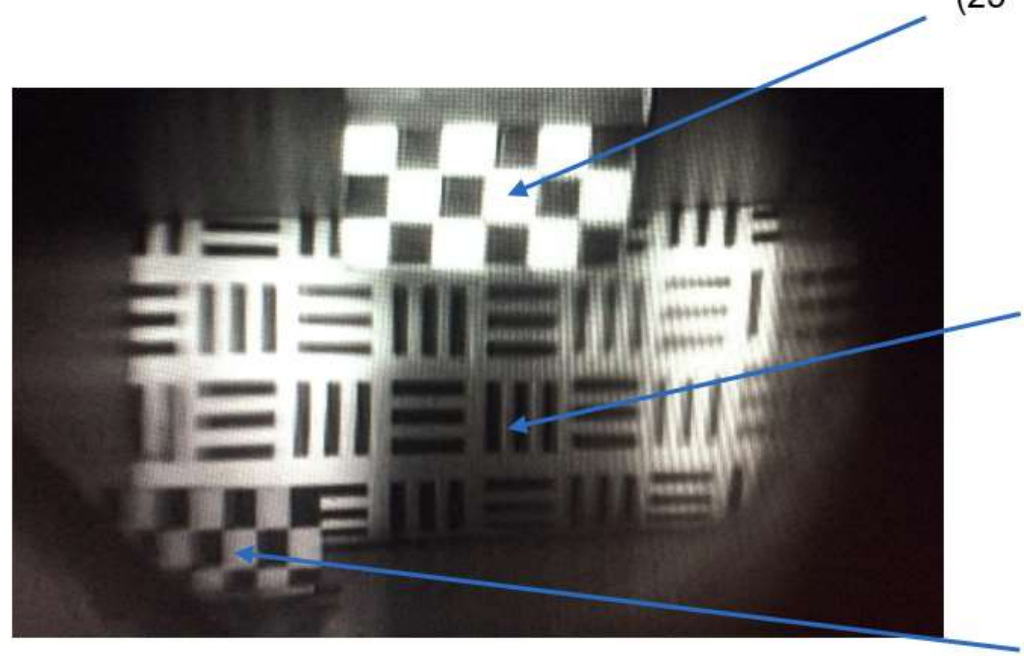

Target Face
(shadowed by object 1)

Object 2

(10" in front of target)

Figure 5.46. Mockup periscope target image showing resolution and depth of field. 
The water cooled first mirror is the only uniquely new element of the proposed periscope. While all other components in the system are commercially available and can be maintained and aligned hands-on, the first mirror must be accurately aligned in the core vessel mockup test stand prior to assembly. The reflective surfaces of the first mirror must be protected against corrosion in the event of a small water leak inside the vessel by gold plating the critical surfaces. This will result in a different attenuation and optical spectrum which might need to be considered along with adjustments to improve IR temperature measurements. The mirror must also be uniformly cooled to maintain reflective surface flatness and thus the quality of the image. The neutronic analysis indicates that the mirror will be heated at a rate of approximately $0.003 \mathrm{~W} / \mathrm{cc}$. Since this is an idealized number, a finite element analysis was performed at $0.02 \mathrm{~W} / \mathrm{cc}$ to account for stray proton beam, radiant heating, and to add conservatism. As shown in Figure 5.47 , this analysis indicates a maximum mirror temperature of approximately $23^{\circ} \mathrm{C}$; this amount of heating can be controlled with less than $0.1 \mathrm{l} / \mathrm{s}$ of water flow. The calculated deflection on the order of $10^{-}$ ${ }^{3} \mathrm{~mm}$ should be within the tolerance required to maintain an acceptable image. It should also be noted that the design includes additional thermal control by holding the cooling water inlet temperature to a band of $\pm 1^{\circ} \mathrm{C}$. A functional prototype of this component is planned for future addition to the periscope mockup to confirm the optical, thermal and mechanical performance of the proposed arrangement.
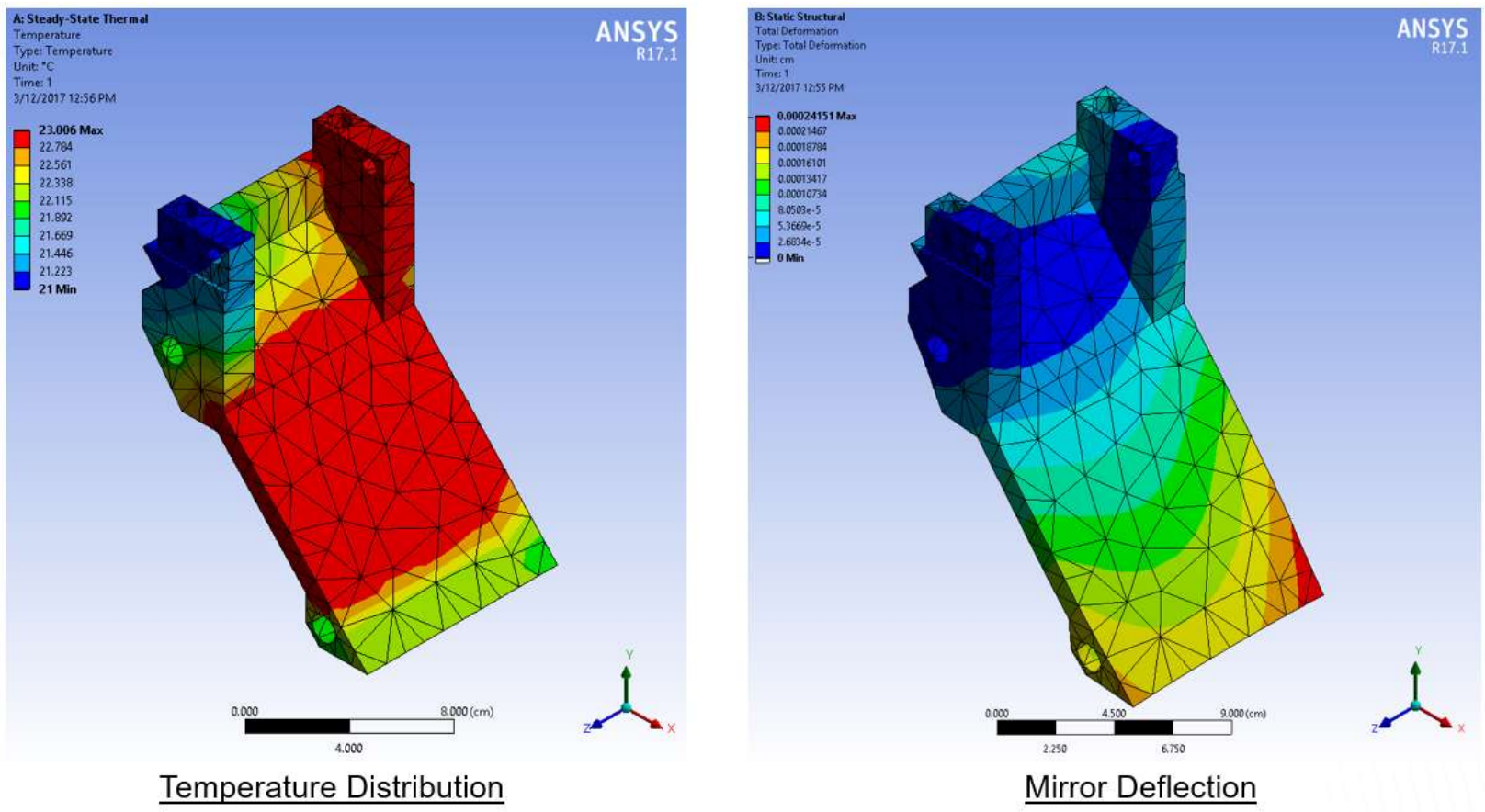

Figure 5.47. Temperature distribution and deflection in first periscope mirror during beam-on operation.

\subsubsection{Proton Beam Window and Halo}

Proton beam windows (PBWs) are a proven technology used at all accelerator-based neutron-scattering facilities. STS will use the typical configuration based on a thin water-cooled window mounted at the bottom of a vertical plug and sealed to the proton beam tube with an inflatable metal bellows seal. The assembly will also include a proven thermocouple-based Halo monitor downstream of the window. An aluminum window will reduce beam scatter compared to the alternative Inconel. Aluminum 6061-T6 is currently being tested in FTS where it will be fully validated prior to use in STS.

Neutronic analysis data has been produced to provide heating and dpa damage information for use in the window design (Figure 5.48). 


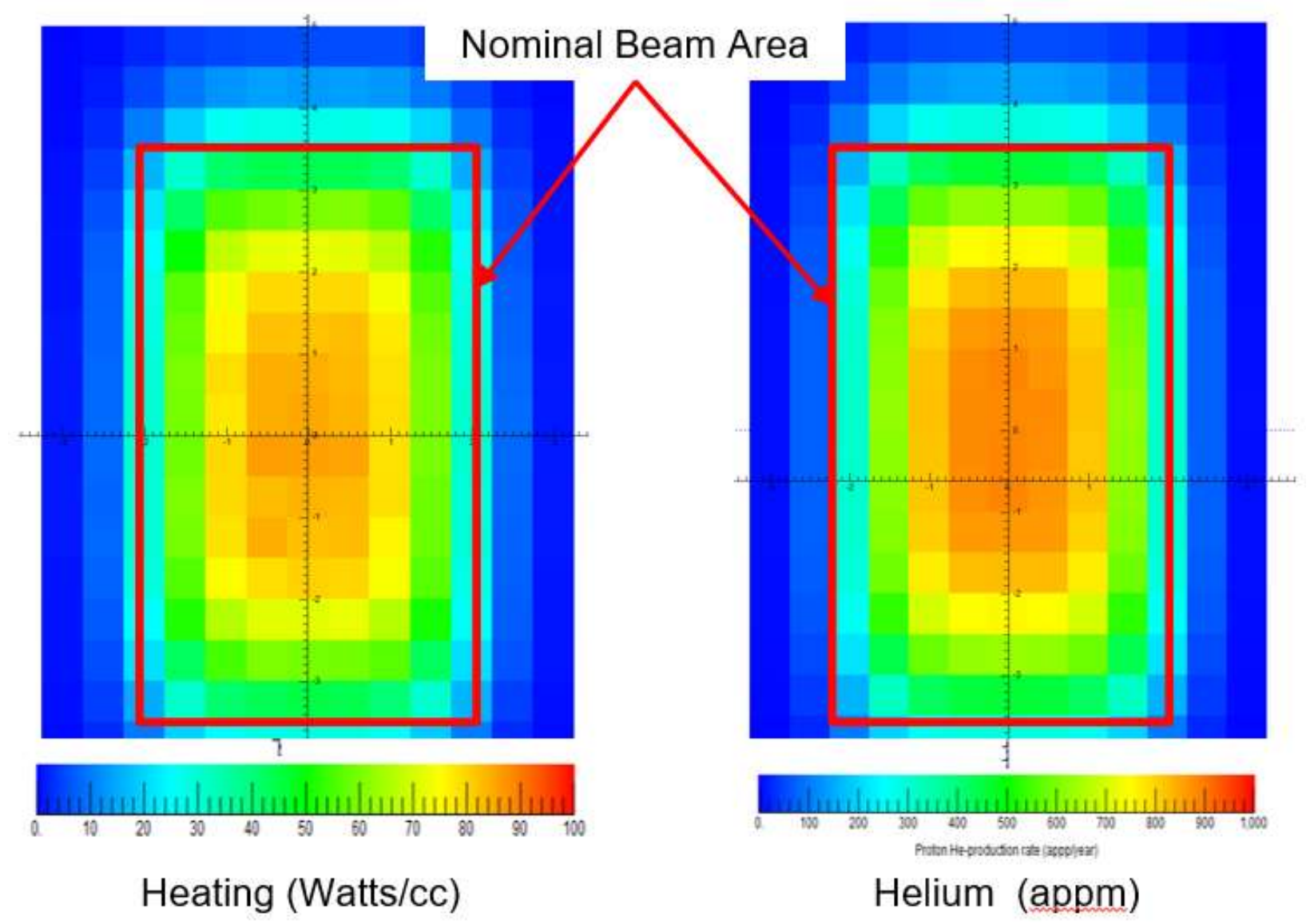

Figure 5.48. Proton beam heating and helium in aluminum proton beam window based on a $10 \mathrm{~Hz}$ beam.

The neutronic analysis of the proton beam window estimates a damage rate of approximately $1.1 \mathrm{dpa} / \mathrm{year}$ at $15 \mathrm{~Hz}$, which would yield a lifetime of over 9 years if a commonly applied damage limit of 10+ dpa is used. On the other hand, if the more conservative lifetime limit of 2000 appm helium is used, then the calculated production rate of $1335 \mathrm{appm} / \mathrm{yr}(15 \mathrm{~Hz})$ will limit the life of the window to 1.6 years. Given this relatively short life, STS is evaluating two options. First, if operational experience with 6000- and 5000 -series aluminum alloys is considered, the life of the window should be based on dpa damage and can be expected to last several years [17][18]. If this approach is extended to include the possibility of a window failure, then water entering the accelerator would have to considered an acceptable trade-off risk. In the second option, which could also possibly be used to extend window life, the window assembly could incorporate multiple window positions with an in-situ ability to shift between each without significant disassembly effort or time. The proposed multiple window arrangement is shown in Figure 5.49. Obviously, if one of the windows fails, the assembly would have to be changed. It should also be noted that Halo monitor assemblies have proven to be highly reliable; however, a very long-lived PBW could challenge this limit. Additional analysis of this subject is required to ensure the two systems can be compatibly packaged in the same module.

The current STS design has a side stream of the target cooling water routed through the PBW. If the target cooling water flow stops due to a process system failure, the immediate result will be a window burnthrough that will "spoil" the proton beam with escaping water and thus protect the target from permanent damage. 


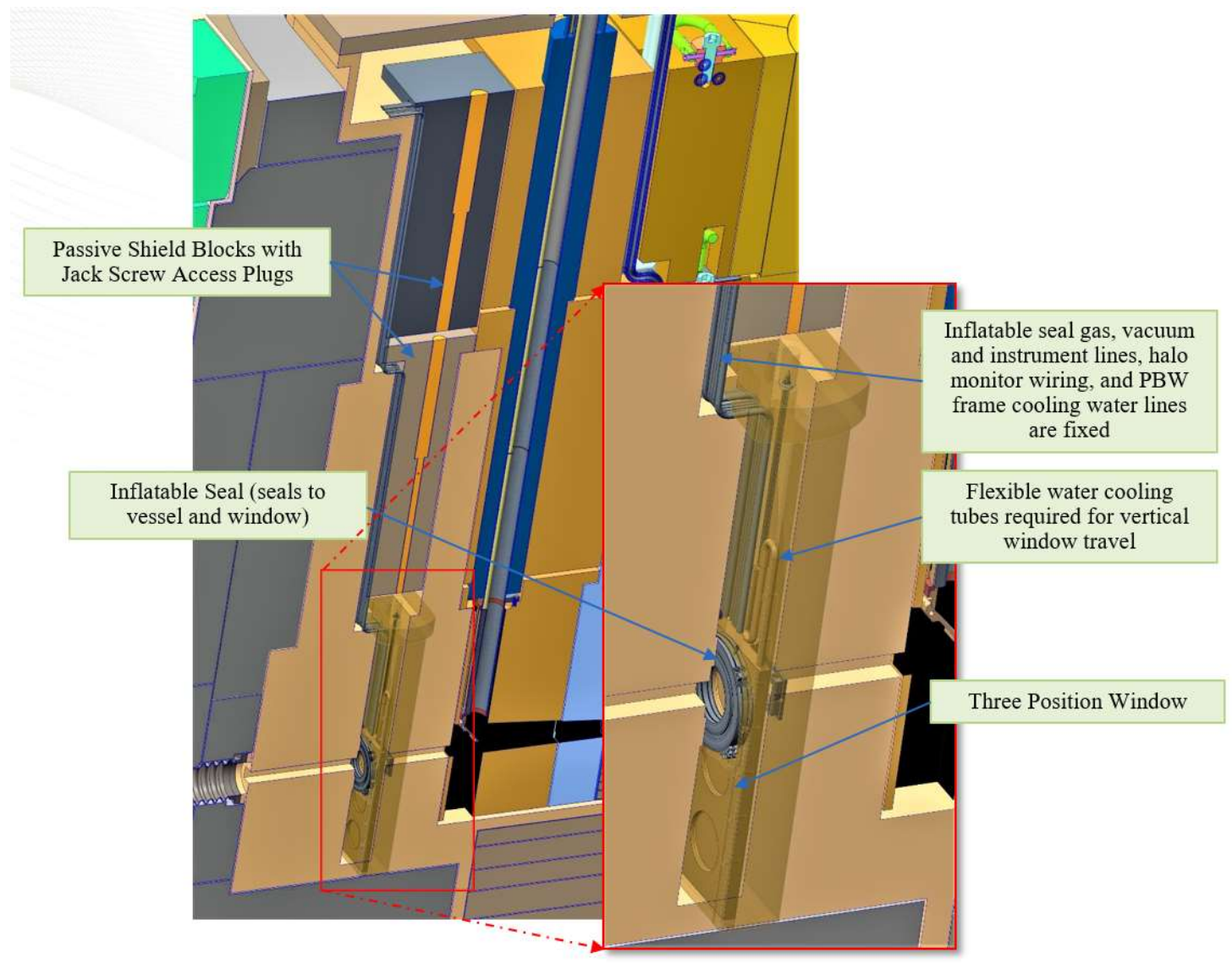

Figure 5.49. Proton beam window configuration.

\subsubsection{Proton Beam Wire Harp}

STS will have a wire Harp similar to the system used in FTS (Figure 5.50) and other accelerator facilities. As noted in the Section 5.4.6.1, the Harp is the least precise device of the alignment sensors; therefore, STS plans to use this system as a reliable backup to the Halo and periscope proton beam positioning systems.

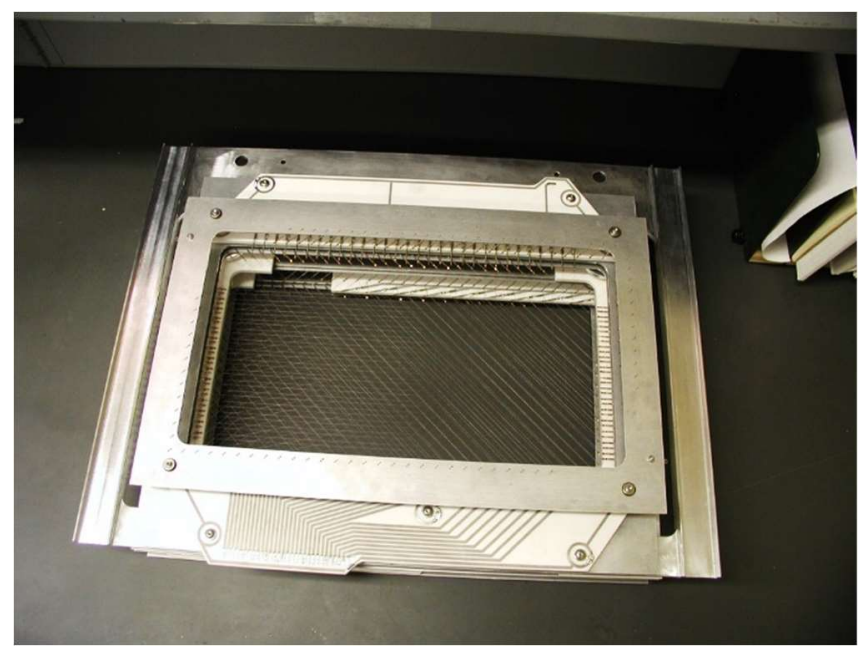

Figure 5.50. Current SNS proton beam Harp module. 
The FTS Harp has proven to be highly reliable, having remained continuously in the proton beam for 11 years to date. However, the STS proton beam flux density is $440 \%$ higher, so the operating life of the same basic system could be significantly shorter. Consequently, the proposed STS Harp may incorporate a retraction mechanism to reduce beam exposure to ensure an extended life. Regardless of the actual assembly operating life (assumed to be 5 years for planning purposes), the Harp assembly is designed to be efficiently changed using the proven vertical shielded container arrangement described in Section 5.5.2.

\subsection{NEUTRON BEAM LINES}

The twenty-two STS neutron beam lines interface with several target system components, starting with the moderators and passing through the core vessel, monolith shield and into the beam line bunkers. This section describes those beam line components and how precision alignment between them is accommodated by their design and subsequently maintained.

\subsubsection{Monolith Inserts and Neutron Guide Modules}

Neutronic performance improvements in STS rely on the use of elliptical focusing mirrors, precision alignment of guide components, and minimizing the number of windows in the guides. All three requirements argue for long, purpose-built, continuous guides in the monolith. Locating the initial beam guides as close as possible to the moderators, well inside the monolith shielding radius, to maximize the acceptance angle is a primary instrument and target station design requirement. A complicating requirement resulting from the close proximity of the guides to the target is beam heating.

To address all these requirements, STS will have a three-layer guide assembly (Figure 5.51). Because the core vessel is not repairable and locating beam windows on close radius flanges is not feasible, nozzle extensions are permanently attached to the core vessel to extend the vessel environment to a location outside the monolith shielding which is accessible. This provides for a remotely-replaceable structural monolith insert to be installed inside the nozzle and sealed to the outside flange of the nozzle. The active monolith inserts will be water cooled and have aluminum beam line window at both ends. Due to their estimated size $(\sim 4.5 \mathrm{~m})$ and weight ( $\sim 2$ tons), it is preferred to install the monolith inserts during facility construction rather than when beam lines are installed. Their reentrant design accommodates installation and removal of optical guide modules or shielding modules without breaking their vacuum seals to the nozzles. Upstream windows pre-installed on each monolith insert are expected to be life-of-facility components and are not replaceable since they would be highly activated; however, if a failure occurs, the entire insert can be replaced. 


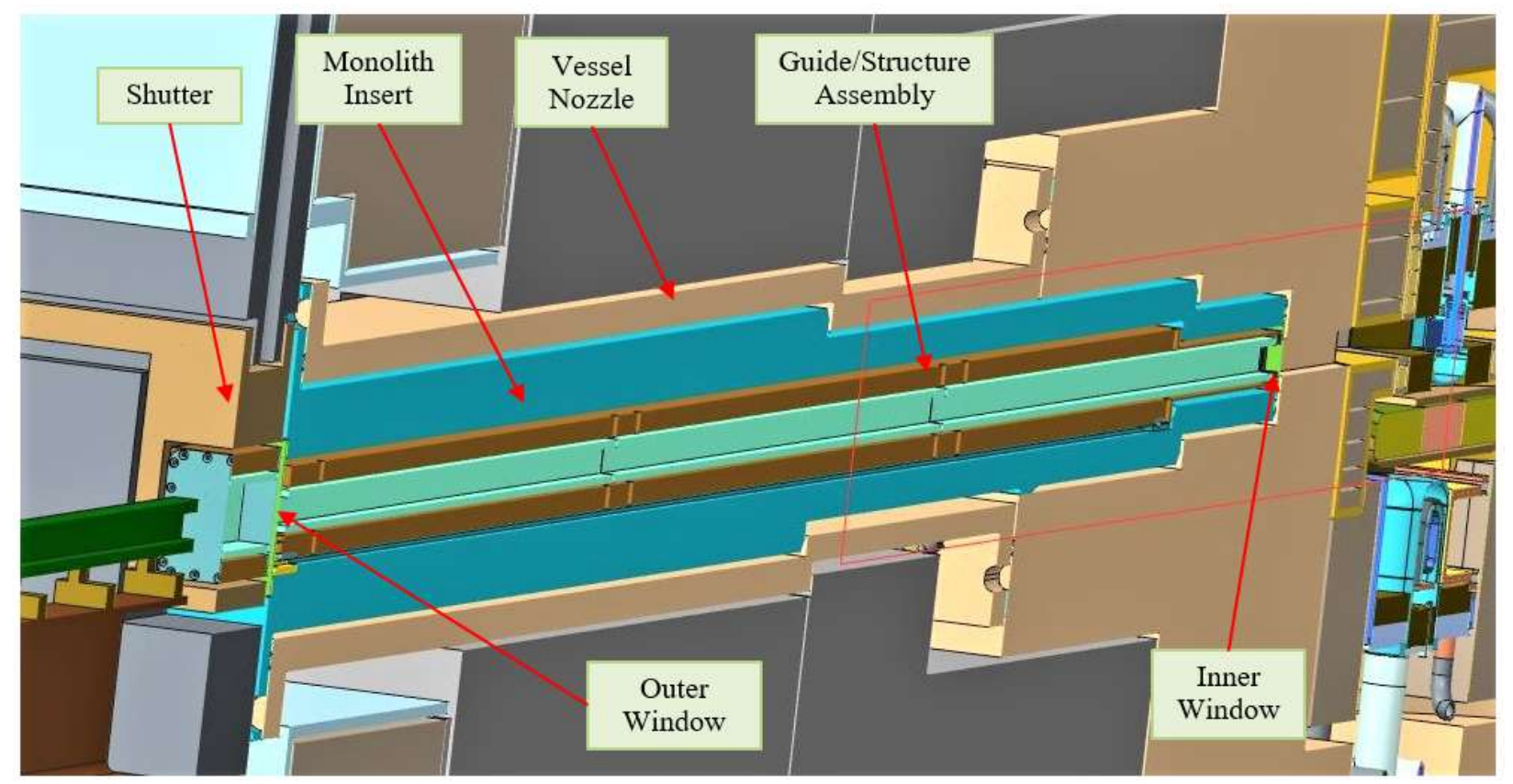

Figure 5.51. Typical neutron beam line monolith insert with guide module.

Instrument-specific guide modules will be inserted into the monolith inserts. Guide cooling is provided by convective helium cooling inside the monolith insert. Monolith insert and guide module lengths are driven by the monolith shielding radius and are approximately $4.5 \mathrm{~m}$ long. Additional neutronic analysis is required to determine the ideal configuration of monolith shielding; while the shielding monolith's diameter can increase, it cannot be significantly reduced since sufficient perimeter must be available to fit the maintenance shutters into the structure.

The use of monolith inserts within the monolith shielding allows beam guides to come within 70 to $100 \mathrm{~cm}$ of their respective moderator, depending on interferences with neighboring inserts or the rotating target disk (see Figure 5.52). Insert width significantly impacts the beam guide approach distance to the moderators, and the shape of the insert walls has been considered in some detail to minimize their overall width while still providing adequate thickness to accommodate internal water cooling channels in the most upstream portion of the inserts. The inserts are sized based on their moderator view $(3 \times 3,5 \times 5,3 \times 6$, or $7 \times 7 \mathrm{~cm}$ ) and port position. It was initially hoped that a common insert size could be used for all beam ports, but minimizing the approach distances for all inserts required that they be sized according to their moderator view. As beam line designs evolve, it is anticipated that some customization may be required for specialized optical requirements, but at this time all inserts with the same moderator view are identical. 


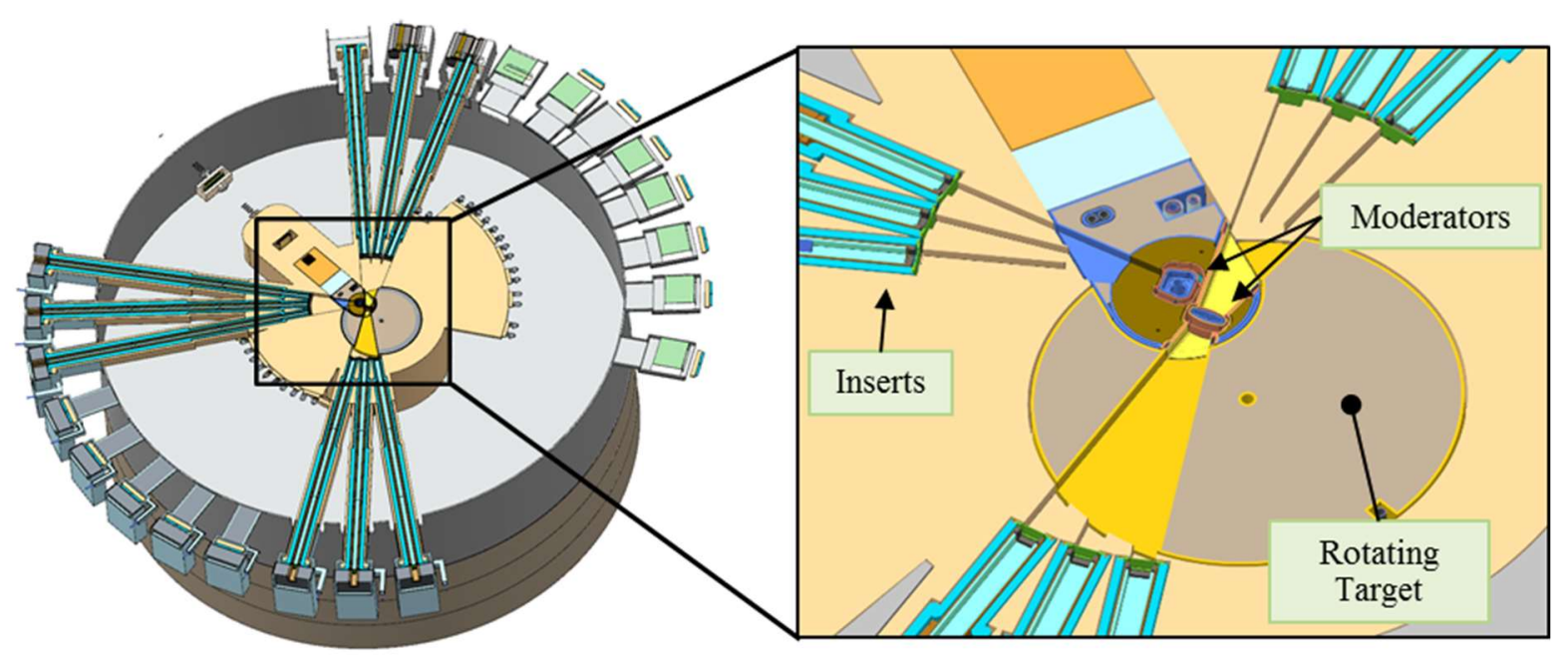

Figure 5.52. Monolith inserts viewing the two lower moderators.

Because all the inserts are installed during initial construction, the sizing of the reentrant openings must make assumptions and limits regarding the optical requirements of future instruments. A goal will be to reasonable excess space for larger future optics, but because the array of inserts is width-constrained, providing additional vertical space within the inserts will be simpler than providing horizontal space. During facility construction, each insert will be filled with either an operational guide module or a shielding plug. As future beam lines are installed, the shield plugs will be replaced with purpose built guide modules by removing the downstream insert window. Figure 5.53 shows an optical guide module being installed within a monolith insert. This operation must be performed at least semi-remotely because the moderators and target will still be in place as future beam lines are installed. The guide modules will have kinematic locating features which engage matching internal insert features using a simple pushing motion during guide installation; gravity holds the guide modules in position once the kinematic features have engaged. These features are described in more detail in Section 5.4.6.4. Initial concepts for the remote handling equipment for both the inserts and guide modules have been developed as described in Section 5.5.2.

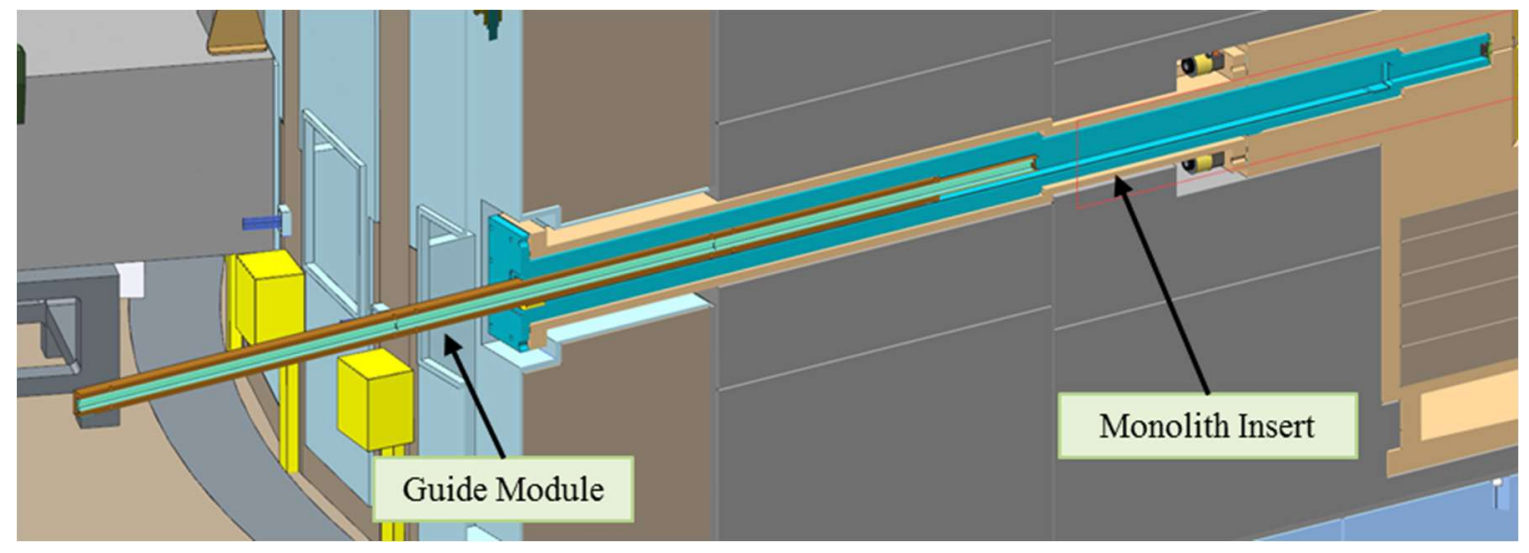

Figure 5.53. Guide module being inserted into a monolith insert. 


\subsubsection{Shutters}

Providing the ability for hands-on maintenance and assembly activities within the bunkers is a primary facility design requirement, which requires that equipment in the bunkers must be configured or locally shielded to allow such access, even after years of facility operation. To shield personnel from gamma radiation emanating from the target area and passing through the monolith along the neutron beam lines, relatively short ( $\sim 20 \mathrm{~cm}$ tungsten) shutters are provided to isolate the bunkers only during beam-off maintenance operations. These "maintenance shutters" (Figure 5.54) cannot be used as neutron blockers since even relatively short exposure to neutrons will activate the tungsten gates to levels which will preclude human access. Instead, each beam line will individually provide "operations shutters" or other features to support experimental operations. The FTS experience supports this decision since, as Table 5.4 shows, only a few FTS beam lines use their near-target primary shutter for experimental operations and that most lines use the primary shutters for weekly- or run-cycle-shutdowns only. All other FTS beam lines have sufficient curvature or a secondary shutter to support normal operations.

Table 5.4. FTS shutter use during beam-on operations between Jan 21 and Apr 21, 2015 ( 13 weeks).

\begin{tabular}{ccc}
\hline $\begin{array}{c}\text { FTS } \\
\text { Beam Line }\end{array}$ & $\begin{array}{c}\text { Primary Shutter } \\
\text { Operations }\end{array}$ & Ops/Week \\
\hline 3 & 347 & 26.9 \\
18 & 133 & 10.3 \\
12 & 94 & 7.3 \\
17 & 57 & 4.4 \\
5 & 37 & 2.9 \\
14 & 13 & 1.0 \\
13 & 11 & 0.9 \\
1 & 7 & 0.5 \\
2 & 6 & 0.5 \\
4 & 6 & 0.5 \\
16 & 3 & 0.2 \\
6 & 1 & 0.1 \\
7 & 1 & 0.1 \\
11 & 1 & 0.1 \\
15 & 1 & 0.1 \\
\hline
\end{tabular}

As shown in Figure 5.54, the maintenance shutters have three separate regions that can be positioned within the beam line: an optional optical guide with kinematic locating features, a neutron absorber that is used for reducing activation of a failed downstream component such as a chopper located between the maintenance shutter and an operations shutter, and a gamma blocker that is used during facility maintenance periods. Prior to personnel accessing the bunker, all maintenance shutters will be placed into gamma blocking mode.

It is also noted that maintenance shutters do not have to be installed for unused beam ports with shielding monolith inserts but will be added as neutron beam lines are installed. 


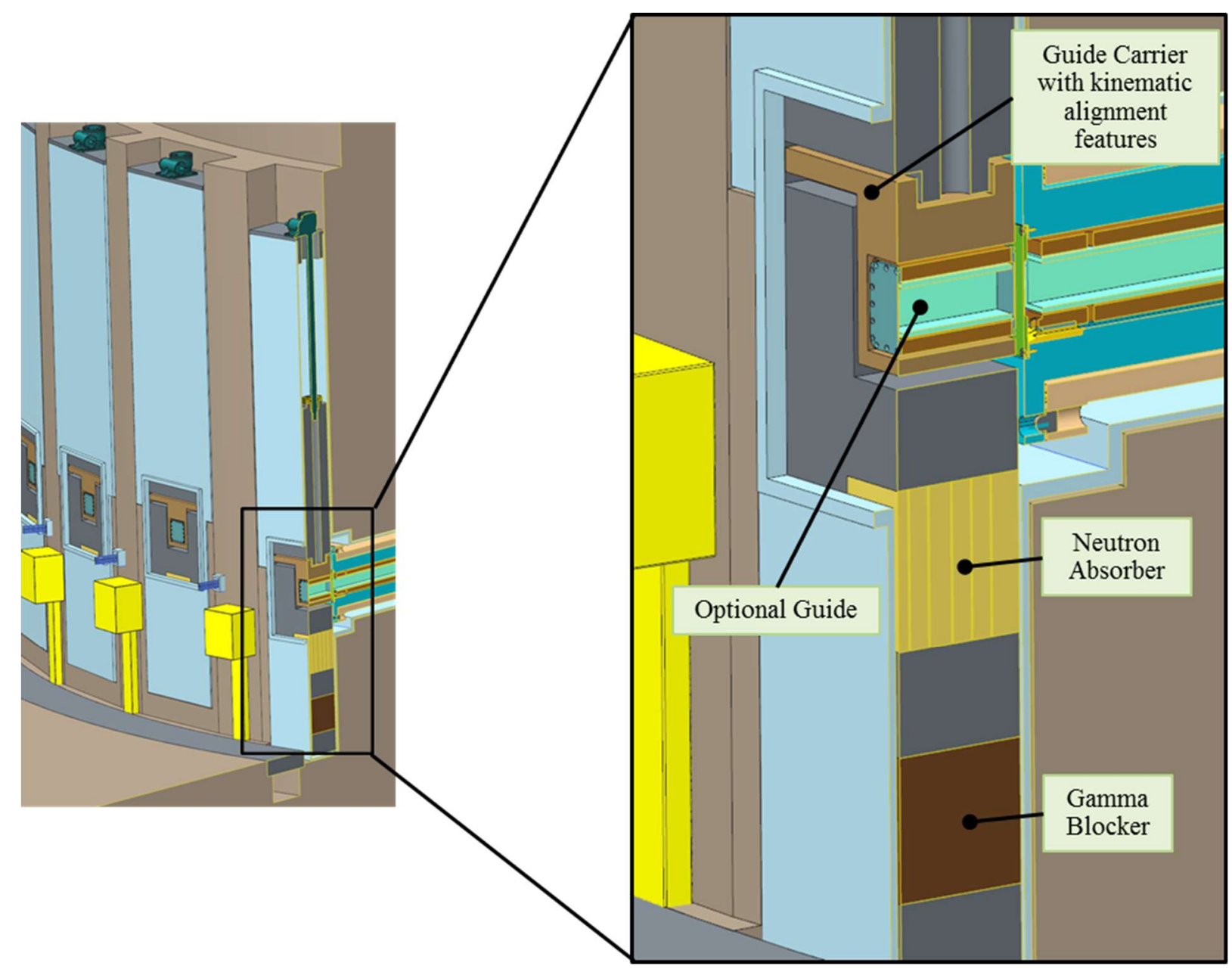

Figure 5.54. Maintenance shutters.

For beam line operations, each instrument will provide some sort of operations shutter. For those beam lines with sufficient curvature, a relatively thin neutron absorber plate might suffice for stopping lowenergy neutrons since the high-energy neutrons will enter the bulk shielding along the beam line; for those straight beam lines that have direct moderator views, a heavy shutter will be required. Based on FTS experience, an estimated length of such a high-energy neutron-stopping shutter is $1 \mathrm{~m}$ tungsten. Personnel access to the sample area and to any failed beam line components during proton beam-on is provided only by the operations shutter; access to failed components upstream of the operations shutter will only be possible during facility shut downs. Figure 5.55 shows conceptual operations shutter designs based on existing FTS implementations; these concepts are currently assumed to be the STS standards and are applied universally throughout the beam line concepts until more detailed neutronics analyses are available. 

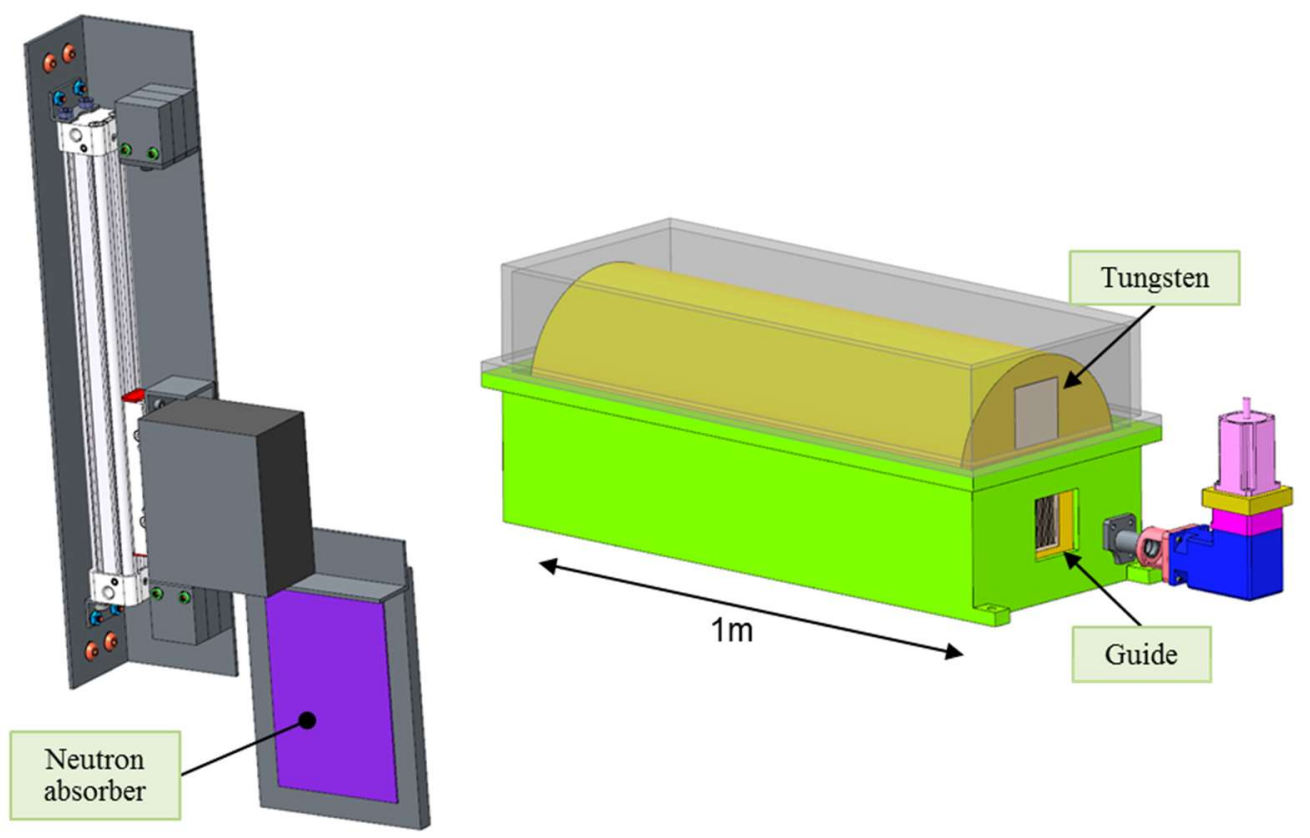

Figure 5.55. STS operating shutter concepts for low- and high-energy neutrons.

Since they are not space-constrained, long beam lines can generally locate high-energy, neutron-stopping shutters in a convenient location since the shutters do not typically affect instrument performance. However, some of the shorter instruments may have difficulty in allocating space for such shutters, especially since they should be in locations which allow vertical access for maintenance purposes. Locating operational shutters in the outer bunker wall of the short side of the facility, as shown in Figure 5.56 , is being considered. Slots in the bunker wall concrete would be required for vertical access, but building structural issues remain to be considered. In addition, the possible radiation levels within the bunker due to the close proximity of the most activated end of the shutter have yet to be considered in detail; however, it is assumed that local shielding can be implemented which still allows personnel access to the bunker.

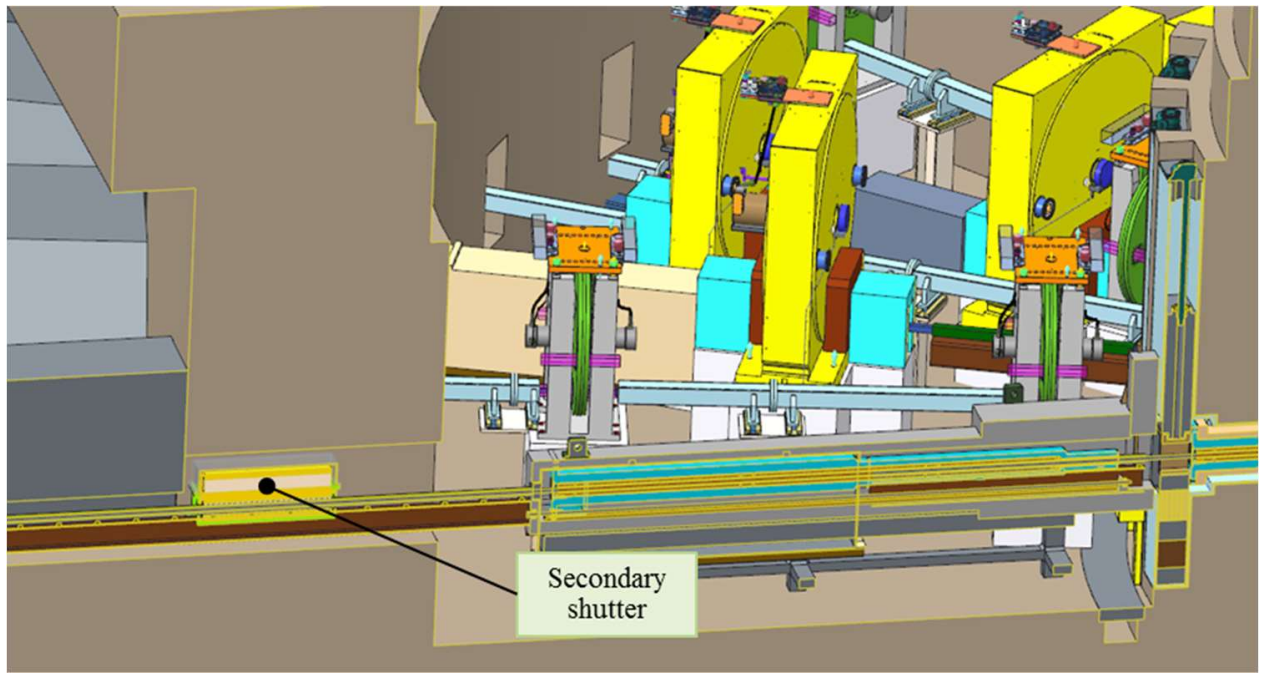

Figure 5.56. Operating shutter within the outer bunker wall. 


\subsubsection{Choppers}

Years of experience and development gained in producing and operating choppers in the FTS will be incorporated into the STS chopper designs. It is intended that the choppers be standardized to the maximum extent possible, especially for the commonly used $\mathrm{T} 0$ and disk choppers, to reduce design and fabrication costs as well as the number of spare parts.

\subsubsection{STS Chopper Requirements}

As the space requirements for the choppers contribute to the design and layout of the individual beam lines, it is important to have realistic estimates of their anticipated sizes, which are directly related to the neutron beam cross sections for given beam conditions. A fundamental requirement has been placed on the STS chopper design that the edges of the STS neutron pulse exiting a chopper have the same rise and fall time of the FTS neutron pulse exiting a chopper, where chopper speed equals source frequency. This definition of chopper speed and diameter also help to maximize the number of locations that a T0 chopper can be placed in the beam lines.

The current STS suite of moderators include square views of $3 \mathrm{~cm}, 5 \mathrm{~cm}$, and $7 \mathrm{~cm}$ along with a rectangular view $3 \mathrm{~cm}$ high by $6 \mathrm{~cm}$ wide. For the previously proposed $10 \mathrm{~Hz}$ STS source frequency and a $7 \mathrm{~cm}$ moderator view, the required chopper diameter is $1.8 \mathrm{~m}$ as compared to an FTS chopper diameter of $0.58 \mathrm{~m}$. The current $15 \mathrm{~Hz}$ STS source frequency reduces the worst-case chopper diameter to $1.25 \mathrm{~m}$. Figure 5.57 shows the relative sizes of $10 \mathrm{~Hz}$ and $15 \mathrm{~Hz}$ STS choppers. It shows that chopper disks could be as large as 1.8 meters in diameter to meet the needs of the STS at $10 \mathrm{~Hz}$. Also shown are smaller choppers with diameters of $1.25 \mathrm{~m}$ and $0.7 \mathrm{~m}$ which can meet the STS chopper requirements at $15 \mathrm{~Hz}$.

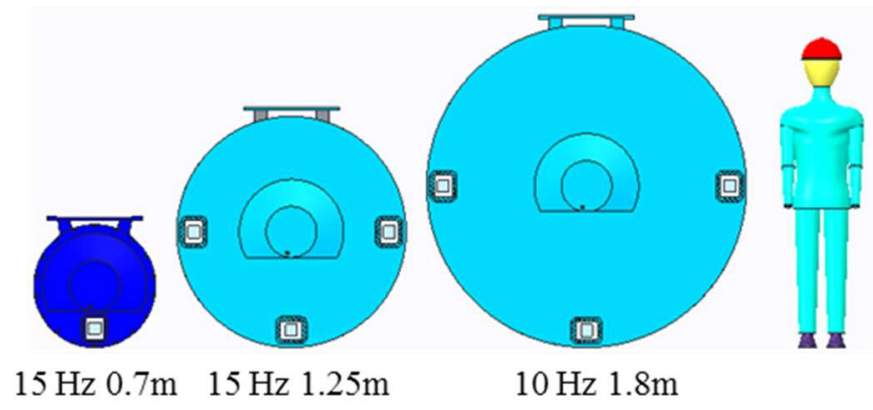

Figure 5.57. Comparison of $10 \mathrm{~Hz}$ and $15 \mathrm{~Hz}$ chopper diameters.

Such a reduction in size provides significant benefits in the STS beam line designs. Figure 5.58 shows comparative bunker views with $10 \mathrm{~Hz}$ and $15 \mathrm{~Hz} \mathrm{~T}_{0}$ choppers. There is significantly more bunker space available in a $15 \mathrm{~Hz}$ facility, and this will result in less impact between adjacent beam lines when selecting radial chopper placement within the bunker. Having smaller diameter choppers will also have positive effects on the widths of the $40 \mathrm{M}$ and trench beam lines, since chopper shielding is typically a size driver. 

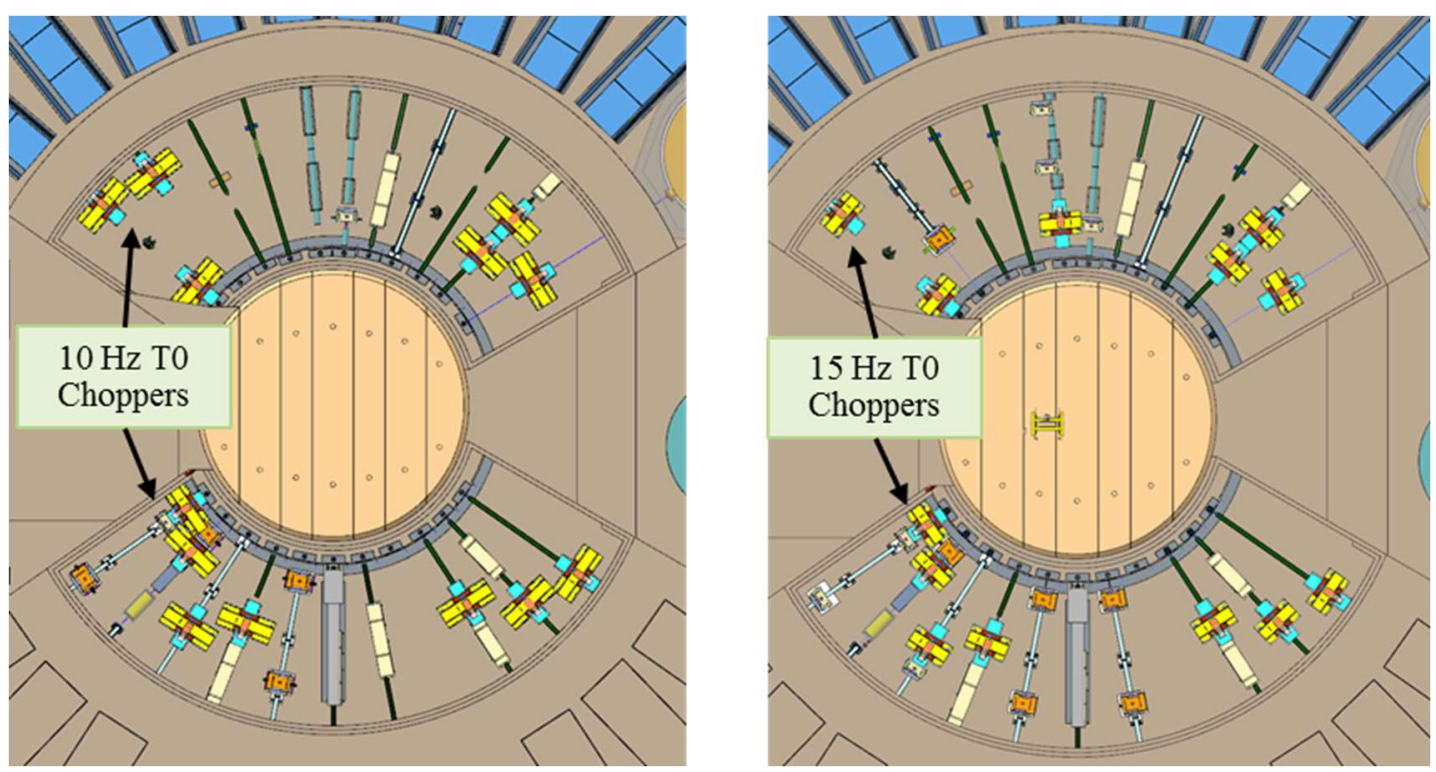

Figure 5.58. STS bunker views showing impact of source frequency on chopper size.

But even with the reduced diameters of the $15 \mathrm{~Hz}$ STS choppers, they are still twice the diameter of the FTS choppers, and when considering T0 choppers of this size for the STS, they can only be located within the bunker as shown in Figure 5.59 if other facility design goals, such as personnel access to the instrument buildings with sample environment equipment, are to be met. Smaller diameter T0 choppers will be available for use outside the bunker, but they will likely be double-rotor configurations.

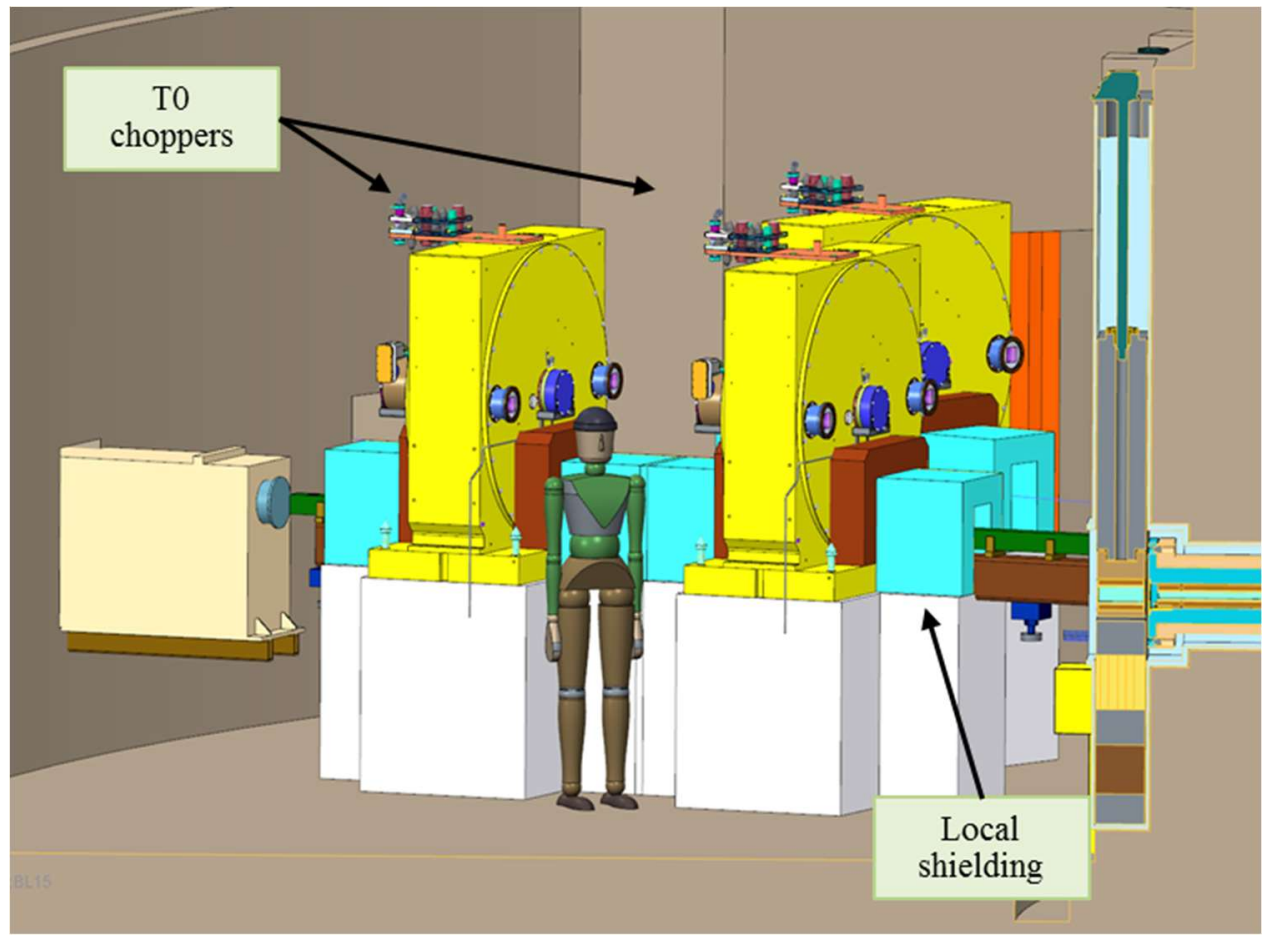

Figure 5.59. Close spacing of $T_{0}$ choppers within the STS bunker. 
Design concepts for many types of choppers expected to be used in the STS have been developed based on existing FTS designs. These concepts, shown in Figure 5.60, are primarily for accurate space claim within the STS design and will be updated as the overall design evolves.

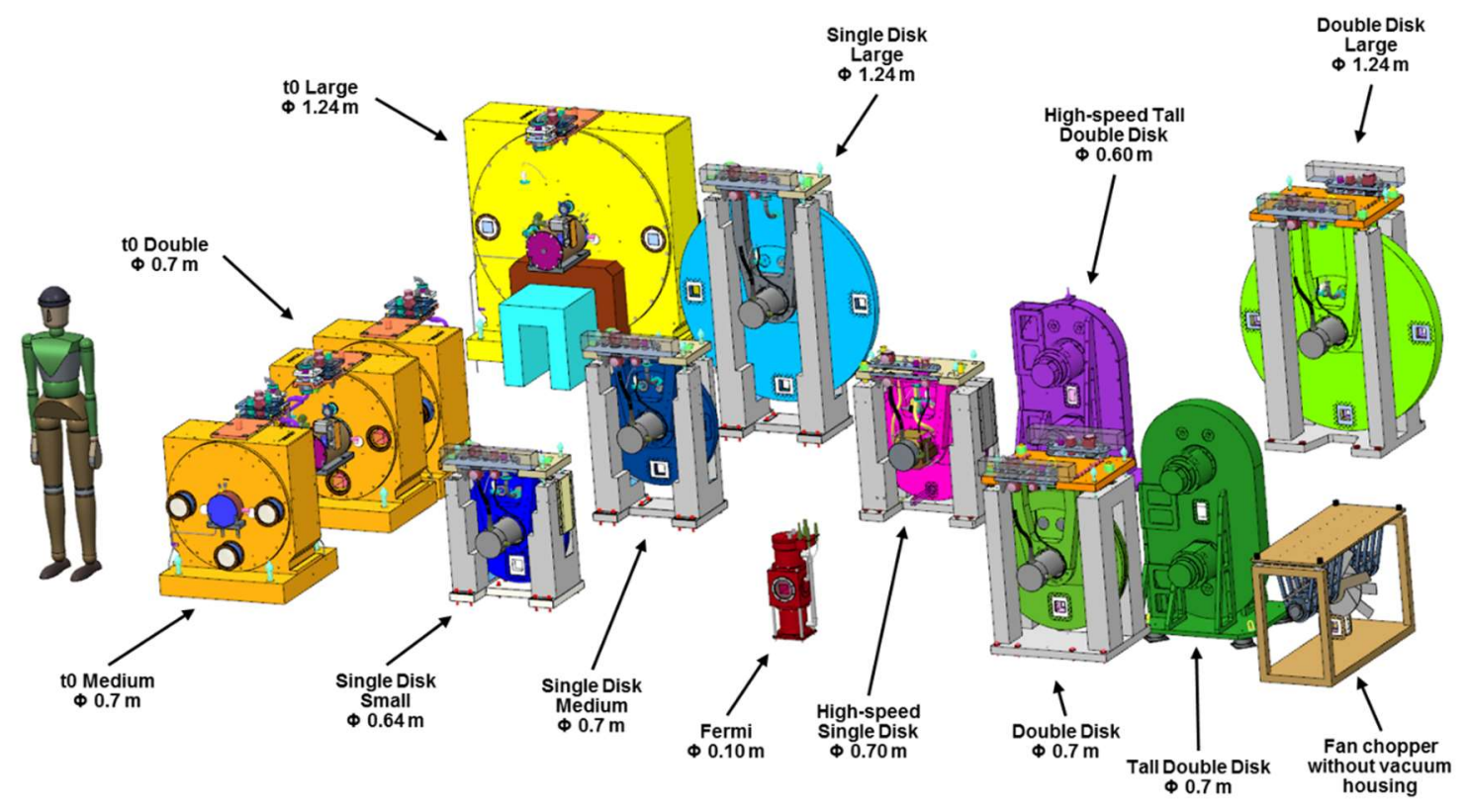

Figure 5.60. STS $10 \mathrm{~Hz}$ chopper library.

\subsubsection{Chopper Technology Development}

Many of the currently proposed STS instruments anticipate neutron optics which require higher stability and tighter alignment than was required for FTS instruments. Some instruments will use elliptical guides, while others will rely upon a focusing mirrors. With some beam lines approaching $100 \mathrm{~m}$ in length, stability of these optics will be critical to the performance of the instruments. Considering that in many instances rotating choppers will be near these sensitive optical components, chopper induced vibration is of utmost concern. All equipment rotating on mechanical bearings develop some amount of vibration that gets transferred to neighboring equipment. The transfer of vibration from the source to the receiver can be reduced, but the most effective means of reducing vibration transfer is to isolate the source.

Vibrations can be minimized by a combination of reducing design tolerances of parts, obtaining tighter fits during assembly, performing a higher-grade balance level of the rotating equipment, and using vibration dampers. Although the first three methods can reduce vibration, some amount is still transferred to the mounting structure and other equipment. In the case of vibration dampers, less vibration is transferred, but the equipment itself is left to vibrate, shortening its life expectancy significantly. The massive size of the STS T0 choppers multiplies the problem, because a heavier rotating mass equates to larger vibrations transferred into the mounting structure. Magnetic bearings can be used to isolate the source vibrations from reaching the mounting structure.

Magnetic bearings are already in use in most of the FTS choppers (most of the disk choppers, all the Fermi choppers, and all the Fermi-style T0 choppers.) They are also used in industry for equipment that is not only smaller, but also much larger than the STS T0 choppers. All the FTS blade-style T0 choppers use mechanical bearings. There are no magnetic bearing, blade-style T0 choppers which currently exist, let alone with the diameters and mass required by the STS. However, all that is needed for the development of suitable STS T0 choppers is the design of correctly sized magnetic bearings, a specification for a motor 
to meet the chopper phasing requirements, and integration with the rotor and housing design. Chopper manufacturers have already designed more powerful magnetic bearing spindles to control the higher forces associated with disks of future, large-disk choppers. The motors in the magnetic bearing spindles required updating to provide higher torque motors to accelerate the large diameter disks to speed in a reasonable amount of time and have sufficient power to properly maintain chopper phasing. In addition, the control systems have been modernized with 1) higher speed processors to provide faster control of the system while also offering greater functionality with even more parameters, 2) hardware to utilize current communication technology, and 3) a host of firmware and software upgrades to better diagnose and manage control issues of the system. After a preliminary investigation, it's been determined that one of these new control systems has enough power to control a STS magnetic bearing T0 chopper. In addition, the manufacturers are also implementing lessons learned from previous systems to provide faster, more repeatable tuning techniques, which, with one manufacturer, allows the tuned system to meet Parts 1-3 of ISO 14839, Vibration of Rotating Machinery Equipped with Active Magnetic Bearing Systems.

Although none of the chopper manufacturers have ever built a chopper with a disk diameter of 1.8 meters, one manufacturer is currently considering options for such a chopper. Current thinking is that aluminum disks won't be flat enough, or will be too heavy and expensive once they have been manufactured with sufficient rigidity and flatness. There is also a belief that a standard carbon fiber disk would not be flat at these very large diameters, as they release tension and warp when neutron beam apertures are machined into them, even at diameters of 0.7 meters. One manufacturer is considering a hybrid material of carbon fiber and honeycomb aluminum that is used to support antennas on space satellites. This material is very lightweight and should have the stability required to maintain flatness. Unfortunately, this is still conceptual and needs to be proven by manufacturing a disk of sufficient diameter.

Investment in an existing technology that has already proven that it can easily eliminate vibrations will provide a significant cost savings over the life of the facility. Although the potential magnitude of chopper vibrations and the forces imposed upon the mounting structure have been scaled from actual, measured vibrations of existing T0 choppers, there is no way to anticipate how large of an impact these transferred vibrations will have on the focusing equipment without characterizing an assembled system that accurately represents the intended installation structure. The use of magnetic bearing T0 choppers would eliminate the source of the problem rather than trying to reduce its effects after its development. Using existing magnetic bearing technology with its documented results is the most cost-effective way to eliminate the possible impact of T0 chopper vibration on STS instrument performance, and this technology is being considered for implementation in the STS T0 choppers.

\subsubsection{Beam Line Shielding}

A preliminary McStas calculation based on a $15 \mathrm{~Hz}$ operating frequency and $700 \mathrm{~kW}$ beam power has been performed to estimate the shielding thicknesses required for the STS beam lines. In this calculation, constant cross section guides matching their moderator-viewed face sizes were assumed, both for straight beam lines and curved beam lines with a radius of $2000 \mathrm{~m}$. The results of this calculation are shown in Figure 5.61, where the curves give the thickness of both regular- and high-density concrete (RDC, HDC) needed to meet the STS radiation shielding requirements. 

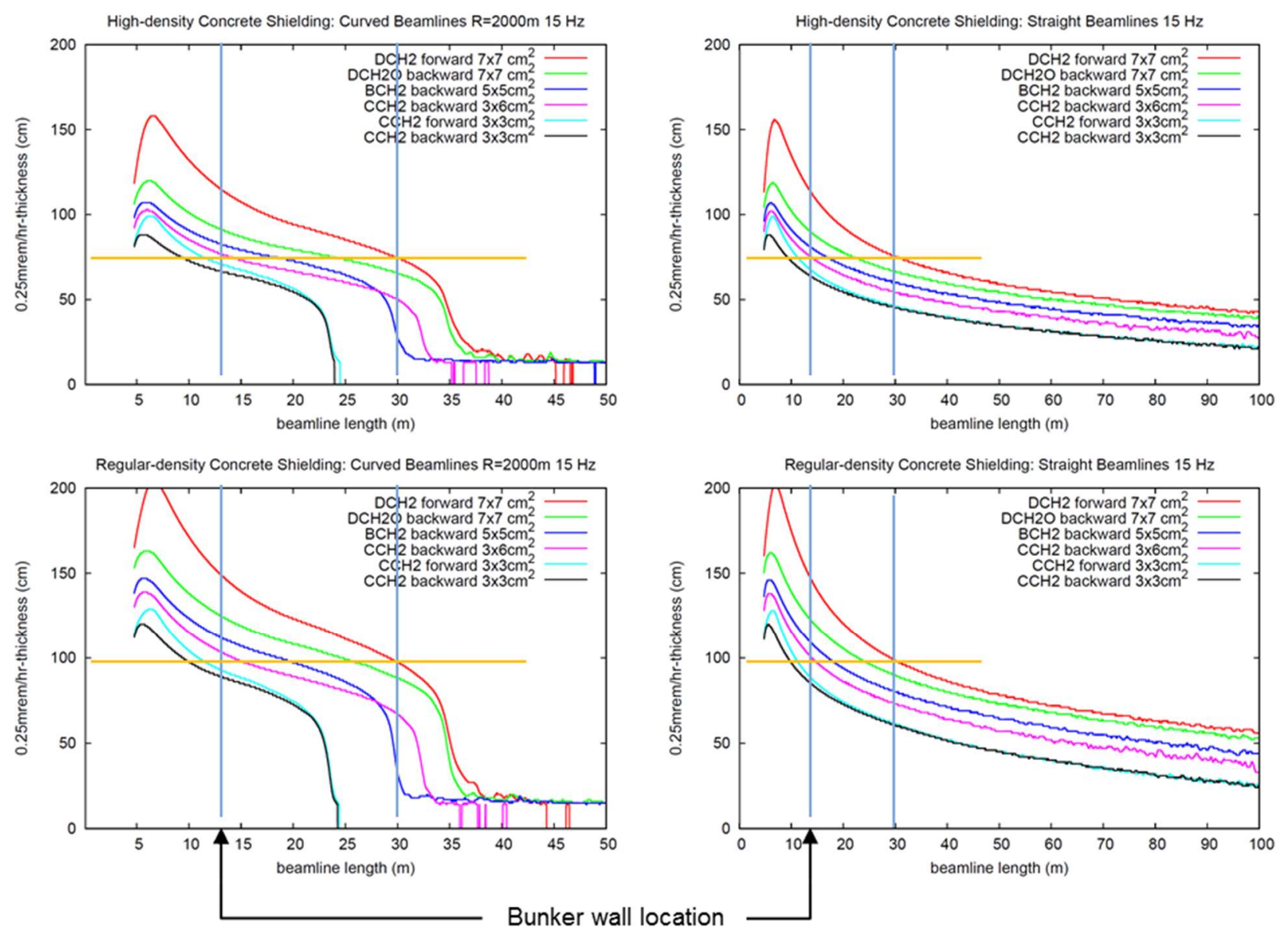

Figure 5.61. Estimated shielding thicknesses along the STS beam lines.

From the plots in Figure 5.61, it is seen that the $7 \times 7 \mathrm{~cm}$ decoupled, hydrogen, forward-facing moderator is the worst case and is being used as the baseline for the STS beam line shielding design. The calculation indicates that approximately $1 \mathrm{~m}$ of RDC is required around the beam guides out to a radial distance of approximately 30 meters. This is shown graphically for the STS long beam lines in Figure 5.62. It can also be seen that for curved beam lines, the shielding requirements drop significantly after about 35 meters. And as noted in Section 5.2, the outer bunker walls and removable ceiling shield blocks will be 2meter-thick HDC to account for multiple radiation sources. The results shown in the plots of Figure 5.61 were the driver for the neutron guide bunker shielding design. 


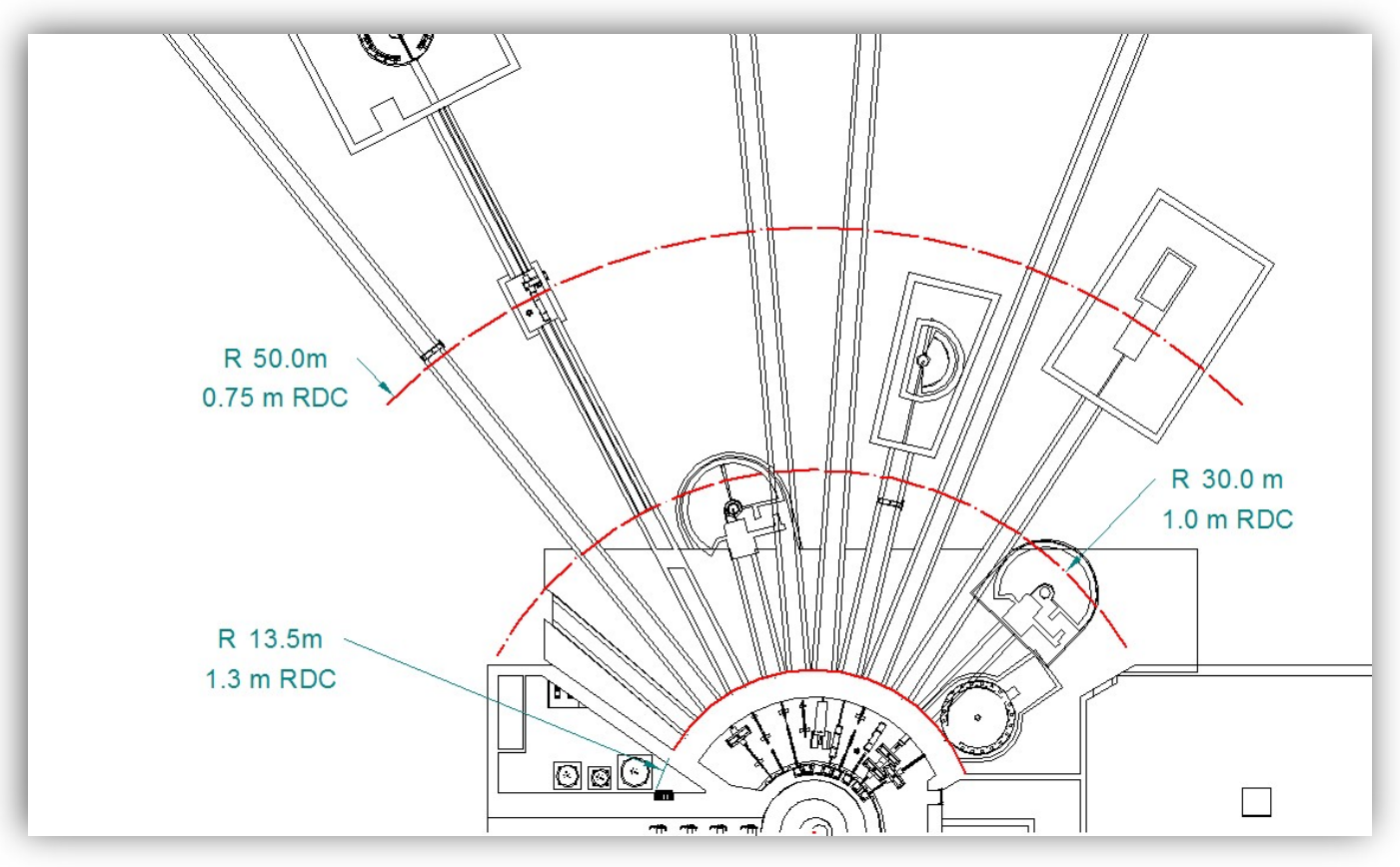

Figure 5.62. Beam line shielding extent.

\subsubsection{Long Beam Line Trenches}

A general description of the long beam line trenches was provided in Section 5.2. One of the benefits and major criteria in selecting trenches as the long beam line concept is the relative ease of access and movement of sample environments to the instrument buildings. The topology of the STS site makes instrument building access from only the exterior inconvenient at best, and at worst would significantly reduce scientific operating efficiency and possibly jeopardize sample and sample equipment integrity during transport. Figure 5.63 shows the long instrument side of the STS and the personnel \& equipment pathways from the target facility out to each instrument building. The trench area just outside the bunker is open to the high bay and is accessible with the 50T overhead crane. Mobile 10T (capacity driven by weight of choppers and trench shield blocks) gantry hoists can also access this space as well as the entire length of the trenches. These hoists will be used to install trench shield covers in addition to the beam line choppers and other optical components. For some of the longer components, two mobile hoists may be needed, but they can be moved from one beam line to another as required. 


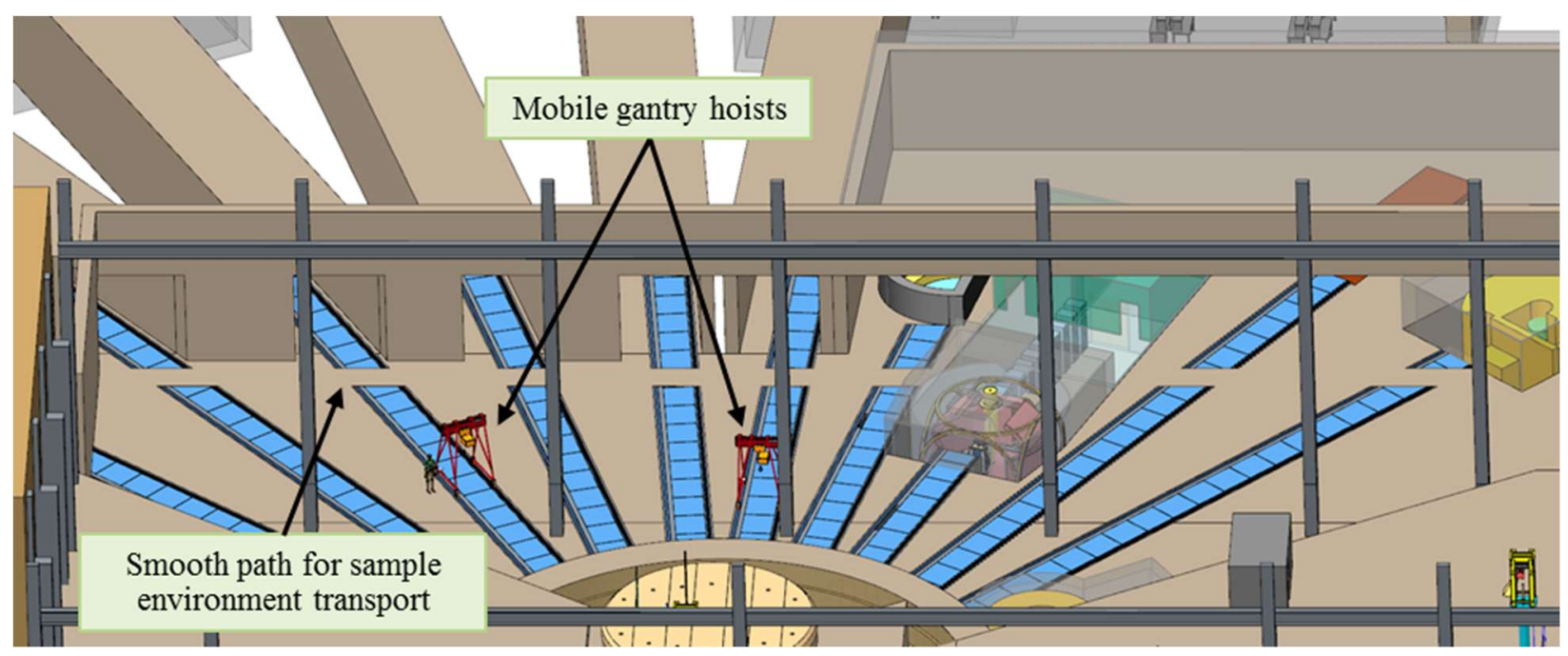

Figure 5.63. Long instruments in the STS.

Compared to long above ground beam lines such as the BASIS instrument in FTS, the cross section of the trench enclosures outside the Target Building is relatively compact since the shielding is entirely below the floor. The BASIS beam line, shown in Figure 5.64 looking towards the detector, incorporates a fullwidth mobile gantry hoist for lifting the shield blocks but also requires enough width for a fork truck since the hoist does not incorporate a powered base and cannot be used for transporting items along the beam line. Not shown in the figure is the significant increase in the shielding stack size as the beam guides get closer to the moderator.

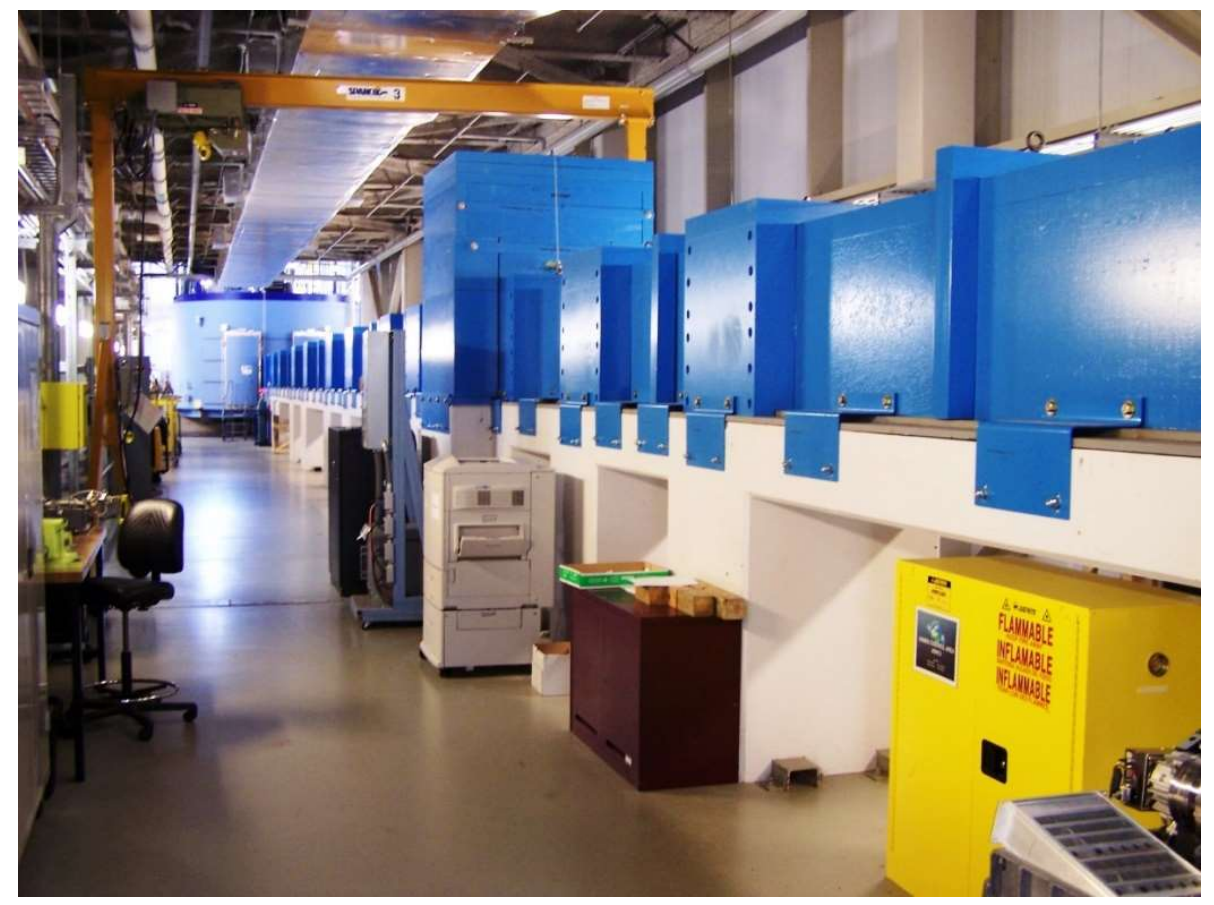

Figure 5.64. FTS BASIS beam line.

Figure 5.65 shows the conceptual layout of the STS trench enclosures. It provides enough space beside the trench to accommodate movement of personnel, sample environments, liquid nitrogen dewars, and other portable equipment out to the instrument buildings using pallet jacks if necessary, but it is not sized 
for fork trucks. Vertically, the enclosure must accommodate the mobile gantry hoists capable of removing choppers along with HVAC and other associated utilities. Chopper controllers, vacuum pumps, electronics racks and other related equipment will be stored within the trench enclosures, either between the structural columns or on the trench covers themselves. Dog-leg conduits will be embedded within the trench walls to allow utilities, instrumentation, and vacuum services to be distributed along the trench as required.

It is understood that the trench environment must be closely controlled to minimize temperature or humidity changes along the beam lines. In addition, while water proofing of the trench walls must be excellent, provisions for handling water ingress must still be incorporated into the design.

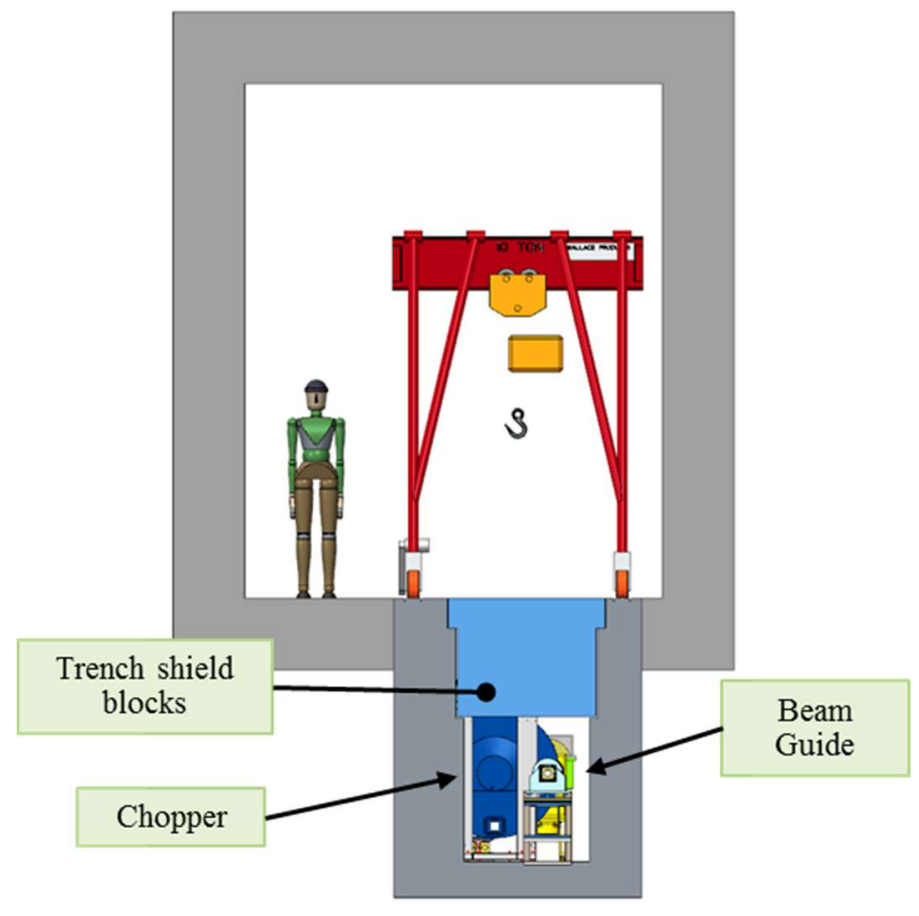

Figure 5.65. Typical trench cross section.

The basic dimensions of the trenches are driven by the amount of removable shielding above the beam line components and providing adequate space for personnel to install and maintain the beam line components. Figure 5.66 shows the beam line components located within a trench and the space allotted for personnel access. It is noted that the trenches are not symmetric about the beam line and that the beam guides are biased within the trenches such that personnel access is from a single direction. Trench depths differ by $30 \mathrm{~cm}$ for lower- and upper-moderator beam lines. Within the target building, trenches will likely be formed when the instrument floor is poured; outside the target building, the trench wall thickness and construction will be determined by structural and/or ground water shielding requirements. As all the neutron beam lines will be supported by driven piles, the trenches must be self-supporting between the piles. 


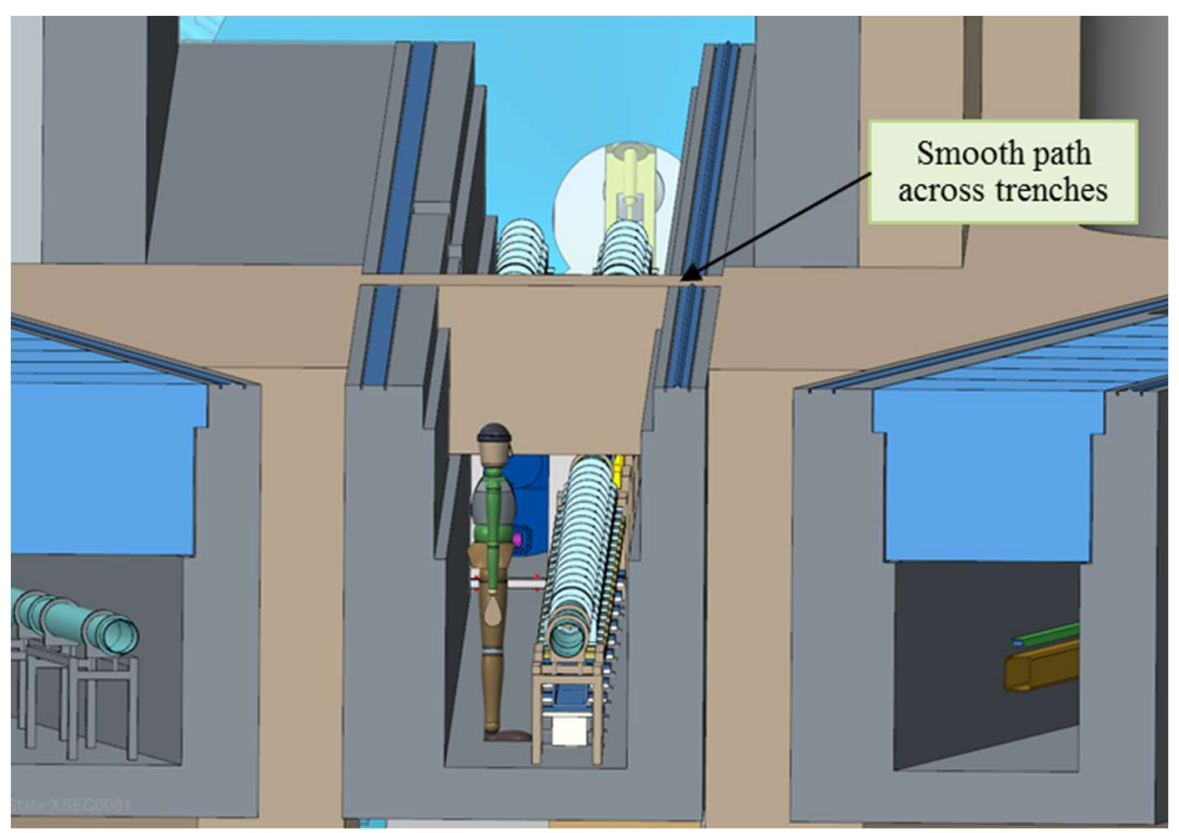

Figure 5.66. Personnel access of beam line components within a trench.

To a large extent, neutron beam line geometries are driven by the size of the choppers; trenches must be sized to accommodate them, and the overall width of the short lines is driven by their shielding requirements. Figure 5.67 shows a notch within the trench wall required to accommodate a chopper. It is noted that one of the side beam windows is used in the trenches to reduce the overall trench depth; this has the effect of biasing the chopper's location away from the beam line but eliminates the need for a mounting riser. The bottom chopper beam window is predominately used when choppers are positioned in the bunkers or in a short beam line on the $40 \mathrm{M}$ side of the facility.

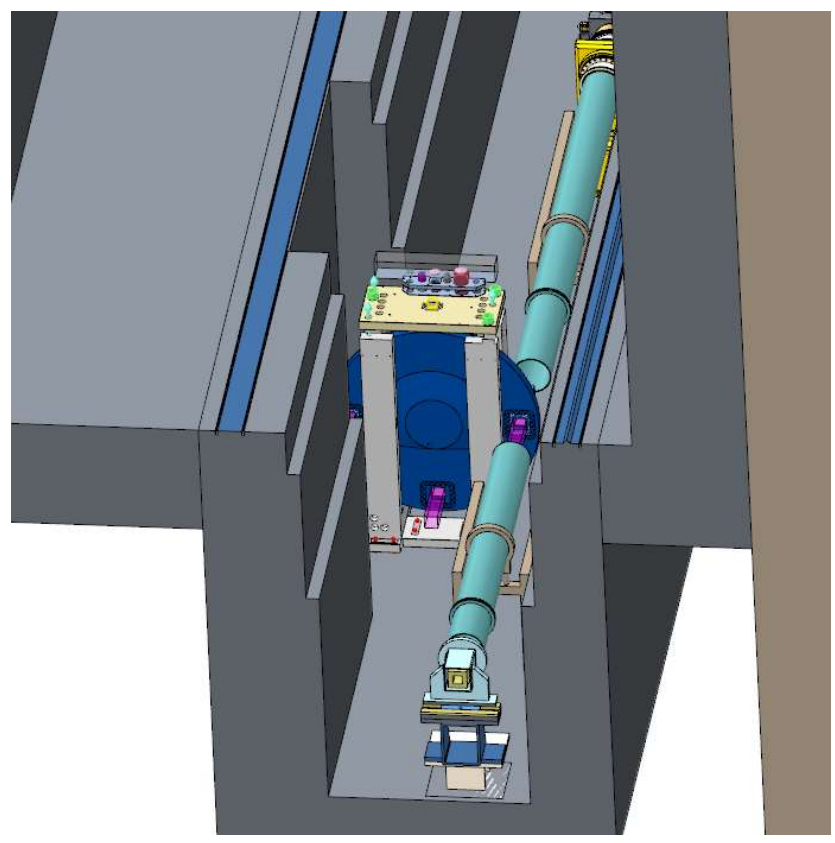

Figure 5.67. Trench cutout for chopper. 
At this early stage of conceptual development, all the trenches are assumed to be identical, but customizations may be implemented as the instrument and beam line concepts evolve.

\subsubsection{Component Alignment}

Accurate alignment of all the target station and beam line components is a basic requirement of the compact neutron source. Four separate alignments are involved in determining the necessary precision to provide optimum physics performance. Figure 5.68 shows the spatial relationship between the target disk and the moderators and clarifies the alignment requirements.

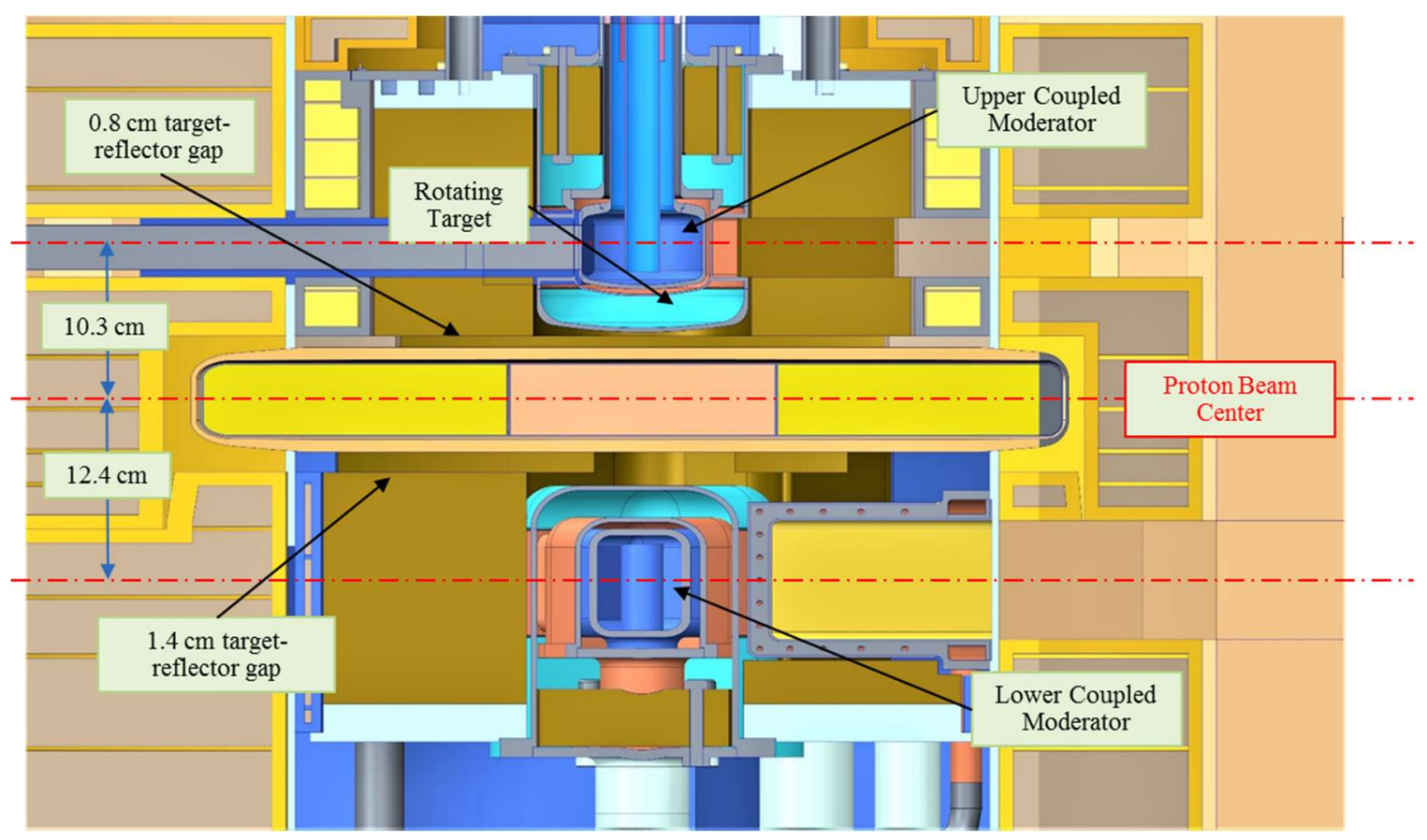

Figure 5.68. Geometric relationship between target and moderators (proton beam enters page).

\subsubsection{Proton beam to target}

To achieve the maximum neutron flux from the target, the excess neutron absorbing area on the top and bottom of the target must be held to a minimum. Based on the performance of the FTS accelerator, the beam centroid will be held within a margin of $\pm 0.5 \mathrm{~mm}$ which drives the target block height of $5 \mathrm{~cm}$. To support this capability, STS provides three systems to maintain the proton beam in the center of the target. The direct viewing periscope with the ability to optically locate the position of the target center within $\pm 0.2 \mathrm{~mm}$ and the position of the proton beam centroid on the target within $\pm 0.5 \mathrm{~mm}$ will provide the most accurate alignment guidance. Based on the performance in FTS, the STS wire harp and halo monitor will back-up the optical system by tracking the beam centroid to less than $\pm 4.0 \mathrm{~mm}$ and beam drift to less than $\pm 0.5 \mathrm{~mm}$.

\subsubsection{Moderator to target}

Neutron production is improved by locating the moderators as close to the target as practical. The STS design minimizes this gap by addressing several potential mechanical and fabrication issues. Currently, the design calls for an $8 \mathrm{~mm}$ upper gap and a $14 \mathrm{~mm}$ lower gap. The lower clearance provides for thermal expansion of the target disk because the 4-meter axle will be exposed to a beam-off, maximum cooling water temperature of $70^{\circ} \mathrm{C}$ above the normal operating temperature of $30^{\circ} \mathrm{C}$. A similar thermal issue in 
the moderator/reflector assembly is addressed by positioning the mounting frame on fixed points near the target center (Figure 5.69) rather than at the top of the core vessel. Additional thermal clearance is not required since the moderator/reflector frame movement will track with the target disk movement. A target disk and axle manufacturing tolerance is expected to be less than $\pm 1 \mathrm{~mm}$ based on the full-scale rotating target mockup and input from a major US component vendor. Alignment of replacement target and moderator/reflector assemblies will be aided by pre-installation checkout in the target monolith mockup located in the target building.

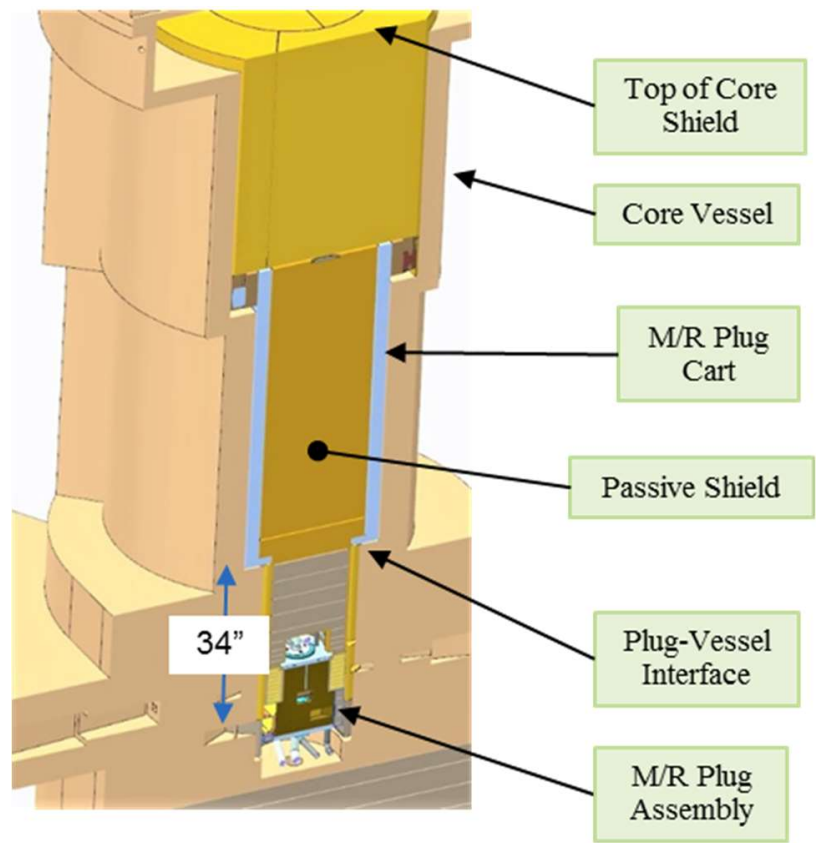

Figure 5.69. Moderator-reflector assembly installation interface.

\subsubsection{Moderator to neutron beam lines}

The 22 neutron beam lines are distributed among 3 moderators. Each beam line is assigned to a single moderator view in the facility design; thus, the beam ports do not allow a different moderator-beam line configuration during beam line installation. In addition, the beam ports will not be oversized to accommodate significantly larger moderators in the future. Each beam port will be fabricated to be vertically centered on its assigned moderator face during steady state operation, and the moderators will be vertically oversized so that the neutron production is not affected by assembly tolerances or small thermal movement of the moderators.

Figure 5.70 shows the STS vacuum vessel along with its beam ports and their associated nozzles. The nozzles will be installed on-site during facility construction after the vacuum vessel is set in position and the lower bulk shielding has been added, with the upper bulk shielding assembled afterwards so that the entire monolith weight will be in place before any alignment operations commence. The neutron beam optics must interface with the core vessel beam port flanges and their associated nozzles in this configuration. These large components significantly influence the ultimate location of the neutron beam optics, both the initially installed instruments as well as those installed in the future, so accurate measurement and mapping during fabrication and installation is critical. Any fabrication and installation errors within these components must be accounted for in the final installation and alignment of the optical systems. Input from a potential vendor of vessel and extensions has suggested that positional and angular tolerances on the mating vessel and extension faces are $\pm 0.013 \mathrm{~mm}$ and $\pm 0.002^{\circ}$. This leads to a 
requirement that the downstream end of the monolith Inserts must accommodate positional adjustability of $\pm 0.27 \mathrm{~mm}$ to allow the inserted guide modules to be located on the nominal beam centerlines. This adjustability will come in the form of shims that are installed during component installation.

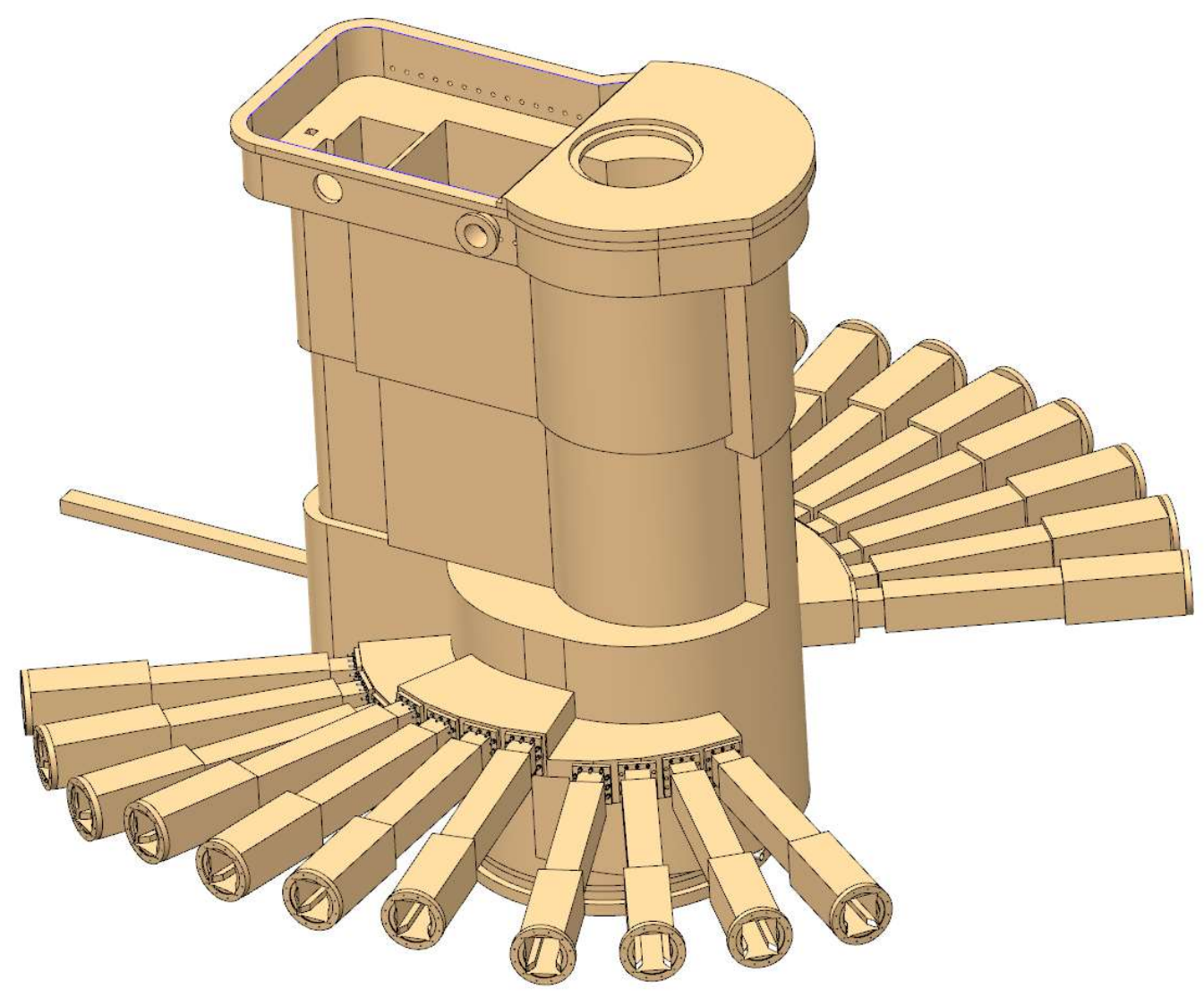

Figure 5.70. STS Core Vessel and Nozzles.

As shown in Figure 5.71, the monolith inserts will incorporate external alignment features which will position them within the nozzles and port flanges. The portion of the inserts upstream of the front shims are cantilevered about the bottom front shim and can pivot vertically and horizontally about that location as dictated by the rear shims. 


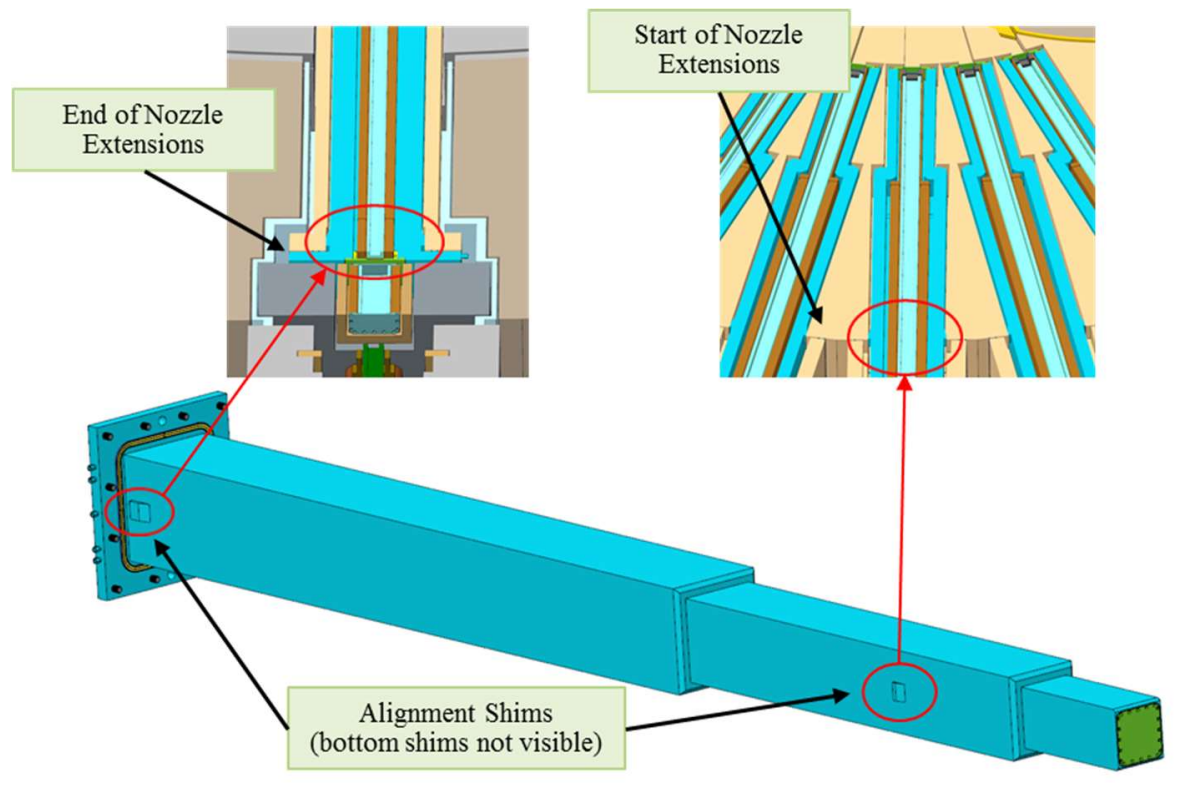

Figure 5.71. External alignment features of monolith inserts.

The inserts will also have internal alignment features that interface with mating features on the guide modules that are installed later. These internal features are conceptually shown in Figure 5.72; adjustments to the alignment of the guide modules are controlled with shims installed on the modules prior to installation. Because the guide modules may not be installed for several years after STS construction, the internal alignment surfaces of the inserts must be mapped and recorded prior to their installation. It is noted that because the monolith inserts and guide modules are designed for remote removal and installation, additional clearance of $6 \mathrm{~mm}$ around these components is assumed, so the internal adjustability features should not impose any additional space requirements within the nozzles or inserts.
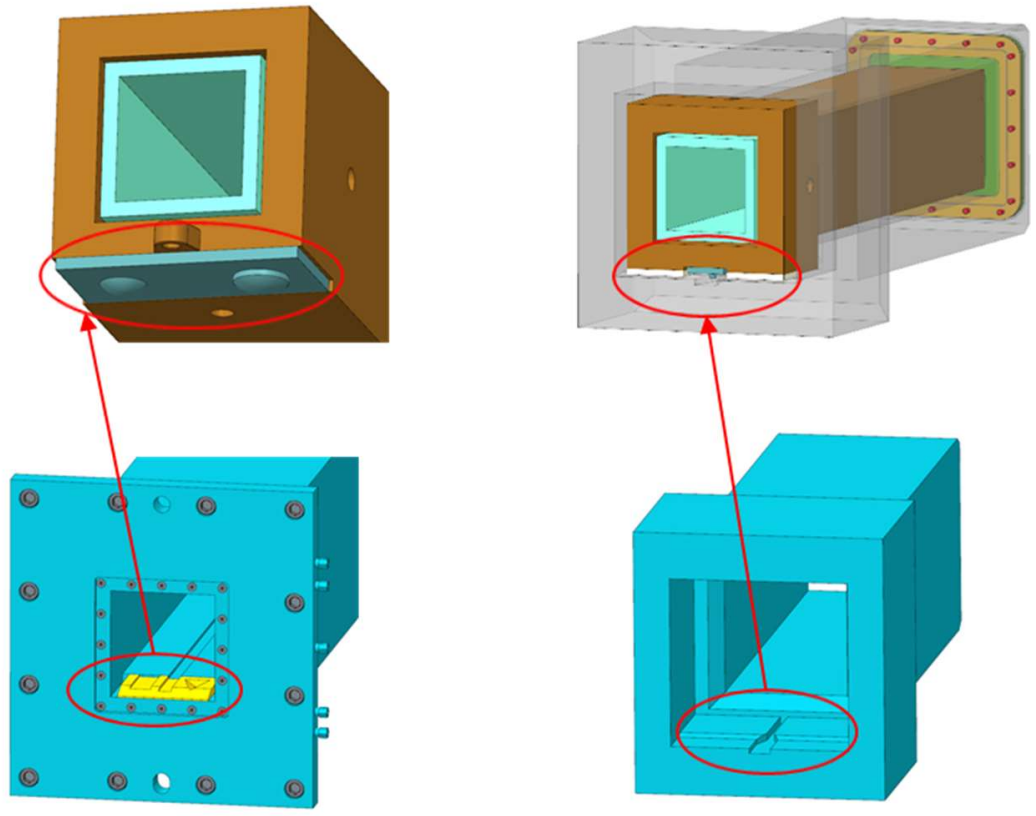

Figure 5.72. Internal kinematic alignment features of the monolith inserts. 


\subsubsection{Neutron beam optics}

The optical components used by STS instruments must be aligned to much greater precision than the neutron guide optics used by FTS instruments. There are several reasons for this, primarily due to the increased brightness of the STS moderators, where the use of more sophisticated optical components can achieve even higher instrument performance than one would expect from the additional brightness alone. The development follows the natural progression from neutron beam transport (neutron guide) to focusing optics (elliptical and converging guides) to imaging optics (elliptical, hyperbolic, parabolic, and logarithmic mirrors) and parallels a very similar development path in x-ray optics. The use of imaging optics on neutron scattering instruments entails large mirrors, several meters in length, separated by tens of meters. This poses some challenges:

- In many cases, the mirrors are much too large to be fabricated as a single piece but must be a composite mirror assembled from several individual mirrors. The assembly must be aligned well enough to obtain the required figure of the composite mirror. The acceptable figure error in the composite mirror will depend on the particular demands of the optical system of which it is a part, but in nearly all cases it will exceed what has been required for past installations.

- Once the mirror assemblies are aligned, they must be placed on the beamline at the correct orientation and location. The allowable tolerance for this alignment also exceeds what has previously been required for neutron scattering beamlines.

Fortunately, SNS has some experience on which to draw for both cases. The allowable figure error of a mirror assembly is not drastically different to what is required for the nested Kirkpatrick-Baez mirrors which have been constructed and tested in the past [19]. This work can be extended to produce mirror assemblies of the size required for STS beamlines. And for the placement and orientation of the mirror assemblies on the beamline, the STS requirements can be estimated from SNS' experience installing and aligning the mirrors on the IMAGINE beamline at HFIR. Here, two elliptical mirrors were used in series to image the entrance slit onto the sample. Each of the mirrors was about $70 \mathrm{~cm}$ in length, and the distance from the entrance slit to the first mirror was greater than 10 meters. It was observed that translation less than $0.1 \mathrm{~mm}$ and rotation less than 0.01 degrees had no measurable effect on the focal image at the sample position. These deviations simply scale with the length of the mirror and the distance between mirrors. So, for STS instruments where the length of the mirrors and the distance between are not expected to exceed a factor of 10 , then the alignment tolerances will scale to $0.01 \mathrm{~mm}$ for position and 0.001 degrees for rotation. A system which can meet such alignment requirements is presented here.

For these imaging optics, some effort has been spent developing a concept for alignment and remote adjustability of these mirrors. Some of these mirrors will be several meters long and will be formed from glass segments on the order of $500 \mathrm{~mm}$ in length as is currently done for curved guides. The current concept assumes these segments will be housed within vacuum housings and that the group of housings will be mounted onto a stable strongback such as a granite or steel beam using manually adjustable kinematic mounts as shown in Figure 5.73 to account for deflections within the strongback. 


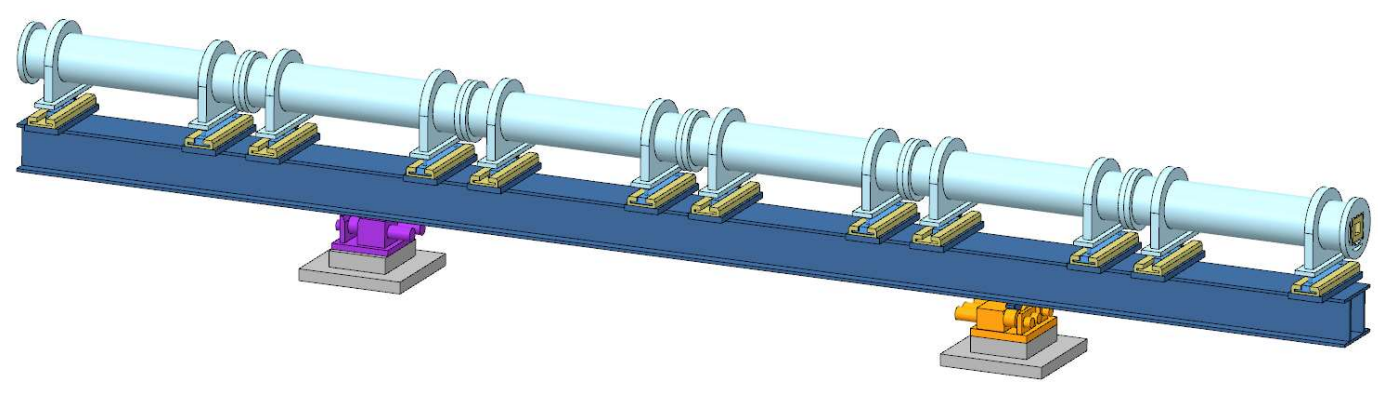

Figure 5.73. STS MENUS focusing mirror assembly on remotely actuated precision movers.

The entire mirror shown in Figure 5.73 is 6 meters in length with 1-meter-long segments. Assuming a granite strongback, such a system would likely weigh 1-2 tons. It is expected that several STS instruments will require such mirror systems, so a standard method of incorporating these mirrors is needed. Figure 5.74 shows the EWALD beam line concept near its detector and includes the second of two elliptical imaging mirrors on a remotely adjustable support system.

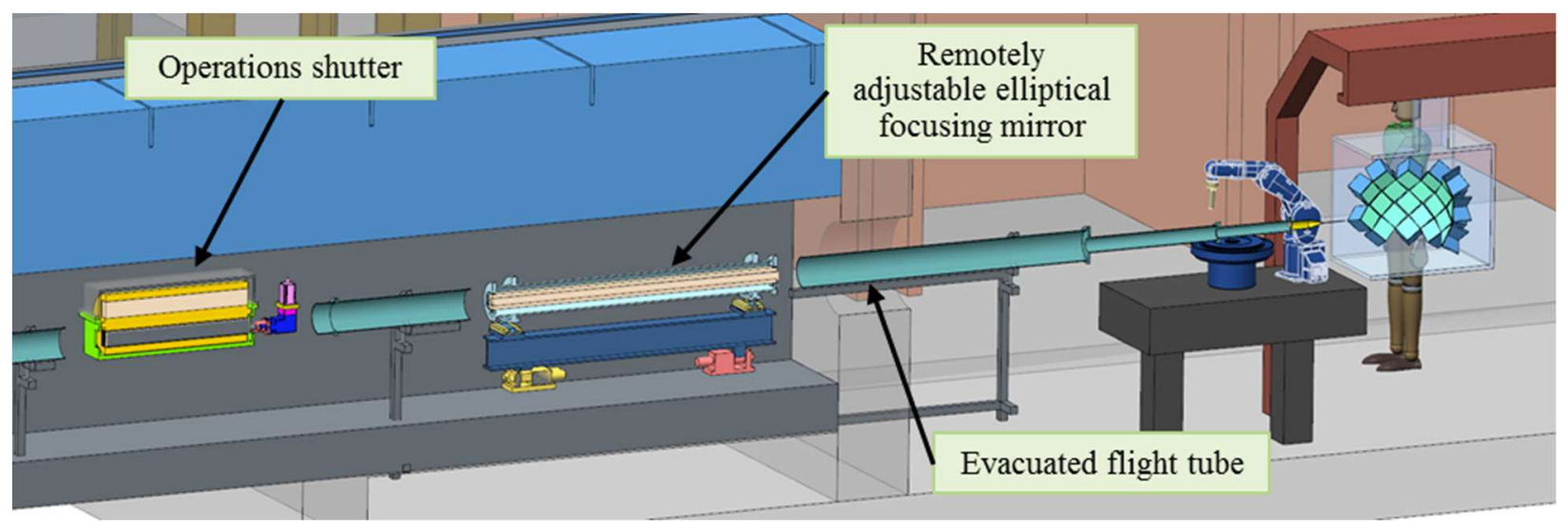

Figure 5.74. Final focusing mirror within STS EWALD instrument concept.

For the external alignment of the mirror to the beam line, typical guide installation methods of fixed supports and manually adjusted mounts using standard survey and alignment techniques, which may be accurate to within 50-100 microns, are not likely to be sufficient to ensure that neutrons are focused on the sample, especially since many STS beam lines which desire to incorporate these nested mirror systems may be up to 100 meters in length. So, a method of remotely adjusting the entire mirror assembly real-time is proposed. Such a system would need to stably support weights of a few tons over many years but still provide micron-level position and angle adjustability. This is conceptually shown in Figure 5.73 as a two-point support system with motorized actuators.

Such a remote alignment concept is based on a magnet mover system developed for the Final Focus Test Beam at SLAC in the mid-1990s [20]. This system used eccentric cam rollers driven by high-gearreduction stepper motors to move $100 \mathrm{~kg}$ magnets over a range of a few millimeters with sub-micron precision. The basic mechanics of a 3 degree-of-freedom (DOF) mover and a 5 DOF mover suitable for STS focusing optics are shown in Figure 5.75. 


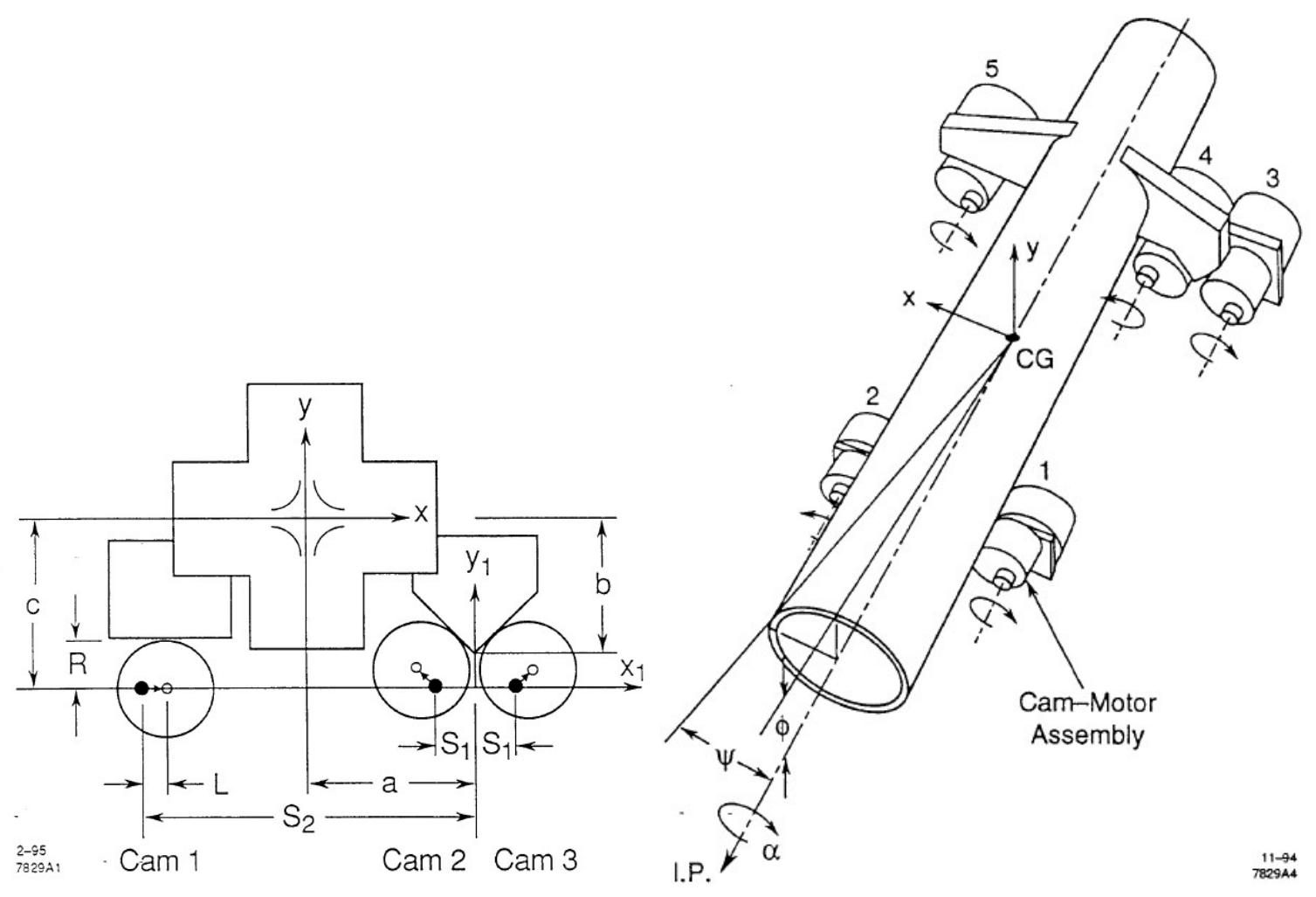

Figure 5.75. Images from SLAC-PUB-95-6132 showing the operating principle of 3- and 5-DOF eccentric roller movers.

Since the initial deployment at SLAC, other facilities have successfully implemented this high-precision mover system to support heavy magnet systems. As shown in Figure 5.76, examples include the storage ring at the Swiss Light Source (SLS), the LCLS Undulator Magnet support system at SLAC, and the ATF2 Final Focus Beam Line at the KEK High Energy Accelerator. No implementations of such mover systems within neutron scattering facilities are known, however, so it is likely that STS may be the first to consider such a system for neutron beam focusing optics. While the engineering of such a system will require some development effort and testing, implementation at STS should be straightforward given that such systems are operational in several scientific facilities.
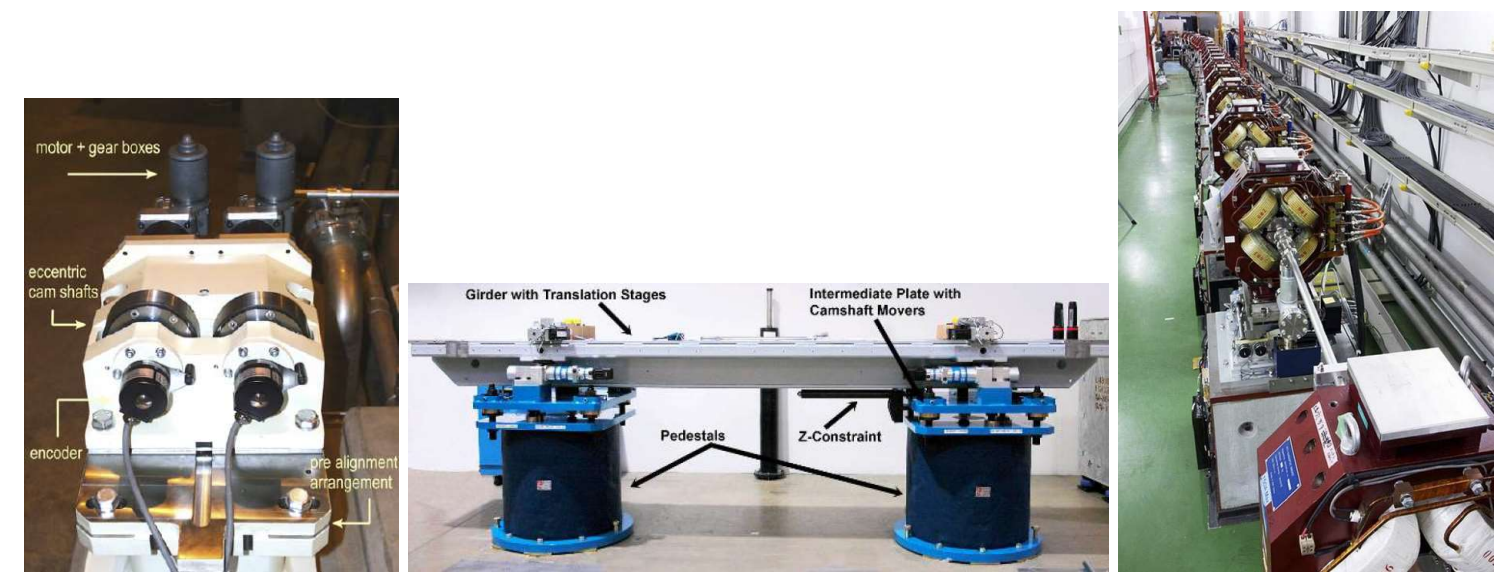

Figure 5.76. Examples of magnet mover implementations at SLS, SLAC, and KEK. 
It is understood that rigidity and long term stability of such structures is critical to their successful implementation and that environmental conditions must be tightly controlled. Experience with the HFIR IMAGINE beam line suggests such rigidity and stability are possible, but as the mirrors grow larger this becomes more of an issue that will be investigated through mockups. The ability to use real-time neutron beam scanning will negate the need to initially install focusing optics to micron-level precision.

\subsection{SERVICES AND OPERATIONS}

\subsubsection{Water Process and Cryogenic Systems}

The water process system matches the FTS system in configuration and equipment selection, simplifying maintenance activities and spare parts storage. The system has two primary loops: one for the target and proton beam window, and the other for the balance of plant. As with FTS, certain components such as the moderators will have special heat exchanger provisions to control the water temperature within approximately $1^{\circ} \mathrm{C}$. One significant difference is the addition of full flow filtration of the target return flow to limit contamination of the process system.

At this time, STS uses only light water with no provisions for heavy water. If this requirement changes an additional loop will be added.

The cryogenic systems also mirror the FTS system to simplify operations and maintenance. No significant changes in size, capacity or configuration are planned.

\subsubsection{Maintenance}

Maintenance issues are a very important factor in the design of the highly-activated components; consequently, lifetimes, handling features, tooling and shipping have been considered for all the significant STS target system components.

Predicting the lifetime of activated components is the first important operational and planning requirement. Table 5.5 shows the lives and end of life factor for all significant STS activated components. None of the components are predicted to be life-of-facility; therefore, the ability to change components is planned. Where lifetimes are difficult to estimate, a planning figure is provided. 
Table 5.5. Estimated activated component life-times at full power, $5000 \mathrm{hr} / \mathrm{yr}$ operation

\begin{tabular}{|c|c|c|c|c|c|c|}
\hline Component & Material & Limit & Dose & \begin{tabular}{|c|} 
Calculated \\
Design \\
Life
\end{tabular} & $\begin{array}{c}\text { Planned } \\
\text { Design } \\
\text { Life }\end{array}$ & Notes \\
\hline \multicolumn{7}{|c|}{ Maintenance Lifetime Based on dpa Damage } \\
\hline & & dpa & dpa/yr & Years & Years & \\
\hline Target Shroud & $316 \mathrm{~L}$ & 12 & 0.3 & $>20$ & 10 & Actual life is unknown \\
\hline PBWW & Al & 40 & 2.3 & 17.4 & 1.6 to 18 & Depends on criteria \\
\hline Moderator/Reflector & Al & 40 & 7.8 & 5.1 & 2.1 & Cadmium burn-up dependent \\
\hline \multicolumn{7}{|c|}{ Maintenance Lifetime Based on Helium } \\
\hline & & $\begin{array}{c}\text { He Limit } \\
\text { (appm) }\end{array}$ & $\begin{array}{c}\text { Rate } \\
(\text { appm/yr) }\end{array}$ & Years & Years & \\
\hline Proton Beam Window & Al & 2000 & 1335 & 1.5 & 1.6 to 18 & Depends on criteria \\
\hline Moderator Al Shells & Al & 2000 & 116 & 17 & 2.1 & Cadmium burn-up dependent \\
\hline \multicolumn{7}{|c|}{ Maintenance Lifetime Based on Cadmium Burn-up } \\
\hline & & & $\begin{array}{l}\text { Burnup } \\
(\% / y r)\end{array}$ & Years & Years & \\
\hline Side Decoupler & $\mathrm{Cd}$ & & 46 & 2.1 & 2.1 & Depends on Cd thickness \\
\hline \multicolumn{7}{|c|}{ Maintenance Lifetime Based on Other Factors } \\
\hline Halo & & & & & $>10$ & Based on operating experience \\
\hline Harp & & & & & $>5$ & $\begin{array}{c}\text { Actual life is unknown; FTS experience } \\
\text { indicates }>10 \text { years }\end{array}$ \\
\hline Periscope & & & & & 5 & $\begin{array}{l}\text { First mirror surface condition is life limiting } \\
\text { factor }\end{array}$ \\
\hline Monolith Insert life & & & & & $>10$ & Guide up-grades or failures \\
\hline Monolith plugs & & & & & $\sim 1-2 / \mathrm{yr}$ & Changed for new instrument installations \\
\hline
\end{tabular}

Figure 5.77 shows a tooling set-up for removal of a PBW assembly along with a comparable operation at the FTS.

In keeping the philosophy of relying on proven techniques, all the vertically oriented components are designed to be changed using the in-situ disassembly procedure successfully implemented in FTS. This arrangement is based on a six-step process;

- Temporary removal of passive shield blocks above the active component. Shielded containers required for lower blocks held in the high bay during maintenance operations.

- Destructive removal of cooling water, gas and vacuum tubes, and instrumentation sensors into Type A commercial shipping containers.

- Removal of the active module into a custom, heavily shielded handling container designed to be handled inside the target building (Figure 5.77),

- Installation of a new, fully assembled active module (shielding is not required),

- Reinstallation of upper passive shield blocks, and 
- Transfer of spent, activated module into commercial, over-the-road, shipping container in the transfer bay

Note: monolith inserts will be changed using this same procedure employing horizontal tooling.

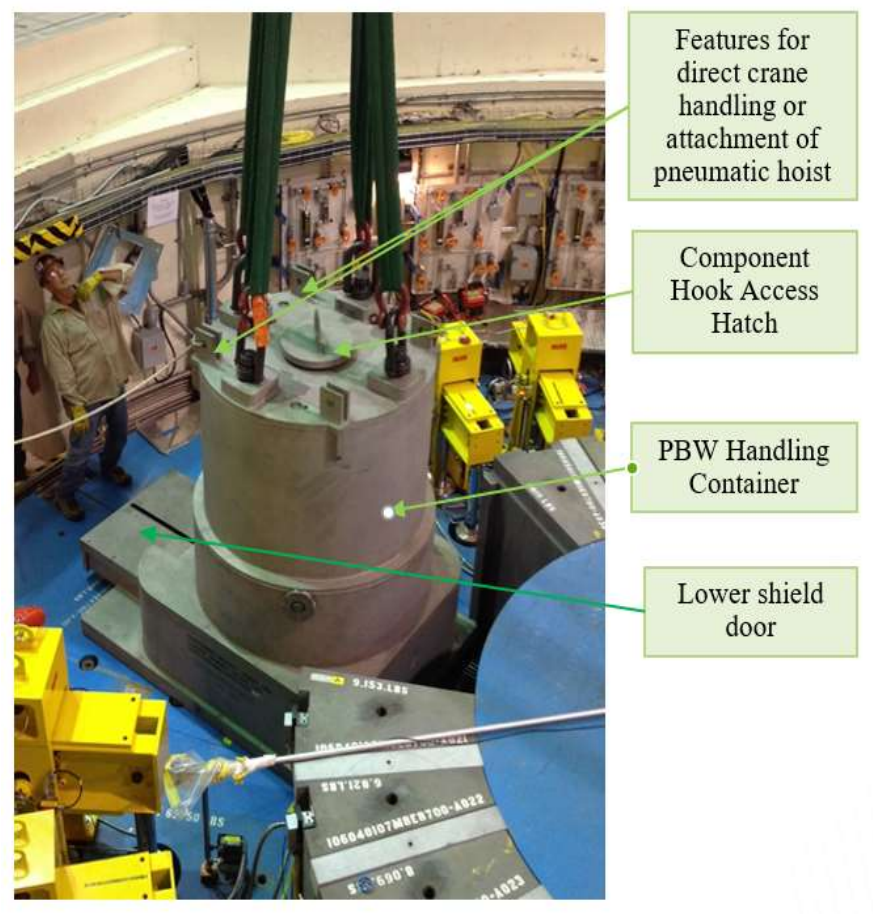

Figure 5.77. PBW window removal operations as proposed for STS and as implemented at FTS.

Apart from the target, all the components will be changed using a procedure similar to the FTS proton beam window change-out pictured in Figure 5.77. Most of these can be handled without disturbing neighboring assemblies; however, the moderator/reflector plug must be moved horizontally on in-vessel rails after removal of the periscope plug to enable it to be removed vertically (see Figure 5.78) 


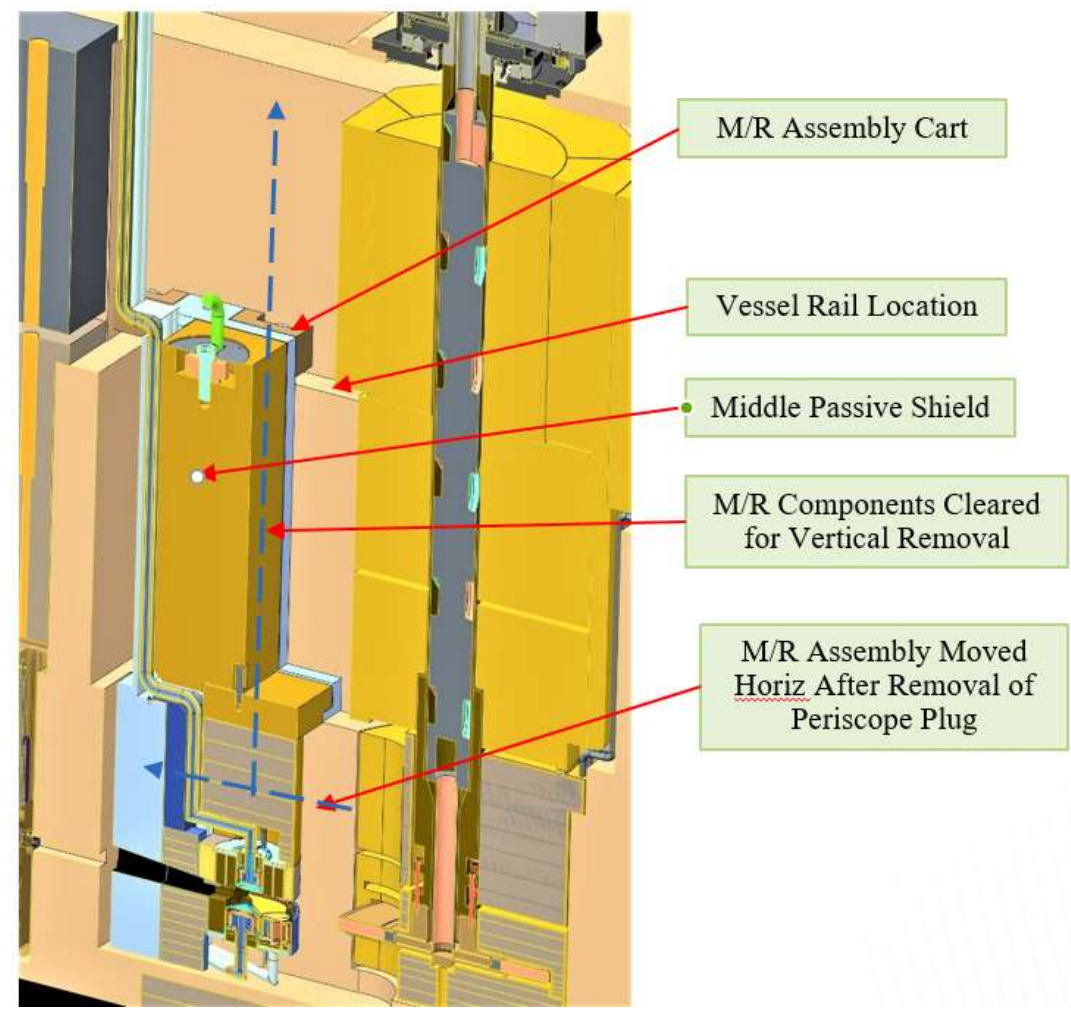

Figure 5.78. The moderator/reflector plug moved horizontally to clear target disk to allow vertical removal.

As noted in Section 5.3.6 there are options in STS operations and mechanical design that can substantially affect the lifetime of the PBW that have not yet been fully explored. Consequently, the life of the assembly, which also includes the Halo monitor, may range from less than 2 years to over 10 years. Regardless of the final determination, the STS design will accommodate tooling and procedures for change out in a one-week period as is currently required for the FTS PBW.

Target change outs are expected to require as much as 3-4 weeks due to four challenging issues:

1) The periscope plug must be removed and the moderator/reflector assembly must be retracted 0.4 meters to provide vertical access to the target disk. This involves the disconnection of numerous water pipes, gas lines and instrumentation sensors. Parting and reconnecting the three cryogenic moderator lines will be particularly difficult and time consuming. Incorporating flexible hoses into the system will be evaluated in a trade-off study of reliability and pipe sizing vs. handling time.

2) A substantial amount of passive shielding above the target must be removed and safely stored in the high bay. The number of operations is not considered to be a time constraint; however, the number and amount of shielding required to protect personnel will be substantial. Handling the cooling water lines for the lower shields will also be complicated by the desire to reuse these pieces, which eliminates the proven cut-and-discard option used on components to be discarded.

3) A significant amount of residual heat ( 2000 to 3000 watts) must be removed from the target disk throughout the handling operations. This will require an active cooling system throughout the handling process. Alternative passive methods will be studied in the next phase of design. 
4) Provisions must be made for removing the axle from the disk while shielding personnel. This will involve the use of a remote, long-handled cutting tool and the addition of some complexity in the handling container to allow for parting the axle inside the shielded cylinder.

Figure 5.79 shows the proposed target handling container with a built-in closed loop cooling system. Additional backup safety systems may be required to address certain failure modes. Removing heat from a disk with a failed shroud has also not been fully addressed except to assume that forced gas ventilation will be required.

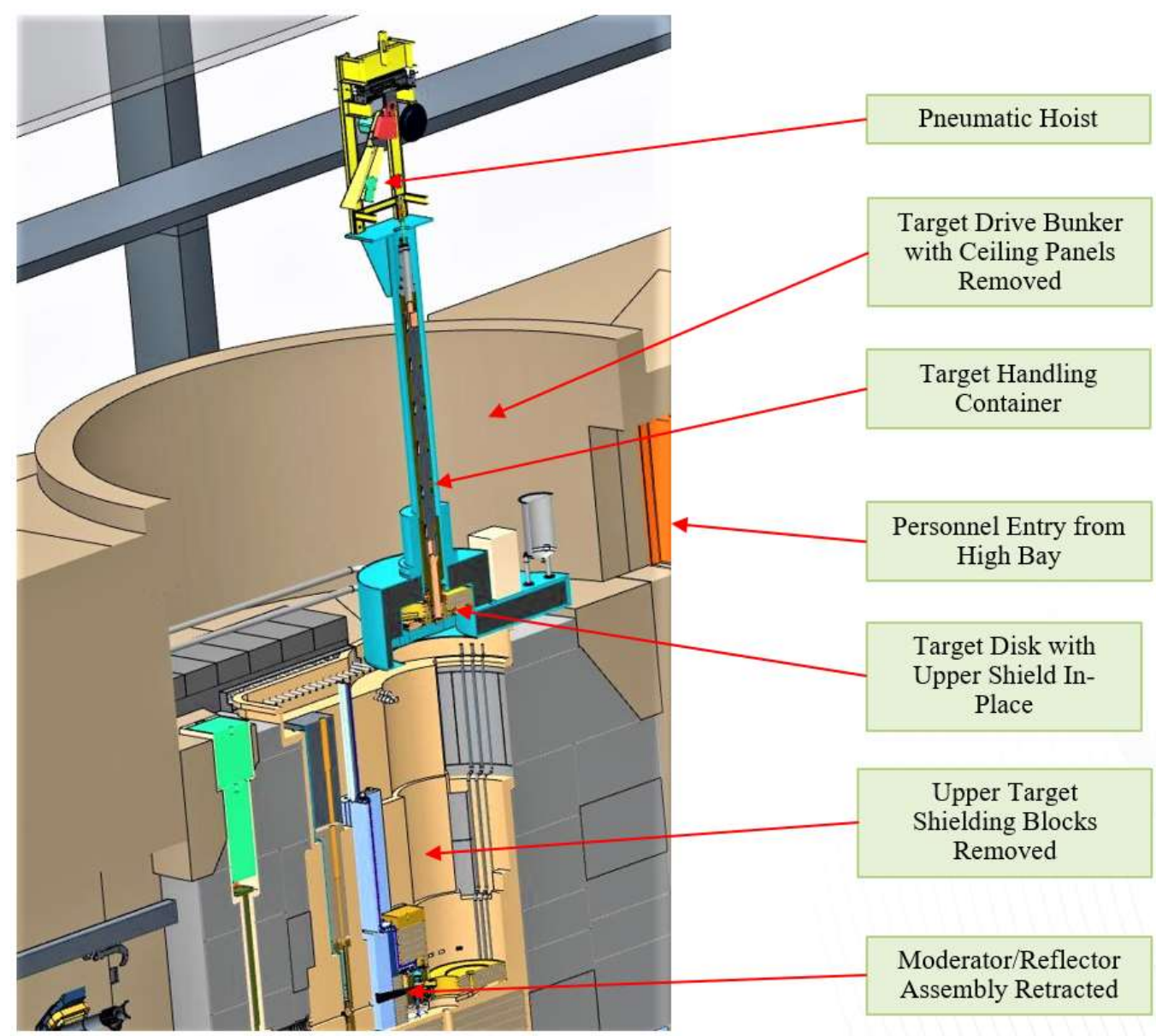

Figure 5.79. Target handling container positioned on core vessel during removal operation.

Monolith inserts will be changed using the same basic procedure used for the vertical assemblies; however, the tooling will be designed to pull spent inserts and push new inserts into position (Figure 5.80). The tooling will also be used to perform the same operation to place inserts inside a commercial shipping container while positioned on the high bay floor. 


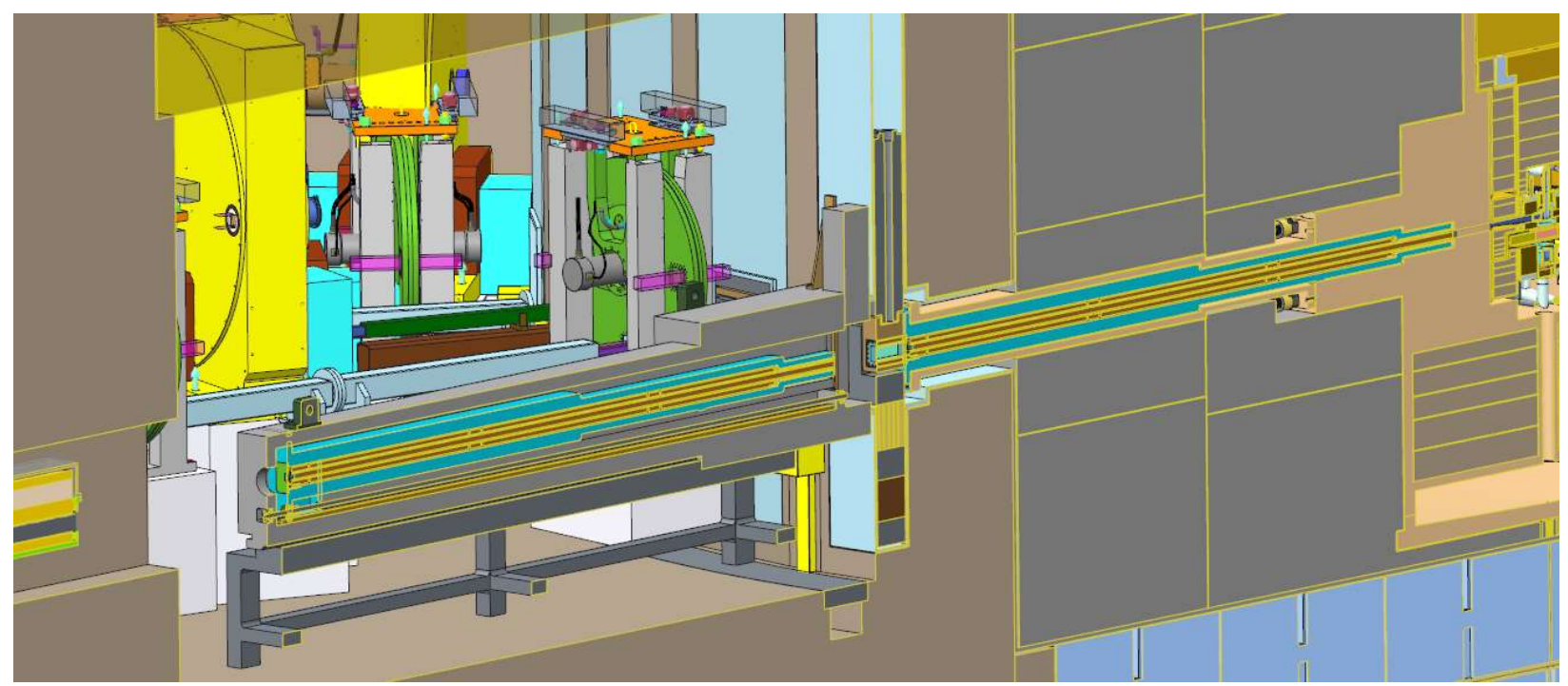

Figure 5.80. Monolith insert handling machine located in the guide bunker.

\subsubsection{Waste Transfer Bay}

Most STS activated waste will be transferred directly to commercial shipping casks in the target building high bay. In this category are typical Type A solid wastes: lightly contaminated or activated components such as pipe sections and upper shield blocks. Highly activated components such as the target disk, moderator/reflector assembly and proton beam window require a transfer bay (Figure 5.81). The primary function of this facility is to enable operators to perform waste transfer operations remotely. Most importantly, the cell enables personnel to remotely install shielding using a small bridge crane and video cameras to enable hands-on cask closure operations.

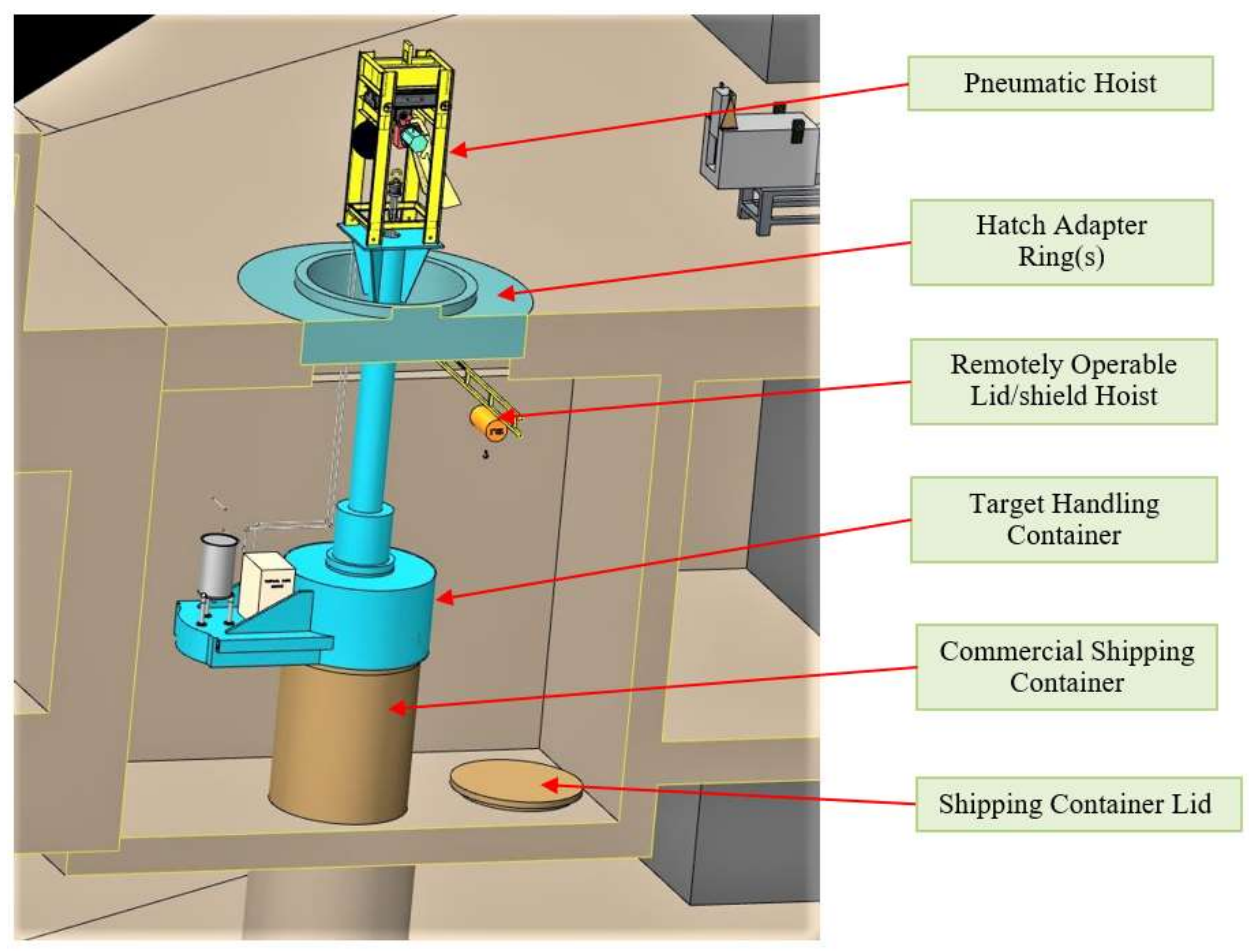

Figure 5.81. Target handling container positioned in transfer bay. 
In order to provide the capability to disassemble and inspect spent targets in the future, additional features are being considered for the transfer bay. These include the installation of liquid drains, ventilation connections and inserts for shielded electrical and instrumentation pass-throughs. If the bay is considered for PIE activities, it will require adequate shielding to accommodate an open target after a year of decay. It is assumed that during this period, tooling designed to address a specific failure will be developed and installed. Given the relatively low activation of the decayed target, the PIE equipment can be based on commercially available tools and machines. For example, the bay will operate with video cameras rather than shielding windows.

\subsubsection{Activated Component Handling and Post Irradiation Examination}

An analysis of known and speculated STS operations that might require a shielded service cell led to a determination that the target building does not need a fully capable cell with substantial personnel shielding and dexterous remote handling. The possibility of converting the transfer bay for use as a PIE facility will be studied further during the next design phase.

The review of possible PIE requirements began with a top-level evaluation of the relevance to STS of the four basic shielded service cell functions performed at current accelerator based neutron facilities.

1) Stationary, horizontal cart mounted targets require cells to enclose both the activated cart and the target during target exchanges. Since the rotating target is more logically handled in bottom loading, shielded handling containers positioned above the monolith this function does not apply to the STS.

2) Facilities with material testing programs require specialized, shielded cells with tooling systems to remove, handle and open highly activated sample containers. More sophisticated cells may include transport packaging and material testing capability. This capability is not required since the STS does not have a material testing or sampling function. Including this in the target station would require the addition of a significant amount of additional hardware in the monolith as well as a shielded handling facility.

3) Handling and packaging of spent components such as proton beam windows modules may be performed in a shielded service cell. STS provides this capability with a dedicated waste transfer bay specifically designed to enable these functions to be performed safety and efficiently as described in Section 5.5.3.

4) Facilities using unproven technologies such as the FTS mercury target require the ability to investigate ongoing performance to address failures and make improvements. This is a potentially desirable reason to have a shielded service cell in STS, therefore; the trade-offs related to this function have been carefully considered for all the STS components; but, especially the target components.

\subsubsection{Failure investigation of target system components other than the rotating target}

A shielded service cell with dexterous manipulator capability is not considered to be required to investigate failures in the non-target components for many reasons. Most importantly, experience has shown that failures can be successfully investigated by non-destructive means, sometimes before being removed from the monolith. For example, pipe failures can be detected by isolating loops and performing a simple pressure check. Video cameras can be used to inspect components during removal operations or with advanced planning while the components are in shielded handling containers. Experience has also shown that the probability of a systematic failure that could justify destructive evaluation is very low 
since all the STS target system components other than the target assembly are based on proven materials, designs and technologies as described throughout the engineering section of this document. Deviating from the use of proven technologies would carry a risk if the potential for future failure investigation is required since the complexity of core components would require specialized, remotely operable tooling to perform an adequate examination. Without prior knowledge or the location or nature of a component failure it would be impractical to have an adequate set of tooling on hand to investigate specific failures in a timely manner. The difficultly of evaluating failures in components is highlighted by the effort required to find faults in un-activated components with direct hands-on access.

STS core component modules, other than the target disk, are relatively small and could be evaluated in the FTS cell if necessary. While this is not a desirable solution it is a viable option that can be reasonably considered.

\subsubsection{Failure investigation of the rotating target drive assembly}

All the active target components are installed in the drive module located in the target drive bunker above the monolith. This arrangement is specifically configured to ensure that all these items, which are assumed to have lives shorter than the target disk, can be accessed hands-on during maintenance outages.

\subsubsection{Failure investigation of the target shroud and axle}

The target shroud and axle will be fabricated as welded assemblies using 316L stainless steel. This material is well understood and has been extensively studied as part of the FTS mercury target development activities. Based on the proven reliability of the FTS water shroud as well as the excellent performance of $316 \mathrm{~L}$ in other nuclear facilities the likelihood of a dpa damage related failure is low. Furthermore, since the material, fabrication and assembly techniques used in these components are well established failures are most likely to be exceptional rather than a systematic failure.

However, the target shroud and axle could experience two types of failures; water leaks or distortion. The water leaks could result from material defects, manufacturing errors (i.e., faulty welds) an axle/disk joint failure or a proton beam excursion. Since all these failures will inherently be external, video inspection and conventional leak check techniques will be particularly effective. For example, nondestructive examination with direct viewing scopes and cameras during change-out or with the in-situ periscope will be effective for locating specific axle and shroud failure locations. A service cell could make the inspection more convenient but the addition of examination features in the target handling container will provide the same capability.

The maximum normal operating temperature of the shroud is less than $60 \mathrm{C}$, well below the $150 \mathrm{C}$ ASME limit for the design stress used to size the shroud. Since the water pressure will be constant with the beam on or off, loading cycles are assumed to be less than 5000 in a 10-year period or once per week consequently fatigue will not be a significant design factor. Furthermore, the assembly welds are intentionally located in relatively low stress zones to avoid efficiency limitations. Therefore, the probability of proton beam related damage to the disk is considered acceptably low.

There are several proton beam failures that could result in a shroud failure including, excessive proton beam pulsing $(>15 \mathrm{~Hz})$, delivery during maintenance and over-focusing. All these cases have been addressed in the hazards analysis study [5] which cites several layers of protection to insure against these, worst case events. These include two credited engineering controls (CEC)to mitigate the loss of cooling events. A Target Protection System (TPS) similar to the one validated in FTS designed to trip the proton beam in the event of a loss of cooling or target rotation. And a CEC to prevent the proton beam from exceeding the design pulse rate; integrated power and innate kicker rep rate limits. 


\subsubsection{Failure investigation of the tantalum-tungsten blocks}

The requirement for a service cell in the STS target building comes down to a trade-off of conservative target block design vs the cost and space penalty of the cell. A comparison of the STS target block operating conditions to the conditions at other facilities illustrates the former point.

- Stress: It is calculated that the STS blocks at approximately the same tantalum and tungsten stresses as the LANSCE target and at about $65 \%$ of those experienced in the ISIS second target station. As noted earlier the design stress level of tungsten is approximately $35 \%$ of the design limit of $200 \mathrm{MPa}$.

- Temperature: Calculated STS block temperature maximums ranging between slightly below those at LANSCE to less than $50 \%$ of the maximum tungsten temperature in the ISIS second target station.

- Cycles: Taking advantage of the rotating configuration the STS target blocks experience cyclical loading at a rate between $1 \%$ and $3 \%$ of currently operating stationary targets.

- Lifetimes: A design lifetime of ten years is predicted for the STS target. Although this is roughly twice the lifetimes experienced at currently operating facilities with fixed targets, the actual loading on the STS target blocks is substantially less given the lower exposure.

- Tantalum Erosion: Erosion of the tantalum clad is a function of temperature and water flow rate. Erosion has not been reported to be a problem at existing facilities but it should be noted that the designed cooling water flow rate inside the STS target disk is $3 \mathrm{~m} / \mathrm{s}$ which is $30 \%$ of the ISIS TS1 flow rate. Additionally, Plansee Ltd states that tantalum is not subject to oxygen or water attack at less than $200 \mathrm{C}$ or 3 times the calculated STS operating temperature.

Although the STS target blocks are conservatively designed there are still several failure modes to be considered; In general, all involve the structural break-down of the tantalum clad alone or in conjunction with a fracturing of the tungsten core. In all cases these failures result in the water erosion of the tungsten and contamination of the return water flow. This in turn results in unacceptable contamination of the process water system and change-out of the target. This is a well-known issue since many tantalum cladtungsten targets have experienced this type of failure; therefore, STS includes full flow filters on the return water line to contain much of the contamination. Containing the contamination allows the leaking target to remain in service until personnel access to the process water systems becomes difficult. The decrease in neutron production would ultimately be detected by instruments through the observation of irregular neutron flux as the failed plate interacts with the proton beam.

Stress induced fracturing of the tungsten inside the tantalum clad would result in higher temperatures to occur in tungsten pieces without conductive contact with the water-cooled tantalum clad. The result of this type of failure is difficult to predict however certain factors are understood. Some of these may be relatively benign; for example, neutron production would not significantly change if the tantalum clad contained the tungsten pieces. Similarly, the stress fracture would tend to relieve the load on the tungsten block and could stabilize the failure, and given the high temperature stability of tungsten (3422 C melting point), a higher operating temperature alone is not likely to be an issue. But, thermal expansion could result in a clad failure which would initiate the water erosion of the tungsten.

Debonding of the tantalum clad in a significant area of the tungsten block could have a thermal impact similar to fracturing of the tungsten. The debonded area will have poor heat transfer characteristics and will therefore cause the tungsten temperature to rise. An analysis of this failure will be included in the next phase of engineering design. 
Proton beam excursions cited in the target shroud failure modes could also cause a target block failure. As noted in the above discussion, the safety credited systems are designed to prevent these events.

Thermal shock waves inside the tungsten have been considered as a possible source of solid target failures. A study performed by J.R.J. Bennett et al. of Rutherford Appleton Laboratory [21] indicates that this should not be an issue for the STS target; however, the subject will receive more analysis in the next phase of design.

Finally, it is recognized that not all failure modes can be foreseen. Consequently, the STS design team continues to consider the possibility of designing the transfer bay for future up-grade to a special purpose PIE facility.

\subsubsection{Service Cell construction and operational considerations}

Without a shielded service cell conceptual design based specific set of functional requirements it is not possible to provide an exact configuration or data. However, some general observations can be made based on previous experience with shielded service cells at ORNL and other facilities. First, it is understood that service cells are in essence expensive and complex facilities. Based on recent work at ORNL in support of the FRIB project along with current conventional facilities estimates for cell construction it is estimated that a functional STS cell would be substantial and highly variable depending on size and complexity. Cost continues to be a factor during operations since service cells require constant monitoring and up-keep.

A highly dexterous STS service cell capable of performing a wide variety of operations would require a substantial amount of space for the cell itself, the operator's gallery, service gallery, load-out room and HEPA ventilation system. The original stationery target cell and attendant facilities required over $460 \mathrm{~m}^{2}$ on two floors. Even if the cell is never fully out-fitted with maintenance tooling the loss of instrument service area inside the target building would be significant loss to the facility. Alternatively, a dedicated operation focused on a specific task could possibly be located in a more compact space.

Based on the relatively simple mercury target PIE in the FTS service cell and the high level of radiation in a newly removed target disk it is estimated that disassembly of a target could require several months assuming all the specialty tooling was in-place. Since development and installation of tooling typically requires over a year, this time could also be used to adapt the transfer bay to accommodate the PIE operation

Completing a trade-off study of the various options available to address target block PIE will be part of the next phase of target station design. The study will include experienced FTS operations personnel, mercury target PIE team members and the STS design team.

\subsubsection{Hazards Analysis}

A formal evaluation of the target station hazards was begun early in the design process with the goal of identifying safety issues that required engineered solutions. The Hazards Analysis process took advantage of the FTS Hazard Analysis Report and Final Safety Assessment Report and the extensive experience of the FTS operations group and the team of SNS engineers who participated in the FTS safety studies. The first informal draft of the Preliminary Hazards Analysis (PHAR), covering 213 events, was issued in October 2016 [3]. The study continues to be up-dated with the first formal PHAR scheduled to be issued in conjunction with the CD1 document package. 
The HA study determined that the principle STS safety concern is limiting public and collocated, on site personnel radiation exposures in accordance with DOE Standard 1189 "Integration of Safety into the Design Process" [22]. In this standard, the 'public' is defined as a person located at the nearest site boundary. A 'collocated worker', is interpreted per ANS Standard 2.26 to mean a person at a distance of $100 \mathrm{~m}$ from a facility (building perimeter) or estimated release point.

The most significant exposure events involved the release of a fraction of the target radiological inventory either because of a hydrogen detonation adjacent to the target or because of tungsten vaporization in steam. Hydrogen detonation is most likely to occur due to seismic events greater than the design basis, which fail hydrogen boundaries within the core vessel and allow air ingress due to other failures. Vaporization events are driven by loss of cooling with continued beam operation. The HA covers all possible loss-of-cooling scenarios due to cooling system failures, target shroud failures or beam failures such as over-focusing which fail the target shroud due to high temperatures. Other events which could fail the target shroud causing a loss of cooling are excessive beam pulsing with $>15 \mathrm{~Hz}$ or a loss of rotation leading to excessive temperatures.

The consequences of a seismic event resulting in a detonation are limited by the passive configuration of the shielding which has a small free volume adjacent to the target on the order of $0.2 \mathrm{~m}^{3}$ or less. Even a stoichiometric hydrogen/air mix in this volume has a very small target mass release fraction. Based on analysis performed for the FTS, the proton beam operation is assumed to be stopped by the seismic event.

Two active credited engineering controls (CEC) are proposed to mitigate the loss of cooling events. A Target Protection System (TPS) similar to the one validated in FTS designed to trip the proton beam in the event of a loss of cooling or target rotation. And a CEC to prevent the proton beam from exceeding the design pulse rate; integrated power and innate kicker rep rate limits.

Because the rotating target has a large surface area to transport heat to the adjacent shielding, if the beam is shut off, the activation decay does not heat the target above the threshold for vaporization (approximately $800{ }^{\circ} \mathrm{C}$ ). Therefore, there is no target vaporization either for seismic events, which fail the cooling system, or for loss of cooling without the beam.

Off-site, boundary doses were calculated using a formula that considered factors such as the amount of activated material that becomes airborne, dose conversion, atmospheric transport using local weather data, breathing intake and cloud shine. The methodology was the same as used for the first target station. Based on the SNS Final Safety Assessment Document (FSAD) [23] dispersion factors, for a ground level release, the on-site dose levels at $100 \mathrm{~m}$ are assumed to be a factor of 20.4 greater than the off-site exposure. This approach was used for very conservative worst case accidents involving a hydrogen detonation next to the tungsten blocks or loss of cooling with target vaporization. All cases resulted in an unmitigated boundary dose $<1 \mathrm{rem}$ and a collocated worker dose $<20 \mathrm{rem}$. These are well within the historically acceptable DOE limits for local office approval.

It is important to note that the proposed STS design does not attempt to prevent a failure of the moderator hydrogen vessels or piping inside the vessel. This is very difficult to do in a seismic event due to the requirement to anchor all neighboring components. And analysis has shown that even the most conservatively imagined hydrogen detonations do not result in unacceptable releases.

\subsubsection{Waste Management}

The operation of the Second Target Station (STS) will generate numerous types of waste from normal operations and maintenance activities. Per U.S. Department of Energy (DOE) Order 435.1, Radioactive Waste Management, and DOE Manual 435.1-1, Radioactive Waste Management Manual, planning shall 
be performed to address the entire life cycle for all waste streams before generation. Proposed STS operations fit within the overarching framework developed to address all waste streams generated by the SNS. All waste streams have been identified and disposal paths have already been established.

\subsubsection{DOE Low-Level Waste (LLW) and Mixed Low-Level Waste (MLLW)}

DOE Headquarters (DOE-HQ) has addressed planning for low-level waste (LLW) and mixed low-level waste (MLLW). The Record of Decision for the Department of Energy's Waste Management Program: Treatment and Disposal of Low-Level Waste and Mixed Low-Level Waste; Amendment of the Record of Decision for the Nevada Test Site was issued February 18, 2000. The decisions contained in this document serve as the programmatic bases for the current master LLW and MLLW disposition maps maintained at DOE-HQ. Four decisions addressing LLW treatment and disposal and MLLW treatment and disposal have been identified. First, DOE decided that each site will perform minimal treatment on its LLW, although each site may perform additional treatment where useful to decrease overall costs. However, it was noted that this decision does not preclude DOE's use of commercial treatment facilities, consistent with DOE regulations and policies. Second, DOE decided to establish regional LLW disposal at two DOE sites: the Hanford Site and the Nevada Test Site (NTS). In addition to disposing of their own LLW, the Hanford Site and NTS will receive and dispose of LLW that is generated and shipped (by either truck or rail) by other sites that meets the waste acceptance criteria. Although the decision acknowledged that DOE will continue, to the extent practicable, on-site disposal at the Oak Ridge Reservation (ORR), current disposition maps show the majority of ORR-generated LLW going to NTS. Third, DOE decided to conduct regional MLLW treatment at numerous sites, inclusive of ORR, as would be consistent with current site treatment plans. This decision does not preclude DOE's use of commercial treatment facilities, consistent with DOE regulations and policies. Fourth, DOE decided to establish regional MLLW disposal operations at two DOE sites: the Hanford site and the NTS. In addition to managing their own MLLW, the Hanford site and NTS will receive and dispose of MLLW generated and shipped (by truck or rail) by other sites, consistent with permit conditions and other applicable requirements. Once again, this decision does not preclude DOE's use of commercial disposal facilities, consistent with current DOE regulations and policies.

Per DOE O 435.1, "Radioactive waste produced as a result of operations of DOE accelerators is lowlevel waste ...". At present, the SNS sends LLW to both the Nevada National Security Site (NNSS), formerly known as the Nevada Test Site (NTS), and to commercial facilities. The SNS sends MLLW to commercial treatment and/or disposal facilities. The LLW and MLLW generated by the STS will be managed in the same manner.

\subsubsection{DOE Hazardous Waste}

Hazardous waste planning is also addressed by DOE-HQ. However, the treatment and/or disposal of hazardous waste is well understood, and numerous commercial treatment and/or disposal options are available. Therefore, in the Record of Decision for the Department of Energy's Waste Management Program: Treatment of Non-wastewater Hazardous Waste, issued July 30, 1998, DOE states its decision to continue use of off-site facilities for the treatment of major portions of the non-wastewater hazardous waste generated at DOE sites. Concurrently, DOE recognized that the ORR may treat some of its own non-wastewater hazardous waste on-site, where capacity is available in existing facilities and where treatment is economically favorable. All hazardous waste generated at the SNS are sent to commercial facilities for treatment and/or disposal. Hazardous waste generated by the STS will be managed in the same manner. 


\subsubsection{DOE Sanitary and Industrial Waste Handling and Disposal}

Sanitary/industrial waste is typically not included in DOE-HQ planning initiatives because treatment and/or disposal of sanitary/industrial waste is well understood and occurs at all DOE facilities. Typically, the treatment and/or disposal of sanitary/industrial waste is regulated by state programs administered by the state of the respective DOE facility. Sanitary/industrial waste generated on the ORR consists of recyclable materials, such as metals, and non-recyclable materials such as glass, paper, and wood. Nonrecyclable materials are disposed in state-permitted land disposal units (sanitary/industrial landfills).

\subsubsection{Pollution Prevention and Waste Reduction}

Regardless of waste categorization, design, construction, and operation of the STS will aggressively implement pollution prevention principles to prevent and/or reduce the amount of waste to be generated. As delineated in the Pollution Prevention Act of 1990, the STS shall employ the following four principles with respect to all operations: 1) pollution should be prevented and reduced at the source whenever feasible; 2) pollution that cannot be prevented should be recycled in an environmentally safe manner whenever feasible; 3) pollution that cannot be prevented or recycled should be treated in an environmentally safe manner whenever feasible; and 4) disposal or other release to the environment should be employed only as a last resort and should be conducted in an environmentally safe manner. Furthermore, the STS embraces the ORNL Pollution Prevention Policy whereby "we are committed to providing a safe and healthful working environment for all staff, guest scientists and engineers, and visitors; protecting the general public and the environment from unacceptable ES\&H risks; operating in a manner that protects and restores the environment; and integrating pollution prevention into planning and decision-making." As such, STS will follow ORNL Pollution Prevention guidelines.

\subsection{VALUE ENGINEERING}

The beginning of a major project such as STS is the most effective time to incorporate value engineering principles. With this in mind, the STS team has kept cost, efficiency, and value at the center of all design decisions. Without an earlier design concept to compare to, it is not possible to provide cost savings; however, value engineering results can be identified in the design process as well as the design itself. The design team used all available paths to implement value engineering in the design. These include the use of existing SNS facilities and procedures, use of proven component designs, trade-off studies, and hazards studies and validation testing.

\subsubsection{Use of Existing Facilities and Procedures}

Significant design and operating cost savings have been realized through the shared use of existing SNS resources and knowledge. Many STS systems are purposely configured to mirror their FTS counterparts. These include the process water loops, cryogenic systems, sample environment support, waste management and instrument operations. In all these cases STS will follow the same procedures, equipment configuration, operations and vendor support contracts. Of particular importance is remote handling, where many of the tools and procedures currently in use in the FTS will be used or copied in STS. The design of remote tooling also benefits from direct input from experienced FTS operators. These include complex long handled tools, hook extenders and the purpose built pneumatic hoist. This benefit has been maximized by designing all the basic STS activated component handling activities to match the proven FTS procedures.

Based on FTS experience with the existing SNS facility, STS undertook a space programming study [24] in cooperation with an outside AE contractor with an extensive background in the design of science facilities. The study reviewed all functions associated with FTS and STS and made a preliminary space 
allocation based on the future scientific and operational requirements of both facilities (see Figure 5.82). The scope of the study included eleven primary STS facilities including

- $\quad$ R2T2 Tunnel \& Service Building

- $\quad$ Target Building II

- Long Guide Enclosures

- Transition Building

- $40 \mathrm{M}$ Instrument Building

- Long Instrument Building

- Zeeman's Building

- Central Laboratory and Office Building II

- Central Utility Building II

- Site Utilities.

A key result of this study was a $25 \%$ reduction in gross square footage of the total STS project area defined in the STS Technical Design Report [25]. In addition, there are ongoing efforts to further reduce the STS footprint through better integration with existing FTS facilities as the design advances.

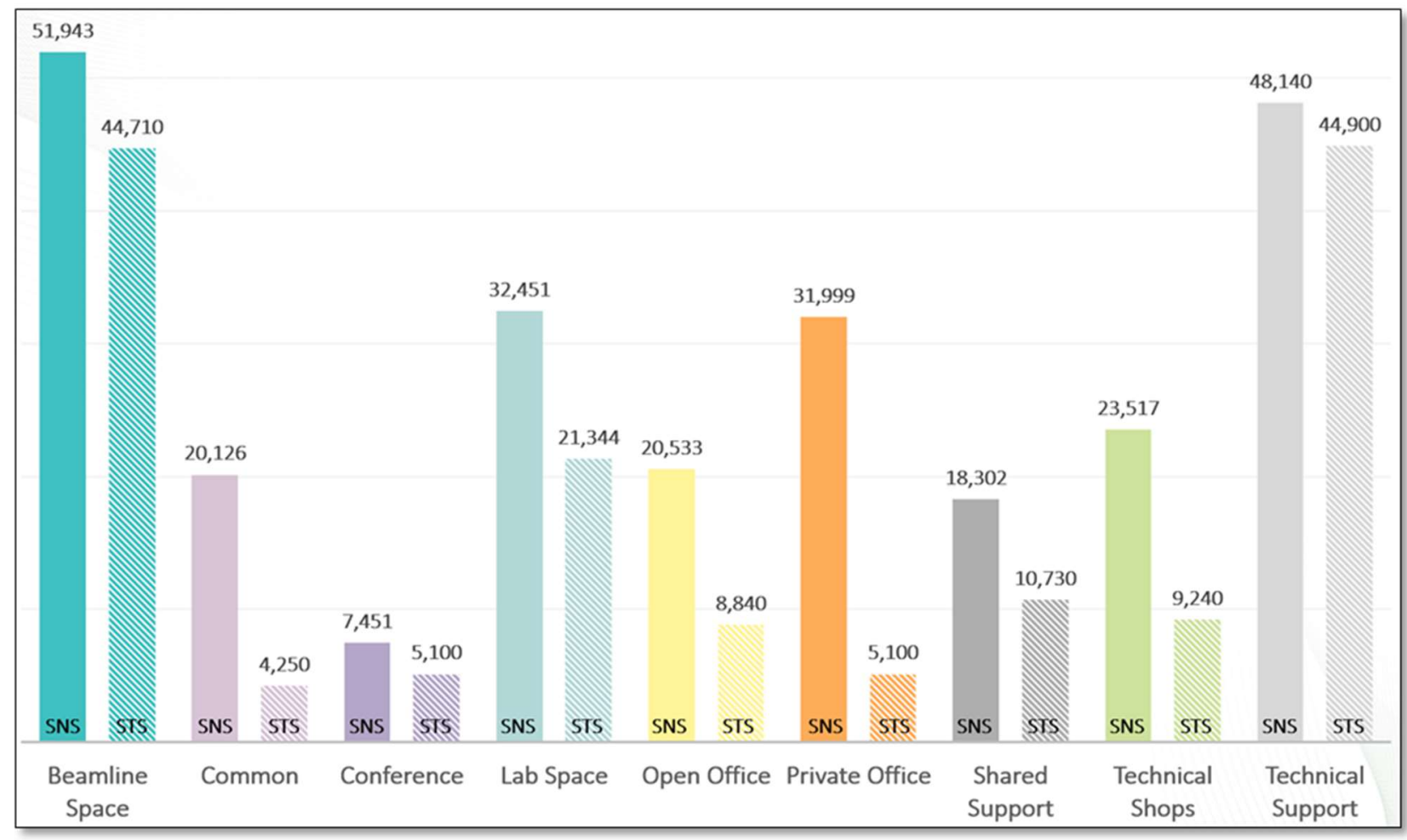

Figure 5.82. Comparison of FTS and STS facility space utilization requirements.

STS has addressed the entire life cycle for all waste streams per U.S. Department of Energy (DOE) Order 435.1, Radioactive Waste Management, and DOE Manual 435.1-1, Radioactive Waste Management Manual. Based on SNS and ORNL experience, STS operations will fit within the overarching framework developed to address all waste streams generated by the facility, and all waste streams have identified paths to disposal including handling procedures and tooling concepts. 


\subsubsection{Visits and Discussions with Other Facilities}

STS has attempted to take full advantage of the experience of other accelerator based neutron facilities. In fall 2016, the STS management team visited PSI and ISIS with the explicit goal of learning how to improve and simplify the layout of the STS facility to reduce cost and/or enhance experimental operations. Component design, operations and maintenance activities were also covered during these visits.

Manufacturing of tantalum clad tungsten blocks is recognized as possibly being the most challenging aspect of the target station design. Consequently, the STS engineers have reached out to the experienced ISIS and LANSCE target engineers for detailed advice regarding all aspects of the fabrication process. This outreach effort saved considerable development time and provided the basis for the current target block development program.

\subsubsection{Minimum Component Development}

Historically, implementation of new, innovative technologies has proven to be a costly element in new facilities. STS plans to advance the state-of-the-art in neutron instrument technologies, but within target systems, a management decision to adapt component designs based on existing, proven technologies has resulted in efficient, low risk components such as the harp, halo, proton beam window, cryogenic systems, utilities systems, vessel, bulk shielding and moderators. Even most components in the rotating target are based on solid targets used at other facilities.

Reusing proven designs benefits the project in many ways beyond simplifying engineering. This approach also allows STS to take advantage of known vendors, reliable cost estimates and realistic fabrication schedules.

\subsubsection{Design Optimization}

Attention to basic design and value engineering principles resulted in several significant areas in which the design of the target systems have been simplified and improved. Elimination of a service cell is a result of the decision to use proven technologies weighed against cost and risk. As reviewed in Section 5.5.4, aside from the waste transfer function accommodated in a purpose-built bay, a service cell could not be justified. The resulting savings in capital cost, operating cost and functional building space are substantial.

Operating experience at FTS was used to reduce the size and cost of the maintenance shutters in STS. Rather than the 2-meter-long slab shutters embedded in the FTS monolith iron shield, the 0.25-meter-long STS shutters are both substantially smaller, less costly and easier to maintain. The cost savings are extended to a reduction in the complexity and cost of the monolith iron shield and utilities distribution system.

Because the FTS instruments were staged over several years, they tended to be designed as separate, stand-alone systems with minimal consideration of using standard designs for various beam line optical, structural, or shielding components. Consequently, there are multiple versions of neutron guide housings and vacuum enclosures, different methods of constructing beam line shielding and removable shield blocks, and distinct designs of many optical components like secondary shutters, beam benders, and optical slits. While not necessarily being efficient, this approach provides STS designs that were optimized through several iterations. One area in which FTS was successful in implementing standard designs was choppers. A centralized group developed a common design approach which led to 
interchangeable spare parts among many of the beam lines and significant operational efficiencies. Some custom choppers were developed where needed, but in most instances the standard designs were used.

While it is understood that neutron beam lines have different needs and will always require a degree of customization, it is a goal within STS to incorporate common designs where practical, to learn from the experiences gained in the FTS beam line designs, and to incorporate best practices from FTS and other neutron scattering facilities. This will become more apparent as the instrument designs begin to evolve and the requirements of multiple instruments are better understood so that shared component designs can accommodate the needs of different instruments

\subsubsection{Trade-off Studies}

In conjunction with the STS Programming Study [24], a second planning document was developed, the STS Elevation Study [5]. Its objectives were to "coordinate the experimental and conventional facilities design with respect to five key factors that will influence the functionality, cost and overall site design of the project. These factors, which are the subject of this Study, include overall project planning, site and facility access, long instrument guide enclosure design, R2T2 tunnel design and sub-grade waterproofing/drainage." Major recommendations from this study included, among others,

- Constructing the R2T2 Tunnel in a flat ( $0 \%$ slope) configuration so that the proton beam entered the STS at the same elevation as in the FTS

- Reconfiguring the Target Building II to include a third floor level

- Inclusion of a Transition Building which provides long neutron beamline maintenance access and user access between the sample environment workshop and the long instruments

- Separating the long instruments into individual buildings to be constructed as needed rather than as a consolidated building

- Employing the Trench Guide Enclosures to link the long instrument buildings with the Target Building II

\subsubsection{Early Hazard Analysis}

Developing a detailed hazard analysis study early in the project insured that the proposed STS design will not require costly future changes to be compliant with DOE guidelines. The study led to the discovery of the significant safety issue associated with the loss-of-coolant accident of a fixed solid target and also resulted in a better understanding of the relatively benign nature of the rotating target configuration. Consequently, the system benefitted from the introduction of new, less costly and less complex systems such as the rotating target, elimination of the moderator helium boundary and a reduced seismic requirement for the target building structure. By incorporating such major design changes early in the STS conceptual development, significant costs savings will be realized as compared to making such changes later in the design process.

\subsubsection{Design Validation Mockups and Prototypes}

Early validation of new target system concepts with full scale, proof-of-principle, mockups and prototypes has been an efficient means of confirming basic concepts and enabling the engineering team to consider design improvements. The most obvious example is the rotating target prototype, which has successfully demonstrated many fabrication and operating features of the proposed system. This assembly 
also pointed the designers towards the shorter and less complex drive module now proposed. Likewise, the periscope mockup has proved that it is possible to view the target area with a simple, flat mirror arrangement instead of costly, radiation-hardened fiber optic systems. It also showed that the optics tubes do not have to be as elaborate as the computational model indicated. Ultimately, the target block fabrication prototypes now in process will likely prove to be the most important validation test. If successful this program will develop an efficient, commercial source for production of the actual target.

Additional design validation prototypes will be considered as development needs are identified. Potential candidates for validation mockups include the monolith insert installation and alignment scheme and the proposed method of implementing real-time alignment of beam line imaging optics.

\subsubsection{Vendor Consultations}

Where new technologies or unique fabrication issues are considered, the STS design team has reached out to knowledgeable vendors to confirm concepts and learn the state-of-the-art. The most important examples are the several design evaluation discussions with the two-leading neutron beam guide manufacturers regarding the proposed STS implementations of monolith inserts, imaging optics alignment, and standardization of beam guide vacuum housings. Input received in these discussions has been included in the current design, and plans are to maintain contact with these vendors as STS beam guide designs progress.

The SNS chopper team has developed and maintained relationships with their counterparts at other neutron scattering facilities; consequently, the team understands the current state of the art designs and technologies as well as those being considered for next-generation choppers. In addition, SNS also maintains regular contact with chopper and magnetic bearing manufacturers to keep them apprised of the proposed STS chopper sizes and performance requirements to ensure STS needs fall within their current and planned capabilities, or where they don't, so that STS understands where it will need to invest development funds to support its neutron chopper needs. These relationships also allow SNS to potentially reduce STS chopper design costs through collaborations with other neutron facilities, if timing allows.

In general, fabrication of the proposed components is well understood since the designs are based on existing components. However, the rotating target has a few new and unique features that are being designed in conjunction with leading fabrication vendors. This includes the double helix shielding plug in the center of the axle and the water channeling features in the shroud. 


\subsection{REFERENCES}

[1] A Second Target Station for the Spallation Neutron Source, SNS 100000000-TR0029-R00, Oak Ridge National Laboratory, Oak Ridge, Tennessee, Oct. 2007.

[2] F. Gallmeier, Moderator Studies for a SNS Short-Pulse Second Target Station, STS03-31-TR0004-R00, Oak Ridge National Laboratory, Oak Ridge, Tennessee, May 2013.

[3] Spallation Neutron Source, Second Target Station Facility Hazard Identification and Evaluation-FY16 Draft; ORNL Document No. STS03-31-ES0001, R00; September, 2016.

[4] HAZARD ANALYSIS FOR THE SPALLATION NEUTRON SOURCE TARGET SYSTEM (U), SNS Document No. 102030102-ES0002-R00, Dec 1999.

[5] Spallation Neutron Source - Second Target Station, STS Elevation Study, SNS Document No. STS05-51TD0002, R00, October 20, 2016.

[6] Michael Baumgartner, et.al., Review of ORNL Second Target Station Target Design Alternatives, September $22-23,2015$.

[7] "The ESS Helium Cooled Rotating Target," Consorcio ESS-BILBAO \& Instituto de Fusion Nuclear \& ESS-ERIC, A. Aguilar, et.al., 13th IWSMT, Oct. 30th - Nov. 4th, 2016.

[8] "CSNS target development," Jia Xuejun, The International Workshop on Pulsed Spallation Neutron Sources, J-PARC, JAEA, Tokai, Japan, Nov 9-13, 2009.

[9] Jones, Leslie; STFC Rutherford Appleton Laboratory, email correspondence; 15 March 2017.

[10] Jones, Leslie; STFC Rutherford Appleton Laboratory, email correspondence; 15 March 2017.

[11] "ISIS Target Analysis", Dan Wilcox - High Power Targets Group, SNS Engineering Visit 22/05/2015

[12] “Thermo-Mechanical Analysis of ISIS TS2 Spallation Target,” Dan Wilcox; High Power Targets Group, Rutherford Appleton Laboratory, 5th High Power Targetry Workshop, Fermilab, 21/05/2014.

[13] "Fabrication of a tantalum-clad tungsten target for KENS," Masayoshi Kawai, et. al., Journal of Nuclear Materials 296 (2001), 312-320.

[14] A.T. Nelson, et.al., Fabrication of a tantalum-clad tungsten target for LANSCE, Journal of Nuclear Materials, RNL/TM 2015/24, January 2015.

[15] Personal communication via a telecom with Jeremy Moor at ISIS February 15

[16] "Design and Testing of a Prototype Spallation Neutron Source Rotating Target Assembly," MJ Rennich, et al., ICANS XIX, 19th meeting on Collaboration of Advanced Neutron Sources, Grindelwald, Switzerland, March $8-12,2010$.

[17] K. Farrell, Journal of Nuclear Materials 97 (1981) 33-43.

[18] K. Farrell, Materials Selection for the HFIR Cold Neutron Source, ORNL Technical Report ORNL/TM-99208 (1999).

[19] "Nested Mirrors for X-rays and Neutrons," G. Ice, R. Barabash, A. Khounsary, web.ornl.gov/sci/physical_sciences_directorate/mst/x-ray/pdf/pubs/2009/Ice_Nest09.pdf

[20] "Precision Magnet Movers for the Final Focus Test Beam," G. Bowden et al., SLAC-PUB-95-6132, June 1995.

[21] J.R.J. Bennett, et.al., "Thermal shock measurement and modelling for solid high-power targets at high temperatures' Journal of Nuclear Materials' 377 (2008) 285-289.

[22] DOE Standard 1189 "Integration of Safety into the Design Process".

[23] Spallation Neutron Source Final Safety Assessment Document for Proton Facilities, 102030103- ES0018R00, Spallation Neutron Source, Oak Ridge, TN, June 2005.

[24] Spallation Neutron Source - Second Target Station, STS Programming Study, SNS Document No. STS0551-TD0001, R00, October 20, 2016.

[25] Second Target Station Technical Design Report, John Galambos et al., ORNL/TM 2015/24, January 2015. 
APPENDIX A. STS PLANNING INSTRUMENT SUITE 


\section{APPENDIX A. STS PLANNING INSTRUMENT SUITE}

A number of instrument concepts were developed in response to a series of workshops that explored the impact and need for neutrons in the next decade and beyond [1]. These concepts were used as a planning suite of instruments for an initial site layout and moderator selection and optimization. This list forms the basis of Table A.1 that gives a brief description of the instruments. Instruments marked with * were identified by an October, 2015 workshop as high priority and most have been the subject of more thorough consideration as described in Section 3 [2]. The table has been updated since the workshop to reflect workshop recommendations.

Table A.1. Instrument concept descriptions

\begin{tabular}{|c|c|}
\hline Name & Description \\
\hline \multicolumn{2}{|c|}{ Diffractometers } \\
\hline EWALD - Enhanced Wide Angle Laue Diffractometer & Optimized for small macromolecular single crystals \\
\hline HighResPD - High Resolution Powder Diffractometer & $\begin{array}{l}\text { Optimized for highest resolution studies of powder } \\
\text { samples }\end{array}$ \\
\hline NeSCry - Neutron Single Crystal Diffractometer & $\begin{array}{l}\text { Optimized for small single crystals with high low-Q } \\
\text { resolution, and an emphasis on magnetic structure }\end{array}$ \\
\hline VERDI - Versatile Diffractometer & $\begin{array}{l}\text { Optimized for magnetic structure studies of both powder } \\
\text { and single crystals }\end{array}$ \\
\hline $\begin{array}{l}\text { MENUS - Materials Engineering by NeUtron } \\
\text { Scattering }\end{array}$ & $\begin{array}{l}\text { Optimized for diffraction studies of engineering } \\
\text { materials }\end{array}$ \\
\hline \multicolumn{2}{|c|}{ Spectrometers } \\
\hline BWAVES - Broad-range Wide Angle Velocity Selector & $\begin{array}{l}\text { Indirect geometry spectrometer with high energy } \\
\text { resolution and a very broad dynamic range of energy } \\
\text { transfers (uses chopper for final energy selection) }\end{array}$ \\
\hline CHESS - Chopper Spectrometer for Small Samples & $\begin{array}{l}\text { Cold neutron chopper spectrometer optimized for very } \\
\text { small samples }\end{array}$ \\
\hline $\begin{array}{l}\text { HERTZ - High Energy Resolution Terahertz } \\
\text { Spectrometer }\end{array}$ & $\begin{array}{l}\text { Cold neutron chopper spectrometer optimized for } \\
\text { standard/large samples and relatively high energy } \\
\text { resolution }\end{array}$ \\
\hline $\begin{array}{l}\text { JANUS - Inelastic scattering instrument (INS) } \\
\text { Instrument for Catalysis }\end{array}$ & $\begin{array}{l}\text { Hybrid indirect/direct geometry spectrometer optimized } \\
\text { for irreversible phenomena and in situ sample } \\
\text { manipulation }\end{array}$ \\
\hline MBARS - Mica Backscattering Spectrometer & $\begin{array}{l}\text { Indirect geometry spectrometer optimized for ultra-high } \\
\text { energy resolution studies (quasielastic neutron } \\
\text { scattering) }\end{array}$ \\
\hline $\begin{array}{l}\text { SPHIINXS - Spherical Indirect Inelastic Crystal } \\
\text { Spectrometer }\end{array}$ & $\begin{array}{l}\text { Indirect geometry spectrometer optimized for broad- } \\
\text { band inelastic measurements of small samples }\end{array}$ \\
\hline $\begin{array}{l}\text { XTREME-X - Extreme Environment Multi-Energy } \\
\text { Spectrometer with Crystal Analyzers }\end{array}$ & $\begin{array}{l}\text { Indirect geometry spectrometer optimized for } \\
\text { measurements restricted to the horizontal scattering } \\
\text { plane by extreme sample environments }\end{array}$ \\
\hline \multicolumn{2}{|c|}{ Multi-Modal Instruments } \\
\hline $\begin{array}{l}\text { HiResSWANS - High Resolution Small/Wide Angle } \\
\text { Neutron Scattering }\end{array}$ & $\begin{array}{l}\text { A combined small angle neutron scattering } \\
\text { (SANS)/diffractometer optimized for structural studies } \\
\text { from molecular to tens of nanometers }\end{array}$ \\
\hline
\end{tabular}




\begin{tabular}{cl}
\hline Name & \multicolumn{1}{c}{ Description } \\
\hline ZEEMANS - High Magnetic Field Beam Line & $\begin{array}{l}\text { Versatile instrument that integrates neutron } \\
\text { spectroscopy, diffraction, reflectometry and SANS with } \\
\text { a very high field magnet }\end{array}$ \\
\hline
\end{tabular}

Table A.1. Instrument concept descriptions (continued)

\begin{tabular}{ll}
\hline \multicolumn{1}{c}{ Name } & \multicolumn{1}{c}{ Description } \\
\hline SANS-1 & \multicolumn{1}{c}{ Large Scale Structures } \\
SANS-2 & $\begin{array}{l}\text { General purpose SANS } \\
\text { SANS with TBD specialization } \\
\text { Combines measurements of specular reflectivity, off- } \\
\text { WASABI - Wide And Small Angles with Big Intensity } \\
\text { specular scattering and grazing incidence neutron } \\
\text { scattering for measuring 3D structures in films } \\
\text { OIKR - Quite Intense Kinetics Reflectometer }\end{array}$ \\
& $\begin{array}{l}\text { Optimized for rapid specular reflectivity measurements } \\
\text { fingle instrument setting }\end{array}$ \\
& $\begin{array}{l}\text { Horizontal geometry reflectometer optimized to deliver a } \\
\text { variable beam profile onto a sample surface as small as 1 } \\
\text { mm² }\end{array}$ \\
\hline
\end{tabular}

\section{REFERENCES}

[1] Instruments for Emerging Science: A Science Case for the Second Target Station, ed. K. W. Herwig and D. A. Tennant, Neutron Sciences Directorate, ORNL, 2014 (unpublished).

[2] Second Target Station Workshop, Co-chairs: M. R. Eskildsen (University of Notre Dame) and B. Khaykovich (Massachusetts Institute of Technology), https://public.ornl.gov/conferences/neutrons/STS2015/index.shtml. 